\title{
Interpretation of Sun-Induced Chlorophyll Fluorescence for Remote Sensing of Photosynthesis
}

\author{
Peiqi Yang
}




\section{PhD graduation committee}

Chair and Secretary

Prof.dr.ir. A. Veldkamp Supervisor

Prof.dr.ing. W. Verhoef Co-supervisor

Dr.ir. C. van der Tol

University of Twente

University of Twente

University of Twente

Members

Prof.dr. A. Damm

Prof.dr. Z. Su

Prof.dr. R. Zurita Milla

University of Zurich

University of Twente

University of Twente

Prof.dr. P.R.J. North

Swansea University

Prof.dr. C. Simmer

University of Bonn

ITC dissertation number 326

ITC, P.O. Box 217, 7500 AA Enschede, The Netherlands

ISBN: $\quad 978-90-365-4591-4$

DOI: $\quad 10.3990 / 1.9789036545914$

Printed by: ITC Printing Department

(c) Peiqi Yang, Enschede, The Netherlands

(c) Cover design by Job Duim and Benno Masselink

All rights reserved. No part of this publication may be reproduced without the prior written permission of the author.

DT FACULTY OF GEO-INFORMATION SCIENCE AND EARTH OBSERVATION 


\title{
INTERPRETATION OF SUN-INDUCED CHLOROPHYLL FLUORESCENCE FOR REMOTE SENSING OF PHOTOSYNTHESIS
}

\author{
DISSERTATION
}

to obtain

the degree of doctor at the University of Twente, on the authority of the rector magnificus, prof.dr. T.T.M. Palstra on account of the decision of the graduation committee, to be publicly defended on Thursday, July 19, 2018 at 12.45

by

Peiqi Yang

born on December 16, 1989

in Jiangxi, China 
This dissertation is approved by:

Prof.dr.ing. W. Verhoef (supervisor)

Dr.ir. C. van der Tol (co-supervisor) 
Look further into things and be comfortable with them. 



\section{Acknowledgments}

Completing my $\mathrm{PhD}$ research has been a challenge but also a beautiful journey full of unique experience. It would not have been possible without the support and guidance of many people. They have made the challenges into amazing experiences. I would like to express my sincere gratitude to all of them.

I consider myself very fortune to have had the chance to pursue $\mathrm{PhD}$ with my promoter, Prof. Wout Verhoef, who has been a truly dedicated mentor. The foundations of Wout's work are both powerful and elegant. I could not have maintained interest in my work without being profoundly impressed by the elegance and clarity of his work. Special gratitude goes to Wout. Thank you for guiding me toward the challenging and intriguing research. You are so generous in sharing your knowledge and experience. Your constructive comments, notes and very smart suggestions have always been a spark for considering new ways to improve work and further rationalize my choices.

I am deeply grateful to my supervisor Dr. Christiaan van der Tol, who first of all gave me the opportunity to do this $\mathrm{PhD}$ in ITC, and then has been closely observing my progress, continuously providing me with very efficient scientific guidance. My $\mathrm{PhD}$ has been an amazing experience and I thank Christiaan wholeheartedly, not only for his tremendous academic support, but also for giving me so many wonderful opportunities. The joy and enthusiasm he has for his research was contagious and motivational for me. I could not have imagined having a better advisor and mentor for my 
PhD study.

Tracing the origins of my academic interests, I have to review my MSc study. In my first course at Beijing Normal University, 'Optical Remote Sensing of Vegetation', each student was asked to give a short introduction of one classical model, including PROSPECT, LIBERTY, Suits, SAIL and Kuusk's model. The neat equations written by Wout somehow attracted me and the SAIL paper in 1984 was the challenge I choose for myself. The precise derivation in his work relieved the emotional resistance of a surveying engineer to the uncertainties of remote sensing and evoked my great interest. One year later, Prof. Zhigang Liu sent me an archive of the SCOPE model and asked me to organize a brainstorm in group meeting. I was so impressed by the capability of the model and rigorous thought of the developers. I must thank Prof. Liu for guiding me in the way of science. Suggested by him, I joined the 4th workshop of fluorescence in Paris and met Wout and Christiaan there.

Big thanks also go to the colleagues in ITC. Thank all the office mates Nastia, Cesar, Junping and Egor for encouragements during the long journey and sharing fun in daily life. Many thanks to Prof. Bob Su for the enlightenments, insightful discussion and support during my PhD. Big thanks to WRS colleagues for their support and company during these years. Special thanks to Anke and Tina for helping me with many aspects in my stays, and to Loes for being kind and supportive to me. I would also like to thank Benno and Job for the assistance in making nice posters and the efforts in designing the thesis cover. Many thanks go to my friends and colleagues, Sylo, Bagher, Behnaz, Myriam, Jan, Harm-Jan, Georgios, Sammy, Megan, Gabriel, Lichun, Murat, Novi, Sox and Yasser for cultivating ITC a happier workplace.

Discussion and exchange of ideas on the contents of my work also took place with many people outside of ITC. I thank my colleagues in the FLEX and OPTIMISE community for the fruitful exchange of ideas with a special mention to Prof. Alex Damm, Prof. Uwe Rascher, Prof. Anatoly Gitelson and Dr. Joanna Joiner. I would like to express my sincere thanks for their 
kind advices, comments, suggestions and inspiring ideas. It was fantastic to have the opportunity to work with them. Thanks also go to MaPi, Dr. Anke Schicking and Dr. Onno Muller for generously hosting me in Acchen, to Marco for the joyful discussion about research, culture, music and food, to Sheng and Chao for the company in Cyprus and Sofia.

Many thanks to my Chinese community in ITC for their company and help. Special thanks to the Chinese-Twekkelerveld neighbors, Xiaolong, Junping, Xu, Shaoning, Zhihui, Linlin, Hong, Wen, Qiang, Chengliang, Ruosha, Lianyu, for the delicious food and joyful parties in the four years. Special thanks also go to Dr. Tiejun Wang, Dr. Yijian Zeng, Dr. Xuelong Chen, Dr. Donghai Zheng, Dr. Binbin Wang, Xiaoling, Fangyuan, Xi, Ying, Yiwen, Yifei, Yifang, Pei, Mengna, Haili, Xin, Mengmeng, Fashuai and Zhenchao. You have made this place my second home.

A very special gratitude goes out to China Scholarship Council for helping and providing the funding for the work, and to OPTIMISE and ITC for supporting my $\mathrm{PhD}$ research.

This dissertation would not have been possible without the consistent encouragements and support from my families. I feel most deeply indebted to my parents for their cultivation and education, to my sister, brothers, sistersin-law and brother-in-law for keeping me away from family obligations I should fulfill, to my parents-in-law for the endless support and understanding, and to my dear nieces and nephews for bringing joy to the whole family. My deepest gratitude goes to my beloved wife Jing for her endless support, love, care and understanding. You were always there to advise and encourage me and to share my worries and happiness. Thank you.

\section{Peiqi Yang}

June 2018

Enschede, The Netherlands 


\section{Contents}

Contents vi

1 Introduction 1

1 Why monitoring plant photosynthesis? . . . . . . . . . 2

2 How to monitor plant photosynthesis? . . . . . . . . . . 3

3 Sun-induced chlorophyll fluorescence for photosynthesis . . . 7

4 Challenges in photosynthesis monitoring from SIF . . . . . . 13

5 Objectives and organization of the thesis . . . . . . . . 15

2 Interpreting SIF measurements by using radiative transfer $\begin{array}{ll}\text { models } & 17\end{array}$

1 Introduction . . . . . . . . . . . . . . . 19

2 Materials and methods . . . . . . . . . . . . . . 21

3 Results..................... 34

4 Discussion ...................... 44

5 Conclusion ........................ 49

3 Linking canopy scattering of SIF with reflectance 51

1 Introduction . . . . . . . . . . . . . . . 53

2 Theoretical basis .................. 56

3 SCOPE simulation method .............. 64

4 Simulation results . . . . . . . . . . . . 68

5 Discussion ..................... 74

6 Conclusion ..................... 82 
4 Canopy structure effects on SIF $\quad 83$

1 Introduction . . . . . . . . . . . . . . . . . 85

2 Theoretical basis . . . . . . . . . . . . . . . 89

3 Evaluation of fPAR model and FCVI . . . . . . . . . . . . . . 93

4 Results . . . . . . . . . . . . . . . . . . 101

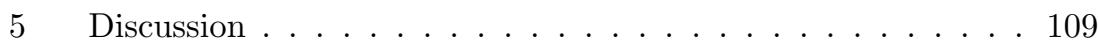

6 Conclusions . . . . . . . . . . . . . . . . . 113

5 Radiative transfer in multi-layer canopies 115

1 Introduction . . . . . . . . . . . . . . 117

2 Description of mSCOPE . . . . . . . . . . . . 119

3 Materials and Methods . . . . . . . . . . . . . . 132

4 Results .................... 136

5 Discussion .................... 140

6 Conclusion ...................... 143

$7 \quad$ Appendices ...................... 143

6 Concluding remarks and prospects $\quad 147$

1 Conclusions and implications . . . . . . . . . . . . 148

2 Further challenges on the way ahead . . . . . . . . . . 150

$\begin{array}{ll}\text { Bibliography } & 153\end{array}$

$\begin{array}{llr}7 & \text { Summary } & 179\end{array}$

$\begin{array}{lll}8 & \text { Samenvatting } & 183\end{array}$

$9 \begin{array}{ll}9 u \text { thor's biography and publications } & 187\end{array}$ 



\section{List of Figures}

1.1 The emission of chlorophyll fluorescence of a leaf (Davidson et al., 2003) and a typical fluorescence spectrum. . . . . . . . . 7

2.1 Workflow of interpretation of HyPlant reflectance and SIF data by using SCOPE. . . . . . . . . . . . . . . . . 22

2.2 Overview of the study area and the flight plan before (June 30th, day 1) and during (July 2nd, day 2) the heat wave. The crops investigated in the study and three reference panels are marked with polygons. The background image was acquired on 24th August 2016 (from Google Earth). . . . . . . . . . . . . . . . . 23

2.3 Representative radiance measurements of vegetation from the DUAL (black) and the FLUO (red) module, respectively. . . . . 24

2.4 Bare soil reflectance on day 1 and on day 2. The buffers represent variation (i.e. standard deviation) in the selected pixels. . . . . . 30

2.5 RGB, temperature, SIF at $687 \mathrm{~nm} F_{687}$ and at $760 \mathrm{~nm} F_{760}$ images of the experiment area before and during the heat wave. Crops are marked in the RGB image: 1: rapeseed; 2: corn; 3: barley; 4: wheat. ..................... . . . . . . 35

2.6 Red fluorescence $\left(F_{687}\right)$ and far red fluorescence $\left(F_{760}\right)$ on day 1 (before the heat wave) and day 2 (during the heat wave). The horizontal and vertical error bars represent the standard deviation of measurements at day 1 and day 2 , respectively. . . . . . . . 37 
2.7 Reflectance measurements before (day 1) and during (day 2) the heat wave in the spectral region of $400-2500 \mathrm{~nm}$ and of 400 $-700 \mathrm{~nm}$. The buffers represent the standard deviation of the measurements. . . . . . . . . . . . . . . . . 37

2.8 Normalized difference vegetation index (NDVI) and photochemical reflectance index (PRI) before (day 1) and during (day 2) the heat wave. The horizontal and vertical error bars represent the standard deviation of measurements at day 1 and day 2, respectively. 38

2.9 Upper panels: Measured (blue) and modelled (black) reflectance spectra of the four crops before the heat wave (day 1). Lower panels: the residual after spectral fitting (line) (i.e., the difference between measured and simulated apparent reflectance ), and the standard deviation of the measurements (grey area). . . . . . . . 39

2.10 Upper panels: Measured (black) and modelled (blue) reflectance spectra of the four crops during the heat wave (day 2). Lower panels: the residual after spectral fitting (line) (i.e., the difference between measured and simulated apparent reflectance), and the standard deviation of the measurements (grey area). . . . . . . .

2.11 Upper panels: canopy structural contribution to TOC SIF (radiative transfer factor of SIF, $\Gamma_{r t}$ ) estimated from SCOPE before (day 1) and during (day 2) the heat wave of the four crops. Middle panels: the difference of $\Gamma_{r t}$ before and during the heat wave $\left(\Delta \Gamma_{r t}\right)$. Lower panels: the uncertainty of $\Gamma_{r t}\left(\sigma_{\Gamma}\right)$ caused by the uncertainty in the reflectance measurements. The buffers represent the standard deviations of the 16 or 8 patches. . . . . .

2.12 Retrieved values of fluorescence emission efficiency (i.e. photosynthetically determinative factor of the TOC SIF) at $687 \mathrm{~nm}$ $\left(\epsilon_{F 687}\right)$ and $760 \mathrm{~nm}\left(\epsilon_{F 760}\right)$ before and during the heat wave of the four crops. The horizontal and vertical error bars represent the standard deviation of measurements at day 1 and day 2, respectively. 41 
2.13 Relative contribution of canopy structure, plant physiology and incident light intensity to the changes in TOC SIF measurements ( $F_{687}$ and $\left.F_{760}\right)$ before and during the heat wave. . . . . . . . . 43

2.14 The efficiencies of three pathways of absorbed energy in photosystems changing with leaf temperature from 15 to $40{ }^{\circ} \mathrm{C}$ : fluorescence emission $\epsilon_{F}$, photochemistry $\epsilon_{P}$, and heat dissipation $\epsilon_{H} . \ldots$

3.1 The interaction between incident light and canopy. The ellipses represent leaves in the canopy, and red edges indicate the leaves are illuminated directly by the light from the top of the canopy. The fluorescence flux and scattered flux are represented by the red and black curve. The plus and minus signs indicate backward and forward side of a leaf, respectively. . . . . . . . . . . . . 58

3.2 Flux and vegetation canopy interaction diagram. The rectangles refer to scattering or fluorescence emission events. The circles refer to fluxes generated from the events. The arrows indicate changes of fluxes. $E$ refers to the incident light. $i_{0}$ is the canopy interceptance, and thus $i_{0} E$ is the light interacting with the canopy. $i_{0} E$ will first be involved in scattering and emission event (s1 and e1), resulting scattered flux and SIF flux, respectively. Part of the fluxes will be observed fluxes by sensor $\left(L_{o}\right.$ and $\left.L_{o}^{F}\right)$. The resulted scattered flux and SIF flux will be involved in scattering and emission events again (s2, s3, e2, e3), and further contribute to the observed fluxes. . . . . . . . . . . . . . . .

3.3 The canopy scattering of $\operatorname{SIF}\left(\sigma_{F C}\right)$ in the spectral region from $640 \mathrm{~nm}$ to $850 \mathrm{~nm}$ simulated by using SCOPE. In the simulations, LAI was set to $0.5,1,2,3$ or 6 . Leaf chlorophyll $C_{a b}=40 \mu \mathrm{g}$ $\mathrm{cm}^{-2}$. Leaf structure parameter $N=1.5$. Leaf mass $C_{d m}=0.01$ $\mathrm{g} \mathrm{cm}^{-2}$. Equivalent water thickness $C_{w}=0.015 \mathrm{~cm}$. LIDFa $=$ -0.5. Sun zenith angle $\theta_{s}=30^{\circ}$ 
3.4 The canopy scattering of SIF $\left(\sigma_{F C}\right)$ in the spectral region from $640 \mathrm{~nm}$ to $850 \mathrm{~nm}$ simulated by using SCOPE. In the simulations, Leaf chlorophyll $C_{a b}$ was set to $5,10,20,40$ or $80 \mu \mathrm{g} \mathrm{cm}^{-2}$. LAI $=3$. Leaf structure parameter $N=1.5$. Leaf mass $C_{d m}=0.01 \mathrm{~g}$ $\mathrm{cm}^{-2}$. Equivalent water thickness $C_{w}=0.015 \mathrm{~cm}$. LIDFa $=-0.5$. Sun zenith angle $\theta_{s}=30^{\circ} . \ldots \ldots \ldots \ldots \ldots$

3.5 SIF emission at $687 \mathrm{~nm}$ and $760 \mathrm{~nm}$ from all the interactions, the first order interactions and multiple interactions changing with LAI. The error bars represent the total range of variation of the 360 scenarios with the same LAI but different leaf properties, leaf orientations or sun zenith angles. Note: SIF from the first order interactions refers to the fluorescence emission by incident light directly. SIF from the multiple interactions is the fluorescence emission excited by scattered light. . . . . . . . . . . . . . . 70

3.6 Radiance of top-of-canopy (TOC) SIF observed at $687 \mathrm{~nm}$ and $760 \mathrm{~nm}$ from all the interactions, the first order interactions and multiple interactions changing with LAI. . . . . . . . . . 70

3.7 Canopy scattering of SIF at $687 \mathrm{~nm}$ and $760 \mathrm{~nm}$ from all the interactions, the first order interactions and multiple interactions changing with LAI. . . . . . . . . . . . . . . . . . . .

3.8 The comparison of partitioning of scattered radiation $(\rho / \tau)$ and partitioning of emitted SIF $\left(\rho_{f} / \tau_{f}\right)$ over the two sides of leaves at $687 \mathrm{~nm}$ and $760 \mathrm{~nm}$ simulated with Fluspect. Simulations with the same leaf structure parameter $(N)$ are marked with the same colour. . . . . . . . . . . . . . . . . . . . . . . .

3.9 The correlation between canopy scattering of SIF $\left(\sigma_{F C}\right)$ and $\frac{R}{i_{0} \omega}$ at $687 \mathrm{~nm}$ and $760 \mathrm{~nm}$ for the group-1 (synthetic leaves) scenarios. Each point represents one scenario. Every leaf in the group had equal reflectance and transmittance $(\rho=\tau=\omega / 2)$, and equal backward and forward fluorescence emission. Note: $R$ is canopy reflectance, $i_{0}$ is the canopy interceptance and $\omega$ is the leaf albedo. 73 
3.10 The correlation between canopy scattering of SIF $\left(\sigma_{F C}\right)$ and $\frac{R}{i_{0} \omega}$ at $687 \mathrm{~nm}$ and $760 \mathrm{~nm}$ for the group-2 (Fluspect leaves) scenarios. Each point represents one scenario. Simulations of scenarios that have the same leaf are highlighted with the same colour. Three individual leaves are respectively marked as red, blue and green for illustration, while the remaining 57 leaves are marked as gray. Note: $R$ is canopy reflectance, $i_{0}$ is the canopy interceptance and $\omega$ is the leaf albedo.

3.11 View zenith angle (VZA) effects on reflectance and SIF at $687 \mathrm{~nm}$ and $760 \mathrm{~nm}$ simulated with SCOPE. Negative values of the VZA represent the backward direction, and positive values represent the forward direction. The key model parameters were set as follows: sun zenith angle $\theta_{s}=30^{\circ}$, relative azimuth angle between sun and view $\Psi=0^{\circ}$, chlorophyll content $C_{a b}=40 \mu \mathrm{g} \mathrm{cm}^{-2}$, leaf structure parameters $N=1.5$, LAI $=3$, leaf inclination parameters LIDFa $=0.5$ and $\mathrm{LIDFb}=0.5 \ldots \ldots \ldots \ldots \ldots \ldots$

4.1 Fluorescence emission efficiency used as input of the SCOPE model in the spectral region from $640 \mathrm{~nm}$ to $850 \mathrm{~nm}$. The markers indicate locations of $740 \mathrm{~nm}$ and $760 \mathrm{~nm} . \ldots \ldots$. . . . . .

4.2 Comparison between NDVI and fPAR, and between $i_{0} \frac{R_{n i r}-R_{v i s}}{R_{n i r}}$ and fPAR for the 10800 scenarios with real soil and non-reflecting soil. The colours represent the number of scenario in a 0.01 by 0.01 grid. . . . . . . . . . . . . . . . . . . . 102

4.3 The radiative transfer factor of fluorescence $\left(\Gamma_{r t}=\mathrm{fPAR} \times \sigma_{F}\right)$ in the spectral region from $640 \mathrm{~nm}$ to $850 \mathrm{~nm}$ simulated by using SCOPE. The dashed lines are the values of FCVI for the five scenarios bounded underneath by non-reflecting surface. . . . . . 103 
4.4 Spider plot for sensitivity analysis of the radiative transfer factor of SIF $\left(\Gamma_{r t}\right)$ at $760 \mathrm{~nm}$. The base values were set as follows. $\mathrm{LAI}=3$. Leaf chlorophyll $C_{a b}=40 \mu \mathrm{g} \mathrm{cm}^{-2}$. Leaf structure parameter $N=1.5$. Leaf mass $C_{d m}=0.01 \mathrm{~g} \mathrm{~cm}^{-2}$. Equivalent water thickness $C_{w}=0.015 \mathrm{~cm}, \mathrm{LIDFa}=0$. Sun zenith angle $\theta_{s}$

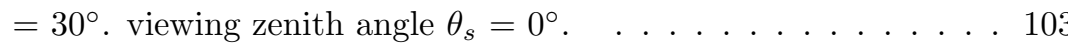

4.5 Comparison between SCOPE simulated fluorescence correcting vegetation index (FCVI) with the radiative transfer factor of fluorescence $\left(\Gamma_{r t}\right)$ at $760 \mathrm{~nm}$ and $740 \mathrm{~nm}$ for the 10800 scenarios with real soil and non-reflecting soil. The colours represent the number of scenario in a 0.005 (FCVI) by $0.005\left(\Gamma_{r t}\right)$ grid. . . . . 104

4.6 Globe maps of NDVI (a), GOME-2 SIF at $740 \mathrm{~nm}$ (b), fluorescence emission efficiency $\epsilon_{F}$ at $740 \mathrm{~nm}$ (c) and PAR (d) in June 2014. . 107

4.7 Comparison between NDVI with SIF and $\epsilon_{F}$ in June in 2014. The colours represent the number of measurements of which the $x$ and $y$ values in a small grid. . . . . . . . . . . . . . . . 108

4.8 Correlations between monthly mean values gridded at 0.5 degrees by 0.5 degrees resolution of SIF, NDVI, $\epsilon_{F}$ and PAR computed for 10 years of global measurements (2007-2016). The arrows indicate the drivers (e.g. PAR is one of the drivers of SIF) . . . . . . . 108

4.9 Light response of the fluorescence emission efficiency at $740 \mathrm{~nm}$ from GOME-2 SIF data (A) and from Van der Tol et al. (2014) model (B). The light responses of FCVI, $\pi$ SIF/PAR retrieved from MODIS reflectance data and GOME-2 SIF data from 2007 to 2016 are presented as well (A). FVCI is shown in the inset in the upper right corner and $\pi \mathrm{SIF} / \mathrm{PAR}$ is shown with dashed grey line. The grey area in (A) indicates the relative probability distribution function (PDF) of the pixels at various light intensities. 109

5.1 Canopy reflectance observations on DOY 173, 206 and 259 in the growing season and soil reflectance (DOY, day of year). . . . . 133 
5.2 Vertical profile of leaf chlorophyll content $\left(C_{a b}\right)$ and LAI in the field datasets acquired on three days in the corn growing season. (Note, $y$ axis represents leaf position. The collar or ear leaf was labelled as leaf 0 . The leaves above or below leaf 0 were identified with a ' + ' or '-' sign, respectively, with the corresponding position number. For example, the first leaf above the collar or ear leaf was identified as +1 , the first leaf below the collar or ear leaf was identified as -1; DOY, day of year).

5.3 Simulation results for the six synthetic scenarios from mSCOPE. a), b) nadir reflectance spectra; c), d) nadir fluorescence spectra (Note, $\mathrm{S} 0$ is a homogeneous scenario, S1-S5 have different vertical distribution of chlorophyll content $\left(C_{a b}\right)$ and leaf water content $\left.\left(C_{w}\right)\right)$. Reflectance spectra from 500 to $650 \mathrm{~nm}$ were enlarged in the grey boxes. . . . . . . . . . . . . . . 136

5.4 Simulation results of the corn canopy on three days in the growing season. upper panel: comparison among measured, mSCOPE modelled, and SCOPE modelled reflectance; lower panel: comparison between mSCOPE modelled and SCOPE modelled fluorescence (Note: the vertical profile of $C_{a b}$ for each canopy was simplified as 3, 7, and 11 layers and implemented in mSCOPE. DOY, day of year). . . . . . . . . . . . . . . . . . . 139 



\section{List of Tables}

2.1 The meteorological conditions during the airborne campaigns before and during the heat wave. . . . . . . . . . . . 25

2.2 The ranges and initial values of the key parameters used in SCOPE . 27

2.3 Temperature of the canopies $\left(T_{c}\right)$ on day 1 and on day 2 , and difference in canopy and air temperature $\left(T_{c}-T_{a}\right) \ldots \ldots 36$

2.4 The retrieved values of soil moisture $\left(S M_{p}\right)$ leaf chlorophyll content $\left(C_{a b}\right)$, leaf water content $\left(C_{w}\right)$, canopy LAI and canopy average leaf angle (ALA) before and during the heat wave. . . . 40

2.5 Relative changes (\%) in TOC SIF measurements at $687 \mathrm{~nm}$ and $760 \mathrm{~nm}\left(F_{687}\right.$ and $\left.F_{760}\right)$, in fluorescence emission efficiency $\left(\epsilon_{F}\right)$ retrieved from $F_{687}$ and $F_{760}$, in radiative transfer factor of SIF $\left(\Gamma_{r t}\right)$ at these two wavelengths before and during the heat wave for the crops. The sign '-' indicates a decrease on day 2 (during the heat wave).

3.1 Summary of SCOPE inputs applied for the generation of the database ....................... 66

4.1 Summary of SCOPE inputs applied for the generation of the database ....................... . . . . . . .

4.2 Parameters used in the analytical estimation of bottom of atmo-

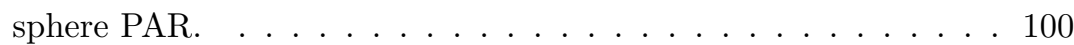

5.1 Main input parameters of SCOPE . . . . . . . . . . . . . . 119 
5.2 Extra input parameters of mSCOPE compared with SCOPE . . 119

5.3 Input parameters of vertical leaf chlorophyll $\left(C_{a b}, \mu \mathrm{g} \mathrm{cm}{ }^{-2}\right)$ and equivalent water thickness $\left(C_{w}, \mathrm{~cm}\right)$ profile in six two-layer canopy scenarios. . . . . . . . . . . . . . . . . . . . . . 132

5.4 Photosynthetically active radiation absorbed (aPAR), net photosynthesis $(A)$ and light use efficiency (LUE) simulated from mSCOPE of the six synthetic scenarios. . . . . . . . . . . 137

5.5 The parameters of canopy structure and leaf properties of the corn canopy retrieved from the TOC reflectance measurements. . 138

5.6 Net photosynthesis simulated from mSCOPE and SCOPE of the corn canopy on the three days. . . . . . . . . . . . . . . . . 139 


\section{List of Abbreviations}

3FLD

APAR

AVHRR

ALA

BOA

BPLUT

BSM

ChlF

DART

DOY

DASF

ESA

FLEX

FLD

fPAR

FOV

FCVI

GPP

GOSAT

GOME-2
Three-bands based FLD

Absorbed Photosynthetically Active Radiation

Advanced Very High Resolution Radiometer

Average Leaf Angle

Bottom-Of-Atmosphere

Biome Parameter Look-Up Table

Brightness-Shape-Moisture

Chlorophyll Fluorescence

Discrete Anisotropic Radiative Transfer

Day Of Year

Directional Area Scattering Factor

European Sapce Agnecy

FLuorescence EXplorer

Fraunhofer Line Depth

Fraction of Photosynthetically Active Radiation

Field Of View

Fluorescence Correction Vegetation Index

Gross Primary Production

Greenhouse gases Observing SATellite

Global Ozone Monitoring Experiement-2 satellite 


\begin{tabular}{ll} 
GSV & Global Soil Vector \\
iFLD & Improved FLD \\
LUE & Light Use Efficiency \\
LAI & Leaf Area Index \\
LIDF & Leaf Inclination Distribution Function \\
LUT & Look-Up Table \\
MODIS & Moderate Resolution Imaging Spectroradiometer \\
NDVI & Normalized Difference Vegetation Index \\
NPQ & Non-Photochemical Quenching \\
NASA & National Aeronautics and Space Administration \\
NO & Numerical Optimization \\
NIR & Near-InfraRed \\
OCO & Orbiting Carbon Observatory satellite \\
PAR & Photosynthetically Active Radiation \\
PQ & Photochemical Quenching \\
PAM & Pulse Amplitude Modulated \\
PRI & Photochemical Reflectance Index \\
PDF & Probalility Distribution Function \\
RTM & Radiative Transfer Model \\
RVI & RatioVegetation Index \\
SIF & Sun-Induced chlorophyll Fluorescence \\
SFM & Spectral Fitting Methods \\
SAIL & Scattering by Arbitrarily Inclined Leaves \\
SCOPE & Soil-Canopy-Observation of Photosynthesis and Energy \\
TOA & Toil-Vetation-ATmosphere \\
\hline
\end{tabular}


TOA Top-Of-Atmosphere

VI Vegetation Index

VIS Visible

VZA View Zenith Angle 



\section{Introduction}


The subject of this thesis is the interpretation of sun-induced chlorophyll fluorescence (SIF) that can be applied to monitoring vegetation photosynthesis from remote sensing techniques. In this introduction first a brief review of remote sensing of SIF for photosynthesis is given. Next, challenges in using SIF are reviewed. The motivation and objectives for writing this thesis are summarized next and this chapter is concluded with a structural overview of the remaining chapters.

\section{Why monitoring plant photosynthesis?}

Plants play a crucial role in our Earth system. They have shaped the environment throughout history and turned our planet into a habitable place (Berry, 2012). The astonishing diversity of plants profoundly molds Earth's climate, the evolutionary trajectory of life and our society.

Photosynthesis is one of the most fundamental processes on Earth and makes plants essential to our world. Photosynthesis happens inside chloroplasts that contain chlorophyll. Many chemical reactions in photosynthesis can be summarized by the classic chemical reaction equation: $\mathrm{CO}_{2}$ (Carbon dioxide) $+\mathrm{H}_{2} \mathrm{O}$ (Water) $\stackrel{\text { Light }}{\longrightarrow} \mathrm{C}_{6} \mathrm{H}_{12} \mathrm{O}_{6}$ (Sugar) $+\mathrm{O}_{2}$ (Oxygen) The reaction converts light energy, water and carbon dioxide into energy-rich organic compounds and oxygen fueling the organisms' activities. The photosynthesis process consumes carbon dioxide (a significant greenhouse gas) from the atmosphere and emits oxygen as a byproduct to the atmosphere making a habitable planet for all living creatures.

Monitoring photosynthesis facilitates a better understanding of what limits photosynthesis and to develop technologies for increasing photosynthetic rate in crops for sustainable yield. Producing enough food to meet the growing demand is a distinct challenge. In the past century, crop yields have kept up with population growth under the effects of the "Green Revolution" which contains the application of knowledge in plant breeding and genetic manipulation (Hawkesford et al., 2013). However, these effects of the "Green Revolution" are fading while the global population is growing (Godfray et al., 2010). This raises concerns about future food security. Modern precision 
agriculture is one way to ensure production. The essence of precision agriculture is real-time monitoring and management of crops, in particular photosynthesis. Moreover, the knowledge on optimizing photosynthesis is crucial for enhancing crop yields. Crop yields have been increased throughout history but there was no or little change in the rate of photosynthesis per unit leaf area (Foyer et al., 2017). By measuring photosynthesis under various environmental conditions, the optimal conditions for photosynthesis per leaf can be determined for various crops.

The measurement of plant photosynthesis also plays an important role in understanding ecological systems and climate changes. Tracking the responses of photosynthesis to climatological variables at several scales will help us to understand the interaction between plants and environment (Grace et al., 2007), for example, effects of atmospheric $\mathrm{CO}_{2}$ enrichment on plant growth (Idso et al., 1987; Ainsworth and Long, 2005; De Graaff et al., 2006), and effects of plants on removal of $\mathrm{CO}_{2}$ (Farquhar et al., 1993). We will have a better understanding of the response of natural ecosystems to the climate change (e.g., rising atmospheric $\mathrm{CO}_{2}$ and global warming) and predict the evolution of plants and ecosystems on Earth (Drake et al., 1997; Bazzaz, 1990).

\section{How to monitor plant photosynthesis?}

The conceptual basis for photosynthesis monitoring is to measure the resources or products involved in this process. More specifically, tracking gas exchange, water-related processes, light flows or organic compounds are the ways to monitor photosynthetic activity and further compute gross primary production (GPP) and net primary production (NPP) at various temporal and spatial scales.

The first global map of photosynthesis was made by using an annual measure of actual evapotranspiration (AET) (i.e. water loss) (Lieth, 1975) in two simple steps. First, the annual AET was estimated from global temperature maps extrapolated from the measurements from around a thousand weather stations in the world. Second, a simple relationship between AET 
and annual NPP was found and applied (Running et al., 2004).

$$
\mathrm{NPP}=3000 \times\{1-\exp [-0.0009695(\mathrm{AET}-20)]\}
$$

The measure of photosynthesis from AET is a rough estimation and it is affected by many other processes on the surface. Gas exchange measurements provide a direct measure of photosynthetic carbon assimilation, and this method is nowadays preferred. Photosynthesis results in the exchange of $\mathrm{CO}_{2}$ (gas) exchange between the atmosphere and plant, and the net rate at which the gases are produced and consumed forms the basis of gas exchange methods for measuring photosynthesis.

Two methods, chamber-based measurements and eddy covariance, are employed to measure the $\mathrm{CO}_{2}$ exchange. In the chamber-based method, a leaf or plant is enclosed in a transparent chamber, and the rate at which the $\mathrm{CO}_{2}$ concentration changes in the chamber is monitored (Long et al., 1996). The chamber can either be sealed without resupply with fresh air or be provided with a constant flow of air, which refer to closed and open systems, respectively. A series of commercial, portable, gas exchange systems are available in the market (e.g. LI6400, LiCor Inc., USA; GFS-3000, Walz, Germany). Results from the use of chambers the methods are mostly limited to the levels of individual leaves or plants. Alternative chamberless methods, particularly those involving eddy covariance (EC), are available and provide an essential method for assessing the gas exchange of whole communities (Goulden et al., 1996; Baldocchi, 2003). The EC towers offer unprecedented opportunities to study the variability of photosynthesis on a large scale, but their footprints are still limited and they do not give the complete picture of terrestrial ecosystems.

In order to measure terrestrial photosynthesis regionally or globally and continuously, regional and global networks of micrometeorological tower sites that use eddy covariance methods to measure the exchanges of $\mathrm{CO}_{2}$ between terrestrial ecosystems and the atmosphere have been established, such as NEON (National Ecosystem Observatory Network, www.neonscience.org) in the United States, ICOS (Integrated Carbon Observatory System, www.icos- 
infrastructure.eu) in Europe and FLUXNET (http://fluxnet.ornl.gov). One of the principal objectives and challenges for the EC network community has been upscaling (Xiao et al., 2008; Jung et al., 2009; Xiao et al., 2012). These tower-based observations need to be upscaled to regions, continents, or the global scale. Although there are more than 500 tower sites around the world that are operating on a long-term basis and the number is growing, these towers are far from enough to cover the world or a continent. Besides the limited footprints (several kilometers), the towers in the networks are irregularly distributed, e.g. the towers in FLUXNET are heavily biased to regions in the mid-latitudes of the northern hemisphere (Baldocchi et al., 2001; Jung et al., 2009).

Attempts to estimate photosynthesis from remotely sensed data were made after realizing that true global measurements could only be made using satellite remote sensing. Satellite estimates of terrestrial GPP or NPP commonly rely on the strong connection between photosynthesis and solar light absorption (i.e. photosynthetically active radiation, PAR). Because remote sensing provides a measure of the fraction of absorbed PAR (fPAR), it is regarded as a promising technique for global photosynthesis monitoring. However, not all the absorbed solar light is used for photosynthesis. The conversion of absorbed PAR (APAR) to photochemistry is described by the well-known photosynthetic light use efficiency (LUE) model for GPP (Monteith, 1972). In this model GPP is expressed as a product of APAR $(=\mathrm{PAR} \times \mathrm{fPAR})$ and LUE.

$$
\mathrm{GPP}=\mathrm{PAR} \times \mathrm{fPAR} \times \mathrm{LUE}
$$

Vegetation reflectance data from remote sensing are used to estimate fPAR and thus APAR (Baret and Guyot, 1991). For example, vegetation indices (VIs) such ratio vegetation index (RVI) (Pearson and Miller, 1972) and normalized difference vegetation index (NDVI) (Rouse Jr et al., 1974) are simple and effective estimates of canopy fPAR. Global products of fPAR were generated from reflectance data of several satellites, such as MODIS (Moderate Resolution Imaging Spectroradiometer) (Myneni et al., 2002) and 
AVHRR (Advanced Very High Resolution Radiometer) (Los et al., 2000). Absorption of PAR is evidently the dominant driver and the determining factor for GPP. Provided with the remotely sensed APAR, one can have a first approximation of GPP by assuming LUE to be constant.

The conversion efficiency of APAR (LUE) is nevertheless an essential variable for GPP estimating too, because it varies widely with different vegetation types and climatic conditions (Field et al., 1995; Turner et al., 2003; Running et al., 2004). Spatial and temporal dynamics of biome APAR and LUE are key variables for understanding the relationship between climate drivers and global GPP. Simulations of global GPP were conducted in advance with ecosystem models that estimate LUE for variations in vegetation types, temperature and moisture stress (Turner et al., 2003; Running et al., 2004; Heinsch et al., 2006). The general logic is first determining the theoretical potential LUE values according to land cover and then adjusting LUE values for the climatic conditions, such as air temperature and vapor-pressure deficit (VPD). The resulting LUE values and the associated vegetation types and climatic variables are organized as a biome parameter look-up table (BPLUT) for the users (White et al., 2000; Running et al., 2000; Running and Zhao, 2015).

Reflectance signals are not directly related to LUE (Grace et al., 2007). The LUE of photosynthesis responds to ambient conditions dynamically, whereas reflectance is rather stable in a short term. Some chemical reactions in photosynthesis may induce subtle changes in reflectance, however. The photochemical reflectance index (PRI) proposed by Gamon et al. (1992) as the normalized difference of reflectance at $570 \mathrm{~nm}$ and $531 \mathrm{~nm}$ $\left(\left(R_{570}-R_{531}\right) /\left(R_{570}+R_{531}\right)\right)$ shows some correlations with LUE (Nichol et al., 2000; Barton and North, 2001; Nakaji et al., 2006). The correlations are however complex and superimposed by many other factors and no conclusive relationship has been presented yet.

Solar-induced chlorophyll fluorescence (SIF) has been considered as a measure of photosynthesis over the past decades, but the detection of SIF from satellites is of much more recent date. Guanter et al. (2014) compared 
spaceborne SIF and GPP over cropland and grassland ecosystems and found a significant correlation between them. This correlation was confirmed for different ecosystems at various temporal scales (Yang et al., 2015; Sun et al., 2017). The interest in the use of satellite data of SIF for photosynthesis estimates is the basis of this thesis.

\section{Sun-induced chlorophyll fluorescence for photosyn- thesis}

\subsection{Chlorophyll fluorescence}

Chlorophyll fluorescence (ChlF) occurs during photosynthesis. Light energy absorbed by chlorophyll molecules can undergo one of three pathways: it is used to drive photosynthesis, it is dissipated as heat or re-emitted as ChlF (Maxwell and Johnson, 2000). ChlF is therefore defined as the re-emission of radiation absorbed by chlorophyll (at a longer wavelength than the excitation wavelength). It occurs within the waveband $640-850 \mathrm{~nm}$ and has peaks at 690 and $740 \mathrm{~nm}$ (Fig. 1.1). Photosynthetically active radiation (PAR) can induce ChlF. ChlF induced by solar light is SIF (also called passive ChlF), and ChlF induced by artificial light is often termed as active ChlF.

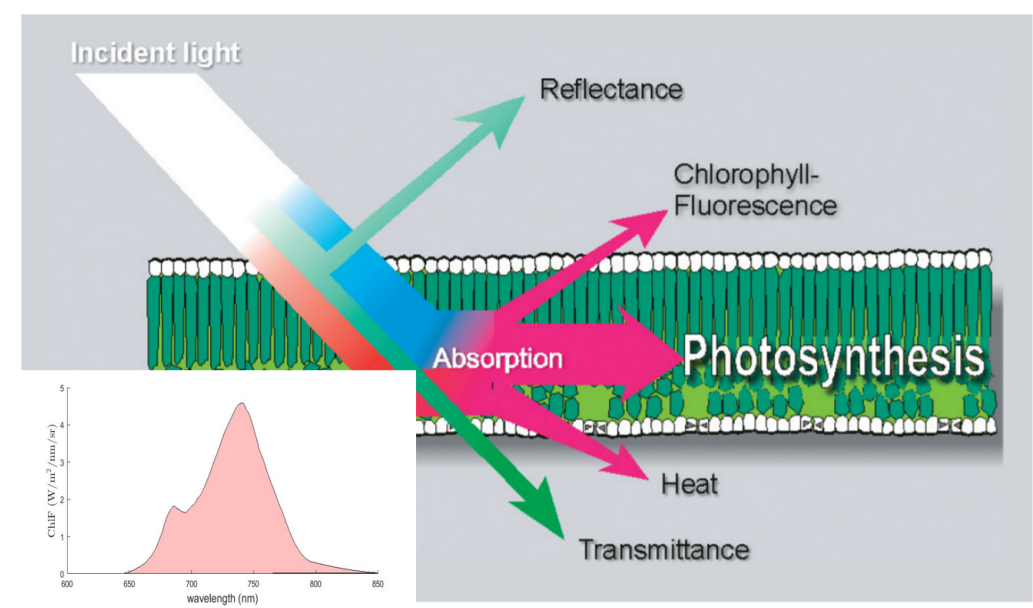

Figure 1.1: The emission of chlorophyll fluorescence of a leaf (Davidson et al., 2003) and a typical fluorescence spectrum.

ChlF is a radiative flux and it is determined by the intensity of absorbed 
energy and the conversion efficiency between absorbed radiation (i.e. APAR) and ChlF radiation (Eq. 1.3). This efficiency is called fluorescence emission efficiency $\left(\epsilon_{F}\right)$, fluorescence quantum efficiency or fluorescence quantum yield. ChlF or SIF mentioned in this thesis refer to a flux in energy units unless indicated otherwise:

$$
\mathrm{ChlF}=\mathrm{APAR} \times \epsilon_{F}
$$

\subsection{Connection between ChIF and photosynthesis}

The existence of a functional relationship between ChlF and photosynthesis has been elucidated by both laboratory and field experiments. ChlF emitted by the photosynthetic machinery can provide a direct measure of the actual functional status of vegetation (Schreiber et al., 1986; Genty et al., 1989; Baker, 2008). Temporally averaged satellite-based ChlF data appear to improve the estimation of GPP (Frankenberg et al., 2011; Guanter et al., 2014; Migliavacca et al., 2017), and provide an indication of plant stress (Ač et al., 2015; Rossini et al., 2015).

At photosystem level, the principle underlying the relationship between ChlF and photosynthesis is straightforward by looking into the quenching mechanisms of excited chlorophyll. Fluorescence reduces due to photochemical quenching (PQ) and non-photochemical quenching (NPQ). Generally, $\mathrm{ChlF}$ is inversely related to photosynthesis (PQ), except when NPQ of fluorescence (thermal dissipation) occurs (Pedrós et al., 2008). More specifically, the process of ChlF emission, together with heat dissipation, competes with photosynthesis for the same excitation energy, such that any increase in the efficiency of one will result in a decrease in the summed efficiency of the other two (Eq. 1.4). Therefore, by measuring the fluorescence emission efficiency $\left(\epsilon_{F}\right)$, information about changes in the efficiency of photochemistry and heat dissipation $\left(\epsilon_{H}\right.$ and $\left.\epsilon_{P}\right)$ can be obtained (Genty et al., 1989; Baker, 2008). It is noted that $\epsilon_{P}$ is the fraction of absorbed light for photochemistry (unitless). It can be converted to LUE with which this absorbed light is converted to 
fixed carbon (i.e. production per absorbed energy).

$$
\epsilon_{F}+\epsilon_{H}+\epsilon_{P}=1
$$

The link between ChlF and photosynthesis on the canopy, regional or ecosystem scale relies on the fact that both of ChlF and GPP are products of absorbed radiation of vegetation canopies (Eqs. 1.2 and 1.3). ChlF is determined by APAR and photosynthetic functioning and is a probe of photosynthetic functioning (LUE) and light absorption (APAR), and thus GPP.

\subsection{Measurements of ChIF}

Modern studies of ChlF started with the observation of the Kautsky effect (ChlF induction curves) in 1931 by Kautsky, although the first record of ChlF was as early as 1834 (Krause and Weis, 1991; Baker, 2008). This effect describes the phenomenon of a typical variation on ChlF of dark-adapted photosynthesizing cells that are illuminated with continuous light: ChlF first increases to a peak and then decreases to a steady state.

More advanced understanding of $\mathrm{ChlF}$ and its relationship with photosynthesis is largely derived from studies using pulse-amplitude modulated (PAM) fluorometry (Schreiber et al., 1986). In this instrument, a modulated weak light is added on top of the ambient light (which can be either natural light or artificial light), and the returning ChlF, which is also oscillating, is detected. Because the intensity of the modulated light (i.e. APAR in Eq. 1.3) is held constant, the PAM ChlF signal is proportional to fluorescence emission efficiency $\left(\epsilon_{F}\right)$.

A saturating light source is used in the PAM fluorometry apart from the modulated light. It provides a way to study the ChlF quenching. The modulated light allows measuring steady-state $\epsilon_{F}\left(F_{t}\right)$, and the saturated light allows measuring the maximal level of $\epsilon_{F}\left(F_{m}\right)$ by blocking the photochemistry pathway (note: PAM measurements are usually represented by a symbol ' $F$ '). It can be shown that the relative difference of ChlF measurements at the two states (i.e. absence or presence of saturated light) is the photosynthetic 
efficiency $\left(\epsilon_{P}\right)$. The analysis of the quenching of fluorescence leads to the famous equation linking fluorescence with steady-state photosynthesis (Genty et al., 1989).

$$
\epsilon_{P}=\left(F_{m}-F_{t}\right) / F_{m}
$$

PAM ChlF measurements are powerful tools to study photosynthetic light partitioning and plant physiology at leaf level. However, the use of artificial light (the modulated and saturating light) makes the PAM technique unsuitable for remote sensing applications.

\subsection{Remote sensing of SIF}

With the development of instruments and improvements of retrieval approaches, SIF has been measured from various remote sensing platforms ranging from tower-based (Moya et al., 2004; Guanter et al., 2013) to aircraftbased (Zarco-Tejada et al., 2009, 2012; Rascher et al., 2015) and satellite-based platforms (Joiner et al., 2011, 2013; Guanter et al., 2016). Global maps of SIF have been measured by the Greenhouse Gases Observing Satellite (GOSAT) (Frankenberg et al., 2011), the Global Ozone Monitoring Experiment-2 satellite (GOME-2) (Joiner et al., 2013) and the Orbiting Carbon Observatory satellite (OCO-2) (Frankenberg et al., 2014). Additionally, the FLuorescence EXplorer (FLEX) satellite mission has been selected as the 8th Earth Explorer mission of the European Space Agency (ESA). FLEX will be the first satellite mission dedicated to SIF observation, and will allow retrieving the full spectrum of fluorescence with high spatial resolution (Drusch et al., 2016, 2017).

The key of remote sensing SIF is to separate observed signals into a reflected component and a fluorescence component. SIF is a weak flux and is mixed with a reflected flux. It constitutes only a very small additive offset (typically $<1 \%-2 \%$ ) to the overall reflected sunlight (Meroni et al., 2009; Berry, 2012). It is challenging to differentiate SIF from reflected signals in most bands. Fortunately, in the Fraunhofer lines (i.e. absorption features of the optical spectrum of the sun) emitted fluorescence signals are enhanced 
with respect to reflected solar radiation, and thus SIF signals are relatively amplified and can be retrieved. The commonly used atmospheric Fraunhofer lines are two oxygen absorption features at $687 \mathrm{~nm}\left(\mathrm{O}_{2}-\mathrm{B}\right)$ and $760 \mathrm{~nm}$ $\left(\mathrm{O}_{2}-\mathrm{A}\right)$, representing red and far-red SIF.

Various methods have been developed to retrieve SIF from spectral measurements. Most of the approaches in the literature on SIF retrieval are based on the simplistic Fraunhofer Line Depth (FLD) principle (Plascyk, 1975), the modified FLD (3FLD) (Maier et al., 2003), the improved FLD (iFLD) (Alonso et al., 2008). More advanced SIF retrieval methods such as spectral fitting methods (SFM) have been proposed for a more robust and accurate retrieval (Meroni et al., 2010; Guanter et al., 2010; Damm et al., 2014)

SIF has been a widespread and exciting signal for monitoring vegetation. The availability, quality, and spatiotemporal coverage of SIF data are expected to increase drastically over the next few years (Porcar Castell et al., 2014). More SIF data from various platforms are available and need to be explored. Models are needed for this purpose.

\subsection{Modeling of SIF}

Efforts on modeling SIF from photosynthetic level to leaf and canopy level have been made along with observations. At photosynthetic level, the efficiencies of the three pathways of absorbed energy are regulated by ambient conditions and vary with plant functioning types. The photochemistry pathway is well-studied earlier than ChlF by using the gas exchange techniques. Nearly four decades ago Farquhar, von Caemmerer and Berry developed a quantitative photosynthesis model for photosynthetic rates (the FvCB model) (Farquhar et al., 1980). Thanks to PAM techniques, ChlF partitioning and photosynthetic efficiency was later linked (Schreiber et al., 1986; Genty et al., 1989). A semi-empirical photosystem based energy partitioning model was later developed by (Rosema et al., 1998), in which fluorescence emission efficiency and photosynthetic efficiency are functions of PAR. Van der Tol et al. (2014) further explored the empirical relationship between fluorescence 
emission efficiency and photosynthetic efficiency and developed a more advanced photosystem energy partitioning model. This model simulates the response of energy partitioning to various factors, including leaf temperature, $\mathrm{CO}_{2}$ concentration, PAR and stomatal conductance.

Remote sensing only measures a portion of the total emitted SIF by photosystems due to re-absorption and scattering effects. Considering these effects, SIF observed from remote sensing can be expressed as

$$
\mathrm{SIF}=\mathrm{APAR} \cdot \epsilon_{F} \cdot \sigma_{F}
$$

where SIF refers to remotely sensed signals and $\sigma_{F}$ is scattering coefficient of the emitted SIF and is the ratio between observed and emitted fluorescence radiation. The efficiency can be predicted with the energy partitioning model in photosystems, while the absorption of PAR and scattering of emitted SIF are determined by the radiative transfer of incident light and emitted SIF, respectively.

Radiative transfer models (RTMs) of SIF have been developed for upscaling and downscaling fluorescence signals among photosynthetic level, leaf, and canopy level. Most of these models are adaptations or extensions of the existing RTMs for simulating reflectance and transmittance. Pedrós et al. (2010) published FluorMODleaf simulating ChlF emission by plant leaves and further improvements led to the Fluspect model (Vilfan et al., 2016). The models are an extension of PROSPECT (Jacquemoud and Baret, 1990), a widely used leaf optical properties model that simulates leaf reflectance and transmittance. Meanwhile, Miller et al. (2005) developed FluorSAIL that includes fluorescence radiative transfer in the classic canopy reflectance model SAIL (Verhoef, 1984, 1985).

SCOPE (Soil-Canopy-Observation of Photosynthesis and Energy fluxes) combines RTMs and biochemical models (Van der Tol et al., 2009). The basis of SCOPE is the classic SAIL radiative transfer models originally published by Verhoef (1984). The combination of the canopy SAIL model and the leaf PROSPECT model yielded the well-known PROSAIL (Jacquemoud, 1993; Jacquemoud et al., 1995; Jacquemoud et al., 2009). Further inclusion of 
fluorescence radiative transfer led to FluorMODleaf and FluorSAIL. The modelling of thermal radiative transfer (Verhoef et al., 2007) allows implementing an energy balance module in SCOPE that simulates the necessary micrometeorological variables for driving the biochemical model of Van der Tol et al. (2014). The partitioning of absorbed radiation of each individual leaf can be computed and leaf fluorescence emission and photosynthesis can be modeled. Aggregation of leaves' photosynthesis yields canopy photosynthesis, and top-of-canopy (TOC) SIF is predicted with the radiative transfer of emitted fluorescence.

SCOPE is a 1D model that considers leaves in a canopy which have identical optical properties. Recently, 3D models have also been developed but are mere RTMs, e.g. the Discrete Anisotropic Radiative Transfer (DART) (Gastellu-Etchegorry et al., 2017) and FluorFLIGHT (Hernández-Clemente et al., 2017).

\section{Challenges in photosynthesis monitoring from SIF}

The existence of a functional relationship between SIF and photosynthesis is definite. We, however, have to be aware that the SIF-photosynthesis relationship is generally complex. The relationship between SIF from remote sensing and GPP is merely empirical and the exact relationship remains unclear. We are in a position to move beyond the mere empirical observation of SIF-photosynthesis relationship and more work needs to be done to unravel the full potential of SIF measurements.

The most obvious challenge is at mechanistic level: how $\epsilon_{F}$ is exactly linked with $\epsilon_{P}$. Their relationship is clear only if the third pathway (heat dissipation) is known. In most studies on correlating SIF with GPP, $\epsilon_{P}$ is assumed to be linear with $\epsilon_{F}$ (Guanter et al., 2014; Guan et al., 2016; Migliavacca et al., 2017) due to lack of a mechanistic link between steady-state $\epsilon_{F}$ and $\epsilon_{P}$

SIF is ought to be better interpreted before linked with photosynthesis. SIF from remote sensing is the product of three processes: (1) the absorption of PAR by chlorophyll (fPAR), (2) the re-emission of part of this absorbed 
radiation as fluorescence $\left(\epsilon_{F}\right)$, and (3) the scattering and re-absorption of fluorescence in the canopy $\left(\sigma_{F}\right)$. Of these three factors, (1) has a direct relationship with GPP and indirectly regulates photosynthetic efficiency $\epsilon_{P}$, (2) has a direct relationship with $\epsilon_{P}$ and indirectly regulates GPP, while (3) is unrelated to either.

The key problem is the scattering of SIF in the canopy. The scattering of SIF is an interference in the SIF-GPP relationship. GPP is functionally related to SIF production of the whole canopy rather than TOC SIF, which is only a portion of total SIF production. The use of SIF for photosynthetic production (GPP) requires downscaling from remotely sensed SIF to canopy SIF production. The main problem in this downscaling is how to quantify the scattering of SIF from remote sensing measurements. Scattering of SIF is determined by leaf optical properties and canopy structure, and sensitive to sun-observer geometry (Porcar Castell et al., 2014). RTMs provide a way to predict scattering, but only if the canopy and observational conditions are pre-defined. Biophysical and biochemical variables of canopy usually are desired parameters of remote sensing rather than input parameters.

The light absorption is another problem. Retrievals of physiological functioning traits from SIF require removing the non-physiological regulation from TOC SIF. Studies show that a substantial variability of SIF is due to canopy structure and sun-observer geometry rather than physiological variation (Porcar Castell et al., 2014; Van der Tol et al., 2016; Verrelst et al., 2016; Liu et al., 2016). Fluorescence emission efficiency is a 'pure' quantity for photosynthetic functioning. The retrieval of this efficiency requires the correction of the light absorption (process (1)) and scattering of emitted SIF (process (3)). They are affected by canopy structure and sun-observer geometry. Again, RTMs are useful to predict them, but the same problems as discussed above exist. The difficult task is effectively analyzing the three processes with remote sensing measurements (e.g. TOC reflectance and SIF).

In summary, the main challenges of using SIF for photosynthesis monitoring are summarized as follows.

1. Quantification of the scattering of emitted SIF, 
2. Separation of non-physiological and physiological regulations on SIF,

3. Mechanistic link between fluorescence emission efficiency and photosynthetic efficiency.

\section{Objectives and organization of the thesis}

The main objective of this thesis is to quantitatively analyze the three processes (i.e. light absorption, fluorescence emission and re-absorption of fluorescence) that affect TOC SIF observations. Efforts on separation of plant physiological and non-physiological regulation of SIF, and on radiative transfer modeling are made to consolidate the interpretation of SIF. This thesis aims to do this via the following steps:

1. Simulating the effects of light absorption and re-absorption of fluorescence by using the SCOPE model and reflectance data (Chapter 2),

2. Quantification of re-absorption and scattering of emitted SIF by using reflectance (Chapter 3),

3. Separation of non-physiological and physiological regulations on SIF by using a reflectance index (Chapter 4),

4. Improvement of SCOPE to better interpret SIF signals (Chapter 5). 



\title{
Interpreting SIF measurements by using radiative transfer models*
}

\author{
-A case of heat wave study
}

${ }^{*}$ This chapter is based on: Yang, P., van der Tol, C., Verhoef, W., Damm, A., Schickling, A.,Kraska, T.,Muller, O.,Rascher, U., 2018. Response of Crops to a Heat Wave: Insights from Airborne based Reflectance and Chlorophyll Fluorescence Measurements. Remote Sensing of Environment, under review. 


\section{Abstract}

Weather extremes affect crop production and pose a threat to food security. Crop monitoring and early plant stress detection can facilitate an improved crop management, thus alleviating this threat. The growing availability of global measurements of sun-induced chlorophyll fluorescence (SIF) can help improving crop monitoring in the near future, especially the monitoring of photosynthetic activity. In this study, we quantitatively analyzed airborne (HyPlant) reflectance and SIF data acquired over an agricultural farm in Germany on two days, before and during a heat wave in summer 2015 with maximum temperatures of $26^{\circ} \mathrm{C}$ and $34^{\circ} \mathrm{C}$, respectively. Reflectance spectra and SIF responded to the high temperature differently across investigated crops. Inversions with the combined photosynthesis and energy balance model SCOPE showed that these responses were due to changes in canopy structure, leaf water content and photosynthetic functioning. We demonstrate that the use of reflectance data and radiative transfer models provides a way to disentangle structural and physiological responses of vegetation. This opens new pathways to compensate for vegetation structural effects on TOC SIF, and thus track photosynthesis functioning status in response to heat stress. The combination of reflectance and SIF enables the early detection of canopy structural and plant physiological response to environmental conditions. This provides valuable information to advance analysis of environmental stress response of vegetated ecosystems, in particular, their response to rising temperature. 


\section{Introduction}

Early detection of high temperature stress of vegetation is crucial for precision agriculture and global food security. High temperatures and associated effects such as water deficit and excessive radiation levels may affect plant development (Levitt et al., 1980; Chaves et al., 2002; McDonald and Paulsen, 1997). A study by Reyer et al. (2013) found a substantial impact of extreme temperature events on plant phenology and an increased vulnerability of plant-water relations. Reduced ecosystem productivity has been reported during the heat wave in Europe in 2003 (Ciais et al., 2005; Reichstein et al., 2007). Time series analysis (Rahmstorf and Coumou, 2011) and climate modelling (Vasseur et al., 2014) show that an increase in the frequency and severity of heat waves across the globe is a component of present climate change.

Remote sensing provides the technology to identify stress at large scales before the weather extremes cause irreversible damages to crops (Carter and Miller, 1994; Dobrowski et al., 2005; Zarco-Tejada et al., 2009). Information can be retrieved from reflected solar and emitted radiation (sun-induced chlorophyll fluorescence (SIF) or thermal (TIR)). These signals are determined by the biochemical, structural and functional properties of the plants (Grace et al., 2007; Zarco-Tejada et al., 2009). It is well known that the reflectance of stressed plants is qualitatively and quantitatively different from that of healthy plants (Carter and Miller, 1994; Dobrowski et al., 2005). Further, it has been found that SIF signals emitted at both $687 \mathrm{~nm}$ and $760 \mathrm{~nm}$, known as red and far red SIF, and observed with remote sensing techniques vary in response to crop stress (Ač et al., 2015; Rossini et al., 2015).

The key to linking SIF and reflectance to photosynthesis and stress is to separate the effects of canopy structure (the spatial organization of leaves, i.e. the 'architecture' of the plants) from those of leaf physiology. SIF depends on a number of factors: photochemistry in the leaf, canopy structure, the sun-observer geometry and incident light intensity (Porcar Castell et al., 2014; Rascher et al., 2015). The challenge is to identify which parameter or 
process is responsible for an observed change in SIF.

The different factors that determine SIF may be quantified with radiative transfer models (RTMs) for vegetation. These models offer an explicit connection between top-of-canopy (TOC) reflectance and SIF observations and vegetation variables (Houborg et al., 2007). A number of RTMs are capable of simulating the interaction of incident and fluorescence radiance with the leaf (Pedrós et al., 2010; Vilfan et al., 2016) and canopy (Zarco-Tejada et al., 2006; Van der Tol et al., 2009; Gastellu-Etchegorry et al., 2017; Yang et al., 2017; Hernández-Clemente et al., 2017).

Sensitivity analyses of RTMs show that the effect of canopy structural parameters on SIF is substantial (Koffi et al., 2015; Verrelst et al., 2016), and combined field measurements and modelling confirm that seasonal variations in SIF are largely driven by canopy structure (Van der Tol et al., 2016; Migliavacca et al., 2017). While simply normalizing SIF by PAR removes the effects of variation in incident light (Daumard et al., 2012), it is insufficient to separate canopy structure from leaf physiological effects on SIF.

RTMs require canopy structure and leaf properties as input, which are generally not known a priori. Therefore, model inversion (or retrieval) is needed to obtain required canopy structure and leaf properties needed for the simulation of SIF (Jacquemoud, 1993; Darvishzadeh et al., 2008). For example, Verhoef et al. (2018) retrieved canopy biophysical parameters from synthetic (i.e. simulated) top-of-atmosphere (TOA) radiances by inverting the SCOPE model ('Soil-Canopy Observation of Photosynthesis and Energy fluxes') of Van der Tol et al. (2009). With a similar approach, Van der Tol et al. (2016) retrieved key biophysical and biochemical parameters from the visible and near-infrared (i.e. 400 - $900 \mathrm{~nm}$ ) reflectance data of rice and alfalfa canopies. Both studies show that the use of the reflectance spectrum to parameterize a radiative transfer model for fluorescence, greatly improves the interpretation of SIF.

Once the canopy structure effects of (re-)absorption of SIF are 'removed' (corrected for) by means of model simulation, the SIF signal is scaled to the level of a photosystem (Grace et al., 2007; Baker, 2008; Meroni et al., 
2009), and the efficiency of the emission of fluorescence by chlorophyll in photosystems can be estimated. This efficiency scales inversely with the efficiency of energy dissipation by photochemistry $\left(\epsilon_{P}\right)$ and heat dissipation $\left(\epsilon_{H}\right)$. Hence, by measuring the fluorescence emission efficiency $\left(\epsilon_{F}\right)$, information about the efficiencies $\epsilon_{P}$ and $\epsilon_{H}$ can be obtained (Baker, 2008; Van der Tol et al., 2016).

This study aims to assess and demonstrate the sensitivity of remote sensing approaches to track canopy structural and leaf photochemical responses of crops to heat stress. We use a unique airborne dataset comprising observations of canopy reflectance and SIF took before (June 30th) and during (July 2nd) a heat wave in 2015 with the HyPlant system. HyPlant (Rascher et al., 2015) is a novel airborne spectrometer system dedicated to vegetation functional monitoring. Two spectrometers allow the estimation of red SIF at $687 \mathrm{~nm}$ and far red SIF at $760 \mathrm{~nm}$, and reflectance from $400 \mathrm{~nm}$ to $2500 \mathrm{~nm}$. The measurements of reflectance provide the opportunity for mapping canopy structure and leaf properties. The canopy structure and leaf properties are further used to compensate canopy structural effects on SIF by using SCOPE, and thus physiology parameters of vegetation are retrieved.

\section{Materials and methods}

\subsection{Overview and workflow}

Our approach is to use reflectance to quantify photosynthetic light absorption and the scattering and re-absorption of SIF, whereafter the measured SIF can be used to estimate the emission efficiency $\epsilon_{F}$, defined as the fraction of absorbed radiation by chlorophyll that is emitted as fluorescence by both photosystems.

For this purpose we express TOC SIF radiance $\left(\mathrm{Wm}^{-2} \mu \mathrm{m}^{-1} \mathrm{sr}^{-1}\right)$ as:

$$
L_{F}=\frac{1}{\pi} \mathrm{PAR} \times \Gamma_{r t} \times \epsilon_{F}
$$

where $\Gamma_{r t}\left(\mathrm{sr}^{-1}\right)$ quantifies the canopy structural contribution to SIF. $\Gamma_{r t}$ is the product of the fraction of absorbed PAR (fPAR), and scattering of 
SIF $\left(\sigma_{F}\right)$ (i.e. $\left.\Gamma_{r t}=\mathrm{fPAR} \sigma_{F}\right)$. Following Eq. 2.1, the scattering of SIF $\sigma_{F}$ is by definition the ratio of observed directional radiation $\left(\pi L_{F}\right)$ over the total emitted fluorescence $\left(\mathrm{fPAR} \times \mathrm{PAR} \times \epsilon_{F}\right)$.

Because $\Gamma_{r t}$ accounts for the radiative transfer of the incident light (i.e. PAR absorption) and emitted SIF, it is called the 'radiative transfer factor' in this study. The efficiency $\epsilon_{F}$ is referred to as the 'physiological factor', because it represents the efficiency of dissipation pathways in both photosystems.

The approach to estimate $\Gamma_{r t}$ and $\epsilon_{F}$ is illustrated in Fig. 2.1. We first retrieve vegetation parameters from TOC reflectance by inverting the combined RTM and energy balance model SCOPE. Next, we use the model and retrieved parameters to simulate the canopy structural contribution $\left(\Gamma_{r t}\right)$ to the SIF measurements. This finally enables us to solve $\epsilon_{F}$ from measured SIF $\left(L_{F}\right)$, measured PAR, and Eq 2.1.

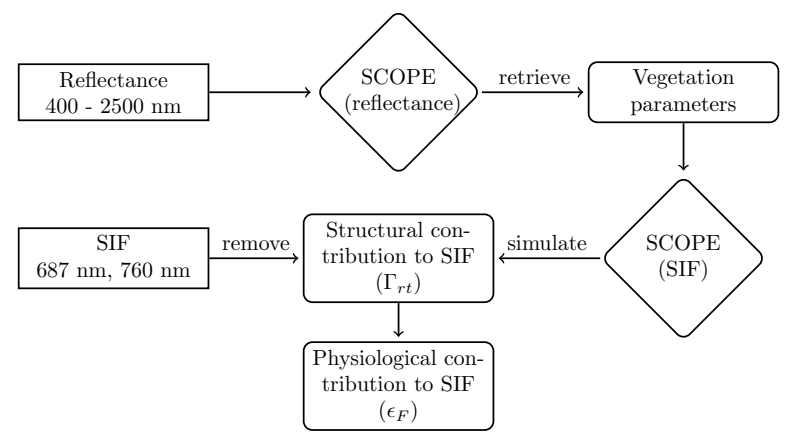

Figure 2.1: Workflow of interpretation of HyPlant reflectance and SIF data by using SCOPE.

\subsection{Study area}

The study area is located in the agricultural experimental research station Campus Klein Altendorf of the University of Bonn, Germany (50³7' $\left.\mathrm{N}, 6^{\circ} 59^{\prime} \mathrm{E}\right)$. The average altitude of the field is $65 \mathrm{~m}$ above mean sea level. The mean annual precipitation is $603 \mathrm{~mm}$ and the mean annual temperature is $9.4^{\circ} \mathrm{C}$. All analyses were performed in a 3 ha $(100 \mathrm{~m} \times 300 \mathrm{~m})$ experimental 
field (Fig. 2.2). The study area faced a heat wave during July in 2015 (Dong et al., 2016) with severity comparable to the summer Europe heat waves in 2003 and 2010 (Beniston, 2004; Barriopedro et al., 2011; Christidis et al., 2015). The heat wave started on July 1st and lasted to July 5th in 2015 with maximum temperatures exceeding $30^{\circ} \mathrm{C}$ on each of these days.

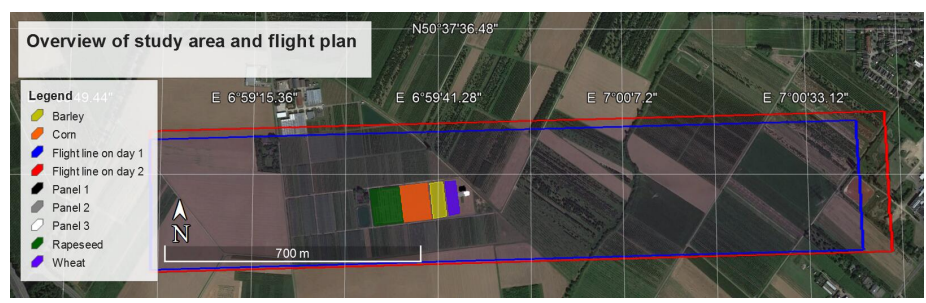

Figure 2.2: Overview of the study area and the flight plan before (June 30th, day 1) and during (July 2nd, day 2) the heat wave. The crops investigated in the study and three reference panels are marked with polygons. The background image was acquired on 24th August 2016 (from Google Earth).

At the study site, four crop types, notably corn (Zea mays L.), winter wheat (Triticum aestivum L.), winter barley (Hordeum vulgare L.) and rapeseed (Brassica napus L.), were grown using common field rotation practices. These four crops are the main crop types across western Germany. The soil and crops were treated according to the agricultural practices of the region with the aim to provide a spatially homogeneous plot design. Three (black, grey and white) reference panels were placed next to the experimental field.

\subsection{Airborne experiment}

The airborne campaigns were conducted on June 30th (day 1, one day before the heat wave) and July 2nd (day 2, during the heat wave). The air temperature was $26.6^{\circ} \mathrm{C}$ on day 1 and $33.7^{\circ} \mathrm{C}$ on day 2 during data acquisition. Both flights were carried out between 15:00 and 16:00 (local time, UTC+2) under perfectly clear sky conditions, at an altitude of $512 \mathrm{~m}$ above sea level. Flights were carried out at similar solar zenith angles (i.e. $38^{\circ}$ and $34^{\circ}$ ). Incident photosynthetically active radiation (PAR) was similar as well (i.e. 278 and $297 \mathrm{~W} \mathrm{~m}^{-2}$ ). 
The used HyPlant sensor was designed for vegetation monitoring (Rascher et al., 2015). It consists of the dual-channel (DUAL) module and the fluorescence (FLUO) module. The DUAL module measures contiguous spectra from 380 to $2500 \mathrm{~nm}$ with a spectral resolution of $3 \mathrm{~nm}$ in visible and near infrared region, and $10 \mathrm{~nm}$ in short-wave infrared region. The FLUO module measures in 1024 contiguous spectral bands from 670 to $780 \mathrm{~nm}$, with a spectral resolution of $0.25 \mathrm{~nm}$. A set of representative measurements from the two modules is shown in Fig. 2.3. Both DUAL and FLUO module (i.e. two imagers), together with the thermal camera Variocam (InfraTec, Germany), were mounted on a single platform with the mechanical capability to align the field of view (FOV). The thermal camera was connected to a laptop via GigaEthernet and approached with the IRBIS@3 software (Infratec, Germany), which allows real-time tracking of the measurements and correction of the absolute temperature by setting of emissivity, background temperature, ambient air temperature, air humidity and objects distance.

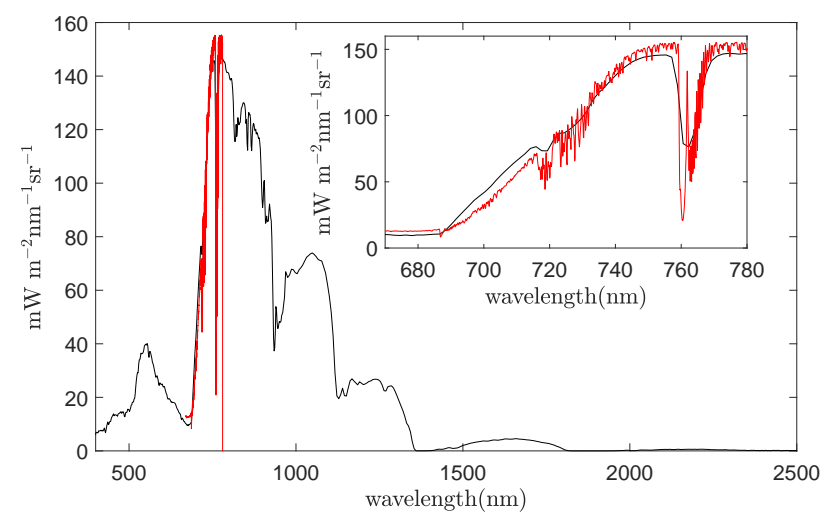

Figure 2.3: Representative radiance measurements of vegetation from the DUAL (black) and the FLUO (red) module, respectively.

Supporting atmospherical parameters were acquired with the sun photometer MICROTOPS II (Solar Light, the USA) every 5 minutes during the time of overflights and were later used for atmospheric correction. Additional recorded meteorological parameters are listed in Table 2.1. 
Table 2.1: The meteorological conditions during the airborne campaigns before and during the heat wave.

\begin{tabular}{lcc}
\hline Parameters & 30th June (Day 1) & 2nd July (Day 2) \\
\hline DOY (day of year) & 182 & 184 \\
Acquisition time $($ local $)$ & $15: 51$ & $15: 16$ \\
Solar zenith $\left(\theta_{s}\right.$, degree) & 38 & 34 \\
Solar azimuth $\left(\psi_{s}\right.$, degree) & 237 & 224 \\
Air temperature $\left(T_{a},{ }^{\circ} \mathrm{C}\right)$ & 26.6 & 33.7 \\
Shortwave radiation $\left(\mathrm{W} \mathrm{m}{ }^{-2}\right)$ & 756 & 808 \\
Wind speed $\left(\mathrm{m} \mathrm{s}^{-1}\right)$ & 3 & 2 \\
Air pressure $(\mathrm{hPa})$ & 997 & 996 \\
\hline
\end{tabular}

\subsection{Calculation of reflectance and SIF}

Reflectance was calculated from data of the DUAL module, and SIF from data of the FLUO module. The detailed processing has been described in Rascher et al. (2015), details of the fluorescence retrieval can be found in Damm et al. (2014) and Wieneke et al. (2016). In the following, we outline the main procedure.

Data preprocessing included several steps: Measured raw data (digital numbers) of both modules were converted to calibrated at-sensor radiance data using the radiometric calibration coefficients provided by the manufacturer. Resulting at-sensor radiance images were then geometrically rectified using navigation data recorded by the GPS/IMU unit and resized to a spatial grid of $0.5 \mathrm{~m} \times 1 \mathrm{~m}$. DUAL data were atmospherically corrected using an atmospheric and topographic correction approach for flat terrain (ATCOR-4) (Richter and Schlapfer, 2012). ATCOR-4 is based upon the atmospheric radiative transfer code MODATRAN-5 (Berk et al., 2005) to pre-calculate look-up tables (LUT) of atmospheric functions such as transmission, spherical albedo, path scattered radiance. The atmosphere type and aerosol model were set to mid-latitude summer and a rural aerosol model. Solar position, ground elevation, and sensor elevation were parameterized to the actual measurements during data acquisition. This parameterization was combined with estimates of atmospheric water vapor and aerosol optical thickness from MICROTOPS II to account for atmospheric absorption and scattering effects 
and eventually retrieve TOC radiance and TOC reflectance.

TOC fluorescence at $687 \mathrm{~nm}\left(F_{687}\right)$ and at $760 \mathrm{~nm}\left(F_{760}\right)$ were retrieved from at-sensor radiance measured by the FLUO module. The fluorescence retrieval was based on the iFLD method introduced by Alonso et al. (2008) as a modification of the original FLD method (Plascyk, 1975). For this study, we exploited the two oxygen absorption lines (i.e. $\mathrm{O}_{2}$-A and $\mathrm{O}_{2}$-B) located at $687 \mathrm{~nm}$ and $760 \mathrm{~nm}$ and further updated the method to make it applicable for airborne use. The main update comprises of the use of non-fluorescent reference surfaces to correct potential inaccuracies in estimated atmospheric functions. Such errors can occur if atmospheric parameters are not exactly known or slight sensor artifacts remain (cf. Damm et al. (2014) and Wieneke et al. (2016) for details). Accurate fluorescence retrievals from airborne remote sensing measurements are challenging but the reliability of obtained SIF using this method was confirmed by validation activities considering ground fluorescence measurements (Rossini et al., 2015; Rascher et al., 2015)

Normalized difference vegetation index (NDVI) (Rouse Jr et al., 1974) and photochemical reflectance index (PRI) (Gamon et al., 1992), as proven non-invasive early indication of plant stress, were computed from the reflectance data as follows:

$$
\begin{aligned}
& \mathrm{NDVI}=\frac{R_{780-785}-R_{680-685}}{R_{780-785}+R_{680-685}} \\
& \mathrm{PRI}=\frac{R_{531}-R_{570}}{R_{531}+R_{570}} .
\end{aligned}
$$

where $R$ refers to reflectance and numbers indicate wavelength in nanometres.

\subsection{Models}

SCOPE consists of one leaf RTM, three canopy RTMs, a biochemical model, a soil reflectance model and an aerodynamic model. These models are internally connected. We briefly introduce the models (combinedly) used in this study. The details of soil reflectance model have been described in Verhoef et al. (2018), details of the biochemical model can be found in 
Table 2.2: The ranges and initial values of the key parameters used in SCOPE

\begin{tabular}{|c|c|c|c|c|}
\hline Parameter & Interpretation & Unit & Range & Initial value \\
\hline & \multicolumn{4}{|l|}{ Soil: BSM (Verhoef et al., 2018) } \\
\hline$B$ & Soil brightness & - & $0-0.9$ & 0.5 \\
\hline latitude $(\varphi)$ & Soil spectral latitude & - & $10-60$ & 45 \\
\hline longitude $(\lambda)$ & Soil spectral longitude & - & $10-50$ & 40 \\
\hline$S M_{p}$ & $\begin{array}{l}\text { Soil moisture volume percentage } \\
\text { Leaf model: Fluspect (Vilfan et al }\end{array}$ & $\begin{array}{l}- \\
., 2016)\end{array}$ & $5-55$ & 20 \\
\hline$C_{a b}$ & Chlorophyll $a+b$ content & $\mu \mathrm{g} \mathrm{cm}^{-2}$ & $0-80$ & 40 \\
\hline$C_{d m}$ & Leaf mass per unit area & $\mathrm{g} \mathrm{cm}^{-2}$ & $0-0.02$ & 0.01 \\
\hline$C_{w}$ & Equivalent water thickness & $\mathrm{cm}$ & $0-0.1$ & 0.02 \\
\hline$C_{s}$ & Brown pigments & a.u. & $0-1$ & 0.5 \\
\hline$C_{c a}$ & Carotenoid content & $\mu \mathrm{g} \mathrm{cm}^{-2}$ & $0-30$ & 10 \\
\hline$N$ & Leaf structure parameter & - & $0-3$ & 1.5 \\
\hline$\epsilon_{F}$ & Fluorescence emission efficiency & - & $0-0.1$ & 0.01 \\
\hline & \multicolumn{4}{|c|}{ Canopy models: RTMo and RTMf (Van der Tol et al., 2009) } \\
\hline LAI & Leaf area index & $\mathrm{m}^{2} \mathrm{~m}^{-2}$ & $0-7$ & 3 \\
\hline LIDFa & Leaf inclination determination a & - & $-1-1$ & -0.35 \\
\hline LIDFb & Leaf inclination determination $b$ & - & $-1-1$ & -0.15 \\
\hline
\end{tabular}

Van der Tol et al. (2014), the leaf RTM can be found in Vilfan et al. (2016) and the canopy RTMs can be found in Van der Tol et al. (2009).

\subsubsection{BSM soil reflectance model}

Brightness-Shape-Moisture (BSM) model simulates soil reflectance. It is an adaptation and extension of the 'Global Soil Vector' (GSV) model (Chongya Jiang, 2012), which fits any given dry soil reflectance spectrum by using several "basis spectra". BSM separates soil brightness effects, soil moisture effects and spectral shape effects on soil reflectance (Verhoef et al., 2018). The model requires soil brightness $(B)$, soil moisture $\left(S M_{p}\right)$, and two spectral-shape related parameters $(\varphi$ and $\lambda$ ) (Table 2.2).

\subsubsection{The biochemical model}

The biochemical model developed by Van der Tol et al. (2014) model is a photosynthetic energy distribution model, and is based on the conventional Farquhar et al. (1980) and Collatz et al. (1992) photosynthesis model. It simulates the efficiency $(\epsilon)$ of fluorescence emission $(\mathrm{F})$, photochemistry $(\mathrm{P})$ and heat dissipation $(\mathrm{H})$. The efficiencies are determined by (1) absorbed PAR, (2) leaf temperature, (3) the maximum rate of carboxylation $\left(V_{c m a x}\right)$, 
and (4) other ambient environmental conditions, such as air pressure and concentration of $\mathrm{O}_{2}$ and $\mathrm{CO}_{2}$ in the leaf boundary layer.

\subsubsection{Fluspect model}

Fluspect (Vilfan et al., 2016), which is based on PROSPECT (Jacquemoud and Baret, 1990), simulates leaf reflectance, transmittance, and fluorescence emission of both forward (shaded) and backward (illuminated) side.

In order to simulate leaf reflectance and transmittance, one needs to provide chlorophyll content $\left(C_{a b}\right)$, carotenoid content $\left(C_{c a}\right)$, leaf water content $\left(C_{w}\right)$, dry matter content $\left(C_{d m}\right)$, brown pigment content $\left(C_{s}\right)$, and leaf structure parameter $(N)$ as input. These inputs are identical to those in PROSPECT. Simulation of SIF emission of a leaf requires the input of incident radiation to the leaf and fluorescence emission efficiency $\left(\epsilon_{F}\right)$. The efficiency is spectral dependent ranging from $640 \mathrm{~nm}$ to $850 \mathrm{~nm}$ and its distribution in wavelength (i.e., its integration over wavelengths is unity) is calibrated to leaf-level measurements (Vilfan et al., 2016) (see Fig. S1 in the supplementary materials). The overall efficiency is estimated with the semi-empirical model of Van der Tol et al. (2014) and distributed over different wavelengths following Vilfan et al. (2016).

\subsubsection{Canopy radiative transfer models}

At the canopy level, RTMo, RTMf and RTMt, which are three SAIL (Verhoef, 1984) based models, compute the radiative transfer of incident radiation, emitted fluorescence and thermal radiation, respectively. RTMo and RTMf will be introduced since they are sufficient to interpret TOC reflectance from $400 \mathrm{~nm}-2500 \mathrm{~nm}$ and SIF measured by HyPlant.

RTMo calculates the fate of incident radiation and absorbed radiation of each leaf within a canopy. It provides TOC reflectance simulations, and absorbed PAR of each leaf orientation class in different position in the canopy. The number of leaf orientation classes considered is 468 , comprising the combinations of 13 leaf's normal zenith angles, 36 leaf's azimuths with 
respect to the sun. The leaf inclination distribution is parametrized with two (a, b) parameters, which control the mean leaf zenith angle and the bimodality of the distribution (Verhoef, 1984). Average leaf angle (ALA) can be calcuated from leaf inclination distribution function parameter a (LIDFa) (Verhoef, 1998).

$$
\mathrm{ALA}^{\circ}=45-360 \times \operatorname{LIDFa} \frac{1}{\pi^{2}}
$$

SIF emission from each individual leaf can be simulated by Fluspect, using leaf APAR from RTMo and fluorescence emission efficiency from the biochemical model. Note that simulation of the efficiency requires leaf temperature as input which is predicted by an energy balance routine that includes RTMt and the aerodynamic model. RTMf simulates radiative transfer of emitted SIF by leaves in a canopy and TOC SIF.

\subsection{Approach of retrieving vegetation parameters from TOC reflectance}

\subsubsection{Retrieval of base soil properties}

We retrieved soil properties of the study area from reflectance spectra of bare soil. Some of the retrieved parameters were used as prior information in the retrieval of vegetation parameters from TOC reflectance to reduce the ill-posedness of the retrieval problem. In principle, it is possible to retrieve leaf and canopy parameters, and soil parameters together from TOC reflectance of vegetation (Verhoef et al., 2018), but due to the large number of free parameters, the model inversion may not effectively separate the contribution from soil and from vegetation to TOC reflectance. We assumed reflectance of soil beneath the canopies differentiate from each other by soil moisture $\left(S M_{p}\right)$. The soil parameters $B, \varphi$ and $\lambda$ were kept to the values retrieved from the reflectance of bare soil. In this way, we reduced the uncertainty in retrieving vegetation parameters.

Twenty soil spectra were taken from the images on each day as shown in Fig. 2.4. For the retrieval of soil parameters from each of these reflectance spectra, we used the Numerical Optimization (NO) method. The NO method 
aims at minimizing a cost function, which quantifies the difference between measured and simulated signals by successive changing the values of the input parameters. For the retrieval from soil reflectance, the BSM model was iteratively executed, each time with different parameter values and stopped until obtaining a satisfying fitting to the measured soil spectra. The cost function is

$$
f_{\text {soil }}=\left(R_{s}-R_{m}\right)^{T}\left(R_{s}-R_{m}\right)
$$

where $R_{s}$ is simulated (soil) reflectance, and $R_{m}$ is the measured (soil) reflectance in all bands of the spectra between 400 and $2500 \mathrm{~nm}$ (i.e. 623 bands). We used the function 'Isqnonlin' of the optimization toolbox of Matlab R2017a, selecting a Trust Region algorithm for updating parameter values after each iteration step, and iteration stopped when the improvement of the cost function $\left(f_{\text {soil }}\right)$ was less than $10^{-3}$. The ranges and initial values used are shown in Table 2.2 .

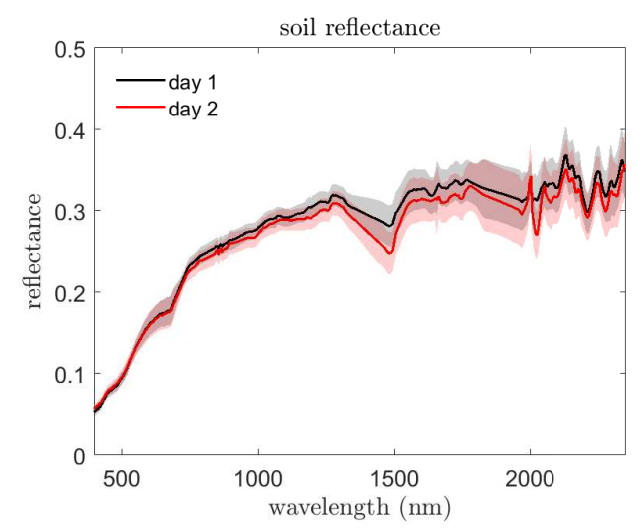

Figure 2.4: Bare soil reflectance on day 1 and on day 2. The buffers represent variation (i.e. standard deviation) in the selected pixels.

\subsubsection{Retrieval of vegetation parameters}

We retrieved leaf biophysical parameters and canopy structure parameters from TOC reflectance spectra of vegetation canopies on both days. Retrievals from reflectance before and during the heat event were conducted independently. For inversion, we again used the NO method and minimized 
the cost function (Eq. 2.5), but now using the reflectance of vegetation canopies and the canopy reflectance model (RTMo).

The reflectance simulation requires the parameters listed in Table 2.2 except for fluorescence emission efficiency. Besides these parameters, TOC reflectance is also affected by the sun-observer geometry and the fraction of direct solar light of the total incident radiation. The solar zenith angles were calculated according to the acquisition time and location, which was $38^{\circ}$ for day 1 and was $34^{\circ}$ for day 2 . We assumed the sensor view angle as spatially constant on the image and in nadir direction although the HyPlant field of view ranges from $+/-14^{\circ}$. For a nadir view, the azimuth angle is arbitrary. The spectra of incoming direct solar light and diffuse light were obtained from MODTRAN 5. The freedom of the optimization was further reduced by using the retrieved soil properties $(B, \varphi$ and $\lambda)$. Therefore, fitting one reflectance spectrum requires tuning 10 parameters $\left(S M_{p}, C_{a b}, C_{d m}, C_{w}\right.$, $C_{c a}, N$, LAI, LIDFa and LIDFb).

We selected several representative measurements from reflectance and SIF images to performance the retrieval as the NO method is computationally demanding. The average of reflectance or SIF within a randomly selected patch consisting of $5 \times 5$ pixels was taken as one measurement of a crop. We sampled 16 patches for every crop except wheat, for which only 8 patches were chosen due to the small size of the wheat field.

\subsection{Separation of canopy structure and plant physiology effects on SIF}

Fluorescence emission efficiency $\left(\epsilon_{F}\right)$ and radiative transfer factor $\left(\Gamma_{r t}\right)$ of the vegetation canopies represent the plant physiological contribution and canopy structural contribution to TOC SIF, respectively. Both PPAR and the scattering of SIF $\left(\sigma_{F}\right)$, and thus $\Gamma_{r t}$ are determined by vegetation canopy structure (including leaf properties), while $\epsilon_{F}$ is regulated by photosynthetic mechanisms. The fluorescence emission efficiency is especially useful for picking up variations in the 'condition of the canopy', in relation to stresses, and relating these variations to the capacity of the canopy photosynthesis. 
We simulated canopy structural contribution to TOC SIF by using the SCOPE model. The leaf parameters and canopy structure retrieved from TOC reflectance were sufficient to calculate the quantity $\Gamma_{r t}$. We first simulated TOC SIF with from RTMf for a given fluorescence emission efficiency $\left(\epsilon_{F}\right)$ and incident PAR, and then obtained $\Gamma_{r t}$ by normalizing the simulated SIF by this efficiency and incident PAR according to Eq. 2.1. The values used for the efficiency was 0.01 and for the incident PAR $250 \mathrm{~W} \mathrm{~m}^{-2}$. The efficiencies at $687 \mathrm{~nm}$ and $760 \mathrm{~nm}$ were $1.76 \times 10^{-4} \mathrm{~nm}^{-1}$ and $5.03 \times 10^{-5}$ $\mathrm{nm}^{-1}$, respectively. The values were not relevant to the simulation of canopy structural contribution since they cancelled out in the normalization. The fluorescence emission efficiency of the crops was finally obtained by removing the canopy structural contribution from TOC SIF, by dividing measured SIF by $\Gamma_{r t}$ (Eq. 2.1). The obtained value of $\epsilon_{F}$ is an effective value for the whole canopy.

\subsection{Model sensitivity and error propagation}

The Jacobians of SCOPE for reflectance and $\Gamma_{r t}$ were computed to (1) understand the relationships between the measured reflectance and the vegetation variables and $\Gamma_{r t}$; (2) identify parameters of the model that cause significant variety in reflectance and in $\Gamma_{r t} ;(3)$ assess the ill-posedness in estimation of $\Gamma_{r t}$. Jacobians $\left(\mathbf{J}_{R}\right.$ and $\left.\mathbf{J}_{\Gamma}\right)$ were computed for each set of optimized parameters and for each crop as:

$$
\begin{gathered}
\mathbf{J}_{R}=\left[\begin{array}{ccc}
\partial R_{\lambda_{1}} / \partial p_{1} & \ldots & \partial R_{\lambda_{1}} / \partial p_{n} \\
\vdots \ldots & \ddots & \vdots \\
\partial R_{\lambda_{b}} / \partial p_{1} & \ldots & \partial R_{\lambda_{b}} / \partial p_{n}
\end{array}\right] \\
\mathbf{J}_{\Gamma}=\left[\begin{array}{ccc}
\partial \Gamma_{640} / \partial p_{1} & \ldots & \partial \Gamma_{640} / \partial p_{n} \\
\vdots \ldots & \ddots & \vdots \\
\partial \Gamma_{850} / \partial p_{1} & \ldots & \partial \Gamma_{850} / \partial p_{n}
\end{array}\right]
\end{gathered}
$$

where $\mathbf{J}_{R}$ is the local model sensitivity of reflectance at each wavelength of the $b$ bands of the HyPlant DUAL module $(b=623)$ to each of the 10 optimized parameters, and $\mathbf{J}_{\Gamma}$ is the local model sensitivity of $\Gamma_{r t}$ spectra at each fluorescence wavelength (i.e. $640 \mathrm{~nm}-850 \mathrm{~nm}, 1 \mathrm{~nm}$ resolution) to each 
parameter. In total, we had 56 reflectance (i.e. 16 for rapeseed, barley and corn, and 8 for wheat) spectra on each day, and thus 56 sets of optimized parameters and Jacobians. We analysed the Jacobians for measurements on day 1 only, because the Jacobians on day 2 were very similar to those on day 1 .

We estimated the propagation of noise in the measured reflectance spectra to the uncertainty (i.e., the standard deviation) in model parameters. The model is described with a locally linear approximation by $\Delta \mathbf{R}=\mathbf{J}_{R} \Delta \mathbf{p}$, where $\mathbf{p}$ is a matrix of the model parameters and $\mathbf{R}$ is reflectance spectra. Because $\mathbf{J}_{R}$ is not a square matrix, it does not have an inverse. The inverse model is

$$
\Delta \mathbf{p}=\left(\mathbf{J}_{R}^{\mathrm{T}} \mathbf{J}_{R}\right)^{-1} \mathbf{J}_{R}^{\mathrm{T}} \Delta \mathbf{R}
$$

Therefore, a covariance matrix of $\mathbf{p}$ as a result of noise in can be obtained as:

$$
\begin{aligned}
E\left(\Delta \mathbf{p} \Delta \mathbf{p}^{\mathrm{T}}\right) & =E\left[\left(\mathbf{J}_{r}^{\mathrm{T}} \mathbf{J}_{R}\right)^{-1} \mathbf{J}_{R}^{\mathrm{T}} \Delta \mathbf{R} \Delta \mathbf{R}^{\mathrm{T}} \mathbf{J}_{R}\left(\mathbf{J}_{R}^{\mathrm{T}} \mathbf{J}_{R}\right)^{-1}\right] \\
& =\left(\mathbf{J}_{R}^{\mathrm{T}} \mathbf{J}_{R}\right)^{-1} \mathbf{J}_{R}^{\mathrm{T}} E\left(\Delta \mathbf{R} \Delta \mathbf{R}^{\mathrm{T}}\right) \mathbf{J}_{R}\left(\mathbf{J}_{R}^{\mathrm{T}} \mathbf{J}_{R}\right)^{-1}
\end{aligned}
$$

where $E\left(\Delta \mathbf{R} \Delta \mathbf{R}^{\mathrm{T}}\right)$ is the covariance matrix of the reflectance measurements. We assumed this covariance matrix is diagonal and uniform (all diagonal elements equal), characterized by a variance $\sigma_{R}^{2}$, and compuated the covariance matrix of the retrieved parameters due to measurement noise as:

$$
E\left(\Delta \mathbf{p} \Delta \mathbf{p}^{\mathrm{T}}\right)=\left(\mathbf{J}_{R}^{\mathrm{T}} \mathbf{J}_{R}\right)^{-1} \sigma_{R}^{2}
$$

For the noise of reflectance measurements $\sigma_{R}$ we used the spectrally averaged standard deviation of reflectance measurements of each reference panel (black, grey and white) (see Fig. S2 in the supplementary materials) $\left(\sigma_{R}=0.012\right)$. This uncertainty was comparable to the averaged change of reflectance of these panels in the two days, which was 0.014 . This provides reflectance uncertainty due to instrumental noise, but it does not represent the total measurement uncertainty (Van der Tol et al., 2016) 
We further estimated the propagation of uncertainty in the retrieved parameters caused by noise in reflectance into model simulation of $\Gamma_{r t}$.

$$
\begin{aligned}
E\left(\Delta \boldsymbol{\Gamma} \Delta \boldsymbol{\Gamma}^{\mathrm{T}}\right) & =E\left(\mathbf{J}_{\Gamma} \Delta \mathbf{p} \Delta \mathbf{p}^{\mathrm{T}} \mathbf{J}_{\Gamma}^{\mathrm{T}}\right)=\mathbf{J}_{\Gamma} E\left(\Delta \mathbf{p} \Delta \mathbf{p}^{\mathrm{T}}\right) \mathbf{J}_{\Gamma}^{\mathrm{T}} \\
& =\mathbf{J}_{\Gamma}\left(\mathbf{J}_{R}^{\mathrm{T}} \mathbf{J}_{R}\right)^{-1} \mathbf{J}_{\Gamma}^{\mathrm{T}} \sigma_{R}^{2}
\end{aligned}
$$

The standard deviation (uncertainty) of the simulated radiative transfer factor $\left(\sigma_{\Gamma}\right)$ were then found as the square roots of the diagonal elements of this matrix $\left(E\left(\Delta \boldsymbol{\Gamma} \Delta \boldsymbol{\Gamma}^{\mathrm{T}}\right)\right)$.

\subsection{Simulation of photosynthetic response to high temperature}

We simulated the photosynthetic response of the crops to high temperature by using a biochemical model as a validation of our retrieved $\epsilon_{F}$ on the two days.

We modelled the photosynthetic efficiency $\left(\epsilon_{P}\right)$, fluorescence emission efficiency $\left(\epsilon_{F}\right)$ and heat dissipation efficiency $\left(\epsilon_{H}\right)$ for both C3 and C4 plants. Of the investigated crops, corn has $\mathrm{C} 4$ photosynthetic pathway and the other three crops have C3 pathway. C3 and C4 plants have different photosynthetic regulation mechanisms, which are considered and implemented in the biochemical model. For the simulations, we used standard model input: $V_{\text {cmax }}=30 \mu \mathrm{mol} \mathrm{m}{ }^{-2} \mathrm{~s}^{-1}$ and $70 \mu \mathrm{mol} \mathrm{m}{ }^{-2} \mathrm{~s}^{-1}$ for $\mathrm{C} 3$ and $\mathrm{C} 4$ plants respectively, based on tabled values of Wullschleger (1993). The absorbed PAR by chlorophyll $\left(\mathrm{APAR}_{c h l}\right)$ was roughly approximated based upon the incident PAR, and was set to $250 \mathrm{~W} \mathrm{~m}^{-2}$. The leaf temperature was set from $10^{\circ} \mathrm{C}$ to $40^{\circ} \mathrm{C}$ with a step of $1^{\circ} \mathrm{C}$.

\section{Results}

\subsection{Mapping the effects of the heat wave on SIF}

Temperature of the canopies significantly increased during the heat wave (2 July 2015, 'day 2') compared with the temperature shortly before the heat wave (30 Jun 2015, 'day 1') (Fig. 2.5 and Table. 2.3). On day 1, corn had the highest temperature (average temperature was $33.0^{\circ} \mathrm{C}$ ) during the flight. However, it increased the least $\left(3.7^{\circ} \mathrm{C}\right)$ among the four crops 
when the air temperature changed from $27^{\circ} \mathrm{C}$ to $34^{\circ} \mathrm{C}$. Barley and wheat had increases more than $6^{\circ} \mathrm{C}$, which was very close to the change of the air temperature $\left(7^{\circ} \mathrm{C}\right)$. Comparing air temperature with canopy temperature, Table. 2.3 shows that the canopy temperature $\left(T_{c}\right)$ of the four crops was all higher than the air temperature $\left(T_{a}\right)$ on both days from $2.1^{\circ} \mathrm{C}$ to $6.4{ }^{\circ} \mathrm{C}$. The variability of $T_{c}-T_{a}$ on the two days $\left(3.4^{\circ} \mathrm{C}\right)$ was highest in corn among the four crops.

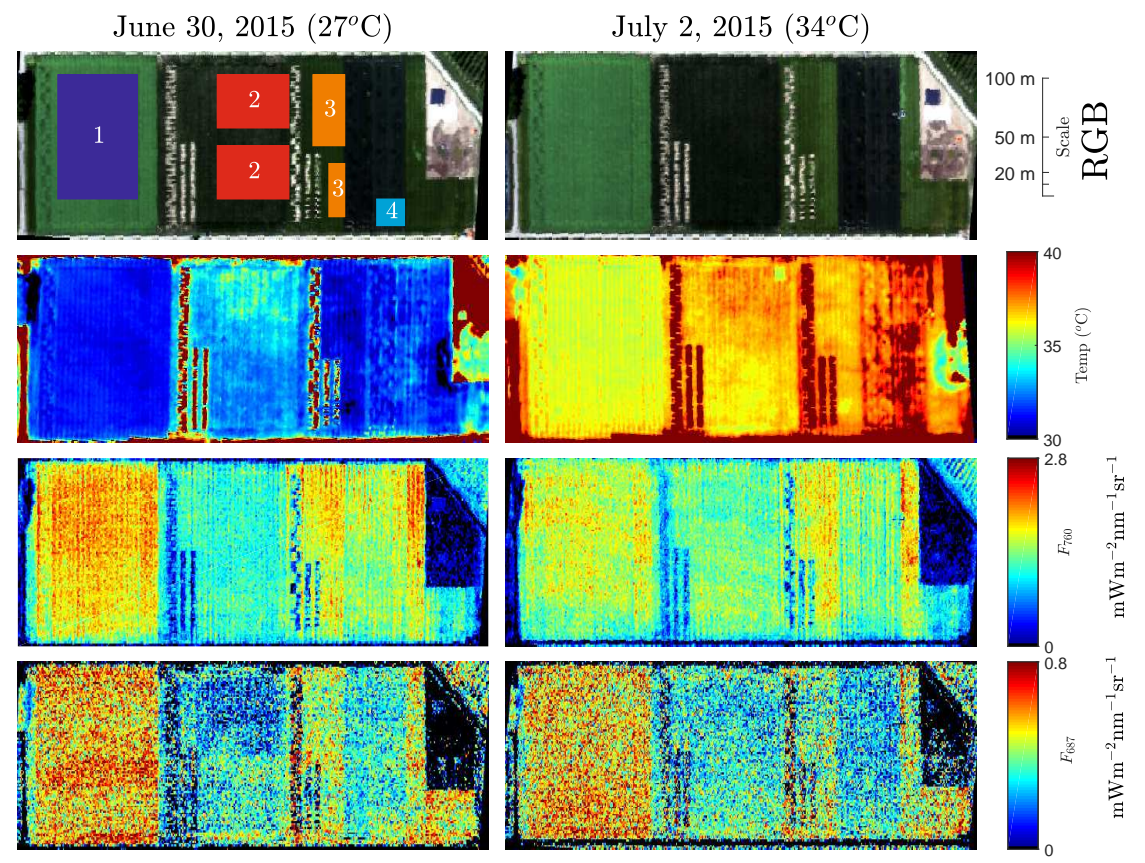

Figure 2.5: RGB, temperature, SIF at $687 \mathrm{~nm} F_{687}$ and at $760 \mathrm{~nm} F_{760}$ images of the experiment area before and during the heat wave. Crops are marked in the RGB image: 1: rapeseed; 2: corn; 3: barley; 4: wheat.

Fig. 2.5 and 2.6 show that TOC SIF responded to the heat event differently for the four crops. Rapeseed, wheat, and barley showed decreased SIF values while SIF in corn increased. Red SIF $\left(F_{687}\right)$ decreased by about $4.2 \%$ in rapeseed, $19.9 \%$ in barley and $10.9 \%$ in wheat. In contrast, in corn $F_{687}$ increased by about $18.0 \%$. Far red $\operatorname{SIF}\left(F_{760}\right)$ decreased by about $16.0 \%$, $13.7 \%$ and $2 \%$ for rapeseed, barley and wheat, respectively. Again, corn showed an increase of $17.0 \%$. Both the decrease in rapeseed, barley and 
Table 2.3: Temperature of the canopies $\left(T_{c}\right)$ on day 1 and on day 2 , and difference in canopy and air temperature $\left(T_{c}-T_{a}\right)$.

\begin{tabular}{lcccc}
\hline Temperature $\left({ }^{\circ} \mathrm{C}\right)$ & rapeseed & corn & barley & wheat \\
\hline$T_{c}$ (day 1) & $31.2 \pm 0.2$ & $33.0 \pm 0.7$ & $30.7 \pm 0.3$ & $31.8 \pm 0.4$ \\
$T_{c}$ (day 2) & $35.8 \pm 0.2$ & $36.7 \pm 0.5$ & $36.8 \pm 0.3$ & $38.1 \pm 0.6$ \\
$\Delta T_{c}$ & $4.6 \pm 0.3$ & $3.7 \pm 0.7$ & $6.1 \pm 0.4$ & $6.3 \pm 0.6$ \\
$T_{c}-T_{a}$ (day 1) & 4.6 & 6.4 & 4.1 & 5.2 \\
$T_{c}-T_{a}$ (day 2) & 2.1 & 3.0 & 3.1 & 4.4 \\
\hline
\end{tabular}

wheat, and the increase in corn were statistically significant ( $p$-value $<0.001)$, although $F_{687}$ and $F_{760}$ were broadly scattered. Comparing SIF at the two wavelengths, $F_{760}$ was much higher than $F_{687}$, and there was the slightly higher pixel variation in $F_{687}$. SIF values of bare soil pixels and reference panels were minimal compared to the values of vegetative pixels.

\subsection{Effects of extreme temperature on canopy reflectance, NDVI and PRI}

Reflectance spectra of crops taken before and during the heat wave differently changed across crops (Fig. 2.7). In the all visible region, rapeseed, wheat and barley showed an increased reflectance by $0.01-0.04$ (relatively $10 \%-104 \%)$. Corn showed a different behaviour than the other three crops: its visible reflectance slightly decreased during the heat wave by around $5 \%$, where greatest decreases happened in the red spectrum (Fig. 2.7H) reaching $21.4 \%$ at $670 \mathrm{~nm}$ (i.e. the region of red chlorophyll absorption). This decrease of red reflectance was most likely related to the ongoing canopy growth and increase of LAI in corn, thus corn continued to grow despite the heat event. Near infrared (NIR) reflectance decreased in rapeseed and wheat. It increased by $4.2 \%$ in corn and $7.5 \%$ in barley. Short-wave infrared (SWIR) reflectance increased in rapeseed, wheat and barley by $12.6 \%, 13.0 \% 30.7 \%$, respectively, but decreased by $7.4 \%$ in corn (Fig. 2.7D).

NDVI changed less than 0.04 for the four crops (Fig. 2.8A). It increased by $5 \%$ in corn and decreased in barley by $8 \%$. The change of the average PRI in barley, wheat and rapeseed was all less than 0.01 while the change in corn was more than 0.03 . PRI of the corn canopy was much higher on day 2 

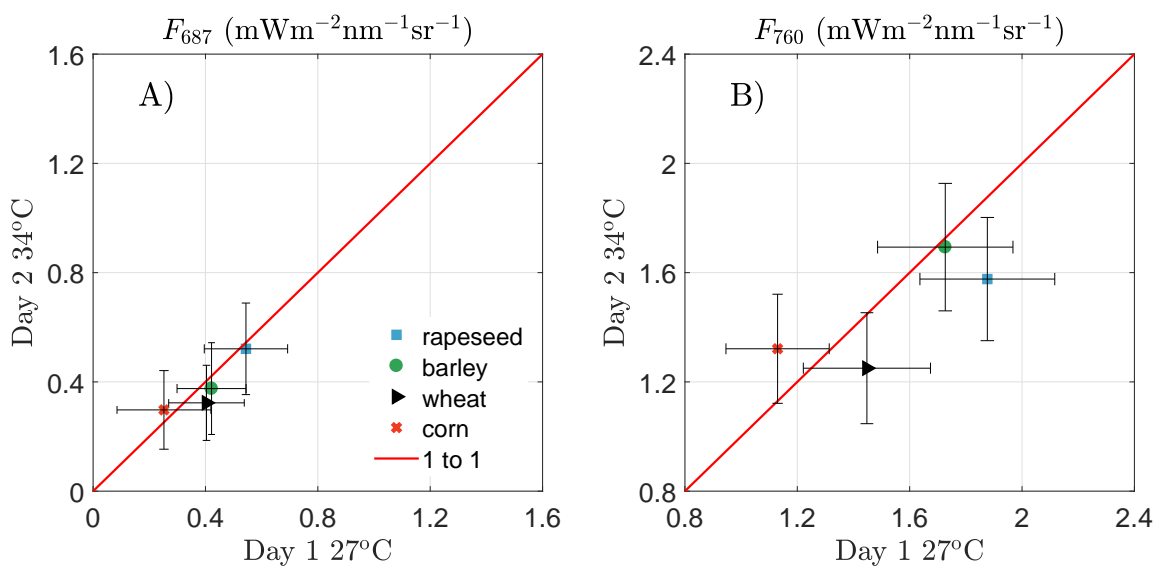

Figure 2.6: Red fluorescence $\left(F_{687}\right)$ and far red fluorescence $\left(F_{760}\right)$ on day 1 (before the heat wave) and day 2 (during the heat wave). The horizontal and vertical error bars represent the standard deviation of measurements at day 1 and day 2 , respectively.

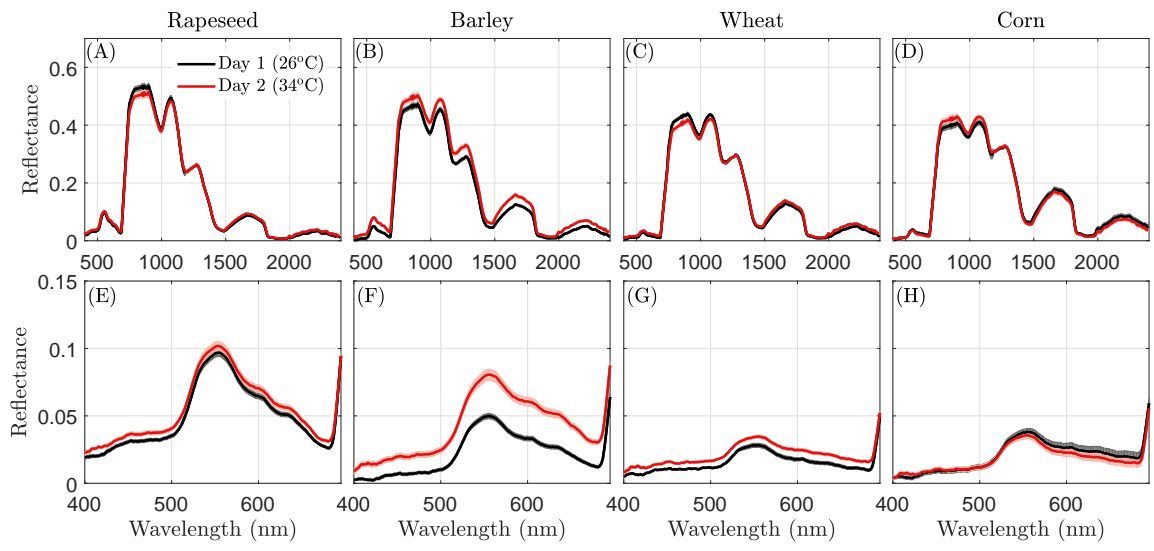

Figure 2.7: Reflectance measurements before (day 1) and during (day 2) the heat wave in the spectral region of $400-2500 \mathrm{~nm}$ and of $400-700 \mathrm{~nm}$. The buffers represent the standard deviation of the measurements.

than on day 1 while that of barley and wheat was lower on day 2 (Fig. 2.8B).

There was a slight increase of PRI of rapeseed. 

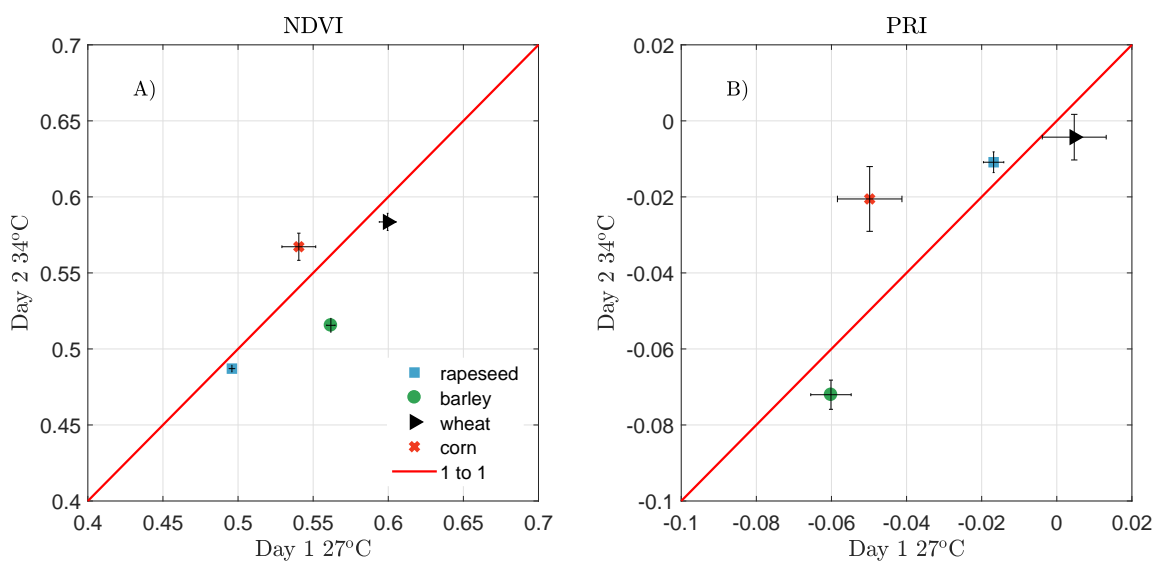

Figure 2.8: Normalized difference vegetation index (NDVI) and photochemical reflectance index (PRI) before (day 1) and during (day 2) the heat wave. The horizontal and vertical error bars represent the standard deviation of measurements at day 1 and day 2 , respectively.

\subsection{Parameter retrievals from reflectance spectra}

Fig. 2.9 and 2.10 show the simulated (fitted) reflectance of the crops before and during the heat wave, along with the measured reflectance. In general, the model reproduced the measured TOC reflectance well (RMSE between 0.006 and 0.01 ). The residuals were within \pm 0.02 , except in the spectral regions of 1400 - $1500 \mathrm{~nm}$ and 1800 - $1900 \mathrm{~nm}$ (i.e., water absorption bands), where spectral shifts in surface reflectance between the model simulations and field measurements were found. The discrepancy could be caused by the atmospheric correction, or by a limitation of the model representation. Compared to the standard deviation of the measurements of reflectance (in the 16 or 8 patches), the residuals were lower except for several spectral regions. 


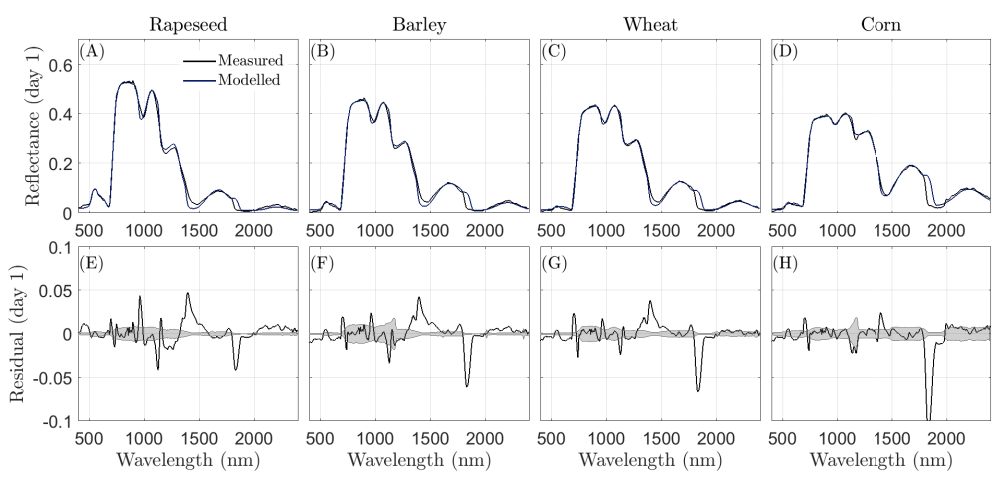

Figure 2.9: Upper panels: Measured (blue) and modelled (black) reflectance spectra of the four crops before the heat wave (day 1). Lower panels: the residual after spectral fitting (line) (i.e., the difference between measured and simulated apparent reflectance), and the standard deviation of the measurements (grey area).

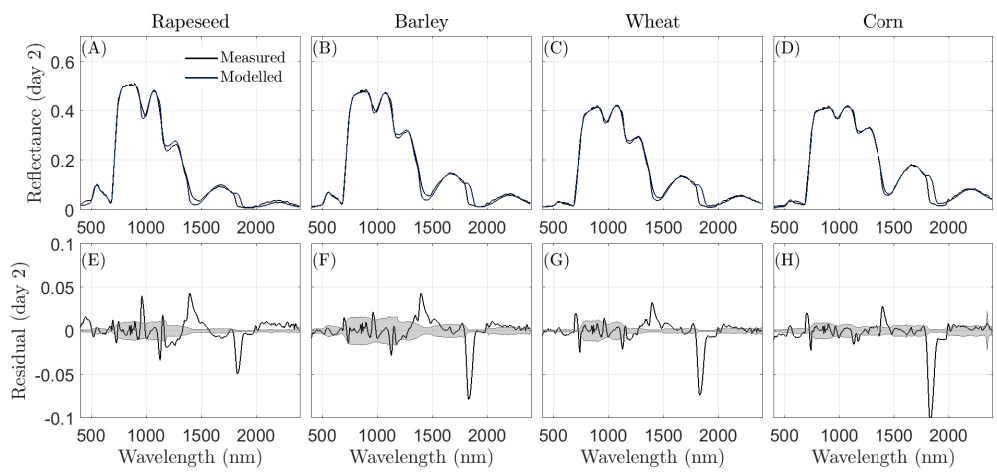

Figure 2.10: Upper panels: Measured (black) and modelled (blue) reflectance spectra of the four crops during the heat wave (day 2). Lower panels: the residual after spectral fitting (line) (i.e., the difference between measured and simulated apparent reflectance), and the standard deviation of the measurements (grey area).

Table 2.4 lists the retrieved key parameter values. The use of model inversion allows retrieving the whole reflectance spectra (rather than few bands used to calculate indices) to vegetation properties. In the two days, LAI changed less than 0.1. Both soil moisture $\left(S M_{p}\right)$ and leaf water content decreased $\left(C_{w}\right)$ in rapeseed, barley and wheat, while in the corn canopy they slightly increased. Leaf chlorophyll content decreased in rapeseed, barley and wheat, while increased in corn. Again, this decrease of chlorophyll was 
Table 2.4: The retrieved values of soil moisture $\left(S M_{p}\right)$ leaf chlorophyll content $\left(C_{a b}\right)$, leaf water content $\left(C_{w}\right)$, canopy LAI and canopy average leaf angle (ALA) before and during the heat wave.

\begin{tabular}{lcccccccc}
\hline Parameter & \multicolumn{2}{c}{ Rapeseed } & \multicolumn{2}{c}{ Barley } & \multicolumn{2}{c}{ Wheat } & \multicolumn{2}{c}{ Corn } \\
& day 1 & day 2 & day 1 & day 2 & day 1 & day 2 & day 1 & day 2 \\
\hline$S M_{p}(\%)$ & 14.6 & 12.5 & 14.0 & 10.8 & 14.0 & 9.4 & 6.7 & 9.4 \\
$C_{a b}\left(\mu \mathrm{g} \mathrm{cm}^{-2}\right)$ & 22.9 & 21.7 & 32.6 & 27.4 & 50.2 & 49.4 & 41.0 & 48.9 \\
$C_{w}\left(\mathrm{~cm}^{-1}\right)$ & 0.070 & 0.065 & 0.040 & 0.036 & 0.038 & 0.036 & 0.022 & 0.027 \\
$\mathrm{LAI}$ & 4.1 & 4.0 & 4.3 & 4.3 & 3.7 & 3.6 & 2.9 & 3.0 \\
$\mathrm{ALA}\left({ }^{\circ}\right)$ & 51.6 & 58.0 & 64.8 & 67.0 & 66.3 & 69.1 & 70.9 & 66.1 \\
$\mathrm{RMSE}$ of refl & 0.01 & 0.01 & 0.008 & 0.006 & 0.007 & 0.006 & 0.007 & 0.006 \\
\hline
\end{tabular}

consistent with the decrease of red reflectance in corn, and was most likely related to the ongoing canopy growth. Changes in retrieved LIDFa revealed that ALA of rapeseed, barley and wheat increased $2.2^{\circ}-6.4^{\circ}$, but it reduced from $70.9^{\circ}$ to $66.1^{\circ}$ in the corn canopy.

\subsection{Canopy structure and plant physiology effects on SIF}

Fig. 2.11 shows canopy structural contribution to TOC SIF (radiative transfer factor of SIF, $\Gamma_{r t}$ ) estimated from SCOPE, provided leaf optical properties and canopy structure parameters before and during the heat event. The radiative transfer factor was spectrally dependent and the spectra were similar to vegetation reflectance: lower values in the red region and much higher values in the NIR region. It increased in barley, wheat and corn, and the increase was much more significant in the NIR region than in the visible region. The changes of canopy structural contribution to TOC SIF $\left(\Delta \Gamma_{r t}\right)$ in barley, wheat and corn were significant compared to the uncertainty in the model prediction $\left(\sigma_{\Gamma}\right)$ caused by the reflectance measurements noise $\left(\sigma_{R}\right)$. The change in rapeseed was in the same magnitude of uncertainty.

Fig. 2.12 shows physiological contribution to TOC SIF in form of fluorescence emission efficiency $\left(\epsilon_{F}\right)$ estimated from measured $F_{687}$ and $F_{760}$ Despite red SIF was much lower than far red SIF for every crop, the retrieved efficiency at $687 \mathrm{~nm}\left(\epsilon_{F 687}\right)$ was much higher than that at $760 \mathrm{~nm}\left(\epsilon_{F 760}\right)$. The former ranged from $1 \times 10^{-4}$ to $5 \times 10^{-4} \mathrm{~nm}^{-1}$, while $\epsilon_{F 760}$ ranged from $5 \times 10^{-5}$ to $1 \times 10^{-4} \mathrm{~nm}^{-1}$. The ratio between $\epsilon_{F 687}$ and $\epsilon_{F 760}$ ranged from 

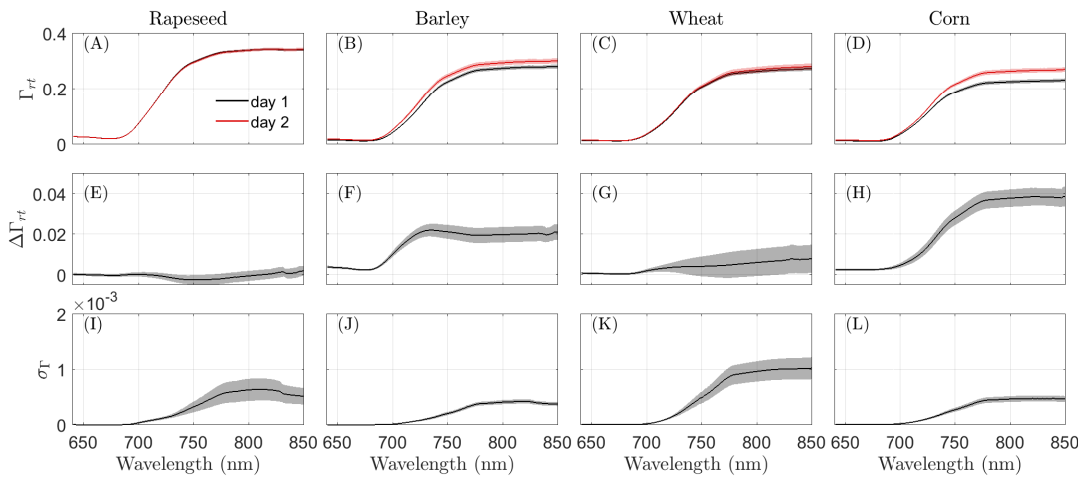

Figure 2.11: Upper panels: canopy structural contribution to TOC SIF (radiative transfer factor of SIF, $\Gamma_{r t}$ ) estimated from SCOPE before (day 1 ) and during (day 2) the heat wave of the four crops. Middle panels: the difference of $\Gamma_{r t}$ before and during the heat wave $\left(\Delta \Gamma_{r t}\right)$. Lower panels: the uncertainty of $\Gamma_{r t}\left(\sigma_{\Gamma}\right)$ caused by the uncertainty in the reflectance measurements. The buffers represent the standard deviations of the 16 or 8 patches.

2.8 to 3.8 for all the pixels on day 1 and day 2 . This ratio was close to the ratio used in SCOPE, which is 3.6 (i.e. $1.76 \times 10^{-4} \mathrm{~nm}^{-1} / 5.03 \times 10^{-5} \mathrm{~nm}^{-1}$ ).

The efficiency of rapeseed, barley and wheat decreased at both wavelengths, while it slightly increased in corn.
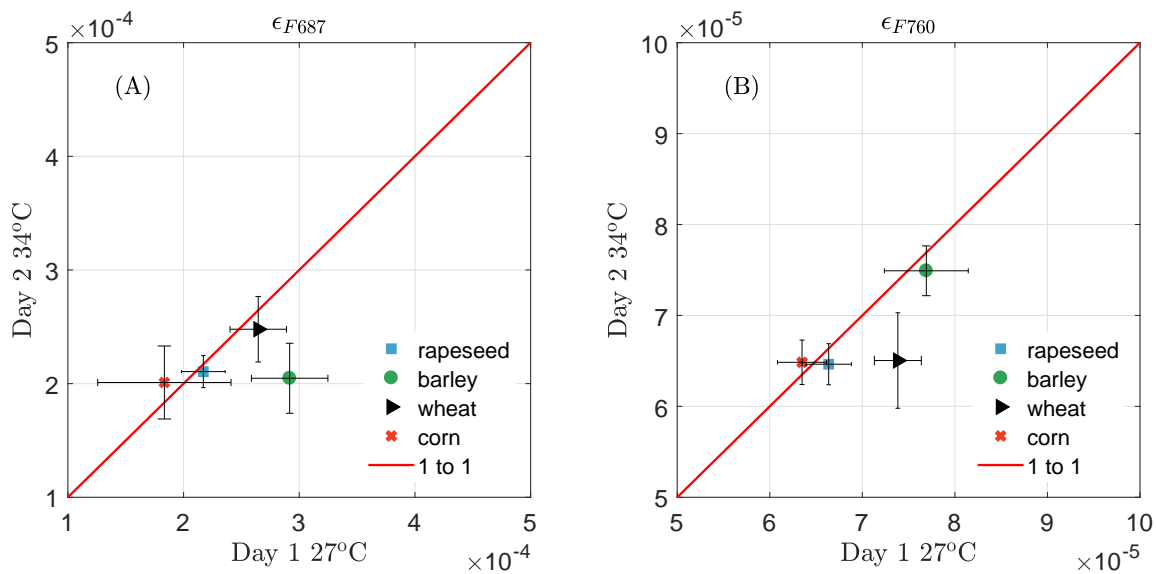

Figure 2.12: Retrieved values of fluorescence emission efficiency (i.e. photosynthetically determinative factor of the TOC SIF) at $687 \mathrm{~nm}\left(\epsilon_{F 687}\right)$ and 760 $\mathrm{nm}\left(\epsilon_{F 760}\right)$ before and during the heat wave of the four crops. The horizontal and vertical error bars represent the standard deviation of measurements at day 1 and day 2 , respectively. 
Table. 2.5 shows the changes in measured fluorescence, estimated $\Gamma_{r t}$ and $\epsilon_{F}$ before and during the heat wave. $\epsilon_{F}$ estimated from $F_{687}$ and $F_{760}$ each increased about $9.6 \%$ and $2.1 \%$ respectively in the corn canopy but decreased in the the other three crops (i.e. ranging from $2.6 \%$ to $29.8 \%$ ).

Table 2.5: Relative changes (\%) in TOC SIF measurements at $687 \mathrm{~nm}$ and $760 \mathrm{~nm}\left(F_{687}\right.$ and $\left.F_{760}\right)$, in fluorescence emission efficiency $\left(\epsilon_{F}\right)$ retrieved from $F_{687}$ and $F_{760}$, in radiative transfer factor of SIF $\left(\Gamma_{r t}\right)$ at these two wavelengths before and during the heat wave for the crops. The sign '-' indicates a decrease on day 2 (during the heat wave).

\begin{tabular}{lcccccc}
\hline \multirow{2}{*}{ Changes (\%) } & \multicolumn{2}{c}{$\Gamma_{r t}$} & \multicolumn{2}{c}{$\epsilon_{F}$} & \multicolumn{2}{c}{ TOC SIF } \\
& $687 \mathrm{~nm}$ & $760 \mathrm{~nm}$ & $687 \mathrm{~nm}$ & $760 \mathrm{~nm}$ & $687 \mathrm{~nm}$ & $760 \mathrm{~nm}$ \\
\hline Rapeseed & -0.4 & -0.7 & -3.0 & -2.6 & -4.2 & -16.0 \\
Barley & 23.1 & 8.3 & -29.8 & -2.6 & -19.9 & -13.7 \\
Wheat & 3.9 & 1.9 & -6.3 & -11.9 & -10.9 & -2.0 \\
Corn & 20.7 & 15.6 & 9.6 & 2.1 & 18.0 & 17.0 \\
\hline
\end{tabular}

Fig.2.13 shows that vegetation canopy structure, physiology and incident light all significantly contributed to observed changes in measured SIF. The changes in canopy structure including leaf angle, sun position, leaf pigments and leaf water content accounted for $8 \%$ to $58 \%$ of changes in measured SIF. Differences of SIF values between both days caused by incident light intensity were about $12 \%$ to $64 \%$. Eventually, $20 \%$ to $56 \%$ of the observed variation in TOC SIF was caused by the photosynthetic regulation. Most change of TOC SIF in corn was induced by the change of incident light intensity. The canopy structure contributed less than $20 \%$ of the variation of TOC SIF. The canopy structure had significant effects on change of SIF in rapeseed.

\subsection{Modelling the impact of extreme temperature on photosynthetic light partitioning}

Fig. 2.14 shows the simulations from the biochemical model of Van der Tol et al. (2014). The efficiencies of both fluorescence emission, photosynthesis and heat dissipation responded to high temperature $\left(>32^{\circ} \mathrm{C}\right)$ differently for C3 and C4 crops: C3 plants reduced in both, while in C4 plants they slightly increased. The heat dissipation of C3 plants increased with leaf temperature 
$\left(>32^{\circ} \mathrm{C}\right)$, but it decreased in $\mathrm{C} 4$ plants. The maximum photosynthetic efficiency occurred when the leaf temperature was $28^{\circ} \mathrm{C}$ for $\mathrm{C} 3$ plants and was $35^{\circ} \mathrm{C}$ for $\mathrm{C} 4$ plants.
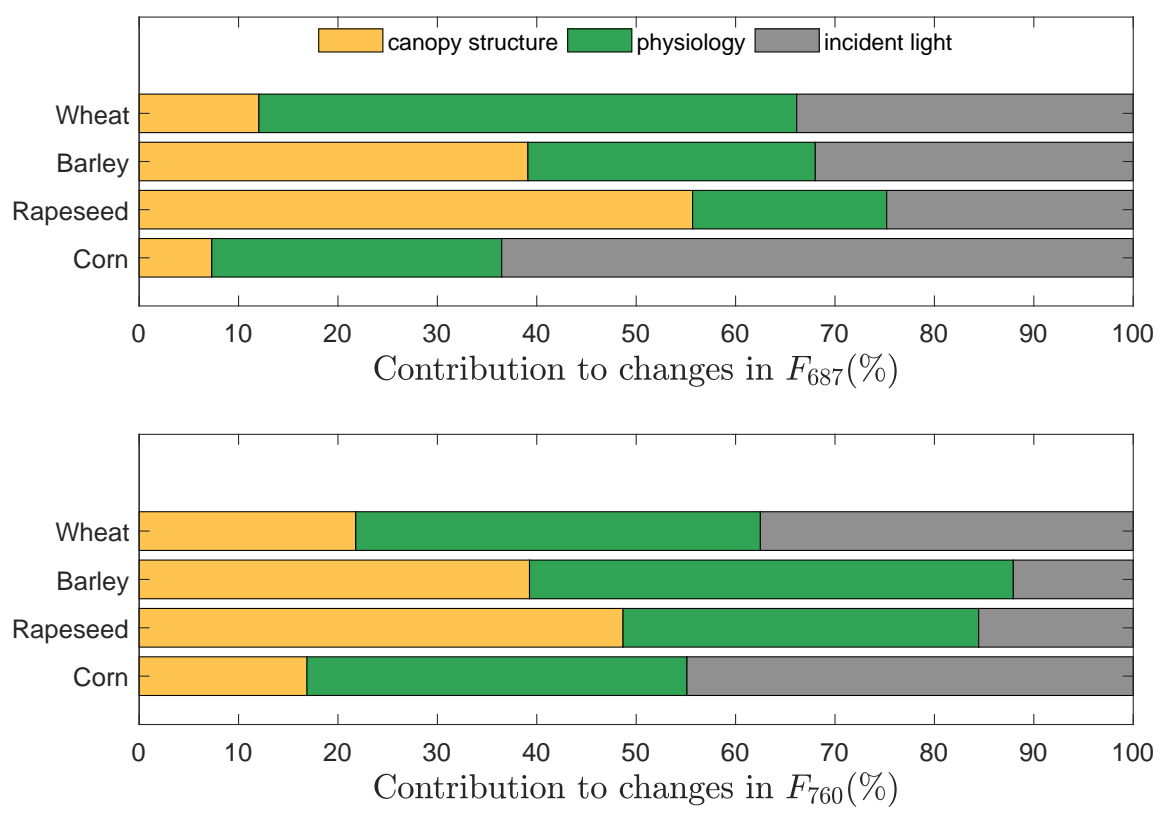

Figure 2.13: Relative contribution of canopy structure, plant physiology and incident light intensity to the changes in TOC SIF measurements $\left(F_{687}\right.$ and $\left.F_{760}\right)$ before and during the heat wave. 

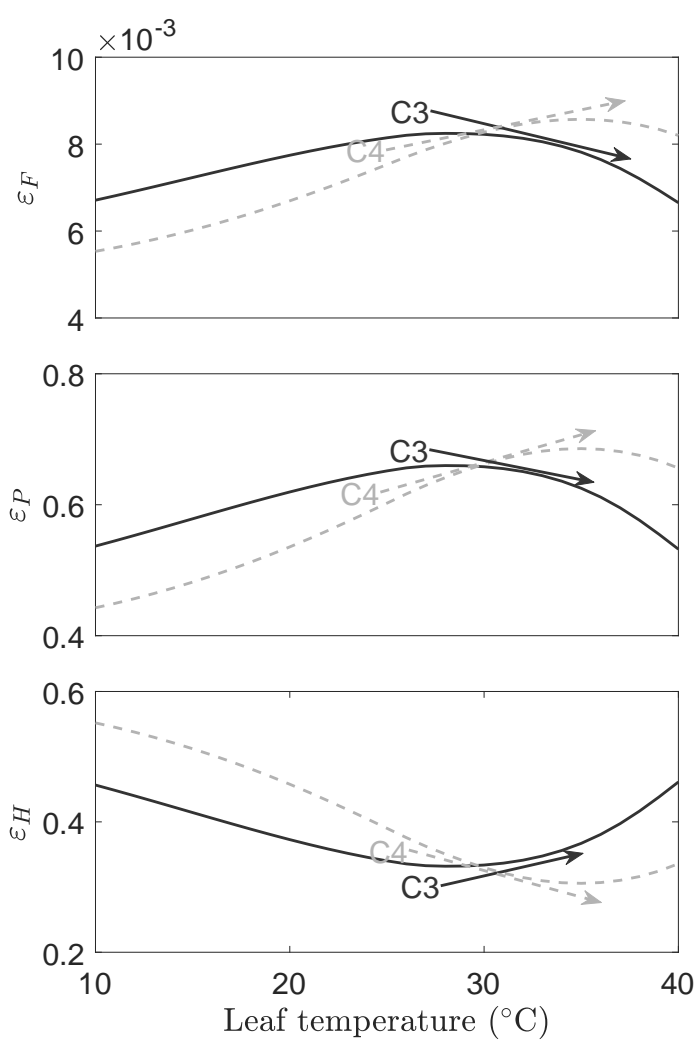

Figure 2.14: The efficiencies of three pathways of absorbed energy in photosystems changing with leaf temperature from 15 to $40{ }^{\circ} \mathrm{C}$ : fluorescence emission $\epsilon_{F}$, photochemistry $\epsilon_{P}$, and heat dissipation $\epsilon_{H}$.

\section{Discussion}

\subsection{Structural and physiological effects on SIF}

Most physiological stress regulating mechanisms in both photosystems will affect the fluorescence emission at photosystem level (Rascher et al., 2015), which can be measured with remote sensing techniques. However, TOC SIF signals are determined by canopy structure and incident light intensity besides of plant physiology (Eq. 2.1). Consequently, variations in TOC SIF cannot be directly interpreted as photosynthetic changes because of the contribution of canopy structure and incident light intensity, which are related to PAR absorption and SIF re-absorption.

The use of reflectance and RTMs provides a way to quantify and 
compensate for vegetation structural effects on TOC SIF $\left(\Gamma_{r t}\right)$, and thus obtain the efficiency of fluorescence $\left(\epsilon_{F}\right)$ at photosystem level, which is an indicator of the status of the light reactions of photosynthesis.

Despite the ill-posedness in the retrieval of vegetation parameters from reflectance, estimates of canopy structural contribution to SIF $\left(\Gamma_{r t}\right)$ is reliably possible. What matters is the sensitivity of $\Gamma_{r t}$ to reflectance, and the uniqueness of the relationship between reflectance and $\Gamma_{r t}$, rather than the sensitivity of reflectance to the individual model parameters. Indeed some parameters have little influence on reflectance, and these parameters cannot be retrieved accurately. However, it appears that these parameters do not affect $\Gamma_{r t}$ either. For example, the accuracy in retrieval of leaf properties $C_{s}$ and $C_{c a}$ and canopy structure parameter LIDFb from reflectance is not guaranteed as TOC reflectance is not sufficiently sensitive to these parameters (Jacquemoud et al., 1995; Jacquemoud et al., 2009), but SIF is not sensitive to these parameters either (Verrelst et al., 2016; Van der Tol et al., 2016), and thus uncertainties of these parameters will have little effect on uncertainties in $\Gamma_{r t}$. In contrast, SIF is more sensitive to LAI, $C_{a b}$, average leaf angle; parameters for which retrievals are usually reliable (Jacquemoud et al., 1995; Weiss et al., 2004; Houborg et al., 2007; Darvishzadeh et al., 2008). It should be noted that even the retrieval of these parameters can be ill-posed. Van der Tol et al. (2016) showed that the effect of $C_{d m}$ and LAI on reflectance is complementary in the VNIR region. However, these parameters have a similar complementary effect on $\Gamma_{r t}$, and this further reduces the uncertainty in $\Gamma_{r t}$ Indeed, reflectance and $\Gamma_{r t}$ are quite similar in terms of spectral shape (Fig. 2.11): both scattering of $\operatorname{SIF}\left(\sigma_{F}\right)$ and reflectance are lower in the visible bands and higher in the near-infrared bands (Porcar Castell et al., 2014; Yang and van der Tol, 2018).

Further indication that the estimates of $\Gamma_{r t}$ are reliable are the realistic values of the resulting values for $\epsilon_{F}$ in the two $\mathrm{O}_{2}$ absorption bands. The ratio between $\epsilon_{F 687}$ and $\epsilon_{F 760}$ matches well with the prior ratio as used in SCOPE, which is calibrated to leaf-level fluorescence measurements. 


\subsection{Monitoring crops response to heat temperature}

The dataset used in this study is unique: It consists of simultaneously measured reflected radiance, fluorescence and land surface temperature shortly taken before and during a heat wave. The dataset and our approach discussed above allow monitoring the responses of crops to the heat wave.

In this study both $F_{687}$ and $F_{760}$ for rapeseed, barley and wheat decreased (Fig. 2.5 and 2.6), despite the higher irradiance on day 2 (i.e. more light available for fluorescence). This decrease of SIF has two causes: (1) the reduction of fluorescence emission efficiency (Fig. 2.12) and (2) a decrease in the fraction of absorbed photosynthetic active radiation (fPAR). The latter has been frequently reported during heat events (Jolly et al., 2005; Reichstein et al., 2007), and this was also the case in our study (as a result of changes in canopy structure).

The interpretation of the observations from the RTM promotes a better understanding of SIF signals. The physical, physiological, and methodological factors jointly control fluorescence (Porcar Castell et al., 2014; Damm et al., 2015b). Observed significant variation in TOC SIF measurements do not necessarily indicate significant changes in plant functional status (e.g. rapeseed, barley and corn at $760 \mathrm{~nm}$ in Table. 2.5). Reversely, changes in $\epsilon_{F}$ may not result in a change of measured SIF (wheat in Table. 2.5). The separation of structural and physiological factors provides insights into short-term responses crops to temperature extremes.

The changes in reflectance were converted into changes in canopy structure (e.g. ALA), and leaf properties (e.g. leaf water content) by using model inversion (Fig. 2.2). The increase of ALA in rapeseed, barley and wheat during the heat event is a protective leaf movement from excessive radiation. It matches with the earlier observation elsewhere that ALA increases during drought stressed soybeans (Biskup et al., 2007). The decrease of surface soil moisture and leaf water content in rapeseed, barley and wheat during the heat event is consistent with our expectation: no rain occurred between the days while the evaporative demand was high. The increase of soil moisture 
in corn seems unrealistic. Most likely the model was not able to effectively separate canopy water from soil moisture. The increase of leaf water content due to ongoing growth of corn was apparent in the canopy reflectance, but due to ill-posedness it may (partly) be attributed to soil moisture in the retrieval. Other limitations of the SCOPE model representation of the crops may also have played a role in this study. For example, the ear and awns development in barley that occurred during this time of the season is not considered in the model. The bright ears and long highly reflective awns accounted for the great change in barley's visible reflectance. The use of SCOPE in this kind of canopy may lead to uncertainties in interpretation of reflectance measurements.

More interestingly, we found that fluorescence emission efficiency $\left(\epsilon_{F}\right)$ reduced in rapeseed, barley and wheat while increased in corn. This complies with the simulations from the biochemical model (Fig. 2.14). During the heat event when the leaf temperature was higher than $32^{\circ} \mathrm{C}$, the $\mathrm{C} 3$ crops (rapeseed, barley and wheat) were presumably under stress as manifested by a significant reduction of (simulated) photosynthetic efficiency and retrieved fluorescence emission efficiency $\left(\epsilon_{F}\right)$. The simulations show a similar effect of temperature on fluorescence emission efficiency and photochemistry efficiency, and thus a decrease of $\epsilon_{F}$ directly indicates a decrease of $\epsilon_{P}$. The relationship between $\epsilon_{F}$ and $\epsilon_{P}$ is regulated by the third pathway of absorbed radiation, non-photochemical quenching $\operatorname{NPQ}\left(\epsilon_{H}\right)$. An independent remotely sensed measure of $\epsilon_{H}$ will provide a quantitative measurement of $\epsilon_{P}$ without the need to rely on model simulations.

PRI provides a potential way to estimate $\epsilon_{H}$, and further prospect for monitoring and quantifying photosynthetic functioning remotely (Garbulsky et al., 2011). PRI correlates with the short-term reversible xanthophyll pigment changes and with the efficiency of photosynthesis (Gamon et al., 1992). A low PRI indicates a pigment composition that is associated to a high partitioning of excess energy to heat dissipation (high $\epsilon_{H}$ ). The significant increase of PRI in corn implies a reduction in $\epsilon_{H}$, and a concurrent increase in $\epsilon_{P}$ and in $\epsilon_{F}$. This is consistent with the retrieved $\epsilon_{F}$ change in corn and with 
the model simulations. Although it is well known that a correlation between PRI and $\epsilon_{H}$ exists, the quantification of $\epsilon_{H}$ from PRI or other reflectance information is still not consolidated. This is an important line to follow for monitoring photosynthesis from space.

\subsection{Implications}

High temperatures during a heat wave affect the photosynthetic functions of plants by changing the rate of chemical reactions and functional organization (Berry and Bjorkman, 1980; Pastenes and Horton, 1996). The higher vapour pressure deficit associated with heat also affects the transpiration rate. These changes provoke plant responses to balance the investment in the resources of energy, water and carbon dioxide, and maintain co-limitated of photosynthesis by different resources (Bloom et al., 1985; Field et al., 1995; Goetz et al., 1999). Responses include pigment and structural changes such as chloroplast avoidance movement and changes in leaf inclination. The reduced light harvest and the dissipation of absorbed radiation as heat, helps to avoid potential damage caused by excess radiation (Björkman and Powles, 1984; Long et al., 1994; Kasahara et al., 2002; Zygielbaum et al., 2009)

It is well known that the optimum temperatures for a range of important physiological processes are different for $\mathrm{C} 3$ and $\mathrm{C} 4$ crops. In the $\mathrm{C} 4$ pathway, dark and light reactions are separated, and the carboxylation takes place under a high $\mathrm{CO}_{2}$ concentration. This strongly suppresses photorespiration in $\mathrm{C} 4$ vegetation, resulting in a higher water use efficiency and lower sensitivity to heat and high vapour pressure deficit than $\mathrm{C} 3$ vegetation (Ehleringer and Björkman, 1977; Collatz et al., 1992). The fact that the increase of canopy temperature was the smallest in corn among the investigated crops (Table 2.3) indicates a high evaporation rate on both days (Jackson et al., 1981), and a limited effect of heat on stomatal aperture. This again is consistent with the increase in SIF and PRI in corn, and with the known resilience of vegetation of the $\mathrm{C} 4$ photosynthetic pathway to high temperature (Sage and Kubien, 2007; Yamori et al., 2014). It is worth noting that although we find clearly different responses of the three C3 crops (rapeseed, wheat and 
barley) and C4 crop (corn) to the heat wave, we are not certain that these are only caused by their different photosynthetic pathways because of other superimposing factors, such as different leaf age, different phenological stage and irrigation history (Berry and Bjorkman, 1980).

\section{Conclusion}

We demonstrate that different responses of vegetation to high temperature can be detected using the complementary information of hyperspectral reflectance and fluorescence. The combination of reflectance and SIF provides a comprehensive set of information to assess crop condition in presence of heat waves considering physiological, biochemical, and structural adaptations. The airborne HyPlant sensor allows for spatially representative, non-intrusive detection of these characteristics. This is relevant for determining the optimum growth conditions of crops and early stress detection. Solving confounding and superimposing effects of physiology biochemistry and structure on stress indicator retrieved from remote sensing data remains a challenge and requires further attention. 



\section{Linking canopy scattering of SIF with reflectance *}

*This chapter is based on: Yang, P., van der Tol, C., 2018. Linking canopy scattering of far-red sun-induced chlorophyll fluorescence with reflectance. Remote Sensing of Environment 209, 456 - 467. 


\section{Abstract}

Remotely sensed sun-induced chlorophyll fluorescence (SIF) has been used as an indicator of global terrestrial vegetation photosynthesis. The connection between SIF and photosynthesis allows its use for improving estimates of gross primary production (GPP) and monitoring plant stress. In these analyses, up-scaling of the relationship between SIF and photosynthesis from the photosynthetic level to the canopy, regional or global scale has been one of the main challenges. The scaling is strongly affected by the radiative transfer of emitted SIF, notably scattering and re-absorption of SIF. It is essential to understand these processes in order to differentiate effects of canopy structural variation from effects of photosynthesis functional variation on SIF. In this study, we derive the relationship between canopy scattering of SIF and top-of-canopy (TOC) reflectance analytically, by investigating the radiative transfer of incident light and emitted SIF. The similarity of radiative transfer of intercepted incident light and emitted SIF results in a simple relationship between reflectance and canopy scattering of SIF. In particular, we find that the ratio of far-red reflectance $(R)$ to the product of canopy interceptance $\left(i_{0}\right)$ and leaf albedo $(\omega)$ is an accurate estimate of canopy scattering of far-red SIF (i.e., $\sigma_{F C}=\frac{R}{i_{0} \omega}$ ). SCOPE model simulations are used to validate our findings. The relationship we found provides an easy and accurate approach for rapid decoupling canopy structural and functional regulation of SIF, and correction of SIF for bidirectional effects. This will improve estimates of canopy photosynthesis from SIF. 


\section{Introduction}

Sun-induced chlorophyll fluorescence (SIF) is a novel remote sensing signal for monitoring vegetation photosynthesis. It takes place in the pigment beds of photosystems, and SIF is an indicator of the efficiency by which photons are transmitted to photochemical reaction centers (Grace et al., 2007; Meroni et al., 2009). It is therefore closely related to the light harvesting process and responds timely to rapid changes in photosynthesis (Krause and Weis, 1991; Baker, 2008). In recent studies SIF was used to estimate vegetation photosynthetic capacity (Zhang et al., 2014) and for tracking dynamic changes of photosynthesis (Rossini et al., 2015). The connection between SIF and photosynthesis allows its use for improving the estimation of global or regional gross primary production (GPP) (Frankenberg et al., 2011; Guanter et al., 2014), and as an early warning signal of vegetation stress (Ač et al., 2015).

Apart from photosynthetic activity, SIF observations from remote sensing are strongly affected by the structure of vegetation canopies (Grace et al., 2007; Migliavacca et al., 2017; Damm et al., 2015a). SIF observed at top of canopy is only a portion of the total emitted SIF, due to re-absorption and scattering (i.e., they are complementary to each other) (Porcar Castell et al., 2014). The scattering and re-absorption of SIF from the moment of emission to the moment of escape from the canopy in observation direction, is (among other factors) sensitive to canopy leaf area index (LAI) and leaf orientation (Verrelst et al., 2015; Verrelst et al., 2016). Scattering and re-absorption effects are spectrally dependent. SIF at $760 \mathrm{~nm}$ (far-red SIF) is scattered more and re-absorbed less than SIF at $687 \mathrm{~nm}$ (red SIF) (Porcar Castell et al., 2014) and therefore the portion of SIF reaching the sensor is higher for far-red SIF than for red SIF. As a result, the ratio of red and far-red SIF from canopy observation differs from that of leaf-level measurements (Fournier et al., 2012; Cendrero-Mateo et al., 2015).

Understanding of canopy scattering of SIF is crucial, especially when a quantitative link between SIF and photosynthesis is desired for GPP estimates. 
In a regional SIF to GPP comparison, Guanter et al. (2014) assumed a canopy scattering coefficient of unity (i.e. no absorption) of far-red SIF due to lack of effective ways to quantify the scattering, but acknowledged the potential importance of accurate estimates of this process. Simulations with radiative transfer models (RTMs) confirmed that the scattering is an important aspect: They show that the relation between photosynthetic activity and SIF is canopy structure dependent (Damm et al., 2015a; Verrelst et al., 2016) and that a substantial portion of the variability of SIF at different spatial and temporal scales is due to canopy structure rather than photosynthetic functioning (Koffi et al., 2015; Van der Tol et al., 2016; Migliavacca et al., 2017).

One way to study the effect of the scattering is to compare leaf-level and canopy-level measurements. Cendrero-Mateo et al. (2015) found that topof-canopy (TOC) fluorescence of a wheat canopy measured in a growing season differed from leaf-average fluorescence, and that they developed differently over the growing season. The asymmetric evolution of leaf and canopy SIF may be attributed to seasonal changes in the scattering of fluorescence. Spectral differences between leaf and canopy SIF have also been reported. Fournier et al. (2012) reported that the ratio of red to far-red SIF of grass decreased by a factor of two from the leaf to the canopy level. This confirms that canopy scattering for the red and far-red SIF are different.

The empirical method (i.e. comparing leaf and canopy measurements) can reveal the magnitude of the effect of scattering in specific cases, but it is challenging to generalize the results. The need to sample a representative number of leaves that account for the variability of incident light and leaf properties makes the method labour intensive (Zarco-Tejada et al., 2003; Cendrero-Mateo et al., 2015). Furthermore, it is not easy to control the experiment and to identify and isolate the different effects on scattering, such as those of soil background, viewing and solar illumination angles.

RTMs offer a comprehensive complementary method to investigate the effects of canopy structure on TOC SIF. They provide an estimation of canopy scattering of SIF by simulating the light-canopy interaction. The 
SCOPE model (Van der Tol et al., 2009) simulates leaf fluorescence emission and TOC SIF, reflectance and photosynthesis of homogeneous canopies, while the mSCOPE model (Yang et al., 2017) simulates these for vertically heterogeneous canopies. For more complex canopies, 3D models have been developed, such as the DART (Gastellu-Etchegorry et al., 2017), FluorWPS (Zhao et al., 2016), and FluorFLIGHT (Hernández-Clemente et al., 2017). All these models require prior inputs of canopy structure and leaf properties. These are unknown in most remote sensing applications, but they can be retrieved by means of inverting a RTM using measured reflectance data (Houborg et al., 2007; Jacquemoud et al., 2009). The retrieved properties can then be used in a fluorescence RTM to quantify the SIF scattering. In this way, RTMs for reflectance and fluorescence can be used to interpret observed SIF signals.

Van der Tol et al. (2016) retrieved key biophysical and biochemical parameters from the reflectance data of rice canopies, and applied these parameters to simulate TOC SIF by using SCOPE. Such retrievals have a number of limitations: They are computational demanding. Both the retrieval of properties and prediction of SIF scattering are model dependent, and uncertainties in the estimation of canopy properties may be introduced due to ill-posed retrievals that may propagate into error in the prediction of canopy scattering of SIF. Nevertheless, Van der Tol et al. (2016) found that most of the variability of SIF could be reproduced after retrieval of parameters from reflectance. This not only confirms the dominating role of canopy scattering on seasonal variations of SIF, but also suggests that reflectance data can be used to estimate this scattering.

The idea that reflectance can explain the canopy scattering of SIF is promising. Reflectance data are widely available as many satellites have the capability to detect vegetation reflectance in many bands. The quantification of SIF scattering through reflectance measurements provides a way to decouple canopy structural and photosynthetic regulation effects on remotely sensed SIF.

Two recent studies provide experimental evidence of a close link 
between canopy scattering of SIF and reflectance. Badgley et al. (2017) reported that far-red reflectance times NDVI strongly correlates with SIF through the vegetated fraction of the surface. Liu et al. (2016) reported a bidirectional effect on SIF measurements that was similar to the effect on reflectance.

In the present study, we aim to link reflectance and canopy scattering of SIF by investigating the radiative transfer of incident light and emitted fluorescence with a minimum set of assumptions about the representation of canopies in models. We provide a detailed derivation of the canopy scattering of SIF and its relation to TOC reflectance. SCOPE model simulations are used to validate our findings. The relationship we found will allow rapid decoupling of canopy structural and functional regulation of SIF, which is useful for improving estimates of canopy photosynthesis from SIF.

\section{Theoretical basis}

\subsection{Definitions and aim of study}

The objective of the study is to relate the scattering of SIF $\left(\sigma_{F C}\right)$ to TOC (directional) reflectance $(R) . R$ and $\sigma_{F C}$ describe, respectively, the scattering of incident light and that of total emitted SIF to the viewing direction. They are defined as:

$$
R=\pi L_{o} / E
$$

$$
\sigma_{F C}=\pi L_{o}^{F} / E_{F}
$$

where $L_{o}$ and $L_{o}^{F}$ are the radiance of observed reflected solar radiation and of observed fluorescence at top of canopy, respectively, and $E$ and $E_{F}$ are the irradiance of incident flux at top of canopy and emitted fluorescence from all the leaves in the canopy (i.e., fluorescence emission), respectively. Note that an observation is always the sum of $L_{o}$ and $L_{o}^{F}$, and in remote sensing applications, they need to be separated with for example the FLD method 
(for a review, see Meroni et al., 2009). In radiative transfer modelling, they are separately simulated.

As mentioned, our objective is to express the canopy scattering of SIF $\sigma_{F C}$ as a function of reflectance $R$ :

$$
\sigma_{F C}=f(R)
$$

Reflectance and canopy scattering of SIF, and their relationship can be obtained if we know the observed radiance $\left(L_{o}\right)$, observed fluorescence radiance $\left(L_{o}^{F}\right)$, incident irradiance $(E)$ and canopy total fluorescence emission $\left(E_{F}\right)$. The incident irradiance is known in most cases either from measurements or from atmosphere radiative transfer models (e.g. MODTRAN (Berk et al., 2005)), but $E_{F}$ cannot be estimated easily.

In what follows, we derive an explicit expression of Eq. 3.3 in four steps, the final result of which is Eq. 3.12. First, we explain the interaction between incident flux and a vegetation canopy. Second, we provide an expression of canopy fluorescence emission. Third, we compare the equation for the observed reflected flux to that of the observed fluorescence flux. Finally, we link canopy scattering of SIF to TOC reflectance (Eq. 3.12).

\subsection{Flux interaction with vegetation canopy}

Photons entering the canopy from the top will either go through the canopy via gaps or interact with leaves (or needles). The portion of photons from the incident beam that will not interact with leaves is known as the zero order transmittance $\left(t_{0}\right)$. The complementary portion, the canopy interceptance $\left(i_{0}\right)$, is the portion of photons that will interact with leaves (Smolander and Stenberg, 2005; Huang et al., 2007) (Fig. 3.1). The sum of the zero order transmittance and canopy interceptance is unity. The interception is the first order interactions between incident light and the canopy.

In the first order interactions, photons can either be scattered or absorbed by a leaf, depending on leaf albedo $(\omega)$, which is the sum of leaf reflectance $(\rho)$ and transmittance $(\tau)$. Photons in the photosynthetically 


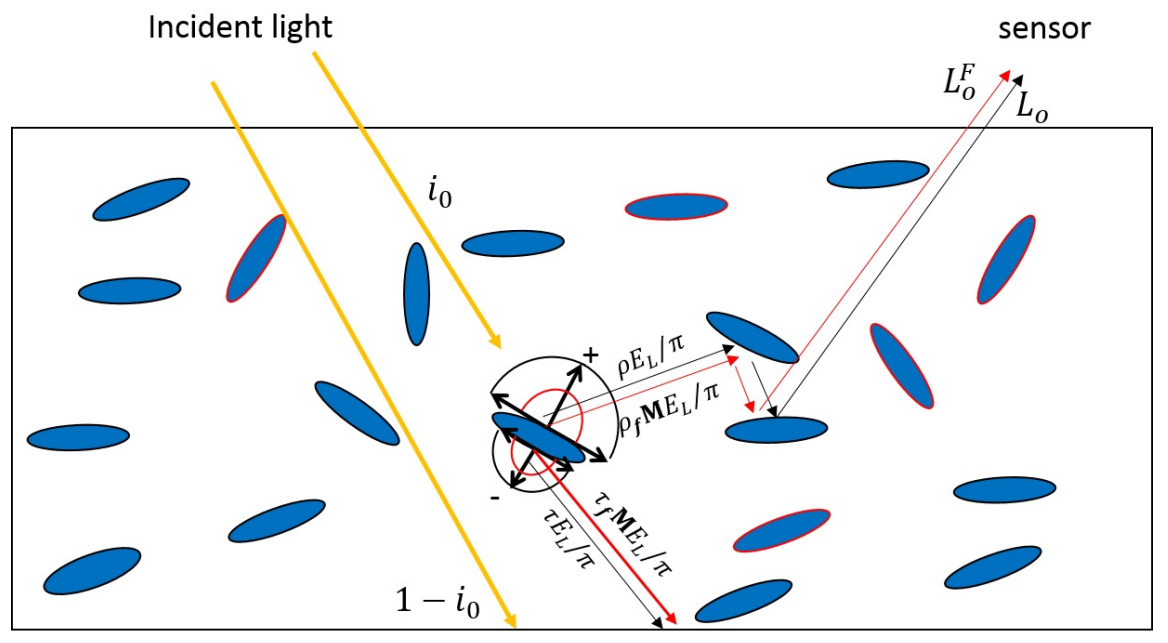

Figure 3.1: The interaction between incident light and canopy. The ellipses represent leaves in the canopy, and red edges indicate the leaves are illuminated directly by the light from the top of the canopy. The fluorescence flux and scattered flux are represented by the red and black curve. The plus and minus signs indicate backward and forward side of a leaf, respectively.

active radiation (PAR) range (i.e., from 400 to $750 \mathrm{~nm}$ ) that hit the leaf, may also excite fluorescence photons in the wavelength range from 640 to $850 \mathrm{~nm}$.

After the first order interactions, photons are scattered and fluorescence photons are emitted from the leaves that are illuminated directly by the (solar or diffuse) incoming light (Fig. 3.1). These photons interact with or escape from the canopy, and part of them will eventually be observed by a sensor at the top of canopy. The probability that photons will be observed, depends on the location of leaf, the direction of flux and wavelength of the photons, but it is independent of the origin of the photons (i.e., an emission or a scattering event). The equality of the radiative transfer of the scattered flux and emitted fluorescence after the first order interactions is the essence of the correlation between TOC reflectance and fluorescence scattering.

Fig. 3.2 summarizes the complete flux interactions with a canopy, starting from the first order interactions (in the middle of the figure). Emission and scattering events occur during the first order interactions (e1 and s1). Part of the scattered and emitted (fluorescence) photons may interact 


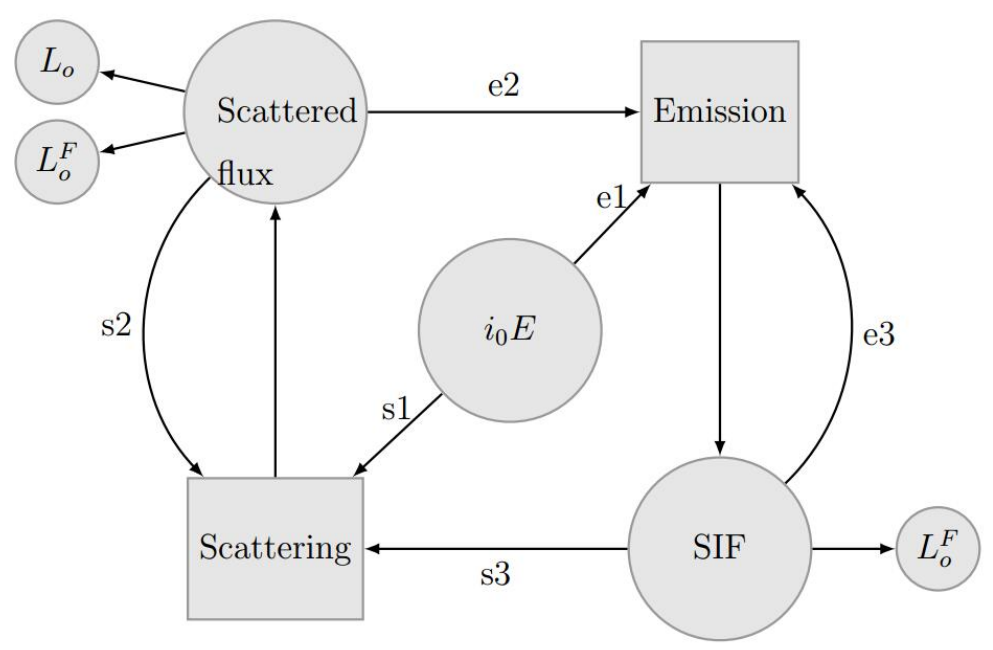

Figure 3.2: Flux and vegetation canopy interaction diagram. The rectangles refer to scattering or fluorescence emission events. The circles refer to fluxes generated from the events. The arrows indicate changes of fluxes. $E$ refers to the incident light. $i_{0}$ is the canopy interceptance, and thus $i_{0} E$ is the light interacting with the canopy. $i_{0} E$ will first be involved in scattering and emission event (s1 and e1), resulting scattered flux and SIF flux, respectively. Part of the fluxes will be observed fluxes by sensor $\left(L_{o}\right.$ and $\left.L_{o}^{F}\right)$. The resulted scattered flux and SIF flux will be involved in scattering and emission events again (s2, s3, e2, e3), and further contribute to the observed fluxes.

with the canopy for multiple times before escaping from the canopy. The multiple interactions can be divided into four classes: (1) scattered (fluorescence) photons are scattered again (s2), (2) emitted fluorescence photons are scattered (s3), (3) scattered photons excite fluorescence (e2), (4) emitted fluorescence photons excite fluorescence (e3).

The total fluorescence emission of the canopy is formed by all the interactions of PAR photons with the leaves (e1, e2 and e3). We found that for obtaining an explicit expression for $\sigma_{F C}=f(R)$, it is both necessary and justified to approximate that the canopy scattering of total emitted SIF $\left(\sigma_{F C}\right)$ can be represented by the canopy scattering of SIF from the first order interactions $\left(\sigma_{F C}^{1}\right)$, which means that excitation by scattered photons $(\mathrm{e} 2)$ and excitation by fluorescence photons (e3) are not considered. The rationale 
for this assumption is twofold. First, most of the fluorescence is generated by sunlit leaves and shaded leaves exposed to the the sky, which are both involved in the first order interactions. Second, the canopy scattering (i.e., a coefficient) of SIF from multiple interactions (e2, e3) $\left(\sigma_{F C}^{m}\right)$ is comparable with, and therefore can be approximated by $\sigma_{F C}^{1}$. Further evidence justifying the approximation is provided in the results section.

\subsection{Calculating fluorescence emission from the first order interactions}

The fluorescence emission at a certain wavelength is excited by photons of different (i.e., shorter) wavelengths interacting with leaves. The relation between excitation of a leaf and emission of fluorescence is described by a so-called excitation-emission matrix, that quantifies the emission spectrum as a function of the excitation spectrum. This matrix depends on the radiative transfer within the leaf, and it can either be measured with dedicated equipments or simulated with leaf RTMs. In the model Fluspect (Vilfan et al., 2016), for example, the relationship between excitation and fluorescence emission is described by a 350 by 211 matrix $\mathbf{M}$ of $1 \mathrm{~nm}$ resolution values, with excitation wavelengths on rows and emission wavelengths on columns. The fluorescence emission from the first order interactions is expressed as

$$
E_{F}\left(\lambda_{f}\right)=i_{0} \int_{400}^{750} \mathbf{M}\left(\lambda_{f}, \lambda_{e}\right) E\left(\lambda_{e}\right) d \lambda_{e}=i_{0} \mathbf{M} E
$$

where $i_{0} E\left(\lambda_{e}\right)$ is the irradiance of PAR photons that are involved in the first order interactions, and $\mathbf{M}\left(\lambda_{f}, \lambda_{e}\right)$ is the excitation-emission matrix. The wavelengths of the excitation and fluorescence emission are $\lambda_{e}$ ranging from 400 to $750 \mathrm{~nm}$ and $\lambda_{f}$ ranging from 640 to $850 \mathrm{~nm}$, respectively. Note that $\mathbf{M} E$ is matrix product that forms a vector. We take the inner product of one row of the matrix $\mathbf{M}$ with of the incident irradiance spectrum $\left(E_{F}\left(\lambda_{f}\right)=i_{0} \mathbf{M} E\right)$ to describe the emission at a certain wavelength.

\subsection{Observed flux and observed SIF flux}

In order to calculate the canopy observed reflected flux and SIF flux, we first provide an expression for the fluxes originating from a single leaf, 
and then integrate the contributions of all the leaves over the canopy.

The incident radiation that hits a leaf $E_{L}(\lambda)$ depends on the location of the leaf $(x, y, z)$, leaf orientation $\left(\varphi_{l}, \theta_{l}\right)$, direction and intensity of the incident light $\left(\varphi_{l}, \theta_{l}\right)$, and is given as

$$
E_{L}(\lambda)=P_{s}(x, y, z) f_{s}\left(\varphi_{l}, \theta_{l}, \varphi_{s}, \theta_{s}\right) E(\lambda)
$$

where $P_{s}$ is a boolean function that indicates whether the leaf is lit by the incident light directly $\left(P_{s}=1\right)$ or not $\left(P_{s}=0\right)$. The projection of a leaf into the direction of the incident light is established by $f_{s}$, which is a function of the azimuth $(\varphi)$ and zenith $(\theta)$ angles of the leaf $(l)$ and of the incident solar light $(s)$ (Verhoef, 1984, 1985).

Assuming that both sides (i.e., the backward side and forward side) of the leaf are Lambertian, the fluxes from each side are isotropic. The photons emanating from the leaf thus consist of transmitted $\tau E_{L}$ and reflected radiation $\rho E_{L}$, backward emission $\rho_{f} \mathbf{M} E_{L}$ and forward emission $\tau_{f} \mathbf{M} E_{L}$, as shown in Fig. 3.1. The quantities $\rho_{f}$ and $\tau_{f}$ describe the relative partitioning of the fluorescence emission over the backward and forward side, and the sum of them is unity in each wavelength (i.e., $\rho_{f}(\lambda)+\tau_{f}(\lambda)=1$ ).

Part of the fluxes scattered from or emitted by the leaves during the first order interactions will be observed by the sensor at top of canopy. For one side of a leaf, the probabilities of the scattered fluxes and of the emitted fluxes from this side that will be observed by the sensor are the same. It is because that the probability that a photon from a leaf at location $(x, y, z)$ will be observed depends on its wavelength and direction rather than its origin (i.e., emission event or scattered event). Therefore, the contribution of a leaf to the observed radiance and to observed fluorescence radiance can be expressed in a similar way.

$$
\begin{gathered}
\Delta L_{o}(\lambda)=\frac{E_{L}}{\pi}\left[\rho f_{o}\left(x, y, z, \lambda, \Omega_{L} \rightarrow \Omega_{o}\right)+\tau f_{o}\left(x, y, z, \lambda,-\Omega_{L} \rightarrow \Omega_{o}\right)\right](3.6) \\
\Delta L_{o}^{F}(\lambda)=\frac{\mathbf{M} E_{L}}{\pi}\left[\rho_{f} f_{o}\left(x, y, z, \lambda, \Omega_{L} \rightarrow \Omega_{o}\right)+\tau_{f} f_{o}\left(x, y, z, \lambda,-\Omega_{L} \rightarrow \Omega_{o}\right)\right]
\end{gathered}
$$


where $f_{o}\left(x, y, z, \lambda, \Omega_{L} \rightarrow \Omega_{o}\right)$ and $f_{o}\left(x, y, z, \lambda,-\Omega_{L} \rightarrow \Omega_{o}\right)$ are the probability of observation of a flux from the leaf's backward and forward side, respectively. From hereon, they are represented by $f_{+}$(backward) and $f_{-}$(forward) for convenience. The wavelength-dependence of the probabilities $f_{-}$and $f_{+}$is due to the involvement of the wavelength-dependent multiple scattering.

The total observed radiance is the sum of the contributions of all the leaves in the canopy (assuming a non-reflecting background). After substituting $E_{L}$ with Eq. 3.5, this sum is:

$$
\begin{aligned}
& L_{o}(\lambda)=\frac{E}{\pi} \sum_{\text {leaves }}\left[P_{s} f_{s} \rho f_{+}+P_{s} f_{s} \tau f_{-}\right] \\
& L_{o}^{F}(\lambda)=\frac{\mathbf{M} E}{\pi} \sum_{\text {leaves }}\left[P_{s} f_{s} \rho_{f} f_{+}+P_{s} f_{s} \tau_{f} f_{-}\right]
\end{aligned}
$$

\subsection{Linking SIF scattering with reflectance}

Using the observed radiances as in Eqs. 3.8 and 3.9, and incident irradiance $E$ and SIF emission ( $i_{0} \mathbf{M} E$, Eq. 3.4), the reflectance and canopy scattering of SIF, as defined earlier in Eqs. 3.1 and 3.2 can be expressed as:

$$
\begin{aligned}
& R(\lambda)=\sum_{\text {leaves }}\left[P_{s} f_{s} \rho f_{+}+P_{s} f_{s} \tau f_{-}\right] \\
& \sigma_{F C}(\lambda)=\frac{1}{i_{0}} \sum_{\text {leaves }}\left[P_{s} f_{s} \rho_{f} f_{+}+P_{s} f_{s} \tau_{f} f_{-}\right]
\end{aligned}
$$

where the variables in front of and behind the plus signs represent contribution of backward and forward side to $L_{o}$ or to $L_{o}^{F}$. It is noticed that both TOC reflectance and canopy scattering of SIF are a combination of $P_{s} f_{s} f_{+}$and $P_{s} f_{s} f_{-}$. This provides the possibility to link them.

Finally, we are interested in the relationship between $R$ and $\sigma_{F C}$. Although reflectance and canopy scattering of SIF are expressed similarly, 
the exact relation is still muddled. There is complexity in the seemingly simple equations 3.10 and 3.11, caused by two facts: (1) A leaf does not scatter photons equally in both directions (forward and backward) and the probability of these photons emanating from these directions to reach the sensor is unequal, (2) $P_{s}, f_{s}, f_{+}$and $f_{-}$are different for each leaf, and different for different canopies. Therefore, the exact quantification of the relationship between $\sigma_{F C}$ and $R$ requires detailed radiative transfer modelling.

However, there are cases in which the relationship between $\sigma_{F C}$ and $R$ can be greatly simplified. First, if $f_{+}=f_{-}$, using that $\omega=\rho+\tau$ and $1=\rho_{f}+\tau_{f}$, we obtain

$$
\sigma_{F C}(\lambda)=\frac{1}{i_{0} \omega} R(\lambda)
$$

Second, if the relative partitioning of the scattered radiation over the two sides of the leaves equals to the relative partitioning of the emitted fluorescence radiation over the two sides of the leaves, such that:

$$
\frac{\rho}{\tau}=\frac{\rho_{f}}{\tau_{f}},
$$

we also obtain the relationship as shown in Eq. 3.12 (i.e., from Eq. 3.13, we know that $\left.\frac{\rho}{\rho_{f}}=\frac{\tau}{\tau_{f}}=\omega\right)$.

In the far-red region (for the far-red SIF), the two conditions mentioned above are both reasonable, such that the relationship between $\sigma_{F C}$ and $R$ in Eq. 3.12 may exist. First, the difference between $f_{+}$and $f_{-}$is smaller in the far-red region than in the red region. Emanating photons in this spectral region are normally involved in multiple interactions. With increasing interaction order, photons tend to 'forget' from which side of the leaf they originate (Mõttus and Stenberg, 2008). Second, Van Wittenberghe et al. (2015) compared the ratio of the backward and forward emission with the ratio of leaf reflectance and transmittance of a number of leaves from 4 species. They found a linear relationship between $\frac{\rho}{\tau}$ and $\frac{\rho_{f}}{\tau_{f}}$ in the far-red region. These two arguments strongly support the validity of the relationship in Eq. 3.12 in the far-red region.

On the contrary, the two conditions mentioned above may not be true in the red region (for the red SIF). First, the contribution of photons from 
the first order interactions in the red region to $L_{o}$ or $L_{o}^{F}$ is mainly from direct observation instead of observation after multiple interactions. As a result, the difference between $f_{+}$and $f_{-}$caused by the different origins (i.e., backward or forward side) is more in the red than in the far-red region. Second, an exponential decay function between $\frac{\tau_{f}}{\rho_{f}}$ and $\frac{1}{\tau}$ was found in the red region (Van Wittenberghe et al., 2015). In fact, the ratios on both sides of Eq. 3.13 are very sensitive to pigment pool of the leaf in the red region. The validity of neither the condition in Eq. 3.13 nor the condition that $f_{+}=f_{-}$ is guaranteed in the red region. Therefore, the relationship between $\sigma_{F C}$ and $R$ of Eq. 3.12 may not hold for red SIF.

\section{SCOPE simulation method}

We tested the relationship between canopy scattering of SIF $\left(\sigma_{F C}\right)$ and TOC reflectance $(R)$ as shown in Eq. 3.12 by using simulations with the SCOPE model (Van der Tol et al., 2009) for a number of scenarios. Several assumptions were also tested by using SCOPE simulations. In total, 1800 scenarios of combinations of different leaf properties, canopy structure or sun zenith angles were generated.

We first studied the spectra of canopy scattering of SIF $\left(\sigma_{F C}\right)$ and tested the sensitivity to changing of LAI and leaf chlorophyll content, which are two key canopy properties. Further, we focused on the results for canopy scattering of SIF at $687 \mathrm{~nm}$ and $760 \mathrm{~nm}\left(F_{687}\right.$ and $\left.F_{760}\right)$, representing the red and far-red fluorescence used in remote sensing of vegetation (Meroni et al., 2009).

\subsection{SCOPE model}

SCOPE is a model for homogeneous vegetation and consists of a leaf RTM, several canopy RTMs and an energy balance model. At the leaf level, Fluspect (Vilfan et al., 2016), which is based on PROSPECT (Jacquemoud and Baret, 1990), simulates leaf reflectance $(\rho)$, transmittance $(\tau)$, and fluorescence emission of the both forward and backward side. At the canopy level, RTMo and RTMf, which are two SAIL (Verhoef, 1984) based models, 
compute the radiative transfer of incident radiation and emitted fluorescence, respectively.

SCOPE provides the necessary output to test the relationship we found. TOC reflectance $(R)$ and fluorescence $\left(L_{o}^{F}\right)$ are two direct outputs of SCOPE. Canopy fluorescence emission $\left(E_{F}\right)$ is computed by SCOPE, but it is not stored as output. We modified SCOPE version 1.70 to store it as output. We computed the canopy scattering of SIF for each scenario according to Eq. 3.2. Canopy interceptance $\left(i_{0}\right)$ is a spectral invariant determined by canopy LAI and leaf inclination distribution (Smolander and Stenberg, 2005). The literature about the spectral invariant theory provides equations for $i_{0}$, (e.g., Smolander and Stenberg, 2005; Huang et al., 2007). In SCOPE, it is calculated as $1-\exp (-k L)$, where $k$ is the extinction coefficient and $L$ is canopy leaf area index. The extinction coefficient is determined by sun zenith angle and leaf inclination distribution. We used the canopy interception as simulated by SCOPE, and modified the code to save it as output. For leaf albedo, we used the sum of leaf reflectance and transmittance as simulated by Fluspect.

\subsection{Database generation}

The input of SCOPE for the 1800 scenarios comprises of 60 combinations of leaf properties, 10 combinations of canopy structure parameters, and 3 sun zenith angles (Table 3.1). The values of parameters for leaf properties, canopy structure and sun position were chosen within the recommended ranges in SCOPE (Van der Tol et al., 2009; Yang et al., 2017) with non-linear steps. The viewing zenith angle was $0^{\circ}$ (i.e. at nadir), and a non-reflecting background and a typical incident irradiance spectrun were used (i.e., default setting in the SCOPE model, see supplementary materials).

TOC reflectance, TOC SIF, and total SIF emission of the 1800 scenarios were simulated with SCOPE. Leaf reflectance $(\rho)$, transmittance $(\tau)$, fluorescence emission on the backward and forward side, and thus $\rho_{f}$ and $\tau_{f}$ were simulated for the 60 leaves. 
Table 3.1: Summary of SCOPE inputs applied for the generation of the database

\begin{tabular}{llll}
\hline Parameter & Explanation & Unit & Values \\
\hline$C_{a b}$ & Chlorophyll $a+b$ content & $\mu \mathrm{g} \mathrm{cm}^{-2}$ & $5,10,20,40,80$ \\
$C_{d m}$ & Leaf mass per unit area & $\mathrm{g} \mathrm{cm}^{-2}$ & $0.01,0.02$ \\
$C_{w}$ & Equivalent water thickness & $\mathrm{cm}$ & $0.015,0.03$ \\
$N$ & Leaf structure parameter & - & $1,1.5,2$ \\
LAI & Leaf area index & - & $0.5,1,2,3,6$ \\
LIDFa & Leaf inclination function parameter a & - & $-0.5,0.5$ \\
$\theta_{s}$ & sun zenith angle & $\circ$ & $30,45,60$ \\
\hline
\end{tabular}

\subsection{Testing the assumptions}

The assumption that the canopy scattering of SIF $\left(\sigma_{F C}\right)$ can be approximated by scattering of SIF from the first order interactions $\left(\sigma_{F C}^{1}\right)$ was tested by using SCOPE simulations. We also compared $\frac{\rho}{\tau}$ and $\frac{\rho_{f}}{\tau_{f}}$ from Fluspect simulations.

To test the two statements in section 2.2 that 1 ) the observed SIF from canopy scattering is most from the first order interactions, and 2) scattering of SIF from first order interactions $\left(\sigma_{F C}^{1}\right)$ and multiple interaction $\left(\sigma_{F C}^{m}\right)$ are comparable, two sets of simulations were conducted. In these simulations, SIF emission from the first order interactions $\left(E_{F}^{1}\right)$ and from the multiple interactions $\left(E_{F}^{m}\right)$ of the 1800 scenarios were separately simulated as well as the correspondingly observed $\operatorname{SIF}\left(L_{o}^{F 1}\right.$ and $\left.L_{o}^{F m}\right)$ and scattering coefficients $\left(\sigma_{F C}^{1}\right.$ and $\left.\sigma_{F C}^{m}\right)$. This was done by modifying the input to RTMf in the code of SCOPE, notably by removing the scattered light and the direct incident light distributed in the canopies in RTMf, respectively. The light distribution inside the canopy was simulated in RTMo and later used as input of RTMf for fluorescence simulations. SIF emission during the first order interactions was excited by the direct solar flux (i.e., we used the direct solar beam as incident light). Therefore, by removing the scattered light in the input of RTMf, we obtained SIF emission from the first order interactions, and the corresponding SIF observed at top of canopy and thus computed $\sigma_{F C}^{1}$. Conversely, SIF emission from the multiple interaction was excited by the scattered light. By removing direct light distributed in the canopy in RTMf, we obtained $E_{F}^{m}, L_{o}^{F m}$ and $\sigma_{F C}^{m}$. We compared the canopy SIF emission from the first 
order interactions and from the multiple interactions of the scenarios as well as TOC SIF observed as simulated with SCOPE. Further, $\sigma_{F C}^{1}$ and $\sigma_{F C}^{m}$ were compared. The simulated results of the 1800 scenarios were grouped by canopy LAI. There were 360 simulations for each unique LAI value. The mean and standard derivation of each group were computed and compared. It should be noted that SCOPE simulates fluorescence excited by scattered photons (e2 in Fig. 3.2), but it neglects the much smaller flux of fluorescence excited by fluorescence photons (e3).

To test the condition that $\frac{\rho}{\tau}=\frac{\rho_{f}}{\tau_{f}}$ in section 2.4, we compared the two ratios simulated from Fluspect for the 60 leaves comprising all the combinations of the leaf parameters in Table. 2.2. Leaf reflectance and transmittance were direct outputs of Fluspect, and $\frac{\rho_{f}}{\tau_{f}}$ were computed as the ratio between backward and forward fluorescence emission of the leaves.

\subsection{Validation of the relationship between reflectance and canopy scattering of SIF}

To test whether $\frac{\rho}{\tau}=\frac{\rho_{f}}{\tau_{f}}$ (Eq. 3.13) is a sufficient condition of the relationship between $\sigma_{F C}$ and $R$ in Eq. 3.12, two types of simulations were carried out, each consisting of the 1800 scenarios described above (3600 simulations in total). In the first group of 1800 simulations (group1), we modified the Fluspect output of the 60 leaves in such a way that the assumption in Eq. 3.13 was fulfilled. The leaf albedo of the 60 leaves was calculated as the sum of reflectance and transmittance, and likewise, fluorescence emission was calculated as the sum of the forward and the backward emission. Leaf reflectance and transmittance were then recalculated as $\omega / 2$ each, and likewise, forward and backward fluorescence as half of the total fluorescence each (i.e., $\rho=\tau=\omega_{L} / 2$ and $\rho_{f}=\tau_{f}=0.5$ ). Note that taking equal $\rho$ and $\tau$, and $\rho_{f}$ and $\tau_{f}$ is a special case among all cases that comply with Eq. 3.13. These leaves are considered as synthetic leaves. In the second group of simulations (group-2), the manipulations above was not carried out, and leaf reflectance and transmittance, and forward and backward emission as simulated by Fluspect were used directly. These leaves 
are considered as Fluspect leaves.

We validated the relationship of canopy SIF scattering and reflectance in Eq. 3.12 for both red and far-red SIF. Both the group-1 and group-2 simulations from SCOPE were used to evaluate the relationship.

\section{Simulation results}

\subsection{Canopy scattering of SIF}

The canopy scattering of SIF $\left(\sigma_{F C}\right)$ was spectrally dependent (Fig. 3.3). The value of $\sigma_{F C}$ was much higher in the near-infrared region than in the red region. Furthermore, it increased with LAI in the near-infrared region, but decreased in the red region.

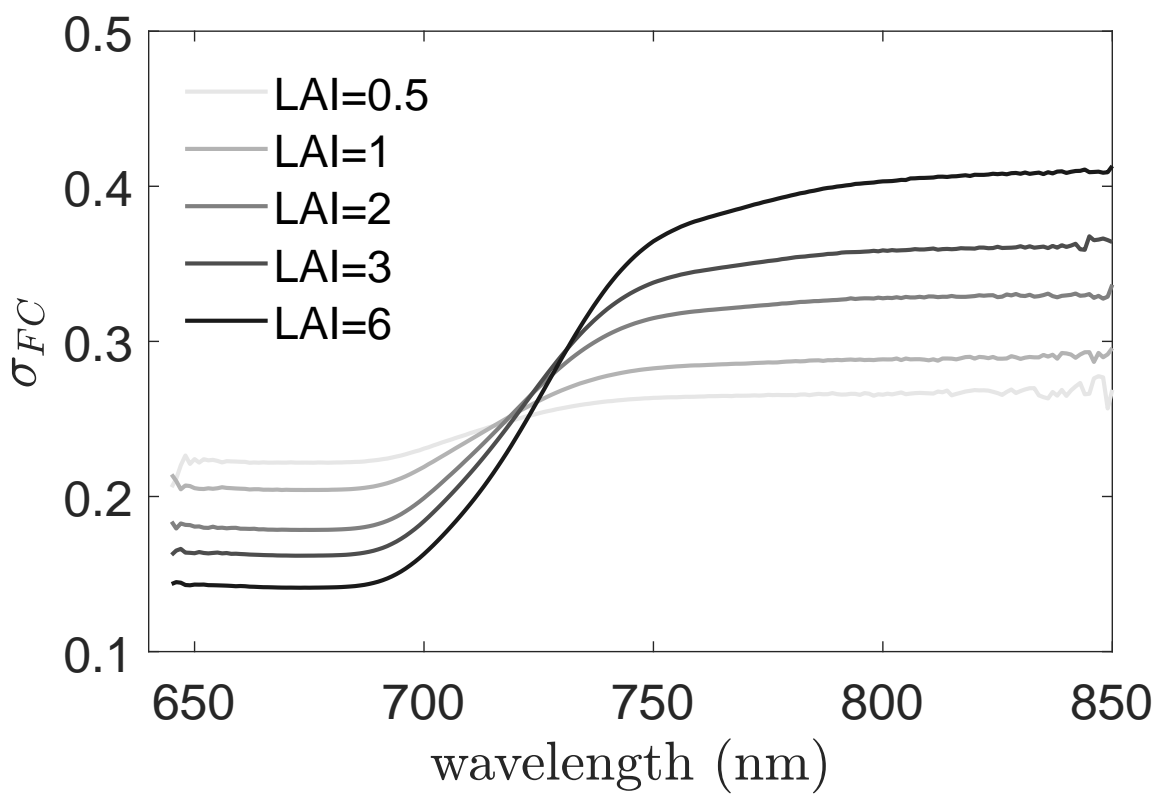

Figure 3.3: The canopy scattering of SIF $\left(\sigma_{F C}\right)$ in the spectral region from $640 \mathrm{~nm}$ to $850 \mathrm{~nm}$ simulated by using SCOPE. In the simulations, LAI was set to $0.5,1,2,3$ or 6 . Leaf chlorophyll $C_{a b}=40 \mu \mathrm{g} \mathrm{cm}^{-2}$. Leaf structure parameter $N=1.5$. Leaf mass $C_{d m}=0.01 \mathrm{~g} \mathrm{~cm}^{-2}$. Equivalent water thickness $C_{w}=0.015 \mathrm{~cm}$. LIDFa $=-0.5$. Sun zenith angle $\theta_{s}=30^{\circ}$.

Leaf chlorophyll content strongly affected $\sigma_{F C}$ in the red region, but had almost no impact on $\sigma_{F C}$ in the near-infrared region (Fig. 3.4). The higher leaf chlorophyll content resulted in lower $\sigma_{F C}$ in the red region. 


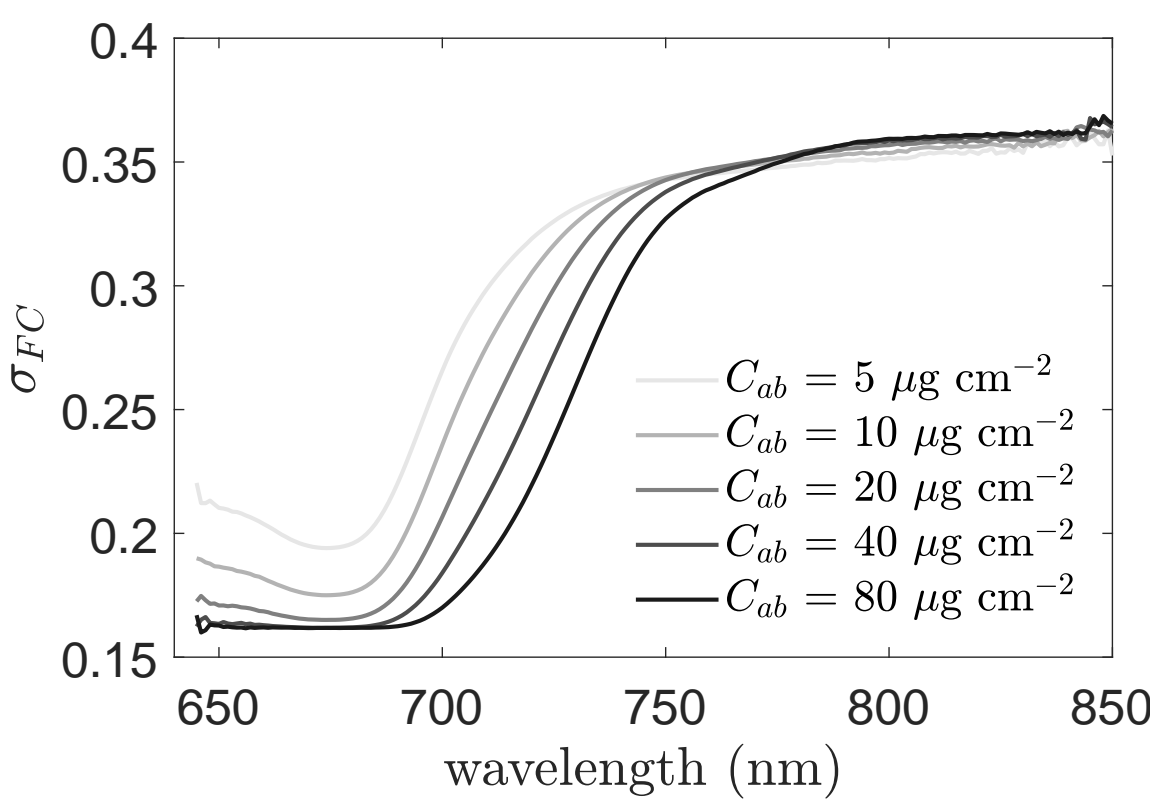

Figure 3.4: The canopy scattering of SIF $\left(\sigma_{F C}\right)$ in the spectral region from $640 \mathrm{~nm}$ to $850 \mathrm{~nm}$ simulated by using SCOPE. In the simulations, Leaf chlorophyll $C_{a b}$ was set to $5,10,20,40$ or $80 \mu \mathrm{g} \mathrm{cm}^{-2}$. LAI $=3$. Leaf structure parameter $N=1.5$. Leaf mass $C_{d m}=0.01 \mathrm{~g} \mathrm{~cm}^{-2}$. Equivalent water thickness $C_{w}=0.015 \mathrm{~cm}$. LIDFa $=-0.5$. Sun zenith angle $\theta_{s}=30^{\circ}$.

\subsection{SIF from first order and multiple interactions}

Fig. 3.5 shows that the SIF emission from the first order interactions between the incident light and canopies was much higher than SIF emission from the multiple interactions (i.e., emitted by scattered PAR photons) at both $687 \mathrm{~nm}$ and $760 \mathrm{~nm}$. SIF observed at the top of the canopies was also mostly from the first order interactions at both $687 \mathrm{~nm}$ and $760 \mathrm{~nm}$ (Fig. 3.6). Both canopy emitted SIF and observed SIF increased with the increasing LAI at both $687 \mathrm{~nm}$ and $760 \mathrm{~nm}$. 

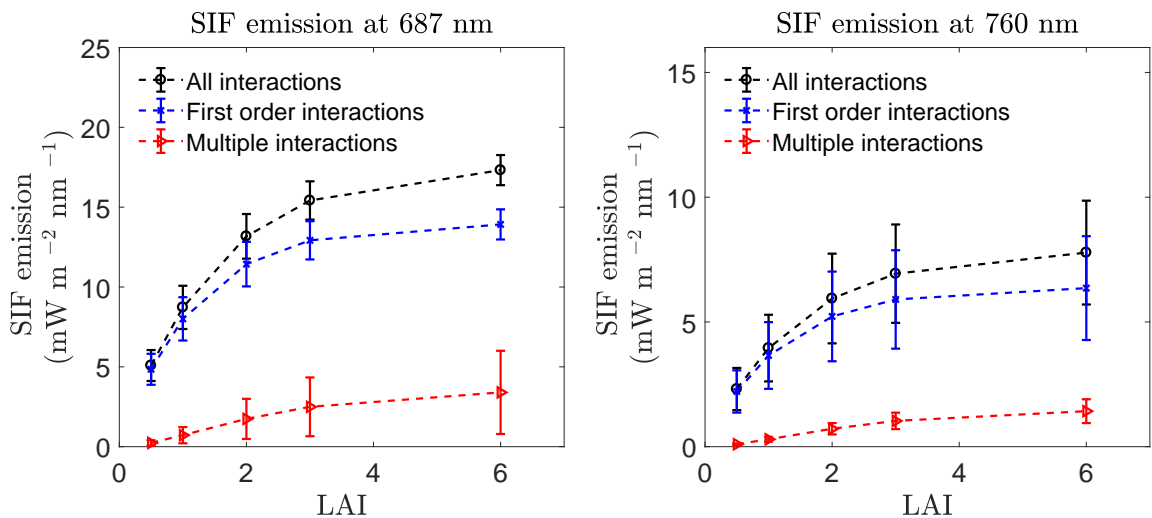

Figure 3.5: SIF emission at $687 \mathrm{~nm}$ and $760 \mathrm{~nm}$ from all the interactions, the first order interactions and multiple interactions changing with LAI. The error bars represent the total range of variation of the 360 scenarios with the same LAI but different leaf properties, leaf orientations or sun zenith angles. Note: SIF from the first order interactions refers to the fluorescence emission by incident light directly. SIF from the multiple interactions is the fluorescence emission excited by scattered light.
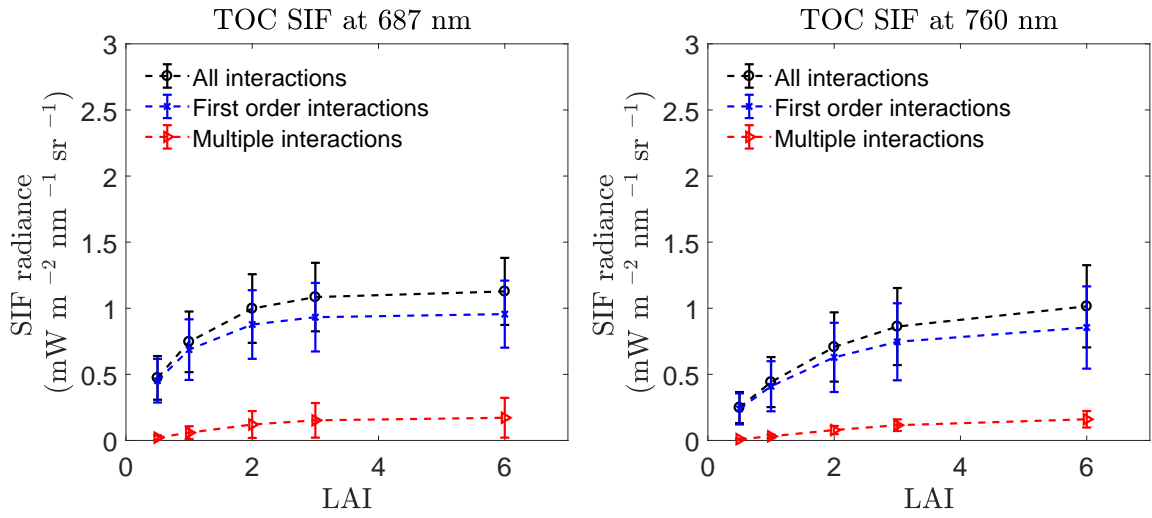

Figure 3.6: Radiance of top-of-canopy (TOC) SIF observed at $687 \mathrm{~nm}$ and $760 \mathrm{~nm}$ from all the interactions, the first order interactions and multiple interactions changing with LAI. 
The scattering of whole canopy SIF $\left(\sigma_{F C}\right)$ closely matched with the scattering of SIF from the first order interactions alone $\left(\sigma_{F C}^{1}\right)$ (Fig. 3.7). The difference between $\sigma_{F C}^{1}$ and $\sigma_{F C}^{m}$ was less than 30\%. Canopy scattering of far-red SIF increased but that of red SIF decreased with LAI as also shown in Fig. 3.3.
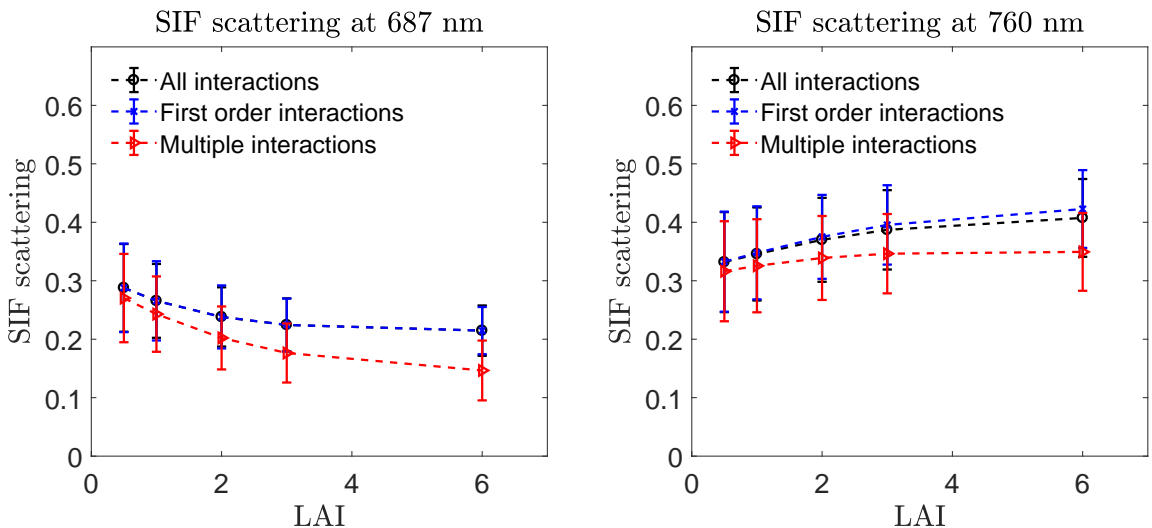

Figure 3.7: Canopy scattering of SIF at $687 \mathrm{~nm}$ and $760 \mathrm{~nm}$ from all the interactions, the first order interactions and multiple interactions changing with LAI.

\subsection{Radiation distribution over two sides of leaves}

The distributions of scattered and emitted photons over the two sides of the leaf as simulated with Fluspect were similar in the far-red spectral region, but this symmetry was much less for red region (Fig. 3.8). Both $\rho / \tau$ and $\rho_{f} / \tau_{f}$ were sensitive to leaf structure $(N)$ at $760 \mathrm{~nm}$. The pigments content had no impact on $\rho / \tau$ at $760 \mathrm{~nm}$. On the contrary, the two quantifies at $687 \mathrm{~nm}$ were sensitive to both leaf structure and pigments content.

\subsection{Relationship between TOC reflectance and canopy scattering of SIF}

Fig. 3.9 shows that $\sigma_{F C}$ predicted from canopy reflectance (i.e., $\sigma_{F C}$ $\left.=\frac{R}{i_{0} \omega}\right)$ from the group-1 simulations was in almost perfect agreement with $\sigma_{F C}$ simulated with SCOPE at both $687 \mathrm{~nm}$ and $760 \mathrm{~nm}$, with both $R^{2}$ 

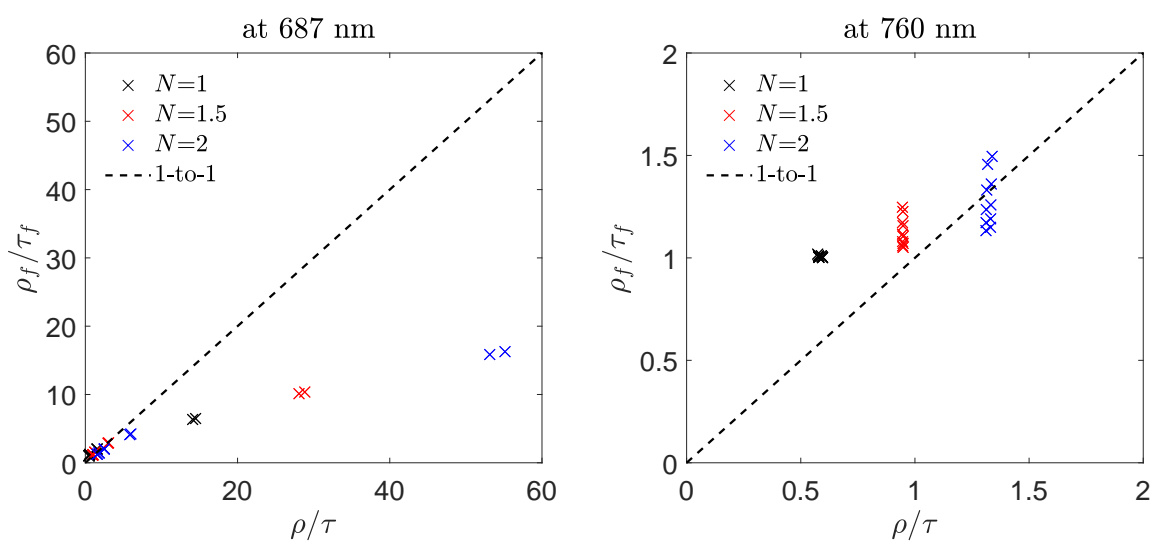

Figure 3.8: The comparison of partitioning of scattered radiation $(\rho / \tau)$ and partitioning of emitted SIF $\left(\rho_{f} / \tau_{f}\right)$ over the two sides of leaves at $687 \mathrm{~nm}$ and $760 \mathrm{~nm}$ simulated with Fluspect. Simulations with the same leaf structure parameter $(N)$ are marked with the same colour.

$=0.98$. Small excursions from 1-1 line were found in only few of the 1800 scenarios.

Fig. 3.10 shows that the correlation between $\sigma_{F C}$ and $\frac{R}{i_{0} \omega}$ was stronger for $760 \mathrm{~nm}$ than for $687 \mathrm{~nm}$ when the Fluspect leaves were used (group2 scenarios). For the far-red $\operatorname{SIF}\left(F_{760}\right), \sigma_{F C}$ and $\frac{R}{i_{0} \omega}$ showed significant positive correlation with $R^{2}=0.772$. This was obviously lower than for group-1 simulations, but still significant. The correlation was poor for red SIF $\left(F_{687}\right)$. Although $\sigma_{F C}$ and $\frac{R}{i_{0} \omega}$ were still positively correlated, the excursions from the 1:1 line were substantial in many scenarios. However, for a individual leaf, $\sigma_{F C}$ and $\frac{R}{i_{0} \omega}$ were also linear when canopy structure (LAI and LIDFa), and sun zenith angle were changing. The slope of the relationship between $\sigma_{F C}$ and $\frac{R}{i_{0} \omega}$ was leaf dependent. In Fig. 3.10, we only randomly marked three individual leaves for readability of the figure (for the correlation of individual leaves, see the supplementary material). 

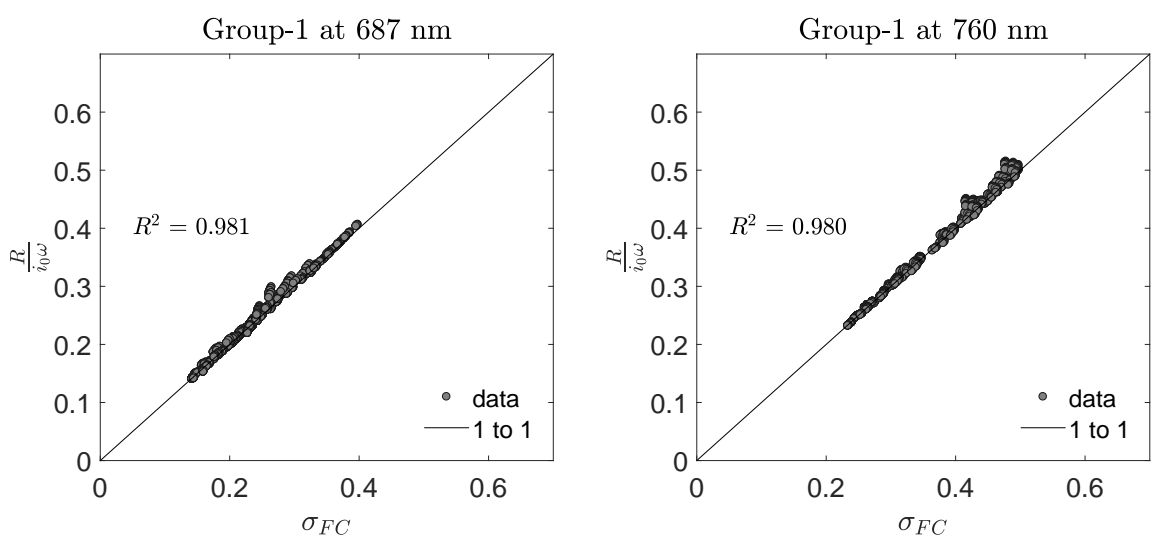

Figure 3.9: The correlation between canopy scattering of SIF $\left(\sigma_{F C}\right)$ and $\frac{R}{i_{0} \omega}$ at $687 \mathrm{~nm}$ and $760 \mathrm{~nm}$ for the group-1 (synthetic leaves) scenarios. Each point represents one scenario. Every leaf in the group had equal reflectance and transmittance $(\rho=\tau=\omega / 2)$, and equal backward and forward fluorescence emission. Note: $R$ is canopy reflectance, $i_{0}$ is the canopy interceptance and $\omega$ is the leaf albedo.
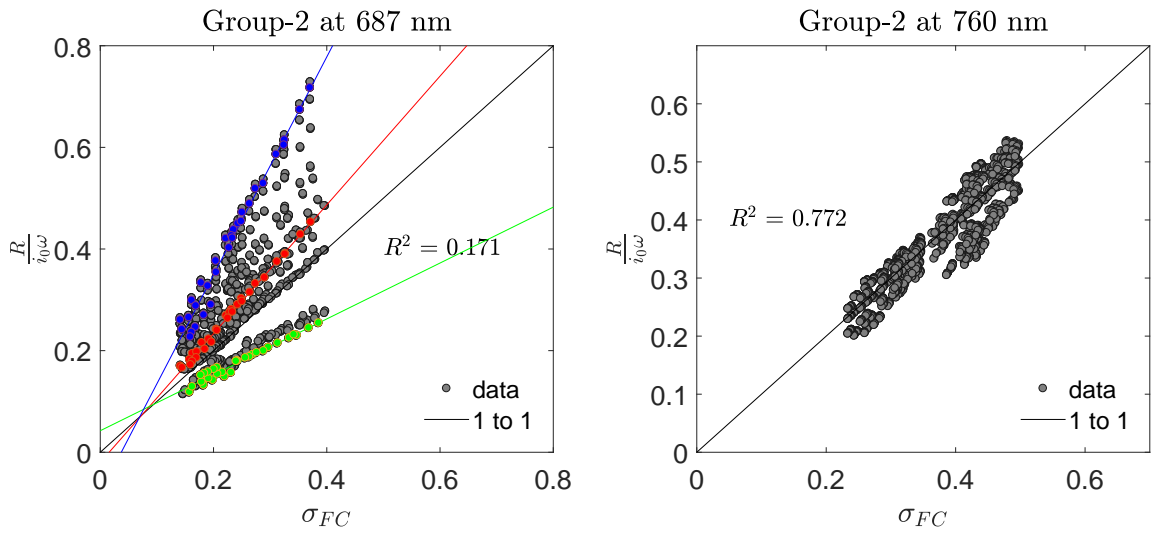

Figure 3.10: The correlation between canopy scattering of SIF $\left(\sigma_{F C}\right)$ and $\frac{R}{i_{0} \omega}$ at $687 \mathrm{~nm}$ and $760 \mathrm{~nm}$ for the group-2 (Fluspect leaves) scenarios Each point represents one scenario. Simulations of scenarios that have the same leaf are highlighted with the same colour. Three individual leaves are respectively marked as red, blue and green for illustration, while the remaining 57 leaves are marked as gray. Note: $R$ is canopy reflectance, $i_{0}$ is the canopy interceptance and $\omega$ is the leaf albedo. 


\section{Discussion}

\subsection{Interpretation by using spectral invariant theory}

The analogy of radiative transfer of intercepted incident light and emitted SIF allowed us to derive the relationship between reflectance $(R)$ and canopy scattering of SIF $\left(\sigma_{F C}\right)$, merely by reasoning rather than detailed modelling. The relation in Eq. 3.12 can also be obtained from existing spectral invariant recollision theory, as follows.

Smolander and Stenberg (2005) introduced the recollision probability $p$ to calculate total scattering $s(\lambda)$ of a canopy (bounded underneath by a non-reflecting surface):

$$
s(\lambda)=i_{0} \frac{(1-p) \omega(\lambda)}{1-p \omega(\lambda)}
$$

where the recollision probability $p$ is defined as the probability that a photon, after having survived an interaction with a canopy element, will interact with the canopy again.

A second spectral invariant, the directional escape probability $\rho(\Omega)$, quantifies the portion of scattered photons that escapes via gaps in the direction of viewing (Huang et al., 2007). Canopy directional reflectance can be expressed as (Schull et al., 2007; Köhler et al., 2018):

$$
R=i_{0} \rho(\Omega) \frac{\omega(\lambda)}{1-p \omega(\lambda)}
$$

Canopy scattering of SIF can be expressed by using the spectral invariants as well. A TOC SIF observation includes contributions from a series of progressively smaller components: 1) emitted SIF photons that directly escape and are observed by the sensor $(\rho(\Omega)) ; 2)$ emitted SIF photons that interact once with the canopy before escaping and being observed $(p \omega(\lambda) \rho(\Omega)) ; 3)$ emitted SIF photons that interact twice before escaping and being observed $\left(p^{2} \omega(\lambda)^{2} \rho(\Omega)\right)$, etc. The total canopy scattering of SIF is the 
sum of the contributions, which is given as a geometric series:

$$
\sigma_{F C}(\lambda)=\rho(\Omega)+p \omega(\lambda) \rho(\Omega)+p^{2} \omega(\lambda)^{2} \rho(\Omega)+\ldots=\frac{\rho(\Omega)}{1-p \omega(\lambda)}
$$

Note that both $\rho(\Omega)$ and $p$ are spectral invariant and depend on canopy structure. Dividing Eq. 3.16 by Eq. 3.15 eliminates $\rho(\Omega)$ and $p$, and results in Eq. 3.12.

Eq. 3.12 can thus be obtained from spectral invariant theory as well, but the theoretic derivation in section 2 is more general. In the spectral invariant theory, leaf single scattering is quantified by leaf albedo $(\rho+\tau)$, while $\rho$ and $\tau$ are not considered separately. However, the difference $\rho-\tau$ appears to be essential: The asymmetry of leaf scattering explains why the simple relationship between TOC reflectance and scattering of SIF is valid for the far-red region, but not for red region.

The canopy scattering of SIF is also linked with the directional area scattering factor (DASF) (Knyazikhin et al., 2013), which is a combination of the three spectrally invariant parameters (i.e., $i_{0}, p$ and $\rho$ ).

$$
\operatorname{DASF}=\frac{\rho(\Omega) i_{0}}{1-p}
$$

DASF is the TOC directional reflectance if the foliage does not absorb radiation (i.e., $\omega=1$ ). At weakly absorbing wavelengths, for example, in near-infrared region, $\sigma_{F C}$ can be approximated by $\mathrm{DASF} / i_{0}$ or by $R / i_{0}$ (i.e., $\operatorname{DASF}=R$, when $\omega=1)$.

\subsection{Canopy scattering of SIF and reflectance}

Canopy scattering of SIF and reflectance are affected by leaf properties and canopy structure in a comparable way. The pigments content in a leaf mostly affects $\sigma_{F C}$ in the red region, but not in the near-infrared region. For example, $\sigma_{F C}$ in the near-infrared region does not change with leaf chlorophyll content (Fig. 3.4). Likewise, near-infrared reflectance is not sensitive to leaf chlorophyll content (Jacquemoud et al., 2009). Both $\sigma_{F C}$ (Fig. 3.2) and $R$ (Jacquemoud et al., 2009) respond to canopy LAI differently in the red and near-infrared region: they increased with LAI in the near-infrared region, 
but decreased in the red region. For the red SIF, the signal at top of canopy is mainly from the emitted SIF from leaves that are directly observed via gaps. The contribution from emitted SIF after interacting with leaves again is rather small. Higher LAI normally associates with lower canopy gap fraction. Therefore, a smaller portion of the emitted SIF will be observed, and thus a smaller $\sigma_{F C}$. On the contrary, far-red SIF observed strongly affected by multiple scattering, which increases with LAI. The sensitivity of $\sigma_{F C}$ and $R$ to canopy structure and leaf properties is, however, not exactly the same. For example, the red reflectance is less sensitive to LAI than the scattering of red SIF comparing Fig. 3.3 to Fig. 3 in Jacquemoud et al. (2009) or to Fig. $2 \mathrm{~d}$ in Jacquemoud (1993). It is because that $\sigma_{F C}$ in this region is mostly determined by the canopy gap fraction, while TOC reflectance is determined jointly by the light interception $\left(i_{0}\right)$ and gap fraction. Although lower canopy fraction results in a better chance of the radiation from leaves been observed, it also results in a smaller $i_{0}$. The smaller $i_{0}$ and higher gap fraction have an opposite effect on the TOC red reflectance, which counteracts the impact of LAI on red reflectance.

\subsection{Validity of the relationship between canopy scattering of SIF and reflectance}

The relationship between $\sigma_{F C}$ and TOC reflectance relies on the analogy of radiative transfer of intercepted incident light and emitted SIF. It has a physical basis. In our derivation, it is important to separate the first order interactions and multiple interactions. The first order interactions are the interception of incident light, which results in the scattered radiation and fluorescence emission. Further scattering by subsequent interactions determines the probability that the photons resulting from the first order interactions can be observed at the top of canopy from a specific direction. This further scattering is indifferent to the source of the photons (i.e. scattering or emission), and this is the basis for the relationship between SIF scattering and reflectance, namely that the scattering of SIF is proportional to the ratio of reflectance to the product of the canopy interceptance and 
the leaf albedo $\left(R=i_{0} \omega \sigma_{F C}\right)$.

Several assumptions underlay our derivation. In deriving the equations for reflectance and SIF scattering (Eqs. 3.10 and 3.11), we neglected two sources of fluorescence produced in higher order interactions, notably the excitation of fluorescence by scattered light and the excitation of fluorescence by fluorescence photons (e2 and e3 in Fig. 3.2). This simplification appears to be justified: the scattering of SIF as simulated by SCOPE matches closely with the scattering of SIF from the first order interactions $\left(\sigma_{F C}^{1}\right)$ (Fig. 3.7). Although SCOPE also neglects the (very small) flux of excitation of fluorescence by fluorescence photons (e3), it does account for the excitation of fluorescence by scattered light (e2). In the development and validation of the relationship, we did not consider the soil background effects. There are scattering effects from soil (indirect contribution) and direct contribution to observed signals. The soil background has the identical scattering effects on observed flux and on SIF as included in $f_{+}$and $f_{-}$. This makes no change to the relationship we found. However, the sunlit soil directly contributes to observed flux, but not directly contribute to observed SIF (i.e., it does not emit SIF photons). The direct contribution of sunlit soil to TOC reflectance (Eq. 3.10) can be expressed as $\left(1-i_{0}\right) r_{s} P_{o}$, where $\left(1-i_{0}\right)$ is the probability of photons will go through the canopy directly (zero order transmittance, $t_{0}$ ), $r_{s}$ is soil reflectance and $P_{o}$ is the probability that the soil will be observed. This contribution may affect the relationship and change with canopy coverage, but for the exact impact we need further investigations.

More critical is the directionality (i.e., forward or backward) of the fluxes generated by leaves in the first order interactions. If the distribution of scattered light over reflection and transmission is different from the distribution of emitted fluorescence over the two sides of the leaf, then our simplified relation of Eq 3.12 does not hold, because forward and backward propagated light scatter differently in subsequent interactions. In that case, no simple mathematical expression can describe the relationship between fluorescence scattering and reflectance. The asymmetry between the distribution of scattered and emitted photons over the two sides of the leaf, combined 
with the fact that the canopy scattering $f_{-}$and $f_{+}$are unequal in the red region, make the relationship between canopy scattering of fluorescence and reflectance deviate from the general form of Eq. 3.12. This relation is leaf property dependent in the red region and may depend on the arrangement of pigments in the leaf (Vogelmann and Han, 2000).

Interestingly, $\frac{R}{i_{0} \omega}$ and $\sigma_{F C}$ are still proportional in the red (but not equal) for individual leaves in the 30 simulated canopies, but the slope of the relationship varies with leaf structure and pigment composition (see supplementary information). A more general expression for the scattering of fluorescence in the red region would require the inclusion of scattering within the leaf in the analysis, which would be an extremely useful line to investigate further.

In the derivation of Eq. 3.12, we did not parameterize the structure of the canopy (such as leaf shape and leaf inclination distribution, fractional cover), and thus, did not make assumptions on the form and representation of the canopy in a model. This makes our results generic, and Eq. 3.12 should be applicable to a wide range of canopies. For the validation of the equation, we used a turbid canopy model (SCOPE) in which $P_{s}, f_{s}, f_{+}$and $f_{-}$are functions of the vertical position of the leaf in the canopy (in units of LAI), but are independent to the horizontal position $(x, y)$, but the applicability of Eq. 3.12 is not limited to canopies that can be considered as a turbid medium. The fact that the same relationship can be derived using spectral invariant theory supports the potential application in needle forest (Rautiainen and Stenberg, 2005) and clumped canopies (Stenberg and Manninen, 2015). We even expect that the relationship is more promising for needle forest, since the asymmetry in needles is less obvious than in leaves. However, the general relationship of Eq. 3.12 relies on the assumption that leaves have equal properties in the whole canopy. Although this is a common assumption in many radiative transfer models, it could be relaxed in some detailed radiative transfer models for further testing of the relationship between SIF scattering and reflectance. 


\subsection{Validation of the relationship}

Direct validation of the relationship in Eq. 3.12 requires the measurements of TOC reflectance, canopy interceptance, leaf albedo and canopy scattering of SIF. The last one $\left(\sigma_{F C}\right)$ can be computed knowing the total emitted SIF of the canopy and TOC SIF observed. Canopy SIF measurements are retrieved from various platforms by using the FLD method. It is also possible to measure total emission by using some dedicated instruments, such as FluoWAT (Van Wittenberghe et al., 2012; Cendrero-Mateo et al., 2015), but a representative number of leaves needs to be measured. Besides of the labor consuming, the requirement of simultaneous measurements of leaf SIF and canopy SIF makes the direct validation difficult.

Indirect validation by looking into the directional effects on SIF and reflectance is more practical. According to the relationship we found, the viewing angle effects on TOC SIF and reflectance are analogous. SIF observed from different angles differs from each other due to the different canopy scattering of the emitted SIF. The variation in view angles does not affect the total emission, but only the canopy scattering of SIF which is linked with reflectance (Eq. 3.12). Field measurements presented in Liu et al. (2016) provides evidence for the similar viewing angle effects on SIF and on reflectance. A demonstrative simulation with SCOPE confirms the identical viewing zenith angle effects on SIF and on reflectance, at both $687 \mathrm{~nm}$ and $760 \mathrm{~nm}$ (Fig. 3.11). A recent study by Köhler et al. (2018) also reported a similar but not identical directional effects on GOME-2 far-red SIF and near-infrared reflectance. The difference was caused by the variation of solar angle. In Köhler et al. (2018) study, both solar angle and viewing angle varied (i.e., their changes were quantified by the phase angle). In contrast to viewing angle, the effect of solar zenith angle is not identical for reflectance and SIF. The solar zenith angle affects the incident light intensity and the canopy interception of the incident light $\left(i_{0}\right)$, and thus SIF emission. Therefore, the sun zenith angle effects the slope of SIF versus reflectance via incident light condition, according to Eq. 3.12. 

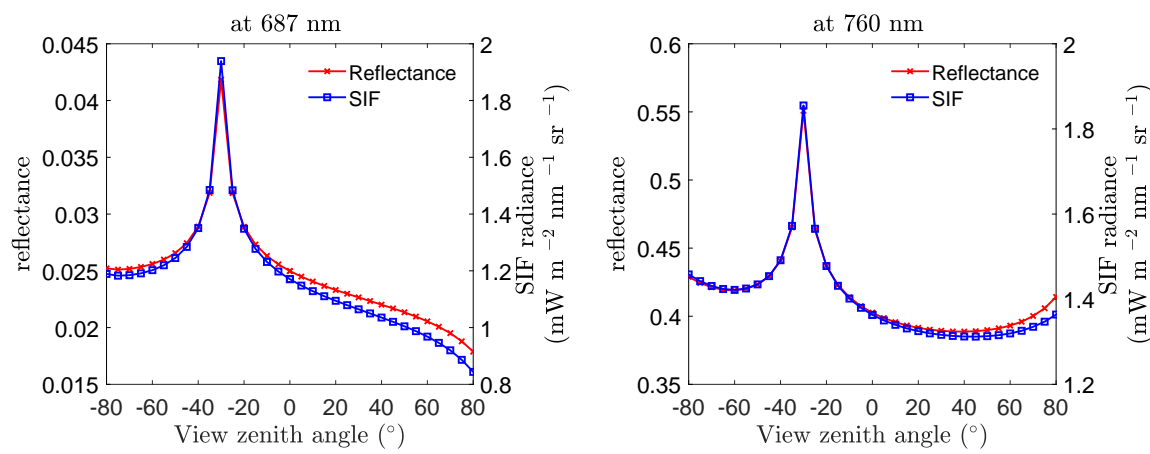

Figure 3.11: View zenith angle (VZA) effects on reflectance and SIF at 687 $\mathrm{nm}$ and $760 \mathrm{~nm}$ simulated with SCOPE. Negative values of the VZA represent the backward direction, and positive values represent the forward direction. The key model parameters were set as follows: sun zenith angle $\theta_{s}=30^{\circ}$, relative azimuth angle between sun and view $\Psi=0^{\circ}$, chlorophyll content $C_{a b}$ $=40 \mu \mathrm{g} \mathrm{cm}^{-2}$, leaf structure parameters $N=1.5, \mathrm{LAI}=3$, leaf inclination parameters $\mathrm{LIDFa}=0.5$ and $\mathrm{LIDFb}=0.5$.

The SCOPE simulations and the spectral invariant theory support the relationship we derived. The relationship between $\sigma_{F C}$ and $R$ was developed analytically in section 2 . It was confirmed by SCOPE simulation. SCOPE has a fundamentally different basis of radiative transfer compared to the theory presented in section 2 . In the simulations, a wide range of scenarios were tested, including various canopy structure, leaf properties and sun zenith angles. Obviously, the validation is limited for canopies that can be represented by SCOPE, but the presented relationship is more general. Furthermore, the alternative approach to derive Eq. 3.12 by means of the widely used spectral invariant theory contributes to the confidence in our derivation.

\subsection{Implications for remote sensing}

The presented relationship between canopy scattering of SIF and reflectance has potential applications in remote sensing. The relationship we found can be used to correct the directional effect on SIF. Eq. 3.12 explains the similarity of the directional effects of SIF and of reflectance. The proportionality between $R$ and $\sigma_{F C}$ with the ratio (i.e., $i_{0} \omega$ in the far-red region) that is independent to view angles results the same directional effects 
of SIF and reflectance. Normalizing TOC SIF by reflectance may be an approach to correcting the viewing angle effects on SIF.

Eq. 3.12 also makes it possible to estimate the total emitted SIF using directional measured SIF and reflectance. A requirement is that the canopy interceptance and the leaf albedo are known. Canopy interceptance is a widely used spectral invariant that only depends on the canopy structure and sun position (Huang et al., 2007; Smolander and Stenberg, 2005). It can either be computed giving the canopy structure and sun zenith angle (Stenberg and Manninen, 2015), or estimated from canopy reflectance (Asrar et al., 1986; Bartlett et al., 1989). Leaf albedo in the far-red region is close to unity due to the strong scattering within the leaf. Nevertheless, it is necessary to estimate its value in order to apply Eq. 3.12, which means that inverse radiative transfer modelling may still be required in order to obtain $\omega$. Once $\omega$ and $i_{0}$ are known, canopy scattering of far-red SIF can be estimated from reflectance data, and the canopy structural and functional regulation on far-red SIF decoupled. Several studies interpret SIF observation from satellite by normalizing the cosine of solar zenith angle as a better indicator of plant physiology changes (Frankenberg et al., 2011; Joiner et al., 2011). This normalization accounts for the solar zenith angle dependence of the incoming solar irradiance, but does not account for spatial or temporal variations in fraction of absorbed PAR (FAPAR) and $\sigma_{F C}$ (Joiner et al., 2011). Cosine of solar zenith angle is related to $i_{0}$ (Stenberg and Manninen, 2015). Normalizing TOC SIF by cosine of solar zenith angle and TOC reflectance may partially correct the variation of $\sigma_{F C}$.

It is promising to consider the canopy structure effects on FAPAR together with $\sigma_{F C}$. Canopy structure effects on SIF observations include the impact on the process of light absorption and the process of scattering of emitted SIF. By 'removing' these effects from the observed SIF, we are able to estimate the fluorescence emission efficiency, which is a 'pure' functional trait directly linked to photosynthetic efficiency. The fluorescence emission efficiency can be computed as the normalization of SIF by the product of $\sigma_{F C}$ and absorbed PAR. In this study, we propose an approach for estimating 
canopy scattering of SIF from TOC reflectance, canopy interceptance and leaf albedo. The fraction of absorbed PAR can be also estimated by using leaf albedo and spectral invariants (Fan et al., 2014). Considering absorption of incident light and scattering of emitted light together, we may quantify the total effects of canopy structure on SIF observations only by using TOC reflectance.

\section{Conclusion}

Separation of canopy structural and photosynthesis functional regulation on SIF observations is crucial for estimating photosynthesis from SIF. The analogy of the radiative transfer of emitted SIF and intercepted incident light allows linking canopy scattering of SIF to reflectance observations. By comparing their radiative transfer, a theoretical relationship between reflectance and canopy scattering of SIF was found: Canopy scattering of far-red SIF can be expressed as a simple function of canopy interceptance, TOC reflectance and leaf albedo. The equation is valid if the directionality of leaf fluorescence emission is similar to the directionality of leaf scattering, a condition that is met in the far-red region, but not in the red region of the electromagnetic spectrum. The relationship we presented provides an easy and accurate approach for decoupling the canopy structural and directional effects on far-red SIF measurements. The relationship between canopy scattering of SIF and reflectance explicates that reflectance and SIF observations combined can provide a diagnose of photosynthetic functioning. 


\section{Canopy structure effects on SIF*}

*This chapter is based on: Yang, P., van der Tol, C., Verhoef, W., 2018. FCVI: A vegetation reflectance index for interpreting sun-induced chlorophyll fluorescence. Remote Sensing of Environment, under review. 


\section{Abstract}

Attempts of using sun-induced chlorophyll fluorescence (SIF) for tracking plant photosynthetic activity have been made in the past decades. SIF shows potential for improving estimation of gross primary productivity (GPP) and detecting plant stress. However, remote sensing of photosynthesis from SIF is still challenging because their relationship is superimposed by many other factors. It is particularly difficult to quantify and correct for the effects of canopy structure and sun-observer geometry on SIF. In this study, we propose a physically-based vegetation reflectance index to normalize SIF for these effects. The fluorescence correction vegetation index (FCVI) as a difference between near-infrared and broadband visible (400 - $700 \mathrm{~nm}$ ) reflectance quantifies the effects of light absorption and SIF re-absorption (and scattering) on observed SIF signals. Simulations from a radiative transfer model (SCOPE) reveal that FCVI estimates canopy structure and sun-observer geometry effects on near-infrared SIF with $R^{2}>0.98$. Normalizing SIF by FCVI and photosynthetically active radiation (PAR) results in the 'fluorescence emission efficiency' $\left(\epsilon_{F}\right)$ at photosynthetic level: a diagnosis of the energy distribution in photosystems. The FCVI derived from MODIS is further used to compute $\epsilon_{F}$ at the global scale at monthly time steps for the past 10 years from GOME-2 SIF measurements. The results show that the normalization is successful: retrieved $\epsilon_{F}$ is uncorrelated with NDVI $(r=-0.04)$, whereas SIF and NDVI are correlated $(r=0.64)$. Moreover, the response of normalized SIF from GOME-2 data to illumination intensity matches well with existing knowledge. These findings suggest that FCVI effectively separates canopy structure and plant functional traits from SIF signals. SIF and FCVI can be used together to assess the photosynthetic functioning of vegetation ecosystems. 


\section{Introduction}

Photosynthesis as one of the most fundamental processes on Earth provides all of the organic compounds and oxygen for humans and animals. Tracking photosynthesis from space is crucial for understanding climate change and ensuring the supply of food for the growing global population. Sun-induced chlorophyll fluorescence (SIF) is closely related to the light harvesting process of photosynthesis and responds nearly instantaneously to rapid changes in photosynthesis (Grace et al., 2007). Spatial and temporal measurements of SIF provide information about the gross primary productivity (GPP) of terrestrial ecosystems (Guanter et al., 2014; Migliavacca et al., 2017) and reveal plant stress (Ač et al., 2015; Rossini et al., 2015).

SIF can be measured from space using high spectral resolution and signal-to-noise ratio sensors covering Fraunhofer Lines in the fluorescence emission spectral range (i.e. 640 - $850 \mathrm{~nm}$ ) (Meroni et al., 2009). Global maps of SIF have been retrieved from radiance spectra measured by onboard spectrometer of the Greenhouse Gases Observing Satellite (GOSAT) (Joiner et al., 2011; Frankenberg et al., 2011) and of the Global Ozone Monitoring Experiment-2 satellite (GOME-2) (Joiner et al., 2013; Köhler et al., 2015; Khosravi et al., 2015; Guan et al., 2016), and of the Orbiting Carbon Observatory satellite (OCO-2) (Frankenberg et al., 2014; Sun et al., 2015). Sentinel 5P TROPOspheric Monitoring Instrument (TROPOMI) (Guanter et al., 2015) and TanSat (Xu et al., 2018) have the potential to measure SIF from space as well. Additionally, the FLuorescence EXplorer (FLEX) satellite mission has been selected as the 8th Earth Explorer mission of the European Space Agency (ESA). FLEX will be the first satellite mission dedicated to SIF observation, and will allow retrieving the full spectrum of fluorescence with high spatial resolution (Drusch et al., 2016).

The connection between fluorescence and photosynthesis has been explored by using leaf-level fluorescence measurements with the PAM technique (Baker, 2008). The quenching of fluorescence is a measure of photosynthetic efficiency (Genty et al., 1989). Temporally averaged satellite-based SIF data 
appear to correlate strongly with GPP (Frankenberg et al., 2011; Joiner et al., 2014; Guanter et al., 2014; Migliavacca et al., 2017). However, studies show that a substantial variability of SIF is due to canopy structure and sun-observer geometry (Porcar Castell et al., 2014; Van der Tol et al., 2016; Verrelst et al., 2016; Liu et al., 2016). It is explained by a conceptual formula for SIF measured by a sensor.

$$
\mathrm{SIF}=\frac{1}{\pi} \mathrm{PAR} \cdot \mathrm{PPAR} \cdot \epsilon_{F} \cdot \sigma_{F}
$$

The SIF observed is determined by three processes and each of the processes corresponds to one quantity in the above equation.

1. Absorption of incident photosynthetically active radiation (PAR) by chlorophylls in plants. The ratio between absorbed PAR by chlorophylls and incident PAR is fraction of absorbed PAR (fPAR).

2. Excitation of fluorescence in photosystems. The yield of fluorescence production to the absorbed PAR is fluorescence emission efficiency $\left(\epsilon_{F}\right.$, the subscript $F$ refers to fluorescence), and thus PAR.fPAR $\cdot \epsilon_{F}$ is the total emitted fluorescence radiation.

3. Scattering and re-absorption of the emitted SIF. The ratio between observed and emitted fluorescence radiation is scattering of SIF or escape probability of $\operatorname{SIF}\left(\sigma_{F}\right)$.

Among these processes, the emission of fluorescence reflects the oxidation status of the photosystems. The emission efficiency is inversely proportional to the sum of photochemical and non-photochemical quenching (Van der Tol et al., 2014). Its link with the light use efficiency of photosynthesis makes $\epsilon_{F}$ is a suitable indicator of plant stress (Ač et al., 2015). In contrast, the interactions between incident light and canopy, and between emitted SIF and canopy (i.e. the first and third process) are determined by canopy structure, leaf optical properties and sun-observer geometry. These two processes are related to the radiative transfer of the incident light and emitted SIF, and their product is called the 'radiative transfer factor' (i.e., $\left.\Gamma_{r t}=\mathrm{fPAR} \times \sigma_{F}\right)$. 
Simply normalizing SIF by absorbed PAR (APAR) has been regarded as the light use efficiency of fluorescence of the canopy (Daumard et al., 2012; Miao et al., 2018). This is a reasonable first order approximation. He et al. (2017) shows that angular normalization of SIF measurements is better linked with GPP than SIF itself. It suggests the needs of considering the sun-observer geometry. However, these cases only correct partially canopy structure and sun-observer geometry effects. Neither SIF/APAR nor angular normalization of SIF is sufficient to reveal the photosynthetic functioning.

Accurate quantification of both fPAR and $\sigma_{F}$ by using radiative transfer models (RTMs) requires knowledge of the canopy structure and leaf properties. RTMs simulate the light interaction with the canopy, including the absorption of incident light, and scattering and re-absorption of fluorescence emanated by photosystems. In remote sensing applications, the canopy structure and leaf properties are mostly unknown. It is possible to retrieve the required vegetation variables from top-of-canopy (TOC) reflectance measurements by using model inversion (Van der Tol et al., 2016). However, this procedure has several drawbacks: 1). Retrieval of vegetation parameters in most cases requires spectral reflectance in the whole optical region instead of just reflectance of a few bands; 2). Model inversion is usually ill-posed (Verrelst et al., 2014) and the accuracies of the vegetation variables retrieved are not guaranteed; 3). Using either look-up tables or numerical optimization for the retrieval process is time and computation consuming.

In our previous study (Yang and van der Tol, 2018), we analytically linked canopy scattering of SIF with TOC reflectance. The canopy scattering of far-red SIF is expressed as a function of TOC reflectance, leaf albedo and canopy interceptance (i.e., the portion of incident light will not go through the canopy directly). TOC reflectance is one of the most common remote sensing observations, but leaf albedo and canopy interceptance are not. Besides this limitation, the within-leaf scattering of SIF has not been considered in our previous study.

Accurate estimation of fPAR is also challenging. There are several approaches to estimate fPAR. The use of normalized difference vegetation 
index (NDVI) is a well-accepted approach (Myneni et al., 2002; Paruelo et al., 1997). Near-linear relations between NDVI and fPAR have been reported by many studies (Rouse Jr et al., 1974; Viña and Gitelson, 2005), because their functional response to leaf area, leaf orientation, sun zenith angle and atmospheric optical depth is similar (Myneni et al., 2002). However, the empirical parameters (or coefficients) for this approach are limited to specific cases (e.g. a regional area or at a certain period). Alternatively, fPAR can be estimated from TOC reflectance data by inverting a RTM, but this approach has the problems mentioned before. Fan et al. (2014) proposed an approach for fPAR modelling by using spectral invariants. This last approach is an interesting line to follow, but it still requires the leaf albedo, canopy interceptance as inputs, which are difficult to estimate.

In this study, we quantify the absorption of PAR, and the re-absorption and scattering of emitted fluorescence by using spectral invariant theory (Stenberg et al., 2016). We propose a physically-based model for fPAR, and a reflectance index to approximate canopy structure and sun-observer geometry effects on SIF $\left(\Gamma_{r t}\right)$ such that fluorescence emission efficiency $\left(\epsilon_{F}\right)$ can be estimated from SIF using only reflectance and SIF data. We first give the theoretical basis for fPAR model and the reflectance index FCVI (i.e. fluorescence correction vegetation index) and evaluate them by using simulations from a radiative transfer model SCOPE (Soil Canopy Observation, Photochemistry and Energy fluxes) (Van der Tol et al., 2009). Further evaluation is conducted by applying FCVI to GOME-2 SIF products. We use global maps of FCVI derived from MODIS reflectance products (Justice et al., 1998) to normalize GOME-2 SIF products for the effects of canopy structure and sun-observer geometry. Global maps of the fluorescence emission efficiency, which is directly linked to photosynthetic efficiency, are produced. The fluorescence emission efficiency data are also verified by comparing them with simulations from a biochemical model. 


\section{Theoretical basis}

The proposed fluorescence correction vegetation index (FCVI) for the radiative transfer factor is given by

$$
\mathrm{FCVI}=R_{n i r}-R_{v i s}
$$

where $R_{n i r}$ is the near-infrared (NIR) reflectance and $R_{v i s}$ is the broadband visible (VIS) reflectance (from 400 - $700 \mathrm{~nm}$ ).

The derivation of the index can be achieved with two steps: calculation of scattering of SIF $\left(\sigma_{F}\right)$ and calculation of fraction of absorbed photosynthetically active radiation (fPAR). In our previous study (Yang and van der Tol, 2018), we derived the relationship between canopy scattering of far-red SIF $\left(\sigma_{F C}\right)$ and TOC reflectance analytically, by investigating the radiative transfer of incident light and emitted SIF. In the following, we first briefly explain the relationship by using the spectral invariant theory (Stenberg et al., 2016) and include the within-leaf scattering of SIF. Further, we give the theoretical basis for our fPAR model and for FCVI.

\subsection{A short review of spectral invariant theory}

The spectral invariant approach allows for a very accurate parametrization of the canopy scattering, absorption, TOC directional reflectance by using the wavelength dependent leaf albedo $\omega$ (i.e., the sum of leaf reflectance and transmittance) and three spectrally invariant and structurally varying parameters: canopy interceptance $\left(i_{0}\right)$, recollision probability $(p)$ and directional escape probability $(\rho(\Omega), \Omega$ indicates the viewing direction).

The theory describes radiative transfer of incident photons with canopies in a simple way. Photons coming from the top of a canopy will either go through the canopy via gaps or interact with leaves (or needles). A portion of the photons is intercepted by the canopy and this portion is defined as the canopy interceptance $\left(i_{0}\right)$ (Smolander and Stenberg, 2005; Schull et al., 2007; Knyazikhin et al., 2013). As a result of an interaction, photons can either be scattered or absorbed by a leaf, depending on the leaf (or needle) albedo. The scattered photons interact with leaves in the canopy again with a probability. 
The probability that a photon, after having survived an interaction with a canopy element, will interact with the canopy again is called the recollision probability $(p)$. Smolander and Stenberg (2005) expressed absorption $a(\lambda)$ and total scattering $s(\lambda)$ of a canopy at wavelength of $\lambda$ (bounded underneath by a non-reflecting surface).

$$
\begin{aligned}
& a(\lambda)=i_{0} \frac{1-\omega(\lambda)}{1-p \omega(\lambda)} \\
& s(\lambda)=i_{0} \frac{(1-p) \omega(\lambda)}{1-p \omega(\lambda)}
\end{aligned}
$$

The scattered photons can escape the vegetation canopy through various directional paths including the upper and lower boundary. Remote sensing normally detects signals from one direction above the upper boundary of canopy. In order to predict the directional signals, another spectral invariant, called directional escape probability $(\rho(\Omega)$ ), was introduced (Huang et al., 2007). The portion of scattered photons that escapes via gaps in the direction of viewing is $\rho(\Omega)$, the directional escape probability. Canopy directional reflectance can be expressed as

$$
R=i_{0} \rho(\Omega) \frac{\omega(\lambda)}{1-p \omega(\lambda)}
$$

\subsection{Linking SIF scattering with reflectance}

Canopy scattering of SIF can be expressed by using the spectral invariant theory as well (Yang and van der Tol, 2018). A TOC SIF observation includes contributions from: 1) emitted SIF photons from leaves that directly escape via $\rho(\Omega) ; 2$ ) emitted SIF photons that interact with leaves in the canopy again (one order) and escape and are observed via $p \omega(\lambda) \rho(\Omega) ; 3$ ) the scattered SIF photons have the second interaction and escape and are observed via $p^{2} \omega(\lambda)^{2} \rho(\Omega)$, etc. The total canopy scattering of SIF is the sum of the contributions, and is given as a geometric series

$$
\sigma_{F C}(\lambda)=\rho(\Omega)+p \omega(\lambda) \rho(\Omega)+p^{2} \omega(\lambda)^{2} \rho(\Omega)+\ldots=\frac{\rho(\Omega)}{1-p \omega(\lambda)}
$$


Comparing Eq. 4.5 and Eq. 4.6, we obtain the relationship.

$\sigma_{F C}(\lambda)=\frac{R(\lambda)}{i_{0} \omega(\lambda)}$

It is important to note that spectral invariant theory ignores the difference between leaf reflectance and transmittance $\left(\rho_{l}-\tau_{l}\right)$, and only uses the sum of them $\left(\rho_{l}+\tau_{l}=\omega\right)$ for the scattering of a leaf. The simplification is reasonable in the near-infrared region, but not in the visible region. For a more generic derivation of the relationship (Eq. 4.7), the reader is referred to Yang and van der Tol (2018).

The total scattering of SIF includes scattering in a canopy $\left(\sigma_{F C}\right)$ disscued above and the scattering within a leaf $\left(\sigma_{F L}\right)$. The scattering of SIF within the leaf may be approximated by the leaf albedo. We obtain the scattering of SIF including within-leaf scattering

$$
\sigma_{F}(\lambda)=\sigma_{F C} \times \sigma_{F L}=\frac{R(\lambda)}{i_{0}}
$$

\subsection{Estimating fPAR from reflectance}

The fraction of absorbed photosynthetically active radiation fPAR is the fraction of incoming solar radiation that is absorbed by vegetation in the spectral range from 400 to $700 \mathrm{~nm}$. By definition, fPAR the fraction of absorbed PAR and can be expressed as

$$
\mathrm{fPAR}=\frac{\int_{400-700 \mathrm{~nm}} a(\lambda) E(\lambda) d \lambda}{\int_{400-700 \mathrm{~nm}} E(\lambda) d \lambda}
$$

where $E$ is the incident irradiance, and $\lambda$ is the wavelength.

In order to using reflectance to estimate fPAR, we express canopy absorption as a function of reflectance by comparing Eq. 4.5 and Eq. 4.3.

$$
a(\lambda)=i_{0}-\frac{1-p}{\rho(\Omega)} R(\lambda)
$$

The term after the minus sign equals to the canopy scattering $(s)$, and this equation is equivalent to $a(\lambda)=i_{0}-s(\lambda) .1-p$ and $\rho(\Omega)$ are the probabilities of photons survived an interaction, will escape the canopy from all directions and from the viewing direction $(\Omega)$, respectively. 
Introducing Eq. 4.10 into Eq. 4.9 yields

$$
\begin{aligned}
\mathrm{fPAR} & =i_{0}-\frac{1-p}{\rho(\Omega)} \cdot \frac{\int_{400-700 \mathrm{~nm}} R(\lambda) E(\lambda) d \lambda}{\int_{400-700 \mathrm{~nm}} E(\lambda) d \lambda} \\
& =i_{0}-\frac{1-p}{\rho(\Omega)} R_{v i s}
\end{aligned}
$$

where $R_{v i s}$ is the broadband visible reflectance ranging from 400 to $700 \mathrm{~nm}$.

We notice that the quantity $\frac{i_{0} \rho(\Omega)}{1-p}$ is the so called directional area scattering factor $\left(\mathrm{DASF}=\frac{i_{0} \rho(\Omega)}{1-p}\right)$, which is a parameter that describes reflectance of nonabsorbing canopies, and is independent of the choice of leaf albedo (Knyazikhin et al., 2013). Using this definition, Eq. 4.11 can be written as

$$
\mathrm{fPAR}=i_{0}\left[1-\frac{R_{v i s}}{\mathrm{DASF}}\right]
$$

DASF is almost proportional to and may be approximated by NIR reflectance (Knyazikhin et al., 2013). From Eq. 3.15, we obtain

$$
1 / R_{n i r}=\frac{1}{\mathrm{DASF}}+\frac{1-\omega_{n i r}}{i_{0} \rho(\Omega) \omega_{n i r}}
$$

Combining Eq. 4.12 and Eq. 4.13, we obtain

$$
\mathrm{fPAR}=i_{0} \frac{R_{n i r}-R_{v i s}}{R_{n i r}}+\frac{R_{v i s}\left(1-\omega_{n i r}\right)}{\rho(\Omega) \omega_{n i r}}
$$

In the NIR spectral region, because both $R_{v i s}$ and (1- $\left.\omega\right)$ are small values, the term after the plus sign is negligible. Finally, fPAR can be expressed as

$$
\mathrm{fPAR}=i_{0} \frac{R_{n i r}-R_{v i s}}{R_{n i r}}
$$

This new fPAR model is similar to NDVI (i.e. $\frac{R_{n i r}-R v i s}{R_{n i r}+R v i s}$ ) in term of the use of NIR and VIS reflectance, but our model requires canopy interceptance as an input. 


\subsection{Reflectance index for the radiative transfer factor}

Both the scattering of SIF and fPAR are approximated as a function of canopy directional reflectance and the canopy interceptance. The radiative transfer factor of SIF, as a product of them, are further expressed as

$$
\Gamma_{r t}=\mathrm{fPAR} \times \sigma_{F}=R_{n i r}-R_{v i s}
$$

The radiative transfer factor is simply approximated by the difference of NIR reflectance and broadband VIS reflectance. Note that the unknown $i_{0}$ is eliminated from this equation.

\section{Evaluation of fPAR model and FCVI}

We evaluate our fPAR model, and the relationship between canopy structure and sun-observer geometry effects of SIF $\left(\Gamma_{r t}\right)$ and reflectance $\left(R_{n i r}-R_{v i s}\right)$ as shown in Eq. 4.16 by using simulations from the SCOPE model (Van der Tol et al., 2009) for a number of scenarios. Further, we apply FCVI to the GOME-2 SIF data to estimate fluorescence emission efficiency. We evaluate the index by means of analysing the resulted fluorescence emission efficiency data.

\subsection{Evaluation by using simulations from SCOPE model}

\subsubsection{Model description}

SCOPE is a model for homogeneous vegetation and consists of a leaf RTM, three canopy RTMs and an energy balance model. At the leaf level, Fluspect (Vilfan et al., 2016), which is based on PROSPECT (Jacquemoud and Baret, 1990), simulates leaf reflectance, transmittance, and fluorescence emission of the both forward and backward side. At the canopy level, RTMo and RTMf, which are two SAIL (Scattering by Arbitrarily Inclined Leaves) based models (Verhoef, 1984), compute the radiative transfer of incident radiation and emitted fluorescence, respectively (i.e., the letter 'o' in RTMo refers to optics and 'f' in RTMf refers to fluorescence).

RTMo calculates the fate of incident irradiance and absorbed radiation of each leaf within the canopy. It provides the TOC reflectance simulations, 
and absorbed PAR (APAR) of each leaf orientation class in different position of the canopy. The number of leaf orientation classes considered is 468, comprising the combinations of 13 leaf's normal zenith angles and 36 leaf's azimuths with respect to the sun. The leaf inclination distribution is parametrized with two $(\mathrm{a}, \mathrm{b})$ parameters, which control the mean leaf zenith angle and the bimodality of the distribution (Verhoef, 1984).

The energy balance model predicts temperature of each leaf in the canopy. The vegetation RTMs and canopy energy balance model interact with each other, and update leaf temperature prediction. The calculated absorbed (PAR) radiation and leaf temperature are required for simulating the fluorescence emission efficiency in a biochemical model (Van der Tol et al., 2014). Further, RTMf simulates the radiative transfer of emitted fluorescence to obtain the TOC fluorescence.

\subsubsection{Database generation and simulation method}

We first generated 10800 scenarios. The input of SCOPE for the scenarios comprises 60 combinations of leaf properties, 15 combinations of canopy structure parameters, and 12 combinations of sun-observer geometry (Table 4.1). The values of parameters for leaf properties, canopy structure and sun position were chosen within the recommended ranges in SCOPE (Van der Tol et al., 2009; Yang et al., 2017) with non-linear steps. LIDFa determined the average leaf angle (i.e., $\mathrm{ALA}^{\circ}=45-\frac{360}{\pi^{2}} \mathrm{LIDFa}$ ) (Verhoef, 1998). The combinations of sun-observer geometry not only include nadir observations (i.e., $\theta_{o}=0^{\circ}$ ), but also the observations in the hot spot (i.e., $\theta_{o}=\theta_{s}=30^{\circ}$ and $\psi=0^{\circ}$ ) and cold spot direction (i.e., $\theta_{o}=\theta_{s}=30^{\circ}$ and $\psi=180^{\circ}$ ). A typical incident irradiance spectrum was used in the scenarios (see supplementary materials). In the theoretical derivation, non-reflecting soil background is assumed. To evaluate the soil effects on the performance of the fPAR model and FCVI, simulations of the 10800 scenarios were conducted both on a non-reflecting background (i.e., soil reflectance was set to zero) and on a real soil background (i.e., for the reflectance see supplementary materials). 
Table 4.1: Summary of SCOPE inputs applied for the generation of the database

\begin{tabular}{llll}
\hline Parameter & Explanation & Unit & Values \\
\hline$C_{a b}$ & chlorophyll $a+b$ content & $\mu \mathrm{g} \mathrm{cm}^{-2}$ & $5,10,20,40,80$ \\
$C_{d m}$ & leaf mass per unit area & $\mathrm{g} \mathrm{cm}^{-2}$ & $0.01,0.02$ \\
$C_{w}$ & equivalent water thickness & $\mathrm{cm}$ & $0.015,0.03$ \\
$N$ & leaf structure parameter & - & $1,1.5,2$ \\
LAI & leaf area index & - & $0.5,1,2,3,6$ \\
LIDFa & leaf inclination function parameter a & - & $-1,0,1$ \\
$\theta_{s}$ & sun zenith angle & $\circ$ & $30,45,60$ \\
$\theta_{o}$ & viewing zenith angle & $\circ$ & 0,30 \\
$\psi$ & difference between sun and viewing azimuth angle & $\circ$ & 0,180 \\
\hline
\end{tabular}

We then simulated TOC directional SIF and TOC directional reflectance for each scenario. In the simulations, $\epsilon_{F}\left(\mathrm{~nm}^{-1}\right)$ was given as shown in Fig. 4.1. Its integration over the whole spectral region results in fluorescence emission efficiency of photosystem I and II, which was 0.024 . The values of $\epsilon_{F}$ at $740 \mathrm{~nm}$ and at $760 \mathrm{~nm}$ were $8.51 \times 10^{-5} \mathrm{~nm}^{-1}$ and $5.03 \times 10^{-5} \mathrm{~nm}^{-1}$, respectively. Besides fluorescence emission efficiency, PAR was also kept constant at $175 \mathrm{~W} \mathrm{~m}^{-2}$. Both fluorescence emission efficiency and incident PAR are required as model inputs, but are not relevant to $\Gamma_{r t}$ which is only determined by the canopy structure, leaf optical properties and sun-observer geometry.

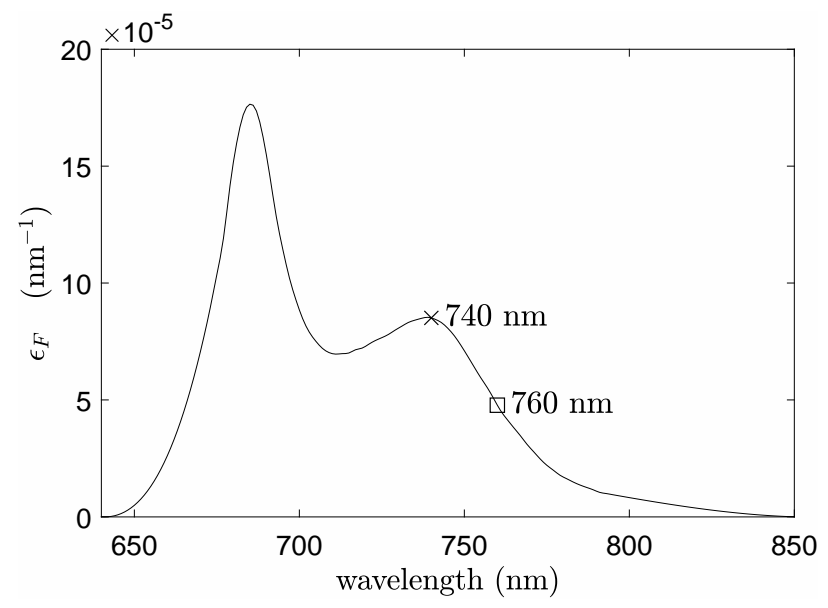

Figure 4.1: Fluorescence emission efficiency used as input of the SCOPE model in the spectral region from $640 \mathrm{~nm}$ to $850 \mathrm{~nm}$. The markers indicate locations of $740 \mathrm{~nm}$ and $760 \mathrm{~nm}$. 


\subsubsection{Evaluation of the new fPAR model}

We first evaluate the fPAR model by using the SCOPE simulations. The model we developed as shown in Eq. 4.15 requires canopy interceptance $\left(i_{0}\right)$, NIR and broadband VIS reflectance as inputs. The fraction of absorbed photosynthetically active radiation (fPAR) is computed by SCOPE and stored directly as an output for the 10800 scenarios. Canopy interceptance $\left(i_{0}\right)$ is calculated as $1-\exp (-k L)$, where $k$ is the extinction coefficient and $L$ is canopy leaf area index (Stenberg and Manninen, 2015; Yang and van der Tol, 2018). The extinction coefficient is determined by sun zenith angle and leaf inclination distribution. $i_{0}$ is computed by SCOPE directly, but it is not stored as an output. We modified SCOPE version 1.70 to store it as an output.

We compared the performance of the new model to NDVI. NDVI has been used as a semi-empirical reflectance index for estimating PPAR for decades. In this study, we calculated NDVI of the scenarios as $\frac{R_{n i r}-R v i s}{R_{n i r}+R v i s}$ by using the simulated TOC reflectance. We compared NDVI and our model with fPAR for the 10800 scenarios in order to benchmark the performance of our model.

\subsubsection{Evaluation of FCVI}

We evaluate the index FCVI for the radiative transfer factor $\left(\Gamma_{r t}\right)$ at $740 \mathrm{~nm}$ and at $760 \mathrm{~nm}$. The two wavelengths were chosen for meeting the needs of remote sensing applications. The retrieval of SIF relies on the solar or telluric atmospheric absorption lines (also called Fraunhofer Lines) (Meroni et al., 2009). The two $\mathrm{O}_{2}$ absorption features at $687 \mathrm{~nm}$ and $760 \mathrm{~nm}$ have been widely used in SIF retrieval. The wavelength of $680 \mathrm{~nm}$ was not tested because the index is not applicable at this wavelength. In Yang and van der Tol (2018), we have discussed that there was not a simple relationship between canopy scattering of red SIF $(687 \mathrm{~nm})$ and TOC reflectance. The index was tested at wavelength of $740 \mathrm{~nm}$ as a preparation for the further application to GOME-2 SIF products, which is at $740 \mathrm{~nm}$. 
FCVI of the scenarios was simply calculated as the difference between NIR and broadband VIS reflectance as shown in Eq. 3.12. In practice, the average value of visible reflectance at each wavelength can be used, because the difference between average and broadband VIS reflectance is rather small and negligible. For instance, the maximum difference between average VIS reflectance and broadband VIS reflectance of the 10800 scenarios simulated by SCOPE was 4.1\%. For NIR reflectance, we took the reflectance at $770 \mathrm{~nm}$ mainly considering the data availability in the remote sensing campaigns and missions. This reflectance is normally available in campaigns or satellites dedicated to SIF measurements and yet not too close to the absorption feature at $760 \mathrm{~nm}$, where SIF has an apparent enhancement on reflectance. Reflectance from $750 \mathrm{~nm}$ to $900 \mathrm{~nm}$ may be used in practice, because vegetation NIR reflectance changes slightly from $750 \mathrm{~nm}$ to $900 \mathrm{~nm}$. For example, the largest variation of reflectance in this spectral region of the scenarios as simulated by SCOPE was less than 0.1.

$\Gamma_{r t}$ was computed from simulated TOC observed SIF, $L_{o}^{F}\left(\mathrm{~W} \mathrm{~m}^{-2}\right.$ $\mathrm{nm}^{-1} \mathrm{sr}^{-1}$ ) of the scenarios and several inputs, notably, incident PAR (W $\mathrm{m}^{-2}$ ) and fluorescence emission efficiency at the studied wavelengths, $\epsilon_{F}$ $\left(\mathrm{nm}^{-1}\right)$. It is expressed as

$$
\Gamma_{r t}=\frac{\pi L_{o}^{F}}{\mathrm{PAR} \cdot \epsilon_{F}}
$$

After obtaining $\Gamma_{r t}$ and FCVI of the scenarios, we studied the sensitivity of $\Gamma_{r t}$ to increasing LAI in the spectral region of $640 \mathrm{~nm}-850 \mathrm{~nm}$, and to change of various vegetation properties at $760 \mathrm{~nm}$. A 'base' scenario was assigned: $\mathrm{LAI}=3$, leaf chlorophyll $C_{a b}=40 \mu \mathrm{g} \mathrm{cm}{ }^{-2}$, leaf structure parameter $N=1.5$, leaf mass $C_{d m}=0.01 \mathrm{~g} \mathrm{~cm}^{-2}$, equivalent water thickness $C_{w}$ $=0.015 \mathrm{~cm}, \mathrm{LIDFa}=0$, sun zenith angle $\theta_{s}=30^{\circ}$ and viewing zenith angle $\theta_{o}=0^{\circ}$. In these analysis, a non-reflecting soil background was used. Finally, the simulated fPAR was compared with our fPAR model, and the simulated $\Gamma_{r t}$ was compared with FCVI for all the scenarios. Linear regressions were used to analyse their relation. The effects of background (i.e., non-reflecting and reflecting surface) were examined. 


\subsection{Evaluation by using GOME-2 SIF data}

We further evaluate FCVI by applying to GOME-2 SIF data and analysing the resulted fluorescence emission efficiency. The efficiency was calculated as:

$$
\epsilon_{F}=\frac{\pi \mathrm{SIF}_{\mathrm{GOME}}}{\mathrm{PAR} \cdot \mathrm{FCVI}}
$$

Note the retrieved fluorescence emission efficiency was only in one band since the SIF observations were at one band (i.e. $740 \mathrm{~nm}$ ).

\subsubsection{Satellite data}

The global SIF data were retrieved from GOME-2 level 1B data produced by Joiner et al. (2013) (http://avdc.gsfc.nasa.gov). The GOME2 spectrometer measures the Earth's backscattered radiance with a high spectral resolution (around $0.5 \mathrm{~nm}$ from 240 - $790 \mathrm{~nm}$ ) and high signal-tonoise ratio. The overpass time of GOME-2 is 9:30 (local time) (Joiner et al. 2013). It has a viewing swath width of $1920 \mathrm{~km}$ (Munro et al., 2016). The GOME-2 SIF products include level 2 (orbital) and level 3 (weekly and monthly) starting from 2007. We took the monthly averaged GOME-2 SIF (version 2.6) observations from the MetOp-A satellite at $740 \mathrm{~nm}$ in the past 10 years $(2007$ - 2016). The monthly averaged data has been gridded to a spatial resolution of $0.5^{\circ} \times 0.5^{\circ}$.

Atmospherically corrected surface reflectance data of MODerate resolution Imaging Spectroradiometer (MODIS) on the NASA Earth Observing System (EOS) Terra satellite were used to calculate FCVI instead of reflectance from GOME-2, because the reflectance from GOME-2 data are not atmospheric corrected. The standard MOD09 reflectance products, which is not BRDF (bidirectional reflectance distribution function) adjusted, were used. The overpass time of Terra is approximately at 10:30 (local time). It has a viewing swath width of $2330 \mathrm{~km}$ (Justice et al., 2002). Terra products were used to minimize the mismatches of sun-observer geometry between MODIS reflectance and GOME-2 SIF observations caused by their different overpass time and swath width. We took the MODIS reflectance observation at bands 
1, 2, 3, 4 (centred at $648 \mathrm{~nm}, 858 \mathrm{~nm}, 470 \mathrm{~nm}$ and $555 \mathrm{~nm}$ ). The averaged reflectance at bands 1, 3 and 4 was used for representing the broadband VIS reflectance, and reflectance at band 2 was used for representing NIR reflectance. The MODIS measurements were re-sampled to 0.5 by 0.5 degree and monthly averaged for consistency with the GOME-2 SIF data. Several thresholds were applied to filter non-vegetation pixels. We used NDVI $>0$ and SIF $>0$ for both GOME-2 SIF, PAR, and NDVI data.

\subsubsection{PAR calculation}

The bottom of atmosphere (BOA) PAR for a clear sky was estimated by using a simple analytical formula proposed by Frouin et al. (1989).

$$
\begin{aligned}
\mathrm{PAR}= & \mathrm{PAR}_{0} \cos \theta_{s} \\
& \cdot \frac{\exp \left[-(a+b / V) / \cos \theta_{s}\right]}{1-\bar{r}\left(a^{\prime}+b^{\prime} / V\right)} \\
& \cdot \exp \left[-a_{v}\left(U_{v} / \cos \theta_{s}\right)^{b_{v}}\right] \\
& \cdot \exp \left[-a_{o}\left(U_{o} / \cos \theta_{s}\right)^{b_{o}}\right] .
\end{aligned}
$$

Where $\mathrm{PAR}_{0}$ is the monochromatic extraterrestrial solar PAR $\left(\mathrm{Wm}^{-2}\right)$ and $\theta_{s}$ is the sun zenith angle. This formula accounts for the most important processes occurring within the atmosphere: scattering and absorption by molecules and aerosols; absorption by water vapor; absorption by ozone, corresponding to the three terms in Eq. 4.19. $V, U_{v}$ and $U_{o}$ refer to visibility, total water vapor and total ozone amount, respectively. $\cos \theta_{s}$ is one of the auxiliary data included in GOME-2 SIF products. $\bar{r}$ is the average reflectance in the PAR region. $a, b, a^{\prime}, b^{\prime}, a_{v}, b_{v}, a_{o}$ and $b_{o}$ are fitting coefficients. The values in the equation are shown in Table 4.2 as recommended in Frouin et al. (1989). These coefficients were kept constant for all the data, and only sun zenith angles derived the variation of TOC canopy incident PAR. This was a first order approximation, and for advanced use of the index, it may be improved by using more accurate incident PAR data. 
Table 4.2: Parameters used in the analytical estimation of bottom of atmosphere PAR.

\begin{tabular}{ccccccc}
\hline $\mathrm{PAR}_{0} \mathrm{Wm}^{-2}$ & $V \mathrm{~km}$ & $U_{v} \mathrm{gcm}^{-2}$ & $U_{o}$ atm $\mathrm{cm}$ & $\bar{r}$ & $a$ & $b$ \\
400 & 23 & 1.4 & 0.34 & 0.1 & 0.078 & 0.882 \\
\hline & $a^{\prime}$ & $b^{\prime}$ & $a_{v}$ & $b_{v}$ & $a_{o}$ & $b_{o}$ \\
& 0.123 & 0.594 & 0.002 & 0.87 & 0.052 & 0.99 \\
\hline
\end{tabular}

\subsubsection{Evaluation of normalized GOME-2 SIF}

We evaluated the fluorescence emission efficiency maps in several ways. We first examined the spatial pattern of the fluorescence emission efficiency maps in comparison to NDVI, SIF and PAR data. The correlations between each two of SIF, NDVI, $\epsilon_{F}$ and PAR were calculated and compared. Further, we investigated the correlation between incident light intensity (PAR) and $\epsilon_{F}$ retrieved from GOME-2 SIF. The 'light response' of $\epsilon_{F}$ retrieved from satellite data was compared with the simulated response from the biochemical model of Van der Tol et al. (2014).

We obtained the relationship between light intensity and the then years of fluorescence emission efficiency from GOME-2 SIF data. The range of incident PAR ( 0 to $350 \mathrm{~W} \mathrm{~m}^{-2}$ ) was binned with a interval of $8.7 \mathrm{~W}$ $\mathrm{m}^{-2}\left(50 \mu \mathrm{mol} \mathrm{m} \mathrm{m}^{-2} \mathrm{~s}^{-1}\right)$. Further, the average values for the pixels in given illumination conditions were computed and analysed with the PAR. Because few SIF measurements were available at low light intensity $\left(<50 \mathrm{~W} \mathrm{~m}^{-2}\right)$, the response of $\epsilon_{F}$ to the low light was not investigated from the satellite data.

Van der Tol et al. (2014) model is a photosynthetic energy distribution model, and is based on the conventional Farquhar et al. (1980) and Collatz et al. (1992) model. It simulates the efficiency of fluorescence emission, photochemistry and heat dissipation. The efficiencies are determined by (1) absorbed PAR (APAR), (2) leaf temperature, (3) the maximum rate of carboxylation $\left(V_{c \max }\right)$, and (4) other ambient environmental conditions, such as air pressure and concentrations of $\mathrm{O}_{2}$ and $\mathrm{CO}_{2}$ in the leaf boundary layer.

Model simulations were used to provide general knowledge of the light response of $\epsilon_{F}$. In the then years of global fluorescence emission efficiency 
data, vegetation types, environmental conditions covered almost all the possible cases on Earth. Corresponding to the biochemical model, it means that apart from the illumination condition, $\epsilon_{F}$ was also regulated by other environmental conditions (e.g., temperature, $\mathrm{CO}_{2}$ concentration) and also the plant functional traits (e.g., the maximum rate of carboxylation $V_{c m a x}$, photosynthetic pathways) that all vary between pixels and among months and years. We only presented the case of $V_{\text {cmax }}$ was 20, 60 and $100 \mu \mathrm{mol}$ $\mathrm{m}^{-2} \mathrm{~s}^{-1}$.

\section{Results}

\subsection{Performance of the new fPAR model}

There is a positive correlation between NDVI and fPAR with $r=0.88$ and $p<0.001$ for the scenarios with real soil. A simple linear regression is applied to predict fPAR based on NDVI. A significant regression equation is found fPAR $=1.16 \mathrm{NDVI}-0.07$ for the 10800 scenarios bounded underneath by a real soil surface, with an $R^{2}$ of 0.66 (Fig. 4.2). NDVI performs poorly in the 10800 scenarios bounded underneath by a non-reflecting surface. RMSE is 0.41 and $R^{2}$ is only 0.20 . In these cases, the values of NDVI range from 0.35 to 1 , whereas the values of fPAR range wider from 0.15 to 1 .

The model we proposed performs much better than NDVI in estimating fPAR among the simulated scenarios (Fig. 4.2). They are also positively correlated, but with a higher correlation $(r=0.98, p<0.001)$. Regression equations are found $\mathrm{fPAR}=0.94 i_{0} \frac{R_{n i r}-R_{v i s}}{R_{n i r}}+0.12$ for the scenarios bounded underneath by a real soil surface, with an $R^{2}$ of 0.98 , and fPAR $=1.06 i_{0} \frac{R_{n i r}-R_{v i s}}{R_{n i r}}+0.02$ for the scenarios bounded underneath by a nonreflecting surface. The fPAR values of the scenarios with non-reflecting surface are better predicted by our model than those with a reflecting surface as indicated in RMSEs (0.06 and 0.25). For both simulations of reflecting and non-reflectance scenarios, all the data are slightly above the 1-to-1 line. 

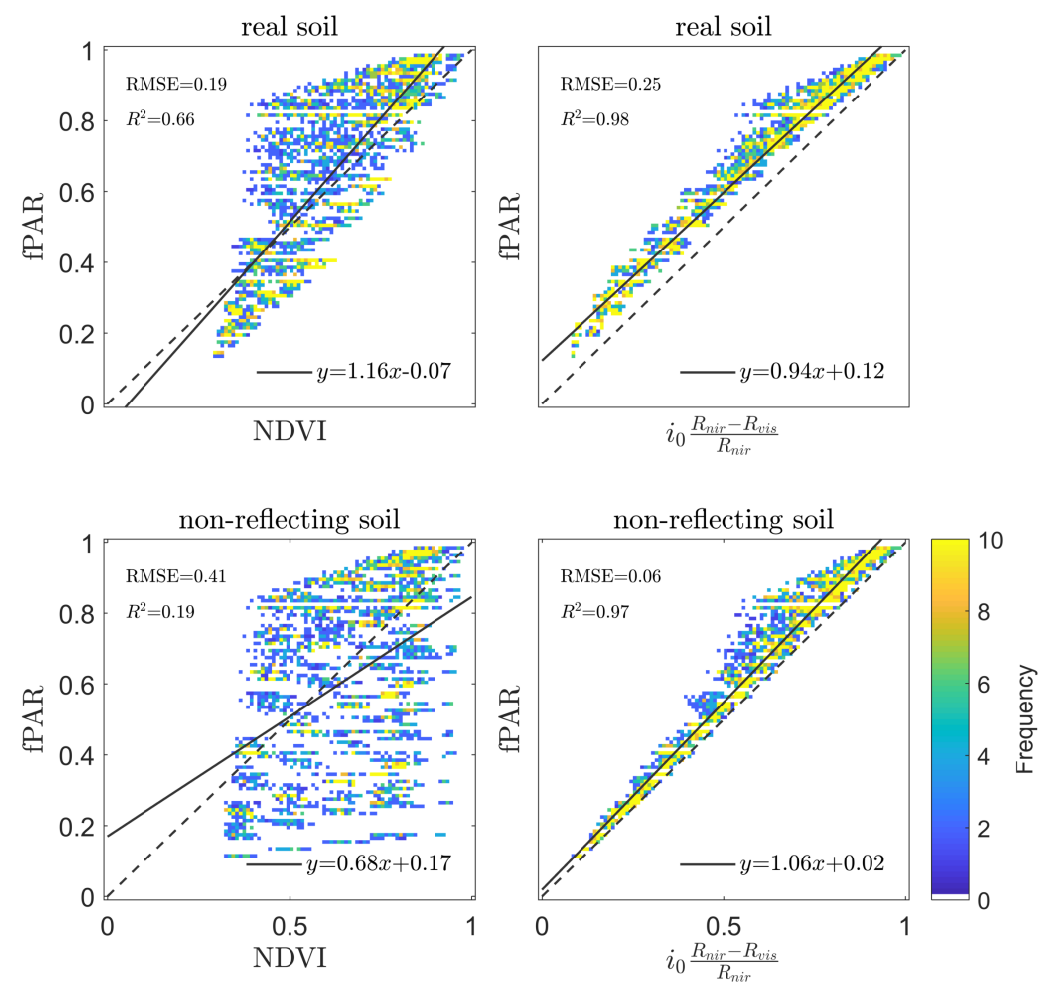

Figure 4.2: Comparison between NDVI and fPAR, and between $i_{0} \frac{R_{n i r}-R_{v i s}}{R_{n i r}}$ and fPAR for the 10800 scenarios with real soil and non-reflecting soil. The colours represent the number of scenario in a 0.01 by 0.01 grid.

\subsection{Radiative transfer factor}

The radiative transfer factor is spectrally dependent (Fig. 4.3). The spectra of $\Gamma_{r t}$ are similar to vegetation reflectance with lower values in the red region and much higher values in the NIR region. FCVI values (i.e. the dashed lines in Fig. 4.3) are similar to those of $\Gamma_{r t}$ in the near-infrared region, and obviously not in the red region. Both $\Gamma_{r t}$ and the difference between $\Gamma_{r t}$ and FCVI in the near infrared increase with LAI.

Fig. 4.4 shows that $\Gamma_{r t}$ at $760 \mathrm{~nm}$ is more sensitive to canopy structure (ALA and LAI) than to leaf properties. The non-linear change of $\Gamma_{r t}$ to LAI, as well as to ALA is shown in the sensitivity analysis. $\Gamma_{r t}$ is sensitive to leaf chlorophyll content, especially when the chlorophyll content is low. 


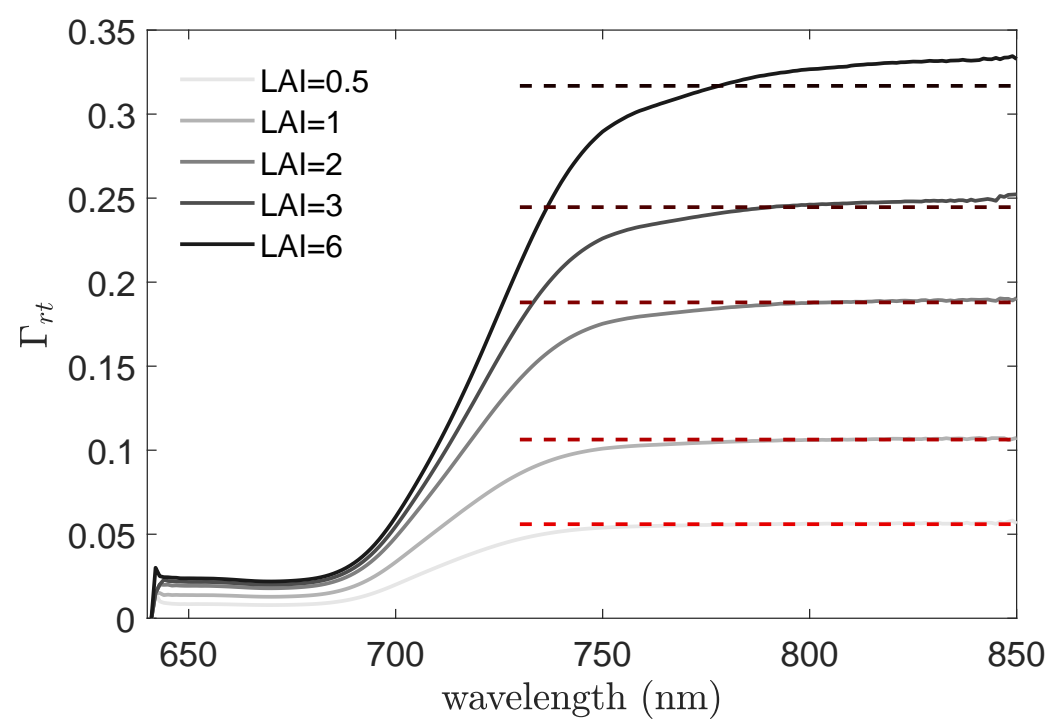

Figure 4.3: The radiative transfer factor of fluorescence $\left(\Gamma_{r t}=\mathrm{fPAR} \times \sigma_{F}\right)$ in the spectral region from $640 \mathrm{~nm}$ to $850 \mathrm{~nm}$ simulated by using SCOPE. The dashed lines are the values of FCVI for the five scenarios bounded underneath by non-reflecting surface.
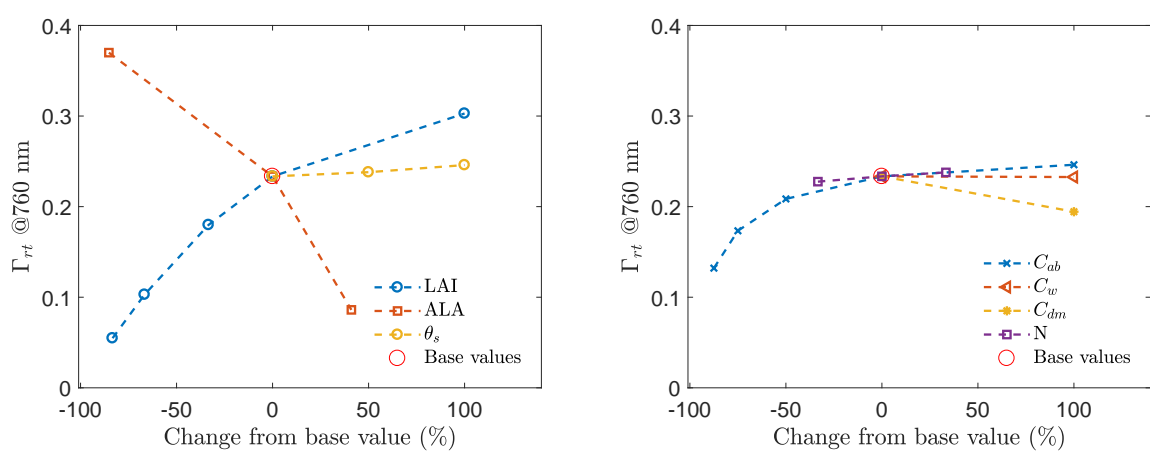

Figure 4.4: Spider plot for sensitivity analysis of the radiative transfer factor of SIF $\left(\Gamma_{r t}\right)$ at $760 \mathrm{~nm}$. The base values were set as follows. LAI $=3$. Leaf chlorophyll $C_{a b}=40 \mu \mathrm{g} \mathrm{cm}{ }^{-2}$. Leaf structure parameter $N=1.5$. Leaf mass $C_{d m}=0.01 \mathrm{~g} \mathrm{~cm}^{-2}$. Equivalent water thickness $C_{w}=0.015 \mathrm{~cm}, \mathrm{LIDFa}=0$ Sun zenith angle $\theta_{s}=30^{\circ}$. viewing zenith angle $\theta_{s}=0^{\circ}$.

\subsection{Performance of FCVI}

Fig. 4.5 shows that the reflectance index FCVI appears to be a good predictor of $\Gamma_{r t}$ at both $760 \mathrm{~nm}$ and $740 \mathrm{~nm}$. The values of most $\Gamma_{r t}$ at both wavelengths range from 0 to 0.5 . The correlation between FCVI and 
$\Gamma_{r t}$ is $0.96(p<0.001)$ at $760 \mathrm{~nm}$ and $0.97(p<0.001)$ at $740 \mathrm{~nm}$ for both non-reflecting and reflecting surface. FCVI slightly underestimates $\Gamma_{r t}$ in most scenarios when the real soil is used. When $\Gamma_{r t}$ is close to 0, FCVI is also close to 0 in the simulations with non-reflecting soil, whereas the values of FCVI are from 0.07 to 0.1 in those with real soil. According to RMSE, FCVI performs better when non-reflecting soil is used, but the performance is still satisfactory when real soil is used.
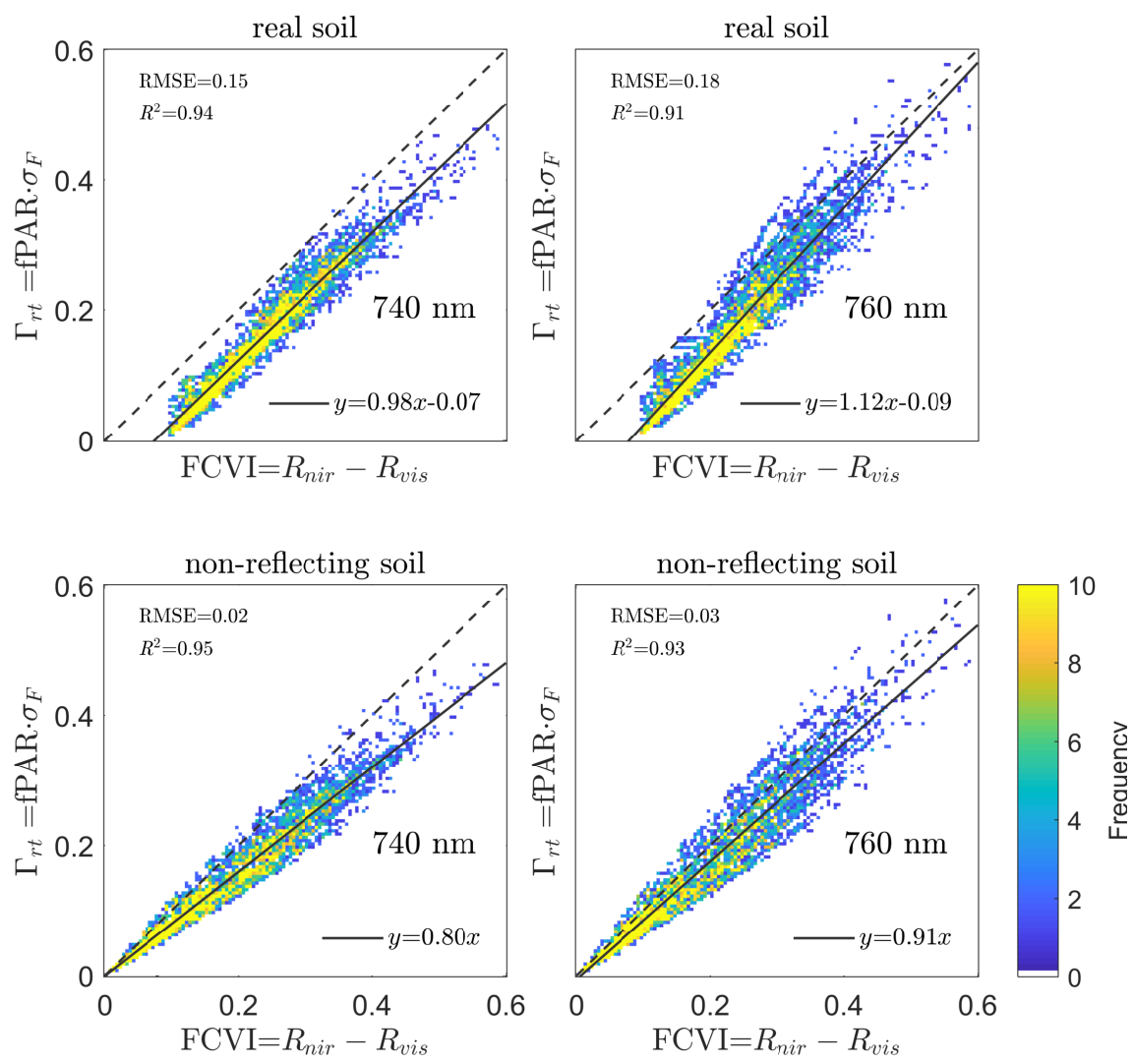

Figure 4.5: Comparison between SCOPE simulated fluorescence correcting vegetation index (FCVI) with the radiative transfer factor of fluorescence $\left(\Gamma_{r t}\right)$ at $760 \mathrm{~nm}$ and $740 \mathrm{~nm}$ for the 10800 scenarios with real soil and nonreflecting soil. The colours represent the number of scenario in a 0.005 (FCVI) by $0.005\left(\Gamma_{r t}\right)$ grid. 


\subsection{Mapping global GOME-2 SIF, NDVI, PAR and fluorescence emission efficiency}

Fig. 4.6 shows the fluorescence efficiency map $\left(\epsilon_{F}\right)$ for June 2014 as one example of the 120 calculated efficiency maps (10 years $\times 12$ months). The spatial patterns of $\epsilon_{F}$, NDVI and SIF are different from each other. SIF and NDVI are spatially correlated, but the correlations are degraded because SIF is affected by incoming PAR whereas NDVI is not. For example, SIF at the southern hemisphere is lower than SIF at the northern hemisphere in areas with similar NDVI. Areas with low NDVI normally show low SIF, but not necessarily low $\epsilon_{F}$. In fact, $\epsilon_{F}$ does not correlate with NDVI. $\epsilon_{F}$ nevertheless has some correlation with PAR. This is visible in the Amazon region, where $\epsilon_{F}$ is higher in the southern part than in the northern part, and the incident PAR is lower in the southern part.

The scatter plots (Fig. 4.7) from the data shown in Fig. 4.6 confirm that NDVI is correlated to SIF, but not to $\epsilon_{F}$. A positive correlation between NDVI and SIF is found. Low NDVI is associated with low SIF, and high NDVI is more likely associated with high SIF. On the contrary, NDVI has no clear correlation with $\epsilon_{F}$.

Fig. 4.8 summarizes the correlations between SIF, NDVI, $\epsilon_{F}$ and PAR of the 10 years of monthly measurements. It shows that SIF is significantly correlated with NDVI $(r=0.64, p<0.001)$, and has some correlations with PAR $(r=0.37, p<0.001) . \epsilon_{F}$ appears to be independent of vegetation greenness as expressed by NDVI and does not have a significant relationship with PAR $(r=-0.22, p<0.001)$. 
4. Canopy structure effects on SIF

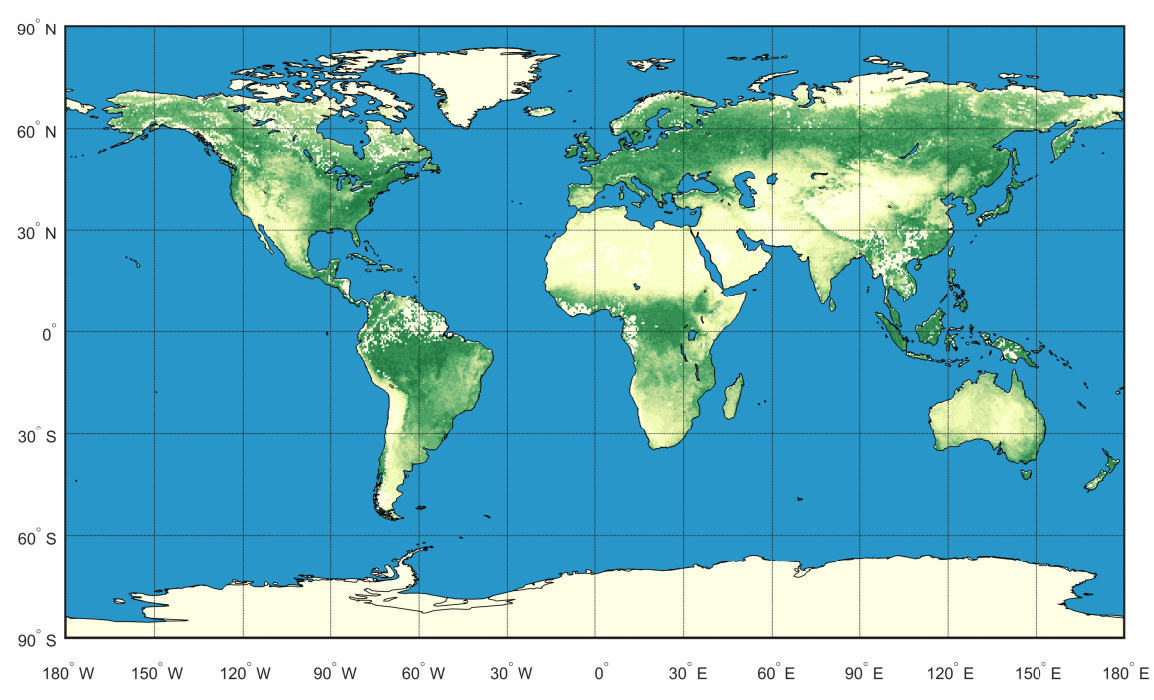

a: NDVI MODIS
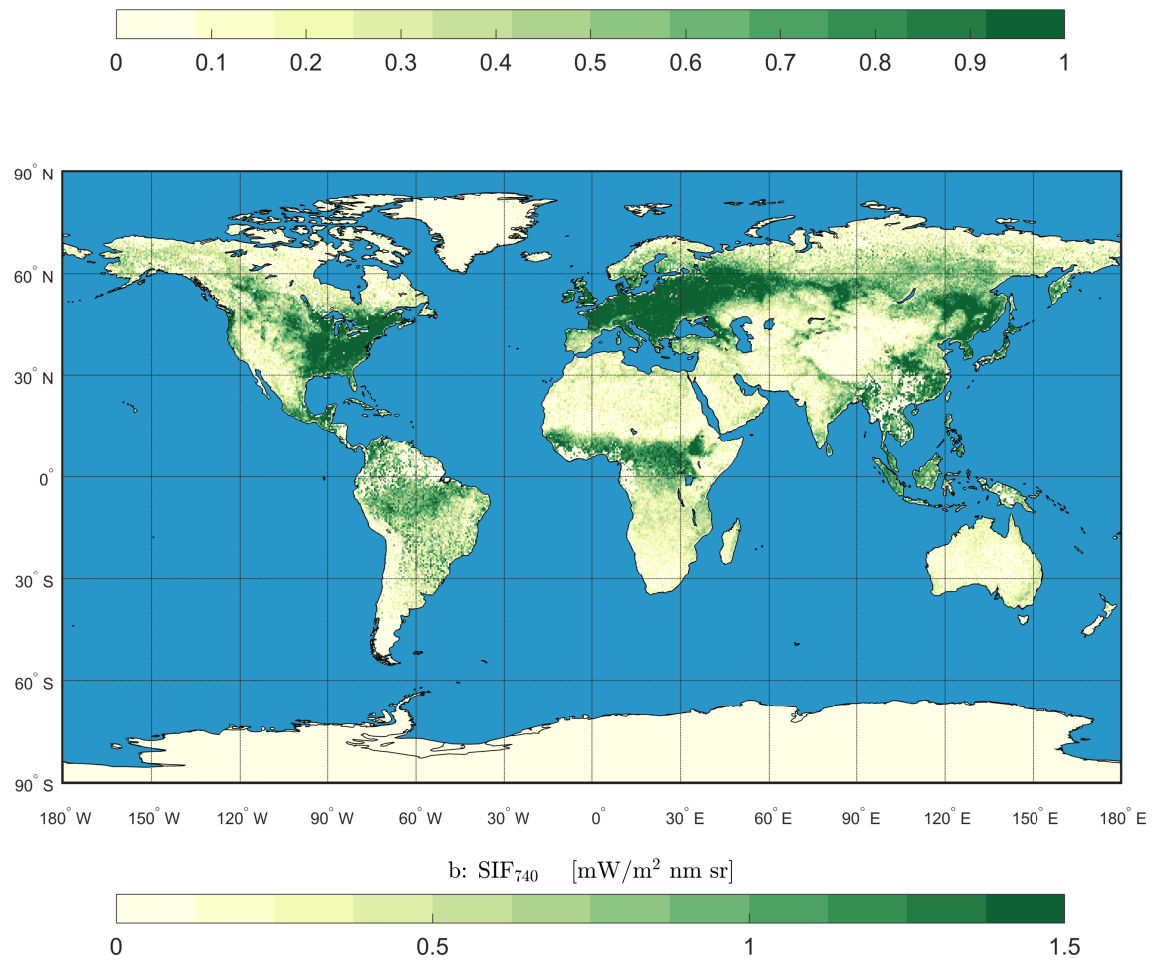


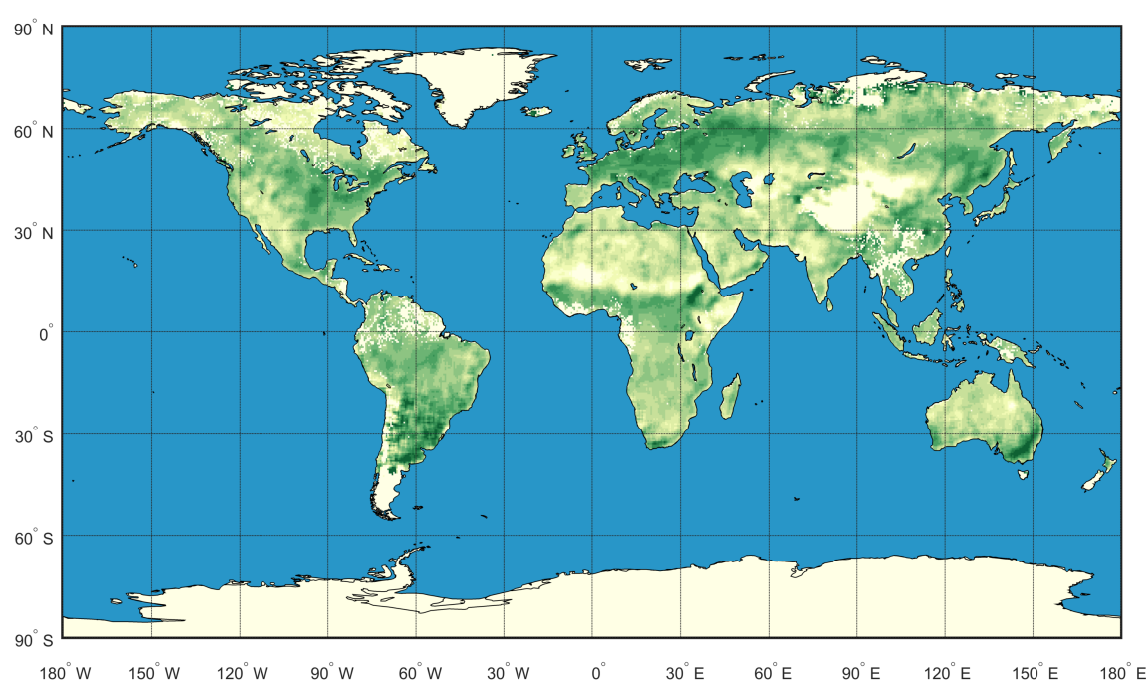

c: Fluorescence emission efficiency $\epsilon_{F} \times 10^{5}$ at $740 \mathrm{~nm}$

\begin{tabular}{|cccccc|}
\hline & 1 & 1 & 1 & 1 & \\
\hline
\end{tabular}

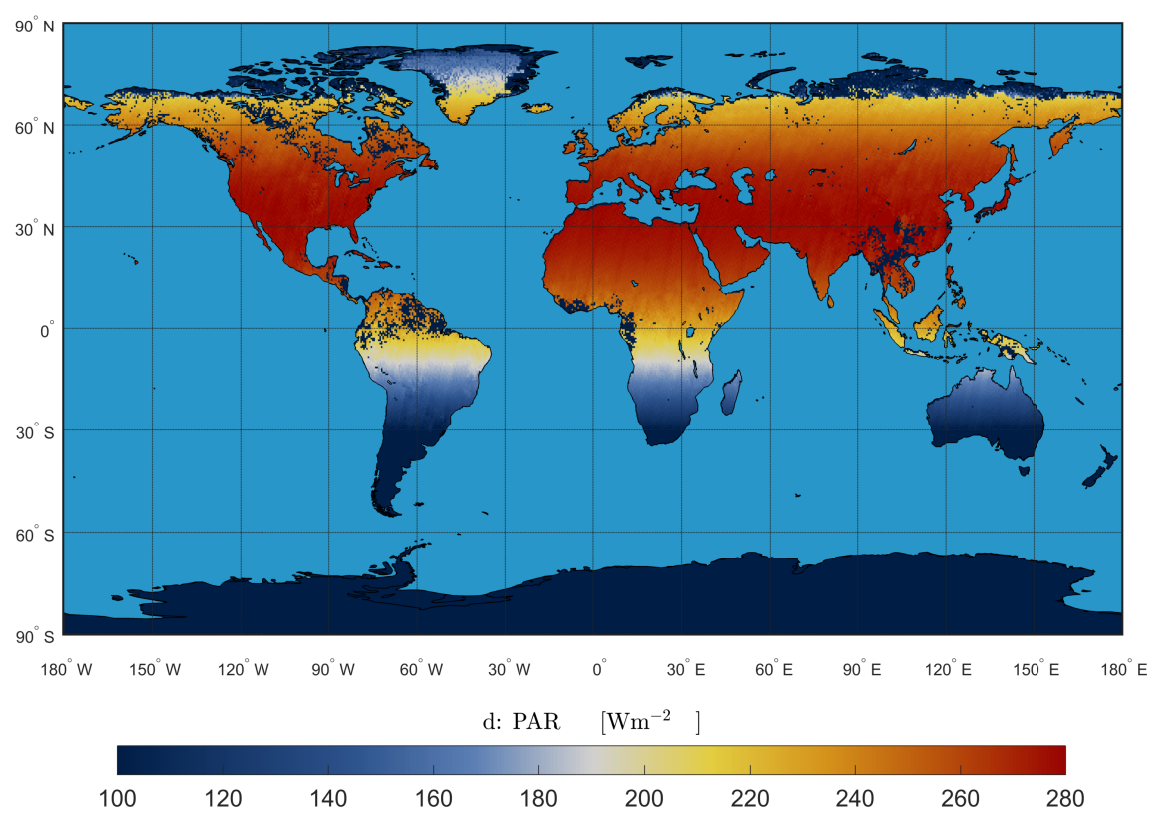

Figure 4.6: Globe maps of NDVI (a), GOME-2 SIF at $740 \mathrm{~nm}$ (b), fluorescence emission efficiency $\epsilon_{F}$ at $740 \mathrm{~nm}$ (c) and PAR (d) in June 2014. 

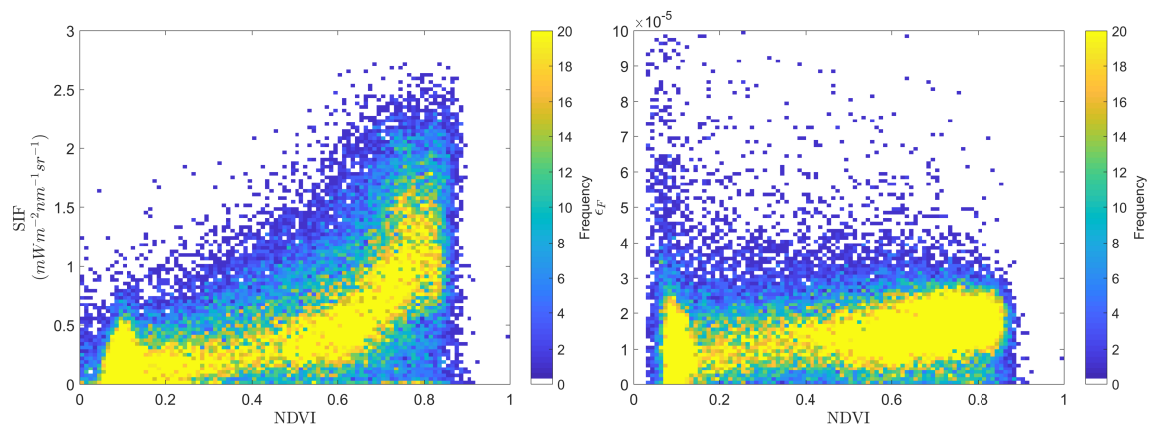

Figure 4.7: Comparison between NDVI with SIF and $\epsilon_{F}$ in June in 2014. The colours represent the number of measurements of which the $x$ and $y$ values in a small grid.

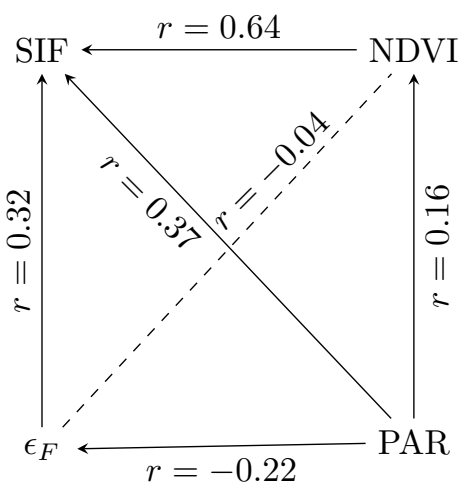

Figure 4.8: Correlations between monthly mean values gridded at 0.5 degrees by 0.5 degrees resolution of SIF, NDVI, $\epsilon_{F}$ and PAR computed for 10 years of global measurements (2007-2016). The arrows indicate the drivers (e.g. PAR is one of the drivers of SIF).

\subsection{Light response of normalized GOME-2 SIF}

Fig. 4.9 shows that the light response of the GOME-2 derived $\epsilon_{F}$ is consistent with existing studies that show that fluorescence efficiency decreases with incoming light (Genty et al., 1989; Rosema et al., 1998; Van der Tol et al., 2014). The model simulations obviously do not cover all possible cases, but the light response curves of the three cases with different $V_{c \max }$ follow a similar pattern: $\epsilon_{F}$ decreases with incoming light intensity for PAR $>50$ $\mathrm{Wm}^{-2}$. This is shown for the GOME-2-derived $\epsilon_{F}$, but not for SIF/PAR which shows increasing values for $140 \mathrm{Wm}^{-2}<\mathrm{PAR}<240 \mathrm{Wm}^{-2}$ and then 
decreasing values for $240 \mathrm{Wm}^{-2}<\mathrm{PAR}<270 \mathrm{Wm}^{-2}$. The patterns of SIF/PAR changing with light intensity is similar to that of FCVI (PAR > $140 \mathrm{Wm}^{-2}$ ). In fact, SIF/PAR and FCVI are significantly correlated with $r=0.98, p<0.001$.
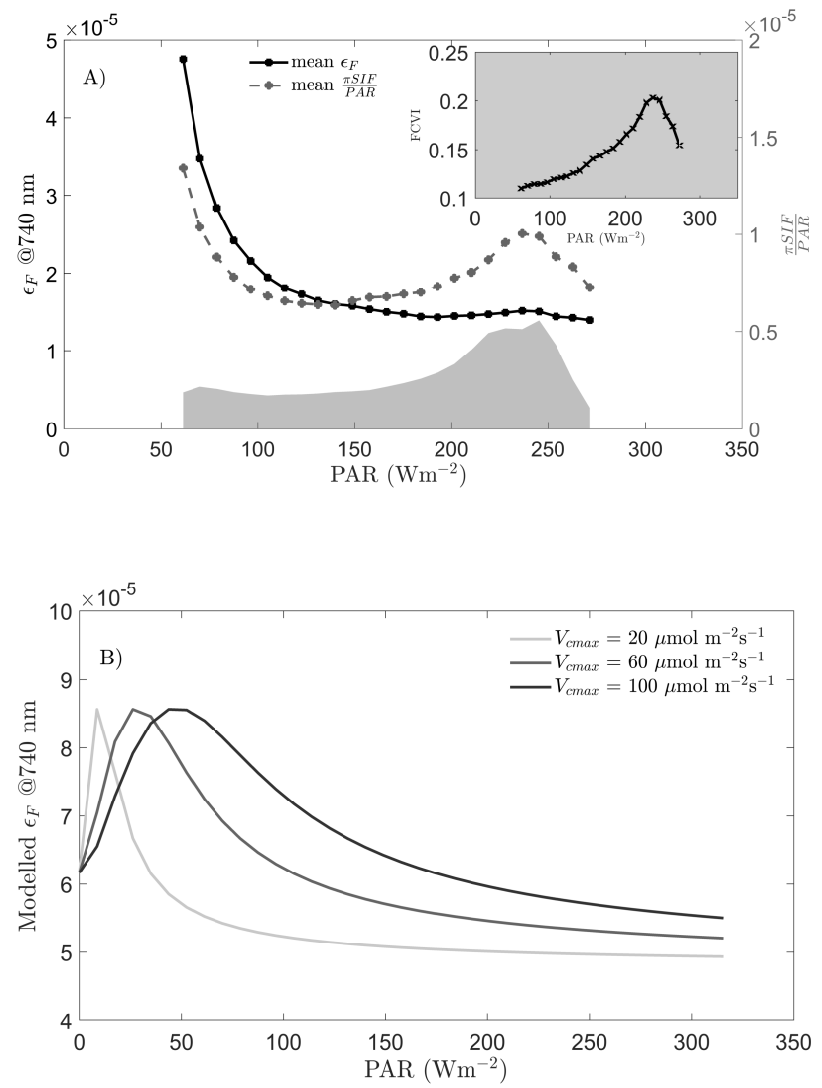

Figure 4.9: Light response of the fluorescence emission efficiency at $740 \mathrm{~nm}$ from GOME-2 SIF data (A) and from Van der Tol et al. (2014) model (B). The light responses of FCVI, $\pi$ SIF/PAR retrieved from MODIS reflectance data and GOME-2 SIF data from 2007 to 2016 are presented as well (A). FVCI is shown in the inset in the upper right corner and $\pi \mathrm{SIF} / \mathrm{PAR}$ is shown with dashed grey line. The grey area in (A) indicates the relative probability distribution function (PDF) of the pixels at various light intensities.

\section{Discussion}

The study is a follow-up and extension of Yang and van der Tol (2018), in which we analytically link canopy scattering of SIF with TOC reflectance 
and canopy interceptance. The further quantification of fPAR by using TOC reflectance and canopy interceptance results a simple index for correcting canopy structure and sun-observer geometry effects on SIF.

We use the combination of reflectance index $\left(\frac{R_{n i r}-R_{v i s}}{R_{n i r}}\right)$ and a canopy spectral invariant $\left(i_{0}\right)$ as a measure of fPAR. Our approach offers a much better estimation of fPAR than NDVI. The derivation of our index is based on a radiative transfer theory, namely the spectral invariant theory. We assume a non-reflecting background in the vegetation canopy. This allows the derivation of a simple formula for canopy absorption, scattering and TOC reflectance by using the spectral invariant theory (Smolander and Stenberg, 2005; Huang et al., 2007). Even with the assumption of non-reflecting background, the performance of our model is satisfying. Actually, not only fPAR is affected by soil background, but also TOC reflectance. The use of TOC reflectance to estimate fPAR may partially account for the soil background effects. A slight underestimation of the PPAR model is mainly due to neglecting the term after the plus sign in Eq. 4.14.

The model is a potentially useful tool for better estimation of fPAR, and this is an important line to follow. One of challenges of estimating fPAR with the spectral invariant theory is the measurement of canopy interceptance. Canopy interceptance can be estimated from the canopy LAI and leaf orientation (Stenberg and Manninen, 2015), or by inverting a RTM and retrieving reflectance data or by using LiDAR measurements (Liu et al., 2018).

On the other hand, we find that the canopy interceptance is eliminated in the product of PAR and $\sigma_{F}$, resulting in and a very simple reflectance index (FCVI). The physically-based reflectance index FCVI offers a promising way to quantify the effects of canopy structure and sun-observer geometry on far red SIF, thus separate SIF into a structural and a photochemical part. FCVI as an accurate estimator of $\Gamma_{r t}$ with $R^{2}>0.96$, yet being practically easier than any other SIF normalization so far, which provide only partial normalization of SIF. SIF/PAR (Amorós-López et al., 2008) is an estimation of fluorescence light use efficiency of a whole canopy or leaf, 
but not of photosystems. Normalizing SIF by absorbed PAR (APAR) (Moya et al., 2004) takes account the absorption of incoming light, but not the re-absorption and scattering of the emitted fluorescence. Therefore, neither SIF/PAR or SIF/APAR directly reveals light use efficiency at photosynthetic level. In contrast, FCVI quantifies the absorption of incoming light, and re-absorption and scattering of fluorescence.

FCVI only requires the commonly used vegetation VIS and NIR reflectance. Both of them are available from most optical spectral measurements as well as from many satellite missions, such as the National Aeronautics and Space Administration (NASA) Terra and Aqua satellites, the European Space Agency (ESA) Sentinel missions and the upcoming ESA FLuorescence EXplorer (FLEX) mission (Drusch et al., 2017). For instance, we used the NIR reflectance from MODIS (from Terra mission) to calculate FCVI and applied it for normalizing GOME-2 SIF data.

In applying FCVI, one should pay attention that the sun-observer geometry in the reflectance and SIF measurements are similar. The index is derived for identical geometries of reflectance and SIF, and deviations in the sun-observer geometry between reflectance and SIF could compromise the applicability. There is one-hour difference in overpass between the MODIS Terra and GOME-2 MetOp-A satellite, causing slight mismatch of sunobserver geometry in reflectance and SIF measurements and thus affecting the normalization. Better results will be obtained using instrumentation on the same satellite. Fortunately, most satellites for SIF detection measure both VIS and NIR reflectance, such that they will have identical geometry. For example, the FLEX satellite will carry the FLORIS instrument with two spectrometers: one for the Oxygen absorption bands, and another covering the full VIS and NIR spectral regions (Drusch et al., 2017). Copernicus Sentinel-3, with which FLEX will fly in tandem, will have close observation geometry to FLEX as well and offer further optical and thermal sensors.

FCVI has great potential for interpreting the remotely sensed SIF data. Normalizing SIF by FCVI and PAR results in a quantity that is much less dependent of vegetation leaf optical properties and canopy structure 
than SIF. As a result, the correlation between the 10 years of $\epsilon_{F}$ and NDVI is very low (i.e. $r=-0.04$ ). The complementarity of NDVI, $\epsilon_{F}$ and SIF can be understood as follows. NDVI is an indicator of vegetation coverage and 'greenness' (Grace et al., 2007), whereas $\epsilon_{F}$ reveals the energy distribution in the photosystems. SIF, as a more integrated signal, is a function of $\epsilon_{F}$, canopy structure, and irradiance as explained in detail by Porcar Castell et al. (2014).

$\epsilon_{F}$ reveals the photochemical response to meteorological conditions. The correlation between $\epsilon_{F}$ and illumination that we found is still low $(r=-0.22)$ due to fact that most of the available measurements are at high light condition where the relation between $\epsilon_{F}$ and PAR is relatively flat, as shown in Fig. 4.7. However, an evaluation of the shape of the light response of the normalized SIF $\left(\epsilon_{F}=\frac{\pi \text { SIF }}{\text { PAR.FCVI }}\right)$ confirms that $\epsilon_{F}$ computed is indeed the fluorescence emission efficiency of photosystems. The vegetation $\epsilon_{F}$ of the biosphere decreases with increasing PAR in the range of 50 to $250 \mathrm{Wm}^{-2}$. This response matches well with model simulations.

Interestingly, FCVI plotted versus irradiance (Fig. 4.9) peaks at a higher irradiance (about $\left.240 \mathrm{Wm}^{-2}\right)$ than $\epsilon_{F}\left(50 \mathrm{~W} \mathrm{~m}^{-2}\right)$. Note that FCVI may be considered as the result of accumulated growth and development of the vegetation, rather than the instantaneous functional condition: The difference between NIR and VIS reflectance has been regarded as an indicator of canopy greenness in the literature before (Knipling, 1970; Peñuelas and Filella, 1998). The peak at $240 \mathrm{Wm}^{-2}$ indicates that at this irradiance intensity, the areas under this illumination condition are most vegetative. In contrast, the peak of $\epsilon_{F}$ at $50 \mathrm{~W} \mathrm{~m}^{-2}$, indicates that at this irradiance, the 'pressure' on the photochemical apparatus peak due to the interplay between photochemical and non-photochemical quenching mechanisms (Van der Tol et al., 2014) .

The retrieved $\epsilon_{F}$ from GOME-2 SIF (Eq. 4.18) is lower than, but still comparable to existing knowledge as consolidated in models. The default value in SCOPE is $8.51 \times 10^{-5} \mathrm{~nm}^{-1}$ at $740 \mathrm{~nm}$, and the values retrieved range from 0 to $1 \times 10^{-4} \mathrm{~nm}^{-1}$ with an average of $2 \times 10^{-5} \mathrm{~nm}^{-1}$. There are 
several possible causes for this mismatch: 1) underestimation of SIF signal of GOME-2 SIF product, 2) overestimation of bottom-of-atmosphere PAR as a clear sky PAR was used, 3) uncertainties of reflectance products from satellite-based MODIS data, 4) the uncertainties due to assumptions in our index. The exact reasons require further investigation.

FCVI as an index for decoupling the canopy structural and plant physiological effects on SIF, opens possibilities to further study photosynthetic efficiency. Photosynthetic efficiency and fluorescence emission efficiency respond to many environmental factors in a similar way (Van der Tol et al., 2014). Retrieval of fluorescence emission efficiency thus allows for monitoring the photosynthetic functioning directly from remotely sensed data. This is relevant for determining for example the optimum growth conditions of crops and early stress detection.

\section{Conclusions}

We have proposed a physically-based model for fraction of absorbed photosynthetically active radiation (fPAR) by using the spectral invariant theory. Simulations from a radiative transfer model reveal that our model provides a better accuracy than NDVI. The model has potential to improve the estimation of fPAR from remotely sensed data.

We have also proposed a physically-based reflectance index (FCVI) to quantify the canopy structure and sun-observer geometry effects on nearinfrared SIF. The index as the difference between near-infrared and broadband visible reflectance accounts the photosynthetic light absorption, and reabsorption and scattering of fluorescence in canopies.

SIF normalized by FCVI and PAR yields the fluorescence emission efficiency. The efficiency may allows for the investigation of the energy partitioning in photosystems. The fluorescence emission efficiency directly linked to photosynthetic efficiency is expected to be an informative quantity for detecting plant stress through remote sensing. Application to GOME-2 SIF observation yields the first fluorescence emission efficiency map. The vegetation fluorescence emission efficiency response to light from GOME-2 
SIF is consistent with expectations. The index will be important as improved data sets become available from sensors such as the Sentinel 5P TROPOMI and FLEX. It is hoped that one can monitor photosynthetic functioning at regional and global scales with our approach. 


\section{Radiative transfer in multi-layer canopies*}

*This chapter is based on: Yang, P., Verhoef, W., van der Tol, C., 2017. The mSCOPE model: A simple adaptation to the SCOPE model to describe reflectance, fluorescence and photosynthesis of vertically heterogeneous canopies. Remote Sensing of Environment 201, $1-11$. 


\section{Abstract}

The vertical heterogeneity of leaf biophysical and biochemical properties may have a large effect on the bidirectional reflectance and fluorescence of vegetation canopies. This has implications for the interpretation of remote sensing data. We developed a model for light interaction and energy balance in vegetation canopies in which leaf biophysical and biochemical properties vary in the vertical. The model mSCOPE is an extension of the Soil-Canopy Observation of Photosynthesis and Energy fluxes (SCOPE) model, which simulates spectral and bidirectional reflectance, fluorescence, and photosynthesis of vertically heterogeneous vegetation canopies. The modelling of radiative transfer in mSCOPE is based on the classical SAIL theory. A solution to the radiative transfer equation for multi-layer canopies is given, which allows calculating top-of-canopy (TOC) reflectance and the flux profile. The latter is used for the simulation of fluorescence emission and photosynthesis of every leaf through the leaf radiative transfer model Fluspect and a biochemical model. The radiative transfer of fluorescence in multi-layer canopies is solved numerically in mSCOPE to obtain TOC bidirectional fluorescence. The significant effect of vertical heterogeneity of leaf properties on TOC reflectance, fluorescence and photosynthesis is demonstrated by different scenarios with customized vertical profiles of leaf chlorophyll content and leaf water content, and also with measured vertical profiles of leaf chlorophyll content in corn canopies. A preliminary validation of the reflectance calculating routine of mSCOPE is conducted by comparing measured and simulated TOC reflectance spectra of the corn canopies. We conclude that it is important to consider the vertical heterogeneity of leaf properties for the prediction of reflectance, fluorescence and photosynthesis. The model mSCOPE could serve as a tool to better understand vertically heterogeneous vegetation canopies. 


\section{Introduction}

Vegetation models are powerful tools to understand a variety of plant physiological processes. Radiative transfer models (RTMs), as a major class of vegetation models, are widely used in remote sensing applications because they offer an explicit connection between the top of canopy (TOC) observations and vegetation properties (e.g., chlorophyll, leaf area index) (Houborg et al., 2007; Ustin et al., 2009). Vegetation models that simulate photosynthesis (De Wit, 1962; Myneni, 1991) include, besides an RTM, also a leaf photosynthesis model such as Farquhar et al. (1980) or Collatz et al. (1992). The RTM simulates the light distribution within the canopy, while the photosynthesis model simulates the energy partitioning in photosystems.

SCOPE (Soil Canopy Observation, Photochemistry and Energy fluxes) is an integrated radiative transfer and energy balance model (Van der Tol et al., 2009) that simulates the spectrum of TOC reflected radiation, fluorescence emission in the viewing direction and photosynthesis as functions of leaf properties, vegetation structure, and micro-meteorological conditions. The model has been widely applied to enhance the understanding of remotely sensed data and canopy photosynthesis, and to support the quantitative use of reflectance and fluorescence for estimation of plant functional traits (Zhang et al., 2014; Damm et al., 2015a; Van der Tol et al., 2016; Drusch et al., 2017).

The SCOPE model assumes that vegetation canopies are vertically homogeneous and horizontally infinite, as its radiative transfer routines are based on the classical 1-D SAIL model (Verhoef, 1984, 1985). However, in reality, canopies generally exhibit large vertical heterogeneity of both biophysical and biochemical properties (Dreccer et al., 2000; Valentinuz and Tollenaar, 2004; Ciganda et al., 2008). Vertical heterogeneity of chlorophyll and leaf water has been found in winter wheat (Liu et al., 2015; Zhao et al., 2017), corn (Ciganda et al., 2008) and beech tree (Wang and Li, 2013). A multi-layer structure of vegetation canopies is very common, for example, forests with a grass or bush layer, field crops with a weed layer and vegetation 
in the senescent stage (Kuusk, 2001; Verhoef and Bach, 2007; Ciganda et al., 2008; Liu et al., 2015).

The vertical heterogeneity in canopies has been included in some models, and simulations with these models show that its effect on top of canopy (TOC) reflectance is not negligible (Kuusk, 2001; Verhoef and Bach, 2007; Wang and Li, 2013). However, the effect of vertical heteorgeneity on photosynthesis and fluorescence is unknown. It is expected that the vertical distribution of leaf properties can affect the light distribution in the canopy, and thereby fluorescence emission and photosynthesis of leaves. The vertical heterogeneity may also influence the re-absorption and scattering (radiative transfer) of the emitted fluorescence and thus directly affect TOC fluorescence. The simplification of vertically complex canopies to homogeneous canopies, with either mean values of phytometric and optical parameters of all leaves, or values of upper leaves, may lead to bias in the prediction of reflectance, fluorescence and photosynthesis by SCOPE. The inclusion of vertical heterogeneity of leaf properties in SCOPE will promote a better understanding of the link between remote sensing observations and plant functional traits.

This study presents a multi-layer reflectance, fluorescence and photosynthesis model based on SCOPE, called mSCOPE. The model mSCOPE considers the vertical variation of leaf biochemical and biophysical properties. The analytical solution of radiative transfer of the incident fluxes in SAIL (Verhoef, 1984) and the numerical solution of radiative transfer of the emitted fluorescence in SCOPE (Van der Tol et al., 2009) are not applicable in $\mathrm{mSCOPE}$, because the assumption of the vertical homogeneity of canopy components (leaves) does not hold in mSCOPE. Therefore, we briefly introduce the theory of mSCOPE by giving the solutions of radiative transfer of incoming radiation and emitted fluorescence in multi-layer canopies. Several example simulations are presented to illustrate the effects of vertical heterogeneity of leaf chlorophyll and leaf water content on TOC reflectance, fluorescence and canopy photosynthesis. The model mSCOPE is also preliminary validated. 


\section{Description of $\mathrm{mSCOPE}$}

\subsection{Overview}

The model mSCOPE extends the 1-D model SCOPE for a homogeneous canopy to a vertically heterogeneous vegetation canopy. It has the same architecture of SCOPE: leaf and canopy RTMs combined with an energy balance model. At leaf level, Fluspect (Vilfan et al., 2016) is used to simulate leaf reflectance, transmittance, and fluorescence. At canopy level, RTMo and RTMf (Van der Tol et al., 2016), which are two SAIL based models, compute the radiative transfer of incident radiation and emitted fluorescence, respectively. All the four models (Fluspect, RTMo, RTMf, and the energy balance model) are internally connected. Fluspect provides necessary input for canopy RMTo and RMTf. RTMo predicts the distribution of irradiance and net radiation over surface elements (leaves and soil), which are inputs to the energy balance module and RTMf.

Table 5.1: Main input parameters of SCOPE

\begin{tabular}{lllll}
\hline Parameter & Explanation & Unit & Standard value & Range \\
\hline$C_{a b}$ & Chlorophyll $a+b$ content & $\mu \mathrm{g} \mathrm{cm}^{-2}$ & 40 & $0-100$ \\
$C_{d m}$ & Leaf mass per unit area & $\mathrm{g} \mathrm{cm}^{-2}$ & 0.01 & $0-0.02$ \\
$C_{w}$ & Equivalent water thickness & $\mathrm{cm}$ & 0.015 & $0-0.05$ \\
$C_{s}$ & Senescence material (brown pigments) & fraction & 0.1 & $0-1$ \\
$C_{c a}$ & Carotenoid content & $\mu \mathrm{g} \mathrm{cm}^{-2}$ & 10 & $0-30$ \\
$N_{l}$ & Leaf structure parameter & - & 1.5 & $1-3$ \\
LAI & Leaf area index & - & 3 & $0-6$ \\
LIDFa & Leaf inclination function parameter a & - & -0.35 & $-1-1$ \\
LIDFb & Leaf inclination function parameter b & - & -0.15 & $-1-1$ \\
$\epsilon_{1}$ & fluorescence efficiency of photosystem I & - & 0.004 & $0-0.01$ \\
$\epsilon_{2}$ & fluorescence efficiency of photosystem II & - & 0.02 & $0-0.05$ \\
$\theta_{s}$ & sun zenith angle & $\circ$ & 45 & $0-90$ \\
$\psi$ & relative azimuthal angle & $\circ$ & 0 & $0-360$ \\
$\mathrm{PAR}$ & photosynthetically active radiation & $\mu \mathrm{mol} \mathrm{m}^{-2} \mathrm{~s}^{-1}$ & 1200 & $0-2200$ \\
\hline
\end{tabular}

Table 5.2: Extra input parameters of mSCOPE compared with SCOPE

\begin{tabular}{lccccc}
\hline \multicolumn{5}{c}{ mSCOPE } & SCOPE \\
\hline layer index & 1 & 2 & $\ldots$ & $\mathrm{N}$ & $v_{\text {canopy }}$ \\
leaf properties & $v(1)$ & $v(2)$ & $\ldots$ & $v(N)$ & $L_{c a n o p y}$ \\
LAI & $L(1)$ & $L(2)$ & $\ldots$ & $L(N)$ & \\
\hline \multicolumn{7}{l}{ Note: leaf properties parameters include $C_{a b}, C_{d m}, C_{w}, C_{s}, C_{c a}$ and $N_{l}}$.
\end{tabular}

The model mSCOPE retains the assumption of homogeneity in the 
horizontal direction in SCOPE, but it incorporates vertical heterogeneity of leaf properties. The type of input parameters in mSCOPE is the same as in SCOPE (Table 5.1). The difference is that mSCOPE accepts different values of leaf properties for up to 60 layers (Table 5.2). In other words, the user is allowed to deviate from the default, uniform profile of the leaf properties, and specify vertical profiles. The operational efficiency of $\mathrm{mSCOPE}$ is similar to that of SCOPE, and the same output variables are generated.

\subsection{Radiation fluxes}

In order to calculate photosynthesis and fluorescence, the radiation distribution in the canopy is required. In $\mathrm{mSCOPE}$, this is computed using the classical SAIL 4-stream theory. The radiative transfer of the direct solar flux $\left(E_{s}\right)$, downward diffuse flux $\left(E^{-}\right)$, upward diffuse flux $\left(E^{+}\right)$and flux in the viewing direction $\left(E_{o}\right)$, is analytically represented by four linear equations:

$$
\begin{aligned}
& \frac{d E_{s}}{L d x}=k E_{s} \\
& \frac{d E^{-}}{L d x}=-s E_{s}+a E^{-}-\sigma E^{+} \\
& \frac{d E^{+}}{L d x}=s^{\prime} E_{s}+\sigma E^{-}-a E^{+} \\
& \frac{d E_{o}}{L d x}=w E_{s}+v E^{-}+v^{\prime} E^{+}-K E_{o}
\end{aligned}
$$

where $x$ is the vertical relative height to the canopy bottom surface, and $L$ is canopy LAI. The extinction coefficients ( $k$ and $K$ ) depend on canopy structural characteristics (i.e., LAI and leaf angle distribution) and sunobserver geometry. The scattering coefficients $\left(s, a, \sigma, s^{\prime}, w, v, v^{\prime}\right)$ depend on canopy structural characteristics, sun-observer geometry and the optical characteristics (i.e., leaf reflectance $\rho$ and transmittance $\tau$ ) of foliar elements. These nine coefficients, first defined by Verhoef (1984), are given in Appendix A.

In mSCOPE, due to the consideration of vertical leaf properties heterogeneity, leaf reflectance, transmittance and the scattering coefficients may 
vary vertically. This has no impact on the extinction coefficients ( $K$ and $k$ ). Therefore, only the calculation of the diffuse fluxes $\left(E^{-}\right.$and $\left.E^{+}\right)$needs to be adapted in $\mathrm{mSCOPE}$, while the calculation of the directional fluxes remains the same as in SCOPE (i.e., Eq. 5.1a and 5.1d).

The vegetation layer's scattering matrix is given by

$$
\left[\begin{array}{cccc}
\tau_{s s} & 0 & 0 & 0 \\
\tau_{s d} & \tau_{d d} & \rho_{d d} & 0 \\
\rho_{s d} & \rho_{d d} & \tau_{d d} & 0 \\
\rho_{s o} & \rho_{d o} & \tau_{d o} & \tau_{o o}
\end{array}\right]=\left[\begin{array}{cc}
T_{d} & R_{b} \\
R_{t} & T_{u}
\end{array}\right]
$$

where the subscripts attached to the vectors in the left matrix refer to the direct solar $(s)$ flux, diffuse $(d)$ flux and flux in the observer's $(o)$ direction, and the subscripts attached to the right matrix denote downward $(d)$, upward $(u)$, top $(t)$ and bottom $(b) . \rho_{x 1 x 2}$ and $\tau_{x 1 x 2}$ (i.e., $x 1$ and $x 2$ are $s, d$ or $o$ ) are reflectance and transmittance of the layer for the case of flux $x 1$ to flux $x 2 . R_{t}$ and $R_{b}$ are the reflectance at top and bottom of the vegetation layer, respectively. $T_{d}$ and $T_{u}$ are the downward and upward transmittance, respectively.

The vegetation layer is normally on top of a reflecting surface (e.g., soil). The surface bidirectional reflectance $\left(R_{\text {bottom }}\right)$ is described by

$$
\left[\begin{array}{ll}
R_{s d} & R_{d d} \\
R_{s o} & R_{d o}
\end{array}\right]=R_{\text {bottom }}
$$

For a vertically homogeneous canopy (as in SCOPE), the analytical solution to the canopy scattering matrix (Eq. 5.2) is first solved, then the TOC reflectance and flux profile are computed (Verhoef, 1984, 1985). However, for a vertically heterogeneous canopy (as in $\mathrm{mSCOPE}$ ), it is difficult to get an analytical solution to the canopy scattering matrix: For each vegetation layer, the scattering matrix is different due to heterogeneous leaf properties. Therefore, in mSCOPE, we avoid the calculation of canopy scattering matrix. Instead, we developed a new strategy. The main idea comes from the adding method originally developed to simulate TOC reflectance in heterogeneous canopies (Cooper et al., 1982; Verhoef, 1985). In mSCOPE, we extended it to the calculation of the flux profile and TOC fluorescence. 
The procedure is summarized as follows: 1 . divide the vertical heterogeneous layer into several homogeneous layers; 2 . start from the bottom homogeneous layer, calculate the surface reflectance of the combined system of the bottom surface (e.g., soil) and this layer; 3. add a new homogeneous vegetation layer above the surface of the previous system in step 2, and calculate the surface reflectance of the new system; 4 . repeat step 3 until all homogeneous layers are added. 5. once the surface reflectance at each vertical level is obtained, the fluxes profile can be computed from top to bottom, given the incident flux at top of the canopy.

In mSCOPE, the properties of a user defined N-layer canopy (where $\mathrm{N}<=60$ ) are distributed over 60 sublayers. The use of 60 sublayers is similar to SCOPE, and it is necessary for computational reasons: Sufficiently thin sublayers of no more than 0.1 units of leaf area index (LAI) are needed to avoid problems in the numerical discretization of the differential equations. We use the term 'layers' for a layered canopy (such as understory and overstory), and the term 'sublayers' for the numerical discretization of the canopy.

In a heterogeneous 60 -sublayer system that is bounded by a surface at the bottom, we distinguish the 60 sublayers by numbers from 1 to 60 , and the fluxes at the bottom and the top of the system by the numbers 1 and 61 . The levels at the interfaces between neighbouring sublayers are numbered from 2 to 60 . Using this numbering for sublayers and their interfaces, the following set of equations describe radiative transfer in the whole system:

$$
\begin{aligned}
& E^{u}(1)=R_{\text {bottom }} E^{d}(1) \\
& {\left[\begin{array}{l}
E^{d}(1) \\
E^{u}(2)
\end{array}\right]=\left[\begin{array}{ll}
t_{d}(1) & r_{b}(1) \\
r_{t}(1) & t_{u}(1)
\end{array}\right]\left[\begin{array}{l}
E^{d}(2) \\
E^{u}(1)
\end{array}\right]} \\
& \vdots \\
& {\left[\begin{array}{l}
E^{d}(60) \\
E^{u}(61)
\end{array}\right]=\left[\begin{array}{ll}
t_{d}(60) & r_{b}(60) \\
r_{t}(60) & t_{u}(60)
\end{array}\right]\left[\begin{array}{l}
E^{d}(61) \\
E^{u}(60)
\end{array}\right]}
\end{aligned}
$$


where

$$
E^{d}=\left[\begin{array}{c}
E_{s} \\
E^{-}
\end{array}\right] ; E^{u}=\left[\begin{array}{c}
E^{+} \\
E_{o}
\end{array}\right]
$$

Except for the bottom reflectance $R_{\text {bottom }}$, all reflectance and transmittance matrices here refer to all the sublayers. These are therefore represented by lower case letters (i.e., $t$ and $r$ ).

If only the downward fluxes at the top of the system are given, by the elements of the vector $E^{d}(61)$, all other flux vectors can be derived. To this end, we first solve the combination of the bottom with sublayer 1 , which is given by the equations (i.e., from Eq. 5.4a and 5.4b)

$$
\begin{aligned}
E^{u}(1) & =R_{\text {bottom }} E^{d}(1) \\
E^{d}(1) & =t_{d}(1) E^{d}(2)+r_{b}(1) E^{u}(1) \\
& =t_{d}(1) E^{d}(2)+r_{b}(1) R_{\text {bottom }} E^{d}(1) \\
& =\left[I-r_{b}(1) R_{\text {bottom }}\right]^{-1} t_{d}(1) E^{d}(2) \\
E^{u}(2) & =r_{t}(1) E^{d}(2)+t_{u}(1) E^{u}(1) \\
& =r_{t}(1) E^{d}(2)+t_{u}(1) R_{\text {bottom }} E^{d}(1)
\end{aligned}
$$

Here we introduced a new quantity $X=\left(I-r_{b} R_{b o t t o m}\right)^{-1} t_{d}$, which is called the effective downward transmittance, since it describes the relationship between the downward fluxes at successive levels while taking into account the multiple reflections with the thick layer under the level of interest. Eq. ?? and Eq. 5.8 are expressed as:

$$
\begin{aligned}
& E^{d}(1)=X(1) E^{d}(2) \\
& E^{u}(2)=\left[r_{t}(1)+t_{u}(1) R_{\text {bottom }} X(1)\right] E^{d}(2)
\end{aligned}
$$


Eq. 5.10 gives the calculation of the upward flux at the top of sublayer 1. From Eq. 5.10, we obtain a new effective surface reflectance matrix at the top of sublayer 1 , given by

$$
R(2)=r_{t}(1)+t_{u}(1) R_{\text {bottom }} X(1)
$$

In the following, we will use capital letters to indicate quantities that refer to all levels from the bottom (level 1) to the level of interest, and lower case letters to indicate quantities that only refer to a single thin sublayer. Thus, we may also identify $R_{\text {bottom }}$ as $R(1)$, which is usually given as an input (e.g., soil reflectance).

In this way we obtain a recursive rule that can be extended up until the top of the whole system of sublayers. We use the equations

$$
\begin{aligned}
& X(j)=\left[I-r_{b}(j) R(j)\right]^{-1} t_{d}(j) \\
& R(j+1)=r_{t}(j)+t_{u}(j) R(j) X(j)
\end{aligned}
$$

The matrices $X(j)$ are derived in Appendix B and written as

$$
X(j)=\left[\begin{array}{cc}
\tau_{s s}(j) & 0 \\
\frac{\tau_{s d}(j)+\tau_{s s}(j) R_{s d}(j) \rho_{d d}(j)}{1-\rho_{d d}(j) R_{d d}(j)} & \frac{\tau_{d d}(j)}{1-\rho_{d d}(j) R_{d d}(j)}
\end{array}\right]=\left[\begin{array}{cc}
X_{s s}(j) & 0 \\
X_{s d}(j) & X_{d d}(j)
\end{array}\right]
$$

The part $1 /\left(1-\rho_{d d} R_{d d}\right)$ includes the repeated reflections of radiation between a surface and the bottom of a vegetation layer in the radiative transfer. By going from bottom to top, the final result obtained is the surface reflectance matrix at the top of all sublayers, $R(61)$.

After completion of the first loop, and since each $X$ matrix connects the downward fluxes at level $j$ to those at the next higher level $j+1$, we may start at the top level, for which the downward incident fluxes are given, and then derive the downward fluxes at all successive deeper levels by employing the previously stored $X$ matrices. At the same time, one can derive the upward fluxes by using the stored $R$ matrices. The equations used here are

$$
E^{d}(j)=X(j) E^{d}(j+1)
$$




$$
E^{u}(j)=R(j) E^{d}(j)
$$

With all scattering and extinction coefficients in Eq. 5.1 defined per unit of LAI, we can write for each sublayer with a small LAI ( $\Delta L$, $1 / 60$ of total canopy LAI). We can establish the scattering and extinction coefficients for all sublayers, and convert them into thin layer reflectances and transmittances:

$$
\begin{aligned}
& \tau_{s s}(j)=1-k(j) \Delta L \\
& \tau_{d d}(j)=1-a(j) \Delta L \\
& \tau_{s d}(j)=s^{\prime}(j) \Delta L \\
& \rho_{s d}(j)=s(j) \Delta L \\
& \rho_{d d}(j)=\sigma(j) \Delta L
\end{aligned}
$$

A complete algorithm to calculate the flux profile in a whole canopy layer of vertically heterogeneous vegetation is summarized as:

For sublayer $j=1$ to 60 (bottom to top)

$$
\begin{aligned}
& X_{s s}(j)=\tau_{s s}(j) \\
& X_{s d}(j)=\frac{\tau_{s d}(j)+\tau_{s s}(j) R_{s d}(j) \rho_{d d}(j)}{1-\rho_{d d}(j) R_{d d}(j)} \\
& X_{d d}(j)=\frac{\tau_{d d}(j)}{1-\rho_{d d}(j) R_{d d}(j)} \\
& R_{s d}(j+1)=\rho_{s d}(j)+\tau_{d d}(j)\left[R_{s d}(j) X_{s s}(j)+R_{d d}(j) X_{s d}(j)\right] \\
& R_{d d}(j+1)=\rho_{d d}(j)+\tau_{d d}(j) R_{d d}(j) X_{d d}(j)
\end{aligned}
$$

For sublayer $j=60$ to 1 (top to bottom)

$$
\begin{aligned}
& E_{s}(j)=X_{s s}(j) E_{s}(j+1) \\
& E^{-}(j)=X_{s d}(j) E_{s}(j+1)+X_{d d}(j) E^{-}(j+1) \\
& E^{+}(j)=R_{s d}(j) E_{s}(j)+R_{d d}(j) E^{-}(j)
\end{aligned}
$$


$E_{s}(61)$ and $E^{-}(61)$ refer to incident direct solar flux and diffuse flux, which are $E_{\text {sun }}$ and $E_{\text {sky }}$. The hemispherical fluxes $E^{-}, E^{+}$profiles and hemispherical reflectance factors $R_{d d}$ and $R_{s d}$ are computed.

\subsection{The observed radiance}

The adding method can be used to calculate the flux in the observer's direction $E_{o}$ and the directional reflectance factors $R_{s o}$ and $R_{d o}$. However, the hot spot effects on $R_{s o}$ and $E_{o}$ are not considered in this method. Thus, $R_{s o}$ and $E_{o}$ is given separately by solving Eq. $5.1 \mathrm{~d}$.

In a leaf canopy with finite leaf size the solar flux is described statistically, using the probability of sunshine and its complement, the probability of being in the shade. The probability of sunshine is described with a Poisson model as:

$$
P_{s}(j)=e^{k \Delta L(j-60)}
$$

The gap probability $P_{s}$ controls the probability of sunshine. For example, at sunlit locations the solar flux equals to the solar flux incident at the top of the canopy, $E_{s}(61)$ or $E_{\text {sun }}$ (i.e., incident direct solar flux). The probability for leaves in sublayer $j$ (i.e., $j$ from 1 to 60 ) or the soil (i.e., $j=0$ ) of being observed through direct line-of-sight by an observer above the canopy is expressed by a similar function:

$$
P_{o}(j)=e^{K \Delta L(j-60)}
$$

The observed radiance contributed by the leaves is obtained by numerically solving Eq. 5.1d:

$$
\begin{aligned}
& E_{o}^{\text {leaves }}(61)=\Delta L \sum_{j=1}^{60}\left[w(j) E_{s}(j)+v(j) E^{-}(j)+v^{\prime}(j) E^{+}(j)\right] P_{o}(j) \\
& =\Delta L \sum_{j=1}^{60}\left[w(j) E_{\text {sun }} P_{s}(j)+v(j) E^{-}(j)+v^{\prime}(j) E^{+}(j)\right] P_{o}(j) \\
& =\Delta L \sum_{j=1}^{60}\left\{w(j) E_{\text {sun }} P_{s}(j) P_{o}(j)+\left[v(j) E^{-}(j)+v^{\prime}(j) E^{+}(j)\right] P_{o}(j)\right\}
\end{aligned}
$$


In order to take proper account of the hot spot effects, the product of the correlated probabilities $P_{s} P_{o}$, which indicates the joint probability of directly observing, through gaps in the canopy, sunlit phyto-elements or sunlit soil, must be replaced by the so-called bi-directional gap probability $P_{s o}$ (Verhoef, 1998; Van der Tol et al., 2009), which is given in Appendix C. Therefore we write

$$
E_{o}^{\text {leaves }}(61)=\Delta L \sum_{j=1}^{60}\left\{w(j) E_{\text {sun }} P_{\text {so }}(j)+\left[v(j) E^{-}(j)+v^{\prime}(j) E^{+}(j)\right] P_{o}(j)\right\}
$$

The contribution from the soil is given by.

$$
E_{o}^{\text {soil }}(61)=E_{\text {sun }} P_{\text {so }}(0) R_{\text {so }}(1)+R_{d o}(1) E^{-}(1) P_{o}(0)
$$

where $R_{s o}(1)$ and $R_{d o}(1)$ are the directional reflectance factors of the background $\left(R_{\text {bottom }}\right)$.

For the total TOC radiance (times $\pi$ ), we find in the general case

$$
\pi L_{o}=E_{o}^{\text {soil }}(61)+E_{o}^{\text {leaves }}(61)
$$

The reflectance of canopy observed by a sensor is given by

$$
\rho=\frac{\pi L_{o}}{E_{\text {sun }}+E_{\text {sky }}}
$$

where $E_{\text {sun }}$ and $E_{s k y}$ are given as input or simulated from atmosphere radiative transfer models, such as MODTRAN (Berk et al., 2005).

The directional reflectance factors $R_{s o}$ and $R_{d o}$ are computed by using Eq. 5.26 setting $E_{s k y}$ or $E_{\text {sun }}$ to 0 (i.e., when $E_{s k y}=0, \rho=R_{s o}$ ).

\subsection{Photosynthesis}

Once the fluxes profile is computed, the radiation absorbed by the foliage can be calculated. The radiation absorbed by chlorophyll is used for photosynthesis, fluorescence and heat dissipation (Baker, 2008). Photosynthesis is then calculated as a product of the absorbed radiation and photosynthetic efficiency. The efficiencies of photosynthesis and fluorescence 
emission are simulated by a biochemical model as functions of the absorbed light, leaf temperature and $\mathrm{CO}_{2}$ concentration and other factors (Van der Tol et al., 2014).

To calculate the total photosynthesis, the canopy has to be divided into multiple (e.g., 60 in mSCOPE) thin sublayers, as described in section 2.2 . Leaves in a thin sublayer are assumed to have the same ambient conditions including temperature and humidity, while the incident light on individual leaf in the thin layer may be different. Sunlit and shaded leaves are considered separately, in terms of the efficiencies of photosynthesis and fluorescence. For shaded leaves which are only illuminated by diffuse light, their efficiencies depend on their vertical relative height $(j)$, expressed as $\epsilon_{F}(j)$ and $\epsilon_{P}(j)$. For sunlit leaves which are illuminated by both direct solar light and diffuse light, their efficiencies depend on their orientation (leaf zenith angle $\theta_{l}$, azimuth angle $\left.\varphi_{l}\right)$ and vertical relative height $(j)$, expressed as $\epsilon_{F}\left(j, \theta_{l}, \varphi_{l}\right)$ and $\epsilon_{P}\left(j, \theta_{l}, \varphi_{l}\right)$. Fluorescence and photosynthesis of the sunlit leaves are calculated for each specific leaf orientation. Numerically, 13 discrete leaf inclinations $\theta_{l}$ are used as in mSCOPE inherited from SCOPE and SAIL, and the uniform leaf azimuth $\varphi_{l}$ distribution is also discretized to 36 angles of 5 , $15, \ldots, 355^{\circ}$ relative to solar azimuth. At each sublayer, the leaf inclination distribution is described by using mathematical functions (LIDF) (De Wit, 1965; Verhoef and Bunnik, 1975) which quantify the probability of each leaf orientation class.

Canopy net photosynthesis $\left(A, \mu \mathrm{mol} \mathrm{CO}_{2} \mathrm{~m}^{-2} \mathrm{~s}^{-1}\right)$ is then expressed as:

$$
A=\Delta L \sum_{j=1}^{60}\left\{\left[1-P_{s}(j)\right] \cdot A_{h}(j)+\sum_{36 \varphi_{l}, 13 \theta_{l}} P_{s}(j) \cdot P\left(\varphi_{l}, \theta_{l}\right) \cdot A_{s}\left(j, \varphi_{l}, \theta_{l}\right)\right\}
$$

where $P_{s}$ is the probability of sunlit leaves and $\left(1-P_{s}\right)$ is the probability of shaded leaves in sublayer $j . A_{h}(j)$ is the photosynthesis of shaded leaves in sublayer $j$ per unit leaf area. $A_{s}\left(j, \varphi_{l}, \theta_{l}\right)$ is photosynthesis of sunlit leaves in sublayer $j$ with the leaf orientation of $\left(\varphi_{l}, \theta_{l}\right)$ per unit leaf area. $P\left(\varphi_{l}, \theta_{l}\right)$ is the probability of leaves with given leaf orientation $\left(\varphi_{l}, \theta_{l}\right)$, which has $13 \times 36$ classes in the model. $P\left(\varphi_{l}, \theta_{l}\right)$ given by LIDF is identical for each sublayer 
in the canopy, because LIDF does not vary vertically in mSCOPE.

\subsection{Fluorescence}

Similar to SCOPE, the emission and radiative transfer of fluorescence are both modelled in mSCOPE. However, the vertical heterogeneity of leaf properties, have made the previous equations not applicable in this scenario. To account for this, new equations and solutions have been implemented in mSCOPE. To model the radiative transfer of fluorescence, we use a similar strategy as the adding method used in modelling of fluxes profile, described in section 2.2 .

Fluorescence emitted by the foliage only consists of diffuse fluxes. Only radiative transfer of the upward and downward diffuse fluxes should be described. This can be established similarly to Eq. 5.4 and shown as follows.

$$
\begin{aligned}
& E_{F}^{-}(j)=\tau_{d d}(j) E_{F}^{-}(j+1)+\rho_{d d}(j) E_{F}^{+}(j)+F_{\text {em }}^{-}(j) \\
& E_{F}^{+}(j)=\rho_{d d}(j) E_{F}^{-}(j+1)+\tau_{d d}(j) E_{F}^{+}(j)+F_{\text {em }}^{+}(j)
\end{aligned}
$$

where $F_{e m}^{-}$and $F_{e m}^{+}$are the downward ('+') and upward ('-') diffuse hemispherical emitted fluorescence of a sublayer. They are excited by the direct solar flux $\left(E_{s}\right)$, upward $\left(E^{+}\right)$and downward $\left(E^{-}\right)$diffuse light at each spectral band $\left(\lambda_{e}\right)$ from 400 to $700 \mathrm{~nm}$.

$$
\begin{aligned}
& F_{e m}^{-}(j)=\Delta L \int_{400}^{700}\left[s_{f}^{\prime}(j) E_{s}(j+1)+\sigma_{f}^{\prime}(j) E^{-}(j+1)+\sigma_{f}(j) E^{+}(j)\right] d \lambda_{e} \\
& F_{e m}^{+}(j)=\Delta L \int_{400}^{700}\left[s_{f}(j) E_{s}(j+1)+\sigma_{f}(j) E^{-}(j+1)+\sigma_{f}^{\prime}(j) E^{+}(j)\right] d \lambda_{e}
\end{aligned}
$$

where $\Delta L$ is LAI of a thin sublayer which is $1 / 60$ of canopy LAI. The emission coefficients (with subscript $f$ ) are determined by sun-observer geometry, canopy structure, leaf optical properties and fluorescence emission efficiency of photosystems and given in Appendix A. The emission efficiencies in Eq. 5.29 are effective values for a sublayer, in which fluorescence emission is 
considered separately for sunlit and shaded leaves due to their different fluorescence emission efficiencies.

The flux at the top of an ensemble of sublayers may contain reflected downward flux as well as upward emitted flux, called $U$. Therefore we write

$$
E_{F}^{+}(j)=R_{d d}(j) E_{F}^{-}(j)+U(j)
$$

There is no upward emitted flux from the soil, thus $U(1)=0$. For the reflection of fluorescence by the soil we write

$$
E_{F}^{+}(1)=R_{d d}(1) E_{F}^{-}(1)
$$

Substituting Eq. 5.30 into Eq. 5.28a gives

$$
E_{F}^{-}(j)=\frac{\tau_{d d}(j) E_{F}^{-}(j+1)+\rho_{d d}(j) U(j)+F_{e m}^{-}(j)}{1-\rho_{d d}(j) R_{d d}(j)}
$$

This can be written as

$$
E_{F}^{-}(j)=X_{d d}(j) E_{F}^{-}(j+1)+Y(j)
$$

where

$$
Y(j)=\frac{\rho_{d d}(j) U(j)+F_{e m}^{-}(j)}{1-\rho_{d d}(j) R_{d d}(j)}
$$

Substituting Eq. 5.30 into Eq. 5.28b we obtain

$$
E_{F}^{+}(j+1)=\rho_{d d}(j) E_{F}^{-}(j+1)+\tau_{d d}(j)\left[R_{d d}(j) E_{F}^{-}(j)+U(j)\right]+F_{e m}^{+}(j)
$$

Substituting Eq. 5.33 into Eq. 5.35 we obtain

$$
\begin{aligned}
E_{F}^{+}(j+1) & =\rho_{d d}(j) E_{F}^{-}(j+1)+\tau_{d d}(j)\left\{R_{d d}(j)\left[X_{d d}(j) E_{F}^{-}(j+1)+Y(j)\right]+U(j)\right\}+F_{e m}^{+}(j) \\
& =\left[\rho_{d d}(j)+\tau_{d d}(j) R_{d d}(j) X_{d d}(j)\right] E_{F}^{-}(j+1)+\tau_{d d}(j)\left[R_{d d}(j) Y(j)+U(j)\right]+F_{e m}^{+}(j) \\
& =R_{d d}(j+1) E_{F}^{-}(j+1)+\tau_{d d}(j)\left[R_{d d}(j) Y(j)+U(j)\right]+F_{e m}^{+}(j)
\end{aligned}
$$

Comparing Eq. 5.36 with Eq. 5.30, we obtain

$$
U(j+1)=\tau_{d d}(j)\left[R_{d d}(j) Y(j)+U(j)\right]+F_{e m}^{+}(j)
$$

A complete algorithm to calculate the fluorescence profile in a vertically heterogeneous canopy is summarized as: 
For sublayer $j=1$ to 60 (bottom to top)

$$
\begin{aligned}
& X_{d d}(j)=\frac{\tau_{d d}(j)}{1-\rho_{d d}(j) R_{d d}(j)} \\
& Y(j)=\frac{\rho_{d d}(j) U(j)+F_{e m}^{-}(j)}{1-\rho_{d d}(j) R_{d d}(j)} \\
& R_{d d}(j+1)=\rho_{d d}(j)+\tau_{d d}(j) R_{d d}(j) X_{d d}(j) \\
& U(j+1)=\tau_{d d}(j)\left[R_{d d}(j) Y(j)+U(j)\right]+F_{e m}^{+}(j)
\end{aligned}
$$

After application of these equations from the bottom to the top of the canopy, the following two equations can be applied to calculate the hemispherical fluorescence fluxes from top to bottom:

For sublayer $j=60$ to 1 (top to bottom)

$$
\begin{aligned}
& E_{F}^{-}(j)=X_{d d}(j) E_{F}^{-}(j+1)+Y(j) \\
& E_{F}^{+}(j)=R_{d d}(j) E_{F}^{-}(j)+U(j)
\end{aligned}
$$

Where $E_{F}^{-}(61)=0, U(1)=0$ and $R_{d d}(1)$ is given in the soil reflectance matrix.

\subsection{The observed fluorescence}

The fluorescence observed at top of canopy has four contributions: 1. fluorescence emitted by sunlit leaves and directly observed via $P_{s o}(j)$, 2. fluorescence emitted by shaded leaves and directly observed via $P_{o}(j), 3$. diffuse fluorescence flux scattered in the canopy and observed via $P_{o}(j), 4$. diffuse fluorescence flux reflected by the soil and observed via $P_{o}(0)$.

Similar to Eq. 5.29, the emitted fluorescence for each sublayer is given as:

$$
F_{e m}^{o}(j)=\Delta L \int_{400}^{700}\left[w_{f}(j) E_{s}(j)+v_{f}(j) E^{-}(j)+v_{f}^{\prime}(j) E^{+}(j)\right] d \lambda_{e}
$$

The emitted fluorescence of sunlit leaves per unit layer $F_{e m}^{s o}(j)$ is distinguished from that of shaded leaves by $F_{e m}^{h o}(j)$. The four contributions 
are given by

$$
\begin{aligned}
& \pi L_{F}^{1}=\sum_{j=1}^{60} F_{e m}^{s o}(j) P_{s o}(j) \\
& \pi L_{F}^{2}=\sum_{j=1}^{60} F_{e m}^{h o}(j)\left[P_{o}(j)-P_{s o}(j)\right] \\
& \pi L_{F}^{3}=\sum_{j=1}^{60}\left[v(j) E_{F}^{-}(j)+v^{\prime}(j) E_{F}^{+}(j)\right] P_{o}(j) \\
& \pi L_{F}^{4}=E_{F}^{-}(1) R_{d o}(1) P_{o}(0)
\end{aligned}
$$

The total observed fluorescence $\left(L_{F}\right)$ is summed by:

$$
L_{F}=L_{F}^{1}+L_{F}^{2}+L_{F}^{3}+L_{F}^{4}
$$

\section{Materials and Methods}

\subsection{Synthetic dataset of two-layer canopies}

First, synthetic datasets was used to evaluate the vertical heterogeneity effect on TOC fluorescence, TOC reflectance and canopy photosynthesis. In the current experiment, scenarios for two-layer canopies have been simulated. A two-layer canopy is one of the simplest multi-layer canopies and it has also been used in several heterogeneous reflectance models, including the

\begin{tabular}{|c|c|c|c|c|}
\hline \multirow[t]{2}{*}{ Scenario } & \multicolumn{2}{|c|}{ upper layer } & \multicolumn{2}{|c|}{ lower layer } \\
\hline & $C_{a b}$ & $C_{w}$ & $C_{a b}$ & $C_{w}$ \\
\hline S0 & 40 & 0.015 & 40 & 0.015 \\
\hline $\mathrm{S} 1$ & 60 & 0.02 & 20 & 0.01 \\
\hline $\mathrm{S} 2$ & 20 & 0.01 & 60 & 0.02 \\
\hline $\mathrm{S} 3$ & 40 & 0.015 & 0 & 0.01 \\
\hline $\mathrm{S} 4$ & 40 & 0.015 & 20 & 0.02 \\
\hline $\mathrm{S} 5$ & 40 & 0.015 & 60 & 0.03 \\
\hline
\end{tabular}
two-layer canopy reflectance model (Kuusk, 2001) and 4SAIL2 (Verhoef and Bach, 2007).

Table 5.3: Input parameters of vertical leaf chlorophyll $\left(C_{a b}, \mu \mathrm{g} \mathrm{cm}{ }^{-2}\right)$ and equivalent water thickness $\left(C_{w}, \mathrm{~cm}\right)$ profile in six two-layer canopy scenarios.

Specifically, six scenarios were generated from different combinations of leaf chlorophyll $C_{a b}$ and leaf water content $C_{w}$ (Table 5.3). These six 
scenarios can be considered as two groups of canopies. In the first group, the three scenarios (S0, S1, S2) had the same canopy averaged $C_{a b}$ and $C_{w}$, which were $40 \mu \mathrm{g} \mathrm{cm}^{-2}$ and $0.015 \mathrm{~cm}$, respectively, while the vertical distributions of $C_{a b}$ and $C_{w}$ were different. In the second group, the four scenarios (S0, $\mathrm{S} 3, \mathrm{~S} 4, \mathrm{~S} 5)$ had the same $C_{a b}$ and $C_{w}$ of leaves in the upper layer, while $C_{a b}$ and $C_{w}$ varied in the lower layer. In all the scenarios, LAI of both two layers was 1.5 (total canopy LAI was 3). The other model parameters were set to the 'standard' values (Table 5.1). It should be noted that S0 was a scenario of homogeneous canopy that served as a reference.

\subsection{Field measurement dataset of corn canopy}

To further investigate the effects of vertical heterogeneity, reflectance, fluorescence and photosynthesis were simulated with field measured data as input. The field experimental data was acquired from a corn canopy, where synchronous seasonal measurements of vertical profiles of leaf chlorophyll content and LAI, TOC reflectance from 400 to $900 \mathrm{~nm}$ were available (Gitelson et al., 2003, 2006; Ciganda et al., 2008). The measurements on 3 days in the early, middle and end of the growing season were selected, which were on DOY (day of year) 173, 206 and 259 in 2005, as shown in Fig. 5.1 and Fig. 5.2 .

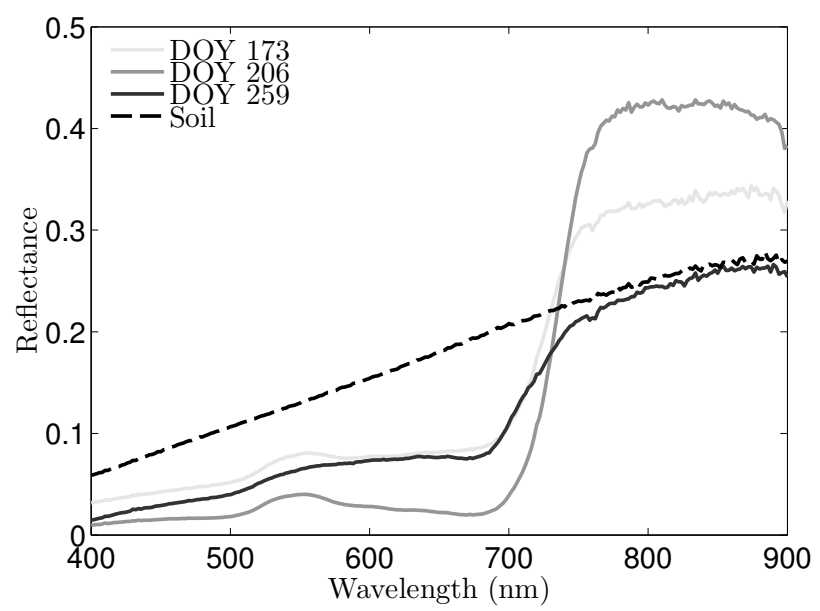

Figure 5.1: Canopy reflectance observations on DOY 173, 206 and 259 in the growing season and soil reflectance (DOY, day of year). 


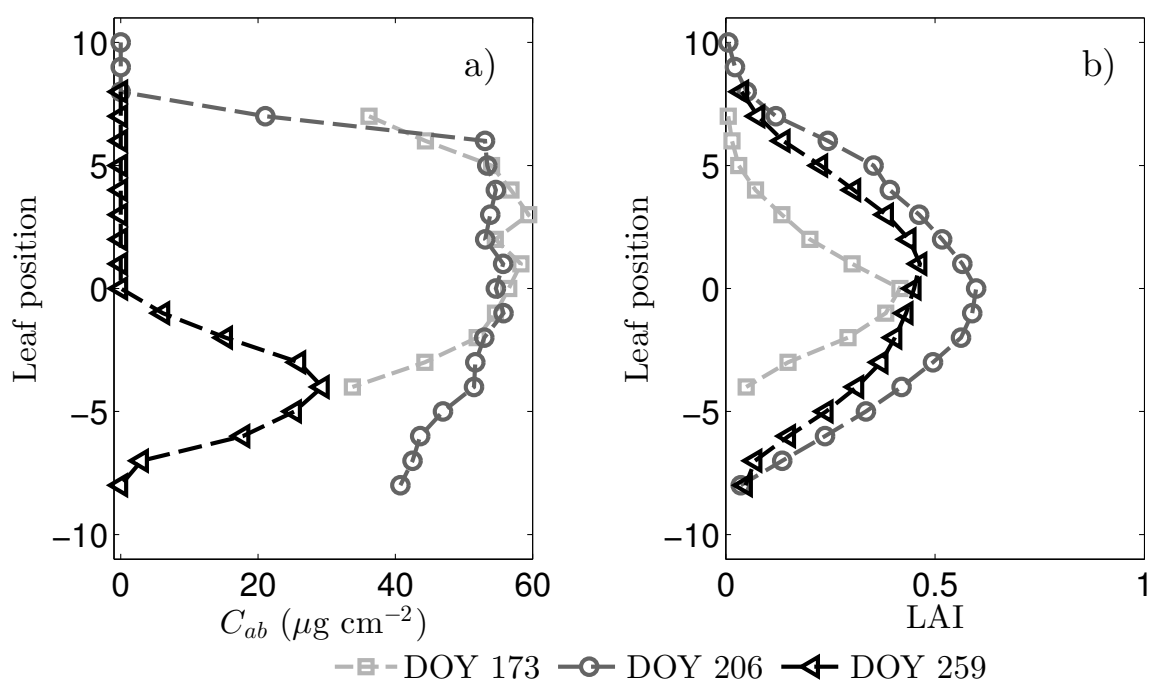

Figure 5.2: Vertical profile of leaf chlorophyll content $\left(C_{a b}\right)$ and LAI in the field datasets acquired on three days in the corn growing season. (Note, $y$ axis represents leaf position. The collar or ear leaf was labelled as leaf 0 . The leaves above or below leaf 0 were identified with a '+' or '-' sign, respectively, with the corresponding position number. For example, the first leaf above the collar or ear leaf was identified as +1 , the first leaf below the collar or ear leaf was identified as -1 ; DOY, day of year).

We first approximated the canopy structure and leaf properties from TOC reflectance by inverting the reflectance calculating routine in RTMo of SCOPE. The numerical optimization method (Nocedal and Wright, 2006) was used to retrieve the parameters $C_{a b}, C_{d m}, C_{w}, C_{s}, C_{c a}, N_{l}$, LIDFa and LIDFb by minimizing a cost function:

$$
C=\sum_{i=1}^{n}\left[R_{m}(i)-R_{s}(i)\right]^{2}
$$

where $R_{m}$ and $R_{s}$ were measured and simulated TOC reflectance, and $i$ represented a band in the reflectance measurements. In the retrieval, LAI was fixed to the measured canopy LAI, which was $1.63,5.23$ and 3.77 on the three days.

Reflectance, fluorescence and photosynthesis were simulated for each canopy from both SCOPE and mSCOPE. In the SCOPE simulation, the retrieved parameters were used except for chlorophyll content, for which 
canopy mean values measured $\left(50.2,41.2,7.8 \mu \mathrm{g} \mathrm{cm}^{-2}\right)$ were used. In the mSCOPE simulations, the measured leaf chlorophyll profiles and the retrieved canopy 'effective' values of other properties were used as input. The canopy had 12, 17 and 19 leaves on DOY 173, DOY 206 and DOY 259, respectively. Chlorophyll of each leaf was measured (Fig. 5.2). Regardless of the different vertical complexity of the corn canopy on three days, the vertical profiles of $C_{a b}$ of the canopy were implemented in mSCOPE in three ways, notably with 3, 7, and 11 layers. Leaves that had similar values of chlorophyll content were merged into one layer and the mean chlorophyll content of the leaves was assigned to the merged layer. Incident PAR was set to $1200 \mu \mathrm{mol} \mathrm{m} \mathrm{m}^{-2} \mathrm{~s}^{-1}$ as shown in Table 5.1.

\subsection{Evaluation and validation}

The simulation results of the six scenarios in the synthetic dataset were compared to evaluate how the vertical heterogeneity of chlorophyll and water content affects the canopy reflectance, fluorescence and photosynthesis. First, simulated TOC reflectance (nadir, 400-2400 nm) and TOC fluorescence (nadir, $650-850 \mathrm{~nm}$ ) from S1-S5 were visually compared to the homogeneous scenario S0. Then, one visible band (550 nm) and one near-infrared water absorption band $(1200 \mathrm{~nm})$ were selected for quantitative evaluation. Fluorescence at $687 \mathrm{~nm}$ and $760 \mathrm{~nm}\left(F_{687}\right.$ and $\left.F_{760}\right)$ were also compared, representing the red and far-red fluorescence used in remote sensing of vegetation (Meroni et al., 2009; Rascher et al., 2015). Further, simulations of net photosynthesis, absorbed photosynthetically active radiation (aPAR) and photosynthetic light use efficiency $(\mathrm{LUE}=A / \mathrm{aPAR})$ were compared and evaluated. SCOPE and mSCOPE were crossed validated by comparing their respective simulation results of the homogeneous scenario.

For the field corn dataset, simulations of TOC reflectance from mSCOPE were compared with the field measurements, to validate the accuracy of mSCOPE. Also, simulation results from mSCOPE were compared with results from SCOPE, to evaluate the effects of vertical distributions of $C_{a b}$ on TOC reflectance, fluorescence and canopy net photosynthesis. 


\section{Results}

\subsection{Simulation results of synthetic two-layer canopies}

TOC reflectance, TOC fluorescence and canopy photosynthesis of the six synthetic scenarios were simulated from mSCOPE. The results of the heterogeneous scenarios (S1-S5) and the comparison reference homogeneous scenario (S0) are shown in Fig. 5.3.
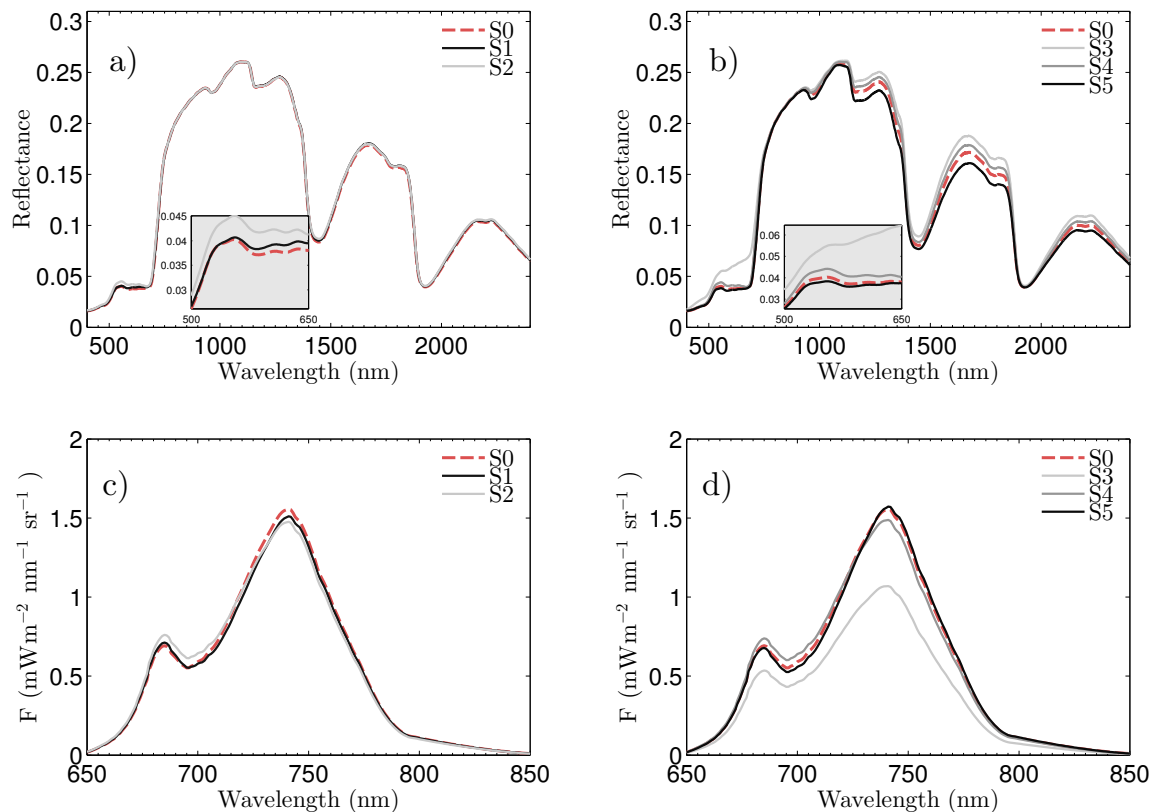

Figure 5.3: Simulation results for the six synthetic scenarios from mSCOPE a), b) nadir reflectance spectra; c), d) nadir fluorescence spectra (Note, S0 is a homogeneous scenario, S1-S5 have different vertical distribution of chlorophyll content $\left(C_{a b}\right)$ and leaf water content $\left.\left(C_{w}\right)\right)$. Reflectance spectra from 500 to $650 \mathrm{~nm}$ were enlarged in the grey boxes.

Modelled reflectance was different for each group-1 scenario (S0, S1 and S2), despite having equal total canopy $C_{a b}$ and $C_{w}$ (Fig. 5.3a). The difference was especially obvious in the visible region from 400 to $700 \mathrm{~nm}$. Modelled reflectance also varied significantly among group-2 scenarios (S0, S3, S4 and S5), where upper layer had the same $C_{a b}$ and $C_{w}$ while lower layer $C_{a b}$ and $C_{w}$ increased from S3 to S5. The differences were obvious in both the visible and infrared region (700-2400 nm). Especially, the disparity of infrared 
reflectance among group-2 scenarios was much higher than the disparity among group-1 scenarios. Simulated reflectance was clearly the highest in S3 among the group-2 scenarios. Reflectance at $550 \mathrm{~nm}$ of S3, S4 and S5 differed by $34 \%, 9 \%$ and $5 \%$ respectively, compared with the reflectance of the homogeneous canopy $\mathrm{S} 0$, and reflectance at $1200 \mathrm{~nm}$ differed by $2 \%, 2 \%$ and $6 \%$, respectively.

Fluorescence of the six scenarios also varied (Fig. 5.3c and 5.3d). In group-1 scenarios, at $687 \mathrm{~nm}\left(F_{687}\right), \mathrm{S} 1$ and $\mathrm{S} 2$ differed $3 \%$ and $10 \%$ respectively, compared to the homogeneous canopy S0; while at $760 \mathrm{~nm}\left(F_{760}\right)$, the difference of $\mathrm{S} 1, \mathrm{~S} 2$ with $\mathrm{S} 0$ is marginal. In group-2 scenarios, at $687 \mathrm{~nm}$ $\left(F_{687}\right), \mathrm{S} 3, \mathrm{~S} 4$, and S5 differed $23 \%, 7 \%$, and $2 \%$ respectively, compared to the homogeneous canopy S0; while at $760 \mathrm{~nm}\left(F_{760}\right)$, the difference to S0 was $33 \%, 6 \%$ and $2 \%$ respectively.

Table 5.4: Photosynthetically active radiation absorbed (aPAR), net photosynthesis $(A)$ and light use efficiency (LUE) simulated from mSCOPE of the six synthetic scenarios.

\begin{tabular}{lccc}
\hline Scenario & $\begin{array}{c}\mathrm{aPAR} \\
\left(\mu \mathrm{mol} \mathrm{m}^{-2} \mathrm{~s}^{-1}\right)\end{array}$ & $\begin{array}{c}A \\
\left(\mu \mathrm{mol} \mathrm{CO} 2 \mathrm{~m}^{-2} \mathrm{~s}^{-1}\right)\end{array}$ & $\begin{array}{c}\mathrm{LUE} \\
\left(\mathrm{mol} \mathrm{CO}_{2} \mathrm{~mol}^{-1} \text { photon }\right)\end{array}$ \\
\hline S0 & 943.02 & 25.17 & 0.027 \\
S1 & 973.07 & 25.07 & 0.026 \\
S2 & 842.40 & 24.78 & 0.030 \\
S3 & 788.28 & 20.47 & 0.026 \\
S4 & 922.50 & 24.85 & 0.027 \\
S5 & 951.64 & 25.28 & 0.027 \\
\hline
\end{tabular}

The results of net photosynthesis $(A)$, absorbed photosynthetically active radiation (aPAR) by all phyto-elements and light use efficiency (LUE $=A / \mathrm{aPAR}$ ) varied in the six scenarios (Table 5.4). In group-1 scenarios (S0, S1 and S2), canopies with the same total $C_{a b}$ and $C_{w}$ had a similar photosynthesis, but different aPAR and LUE. The S1 canopy absorbed more light than the S0 and S2 canopies, but had the smallest LUE. In group-2 scenarios (S0, S3, S4 and S5), aPAR and $A$ increased with increasing total $C_{a b}$, but differences among scenarios in LUE were minor. 


\subsection{Simulated and measured results of field corn canopy}

The retrievals of canopy structure and leaf properties from measured reflectance (Table 5.5) show that the relative senescence material $C_{s}$ of corn was 0 in the early and middle growing season (DOY 173, DOY 206), but 0.4 in the senescent stage (DOY 259). Both leaf water content $\left(C_{w}\right)$ and carotenoid content $\left(C_{c a}\right)$ were the lowest on DOY 259. The retrieved $C_{a b}$ was close to measured canopy mean $C_{a b}$ on DOY 173 and 206 (50.2 and 41.2 $\mu \mathrm{g} \mathrm{cm}^{-2}$ ), but it was three times as high as measured canopy mean $C_{a b}$ on DOY $259\left(7.5 \mu \mathrm{g} \mathrm{cm}^{-2}\right)$.

Table 5.5: The parameters of canopy structure and leaf properties of the corn canopy retrieved from the TOC reflectance measurements.

\begin{tabular}{llll}
\hline Parameter & DOY 173 & DOY 206 & DOY 259 \\
\hline$C_{d m}\left(\mathrm{~g} \mathrm{~cm}^{-2}\right)$ & 0.01 & 0.04 & 0.005 \\
$C_{w}(\mathrm{~cm})$ & 0.04 & 0.05 & 0.01 \\
$C_{s}$ & 0 & 0 & 0.4 \\
$N_{l}$ & 1.5 & 1.7 & 1.4 \\
$C_{c a}\left(\mu \mathrm{g} \mathrm{cm}^{-2}\right)$ & 5.6 & 3.7 & 1.4 \\
$C_{a b}\left(\mu \mathrm{g} \mathrm{cm}^{-2}\right)$ & 55 & 38 & 25 \\
LIDFa & -0.79 & -0.97 & -1 \\
LIDFb & 0.21 & 0.03 & 0 \\
\hline
\end{tabular}

The retrieved values (except $C_{a b}$ ) were further input into mSCOPE and SCOPE for forward simulations. Modelled TOC reflectance and fluorescence from mSCOPE and SCOPE, as well as measured TOC reflectance are presented in Fig. 5.4, and simulated photosynthesis in Table 5.6. SCOPE and $\mathrm{mSCOPE}$ (3, 7 or 11 layers) produced similar reflectance simulations, and they were all close to the measured reflectance on both DOY 173 and DOY 206. However, the simulations for DOY 259 diverged. Compared to SCOPE, mSCOPE performed much better in terms of reflectance simulation in the visible spectral region. Reflectance from SCOPE was clearly lower than reflectance from $\mathrm{mSCOPE}$ in the range of 570 to $700 \mathrm{~nm}$. However, fluorescence (650 to $850 \mathrm{~nm}$ ) from SCOPE was much higher than fluorescence from mSCOPE at DOY 259. The number of layers in mSCOPE had negligible impact on the modelling results. Simplification of the corn canopy into 3 , 7 or 11 layers, all produced similar fluorescence. Photosynthesis simulation with SCOPE and mSCOPE were very similar for DOY 173 and DOY 206. 

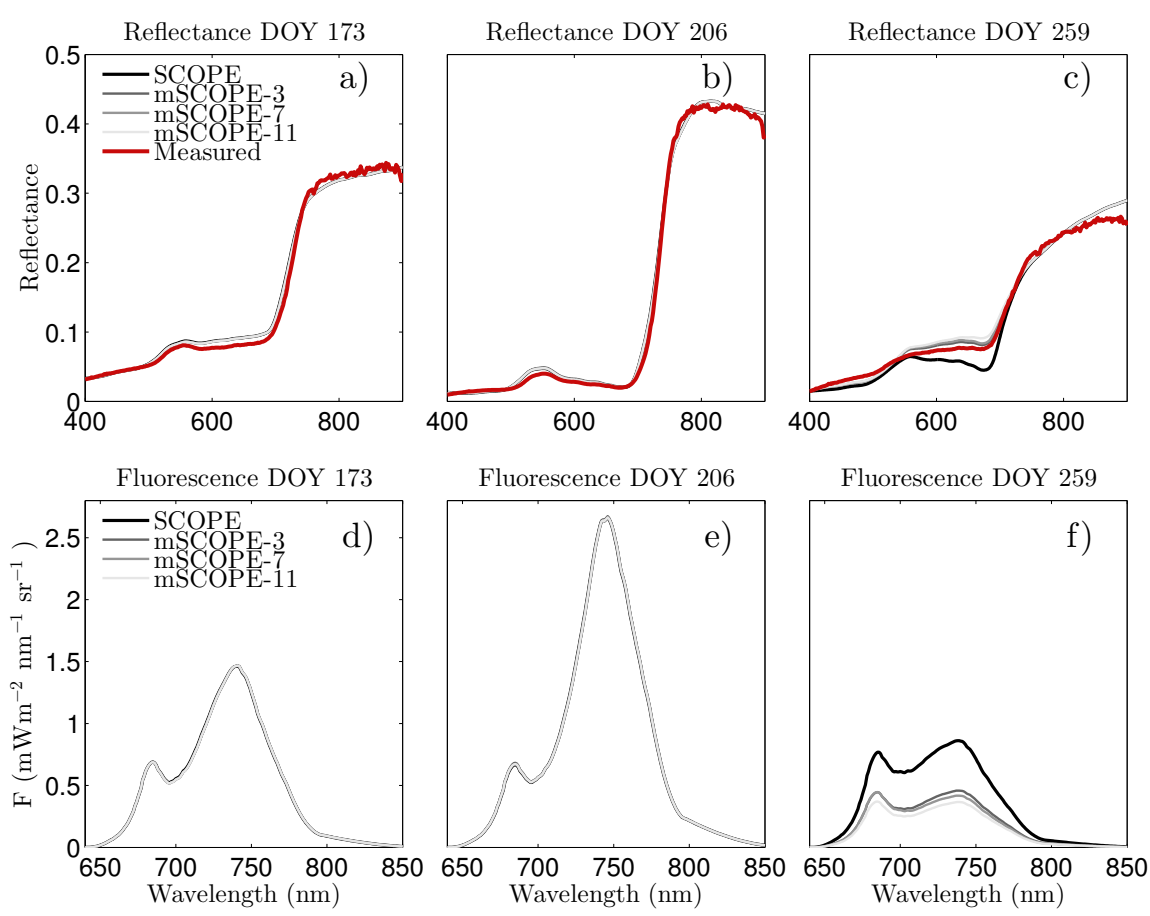

Figure 5.4: Simulation results of the corn canopy on three days in the growing season. upper panel: comparison among measured, mSCOPE modelled, and SCOPE modelled reflectance; lower panel: comparison between mSCOPE modelled and SCOPE modelled fluorescence (Note: the vertical profile of $C_{a b}$ for each canopy was simplified as 3,7 , and 11 layers and implemented in mSCOPE. DOY, day of year).

Howver, a notable difference was found in the results on DOY 259, when the homogeneous SCOPE model produced a much higher (more than three-fold) photosynthesis than mSCOPE.

Table 5.6: Net photosynthesis simulated from mSCOPE and SCOPE of the corn canopy on the three days.

\begin{tabular}{llll}
\hline & \multicolumn{3}{l}{ Photosynthesis $\left(\mu \mathrm{mol} \mathrm{CO}_{2} \mathrm{~m}^{-2} \mathrm{~s}^{-1}\right)$} \\
& DOY 173 & DOY 206 & DOY 259 \\
\hline SCOPE & 23.8 & 37.7 & 27.9 \\
mSCOPE-3 & 23.9 & 37.1 & 6.7 \\
mSCOPE-7 & 23.9 & 37.0 & 5.9 \\
mSCOPE-11 & 23.9 & 37.0 & 5.9 \\
\hline
\end{tabular}




\section{Discussion}

\subsection{Model validation}

The performance of mSCOPE has been tested in two ways: by analysing the differences with SCOPE, and by comparing simulated to measured reflectance of corn. For a homogeneous canopy, SCOPE and mSCOPE produce identical output (results not shown). If the vertical heterogeneity of the canopy is limited, such as in the corn canopy data used in this study on DOY 173 and DOY 206, then mSCOPE and SCOPE produce very similar reflectance, fluorescence and photosynthesis simulations.

\subsection{Effects of vertical heterogeneity on TOC reflectance, fluorescence and photosynthesis}

The modelled results from the six synthetic scenarios showed noticeable effect of vertical heterogeneity on canopy TOC reflectance, which confirms findings in previous modelling studies (Kuusk, 2001; Widlowski et al., 2007; Wang and Li, 2013; Zhao et al., 2017). Furthermore, significant effect of canopy heterogeneity on simulated TOC fluorescence and photosynthesis was demonstrated in this study (Fig. 5.3c, 5.3d). TOC reflectance and fluorescence vary with different vertical distribution of $C_{a b}$ and $C_{w}$, even if the canopies have the same total $C_{a b}$ and $C_{w}$ (Fig. 5.3a and 5.3c). The lower leaves in a canopy with $\mathrm{LAI}=3$ still has a noticeable impact on TOC reflectance and fluorescence (Fig. 5.3b and 5.3d). It is worth noting that canopy openness plays an important role in relative contribution of upper and leaves in the canopy signal (Martens et al., 1993; Chen and Cihlar, 1995; Nascimento et al., 2007). The lower leaves in canopies with low LAI have a better chance $\left(P_{o}\right.$ in Eq. 5.21) to be observed than those with high LAI.

The differences in fluorescence were mainly caused by the distribution of $C_{a b}$, and the effect of $C_{w}$ was very small. Different $C_{a b}$ distribution led to different aPAR (Jacquemoud et al., 2009), resulting in different fluorescence emission. The effects of vertical heterogeneity of leaf properties on the fluorescence appear to be wavelength-dependent. $F_{760}$ and $F_{687}$ varied differently 
among the synthetic scenarios (Fig. 5.3d). This wavelength dependence is caused by reabsorption: $F_{687}$ has higher re-absorption and lower scattering than $F_{760}$.

The results of the corn canopy demonstrate that neglecting vertical heterogeneity of $C_{a b}$ may lead to significant biases in prediction of TOC reflectance, fluorescence and canopy photosynthesis in the senescent stage. The biases of vegetation models as a result of simplifications depend on the complexity of the canopies in reality, which was also demonstrated for TOC reflectance by Wang and Li (2013). In the senescent stage of crops, canopies usually have large vertical heterogeneity of leaf properties (Verhoef and Bach, 2007; Ciganda et al., 2008; Liu et al., 2015). Compared to SCOPE, mSCOPE provided a closer prediction of TOC reflectance to the field measurement in the visible region due to the consideration of the vertical heterogeneity of $C_{a b}$ (Fig. 5.4c). SCOPE produced considerably higher estimates of both fluorescence and photosynthesis in the senescent stage when average $C_{a b}$ is used as input (Fig. 5.4f and Table 5.6) compared to mSCOPE. Although simulations of a multi-layer canopy are different from a single layer canopy, the number of layers does not need to be large: The three simplifications in mSCOPE (mSCOPE-3, mSCOPE-7 and mSCOPE-11) yield similar results. Thus, the three-layer simplification (mSCOPE-3) appears a sufficiently detailed representation of the vertical heterogeneity of the senescent corn, and adding layers does not affect the results significantly in this case. The fact that three layers is sufficient may be due to the 'bell-shape' profiles (Ciganda et al., 2008) of chlorophyll (Fig. 5.2), which can easily be approximated by three layers. However, a single layer as in SCOPE may be an oversimplification.

From the above analysis, the effects of considering canopy vertical heterogeneity on reflectance, fluorescence and photosynthesis has been demonstrated. However, our experimental setup was rather simple compared to complex canopies in reality. In the synthetic scenarios, two layer canopies were generated, and only $C_{a b}$ and $C_{w}$ were varied vertically. In the corn canopy, only vertical variation of $C_{a b}$ was considered. The lack of measurements of fluorescence and photosynthesis of the corn canopies also limited 
the validation of the full model. Since the main focus of this paper is to introduce the mSCOPE model, only a simplified validation and evaluation were conducted.

\subsection{Implications}

The model mSCOPE is an extension of SCOPE to include canopy vertical heterogeneity of leaf biophysical and biochemical properties. It simulates reflectance, fluorescence and photosynthesis of multi-layer canopies. Vegetation reflectance models for heterogeneous canopies have been developed. There are two-layer (Kuusk, 2001), multi-layer (Wang and Li, 2013) and 3-D models, such as DART (Gastellu-Etchegorry et al., 1996) and Raytran (Govaerts and Verstraete, 1998). DART recently included the fluorescence simulation (Gastellu-Etchegorry et al., 2017) to promote a better understanding of remote sensed signals and plant physiology. The model mSCOPE benefits from the original SCOPE model which incorporates the energy balance model. The energy balance model allows predicting the response of vegetation to the ambient conditions, such as temperature and humidity (Van der Tol et al., 2014).

The adding concept applied in $\mathrm{mSCOPE}$ has potential in radiative transfer modelling. It was originally used for the calculation of TOC reflectance (Cooper et al., 1982; Verhoef, 1985). We have extended the adding method to the calculation of vertical flux profiles incorporating the radiation emission (fluorescence) in multi-layer canopies. These have been summarized in Eq. 5.18, 5.18 and in Eq. 5.38, 5.39 (with fluorescence emission). Our approach can be applied in mediums other than vegetation, such as water and atmosphere which have a clear multi-layer structure. Moreover, it can also be used for the calculation of thermal fluxes in various mediums as similar to the calculation of fluorescence fluxes. The detailed mathematical explanation in section 2 will allow applying our approach in the potential applications mentioned above. 


\section{Conclusion}

Vegetation canopies generally exhibit vertical heterogeneity in leaf properties. Homogeneous models are in some cases insufficient in their representation of the canopy for understanding the remote sensing signal of reflectance, fluorescence and canopy photosynthesis. An integrated model of radiative transfer and energy balance that addresses vertical heterogeneity of leaf biophysical and biochemical parameters within the canopy has been proposed. The model mSCOPE simulates TOC reflectance, fluorescence and photosynthesis, for vertically heterogeneous canopies. It could provide a better understanding of remote sensing signals and plant physiology.

\section{Appendices}

\section{Extinction, scattering and emission coefficients}

Leaf reflectance and transmittance ( $\rho$ and $\tau$ ) are simulated by leaf RTMs (i.e., FLUSPECT in mSCOPE). The reflectance of both the adaxial and abaxial sides of leaves is assumed to the same in the model. Leaf angle is characterized by leaf zenith angle $\left(\theta_{l}\right)$ and azimuth angle $\left(\varphi_{l}\right)$. Numerically, 13 discrete leaf inclinations are used as in mSCOPE inherited from SCOPE and SAIL, and the uniform leaf azimuth distribution is now also discretized to 36 angles of $5,15, \ldots, 355^{\circ}$ relative to solar azimuth. The leaf inclination distribution is described by using mathematical functions (LIDF) (De Wit, 1965) which quantifies the probability of each leaf orientation class. Sunobserver geometry is determined by the sun and observer's zenith angle $\left(\theta_{s}\right.$ and $\left.\theta_{o}\right)$, and the relative azimuth angle $(\psi$, the absolute difference between 
their azimuth angles). The coefficients are expressed as follows:

$$
\begin{aligned}
f_{s} & =\cos \theta_{l}+\tan \theta_{s} \sin \theta_{l} \cos \varphi_{l} \\
f_{o} & =\cos \theta_{l}+\tan \theta_{o} \sin \theta_{l} \cos \left(\varphi_{l}-\psi\right) \\
k & =\left|f_{s}\right| \\
K & =\left|f_{o}\right| \\
s & =\frac{1}{2}\left[\left|f_{s}\right|(\rho+\tau)+f_{s} \cos \theta_{l}(\rho-\tau)\right] \\
s^{\prime} & =\frac{1}{2}\left[\left|f_{s}\right|(\rho+\tau)-f_{s} \cos \theta_{l}(\rho-\tau)\right] \\
\sigma & =\frac{1}{2}\left[(\rho+\tau)+(\rho-\tau) \cos \theta_{l}\right] \\
a & =1-\frac{1}{2}\left[(\rho+\tau)-(\rho-\tau) \cos ^{2} \theta_{l}\right] \\
v & =\frac{1}{2}\left[\left|f_{o}\right|(\rho+\tau)+f_{o} \cos \theta_{l}(\rho-\tau)\right] \\
v^{\prime} & =\frac{1}{2}\left[\left|f_{o}\right|(\rho+\tau)-f_{o} \cos \theta_{l}(\rho-\tau)\right] \\
w & =\frac{1}{2}\left[\left|f_{s} f_{o}\right|(\rho+\tau)+f_{s} f_{o}(\rho-\tau)\right] .
\end{aligned}
$$

The nine emission coefficients (those with subscripts of $f$ ) are determined both by sun-leaf-observer geometry, which is characterized by the zenith angles of the sun $\left(\theta_{s}\right)$, leaf $\left(\theta_{l}\right)$ and observer $\left(\theta_{o}\right)$, the leaf azimuth angle $\left(\varphi_{l}\right)$ and the relative azimuth angles between the sun and the observer $(\psi)$, and by the leaf excitation-emission matrices at the backward and forward side 
$\left(M_{b}\right.$ and $\left.M_{f}\right)$ (Vilfan et al., 2016). For individual leaves, they are given by

$$
\begin{aligned}
f_{s} & =\cos \theta_{l}+\tan \theta_{s} \sin \theta_{l} \cos \varphi_{l} \\
f_{o} & =\cos \theta_{l}+\tan \theta_{o} \sin \theta_{l} \cos \left(\varphi_{l}-\psi\right) \\
s_{f} & =\frac{1}{2}\left[\left|f_{s}\right|\left(M_{b}+M_{f}\right)+f_{s} \cos \theta_{l}\left(M_{b}-M_{f}\right)\right] \\
s_{f}^{\prime} & =\frac{1}{2}\left[\left|f_{s}\right|\left(M_{b}+M_{f}\right)-f_{s} \cos \theta_{l}\left(M_{b}-M_{f}\right)\right] \\
\sigma_{f} & =\frac{1}{2}\left[\left(M_{b}+M_{f}\right)+\left(M_{b}-M_{f}\right) \cos ^{2} \theta_{l}\right] \\
\sigma_{f}^{\prime} & =\frac{1}{2}\left[\left(M_{b}+M_{f}\right)-\left(M_{b}-M_{f}\right) \cos ^{2} \theta_{l}\right] \\
v_{f} & =\frac{1}{2}\left[\left|f_{o}\right|\left(M_{b}+M_{f}\right)+f_{o} \cos \theta_{l}\left(M_{b}-M_{f}\right)\right] \\
v_{f}^{\prime} & =\frac{1}{2}\left[\left|f_{o}\right|\left(M_{b}+M_{f}\right)-f_{o} \cos \theta_{l}\left(M_{b}-M_{f}\right)\right] \\
w_{f} & =\frac{1}{2}\left[\left|f_{s} f_{o}\right|\left(M_{b}+M_{f}\right)+f_{s} f_{o}\left(M_{b}-M_{f}\right)\right] .
\end{aligned}
$$

The excitation-emission matrices $\left(M_{b}\right.$ and $\left.M_{f}\right)$ are functions of fluorescence emission efficiency and thus are determined by the net radiation. It is noted that the matrices are dependent on the illumination conditions of the individual leaf.

\section{Effective downward transmittance $X$}

$$
\begin{aligned}
I-r_{b} R & =\left[\begin{array}{ll}
1 & 0 \\
0 & 1
\end{array}\right]-\left[\begin{array}{cc}
0 & 0 \\
\rho_{d d} & 0
\end{array}\right]\left[\begin{array}{ll}
R_{s d} & R_{d d} \\
R_{s o} & R_{d o}
\end{array}\right] \\
& =\left[\begin{array}{ll}
1 & 0 \\
0 & 1
\end{array}\right]-\left[\begin{array}{cc}
0 & 0 \\
\rho_{d d} R_{s d} & \rho_{d d} R_{d d}
\end{array}\right] \\
& =\left[\begin{array}{cc}
1 & 0 \\
-\rho_{d d} R_{s d} & 1-\rho_{d d} R_{d d}
\end{array}\right]
\end{aligned}
$$

Therefore, we find

$$
\left(I-r_{b} R\right)^{-1}=\left(1-\rho_{d d} R_{d d}\right)^{-1}\left[\begin{array}{cc}
1-\rho_{d d} R_{d d} & 0 \\
\rho_{d d} R_{s d} & 1
\end{array}\right]
$$


Post-multiplication by $t_{d}$ then gives

$$
\begin{aligned}
X & =\left(I-r_{b} R\right)^{-1} t_{d} \\
& =\left(1-\rho_{d d} R_{d d}\right)^{-1}\left[\begin{array}{cc}
1-\rho_{d d} R_{d d} & 0 \\
\rho_{d d} R_{s d} & 1
\end{array}\right]\left[\begin{array}{cc}
\tau_{s s} & 0 \\
\tau_{s d} & \tau_{d d}
\end{array}\right] \\
& =\left[\begin{array}{cc}
\tau_{s s} & 0 \\
\frac{\tau_{s d}+\tau_{s s} R_{s d} \rho_{d d}}{1-\rho_{d d} R_{d d}} & \frac{\tau_{d d}}{1-\rho_{d d} R_{d d}}
\end{array}\right]
\end{aligned}
$$

where only $R_{s d}$ and $R_{d d}$ refer to thick layer extending from the bottom to level $j$, which means that one can write

$$
X(j)=\left[\begin{array}{cc}
\tau_{s s}(j) & 0 \\
\frac{\tau_{s d}(j)+\tau_{s s}(j) R_{s d}(j) \rho_{d d}(j)}{1-\rho_{d d}(j) R_{d d}(j)} & \frac{\tau_{d d}(j)}{1-\rho_{d d}(j) R_{d d}(j)}
\end{array}\right]=\left[\begin{array}{cc}
X_{s s}(j) & 0 \\
X_{s d}(j) & X_{d d}(j)
\end{array}\right]
$$

\section{Bi-directional gap probability}

For sublayer $j$, the vertical relatively coordinate is given by

$$
x=(j-60) / 60
$$

and the bi-directional gap probability of the sublayer is given by Verhoef (1998)

$$
P_{s o}(j)=\exp \left\{(K+k) x+\sqrt{K k} \frac{s_{l}}{a}\left[1-\exp \left(x a / s_{l}\right)\right]\right\}
$$

where $s_{l}$ is the hot spot size parameter, approximated as

$$
s_{l}=\frac{w_{l}}{h} \frac{2}{K+k}
$$

where $w_{l}$ is the average leaf width and $h$ canopy height, and the factor $2 /(K+k)$ accomplishes a correction for leaf projection area on a horizontal plane. The function $a$ depends only on the sun-target-sensor angular geometry, and is given by

$$
a=\sqrt{\tan ^{2} \theta_{s}+\tan ^{2} \theta_{o}-2 \tan \theta_{s} \tan \theta_{o} \cos \psi}
$$


Concluding remarks and prospects

6 
Monitoring terrestrial vegetation photosynthesis is a key to understand and predict carbon dynamics and the adaptation of ecosystem functions to environmental changes. Remote sensing of sun-induced chlorophyll fluorescence (SIF) is a promising way to monitor vegetation photosynthesis. With the increased availability of SIF data, the use of SIF-based approaches is increasing to obtain an insight into the functional aspects of plant communities. Three processes determine SIF observed by remote sensors: photosynthetic light absorption, conversion of absorbed light to fluorescence, re-absorption and scattering of emitted fluorescence. It is essential to separate these three quantitatively to move beyond the mere empirical observation of the SIF-photosynthesis relationship.

\section{Conclusions and implications}

\subsection{Summary of conclusions}

The key to using SIF is not to use it as a proxy of photosynthesis directly, but to use it together with the reflectance spectrum. Only then the effects of APAR, physiology and scattering on SIF can be separately quantified. While radiative transfer models can serve for this purpose (Chapter 2), it is also possible to derive a relationship between the scattering of fluorescence and reflectance analytically (Chapter 3$)$, as:

$$
\sigma_{F}=\frac{R_{n i r}}{i_{0} \omega}
$$

This relationship explains the similarity between the viewing angle dependence of SIF and reflectance reported in the literature. Moreover, it provides a way to correct the process of scattering of emitted SIF that is unrelated to photosynthesis, and allows downscaling TOC SIF to canopy emitted SIF.

In Chapter 4, combining the relationship found in Chapter 3 and a new model for fPAR (Eq. 6.2), a simple reflectance index (FCVI, fluorescence correction vegetation index) is proposed (Eq. 6.3).

$$
\mathrm{fPAR}=i_{0} \frac{R_{\text {nir }}-R_{\text {vis }}}{R_{\text {nir }}}
$$




$$
\mathrm{FCVI}=R_{\text {nir }}-R_{\text {vis }}
$$

FCVI as a difference between near-infrared and broadband visible (400 - $700 \mathrm{~nm}$ ) reflectance quantifies the effects of light absorption and SIF re-absorption (and scattering) on observed SIF signals. It allows downscaling TOC SIF to fluorescence emission efficiency. Applying this index to GOME-2 SIF measurements, global fluorescence emission efficiency maps are obtained.

In Chapter 5, a model for light interaction and energy balance in multilayer canopies is developed. The mSCOPE model simulates SIF, reflectance and photosynthesis in multilayer vegetation canopies. The modelling approaches described can be used in radiative transfer of emitted SIF, scattered radiation and thermal radiation in water, vegetation and atmosphere. This adaptation of SCOPE is particularly useful in vegetation with a strong vertical gradient of pigments, such as senescent vegetation.

\subsection{Implications}

The approaches proposed and models developed in this thesis are expected to play an important role in a better interpretation of SIF signals for remote sensing of photosynthesis. By accounting for scattering effects, TOC SIF is converted to canopy emitted SIF. By further consideration of photosynthetic light absorption, fluorescence emission efficiency is retrieved. The correction of scattering effects on SIF yields canopy fluorescence production, which is directly linked with GPP. The correction of non-physiological regulation of SIF yields fluorescence emission efficiency, which is directly linked with photosynthetic efficiency. This can both be done with RTMs (Chapter 2) and with the analytical function and index (Chapter 3 and 4). It is worth noting that the RTM-based approach in Chapter 2 quantifies the three process over the whole spectral region (i.e. 640 - $850 \mathrm{~nm}$ ), while the approaches in Chapter 3 and 4 are developed and valid only for far-red SIF.

This thesis demonstrates that canopy reflectance is essential to estimate the effects of canopy structure, leaf properties and observational condition 
on SIF and disentangle physiological regulation of SIF. Both the RTM-based approaches (Chapter 2) and the approaches in Chapter 3 and 4 rely on reflectance data. In the RTM-based approaches reflectance allows retrieving of vegetation variables by inverting RTMs which can be used to predict light absorption and scattering of SIF. In Chapter 3 and 4, both scattering of SIF $\left(\sigma_{F}\right.$, for far-red SIF) and photosynthetic light absorption (fPAR) are directly approximated by using simple functions of canopy reflectance.

Radiative transfer models are important tools for the interpretation of optical remote sensing signals (e.g. reflectance and fluorescence). They provide the physical relations among remotely sensed signals, vegetation properties and observational conditions. By means of RTMs one is able to explain the relationship between canopy structure, leaf properties and sun-observer geometry and canopy reflectance. RTMs of fluorescence provide exciting opportunities for the quantitative use of SIF signals. The inclusion of SIF in the leaf RTMs connects fluorescence at photosynthetic level and at leaf level. Canopy SIF RTMs further connects leaf-level fluorescence to SIF actually observed from remote sensing techniques. The SCOPE and mSCOPE model can serve as a tool to better understand reflectance and SIF signals.

\section{Further challenges on the way ahead}

Several technical and scientific challenges were encountered, but not resolved in this thesis. First, the RTM-based approach provides explicit qualification of the photosynthetic light absorption and fluorescence re-absorption process, but it is computationally more expensive because of the necessary retrieval of vegetation variables from reflectance by using model inversion. Moreover, the retrieval of vegetation variables from reflectance is sometimes ill-posed and the uncertainty has not been fully quantified. Second, the method of using reflectance for scattering of SIF only works in the nearinfrared region, but not in the red region. The asymmetric distribution of scattered radiation and emitted SIF radiation over the two sides of a leaf in the red region is the main cause of the complex relationship of scattering of 
SIF and reflectance. The comparison of distribution of scattered radiation and emitted SIF radiation relies on the understanding of radiative transfer within a leaf. Further studies on leaf radiative transfer may provide a complete picture of the scattering effect on red SIF. Alternative option is generating an empirical relationship between within-leaf scattering of SIF and leaf reflectance and transmittance from leaf-level measurements, and upscaling the relationship to canopy through radiative transfer.

Several approaches have been proposed and discussed to interpret SIF measurements, but SIF has not been yet connected with photosynthesis mechanistically. We have moved beyond empirical relationships by quantitatively separating non-physiological and physiological regulation mechanisms on TOC SIF. This implies that the total emitted SIF of a canopy and fluorescence emission efficiency of photosystems can be obtained from TOC SIF observations. The next question that has not been answered in this thesis, is how fluorescence emission efficiency is related to photosynthetic efficiency. In this respect, we are still at the point of Van der Tol et al. (2014), namely an empirical model. Mechanistically linking fluorescence emission efficiency with photosynthetic efficiency is the biggest challenge ahead.

One possible way is to independently measure NPQ (heat dissipation) from reflectance. It is well-known that dynamic NPQ associates with changes in carotenoid pigments (e.g. xanthophyll pigments) in live foliage. Moreover, it is evident that NPQ regulations affect visible reflectance. Therefore, it may be possible to estimate NPQ or even the efficiency of heat dissipation from reflectance data. For example, PRI computed as the normalized difference of reflectance at $531 \mathrm{~nm}$ and $570 \mathrm{~nm}$ has been shown to be correlated with NPQ. Quantification of the efficiency of heat dissipation requires more mechanistic approaches rather than the semi-empirical indices. Modelling efforts to link NPQ and visible reflectance have been made (Vilfan et al., 2018). The first attempt on estimating NPQ from reflectance spectra is ongoing. It is promising to retrieve the efficiency of heat dissipation from reflectance data. With fluorescence emission efficiency retrieved from TOC SIF measurements, one may eventually quantify photosynthetic efficiency from remote sensing 
data.

Another option is looking at the dynamic response of SIF to climatic variables, notably temperature and light intensities. Both SIF and photosynthesis respond to ambient environmental conditions dynamically. Their responses to certain environmental factors (e.g. light intensity and temperature) have specific patterns. By looking into the patterns in the response of fluorescence, one may be able to reveal photosynthetic traits. For example, the light saturation point in the fluorescence-light response curve is closely related to the maximum rate of carboxylation (see Fig. 4. 9). With the increase of high temporal resolution SIF data, we can fully explore the potential of SIF.

In conclusion, this thesis provides several ways to interpret TOC SIF measurements and solve several problems in a remote sensing perspective. With the future work on the mechanistic link between fluorescence and photosynthesis, it is hoped that one can quantitatively monitor photosynthesis at regional and global scales from remote sensing data. 


\section{Bibliography}

Ač, A., Malenovskỳ, Z., Olejníčková, J., Gallé, A., Rascher, U., Mohammed, G., 2015. Meta-analysis assessing potential of steady-state chlorophyll fluorescence for remote sensing detection of plant water, temperature and nitrogen stress. Remote sensing of environment 168, 420-436.

Ainsworth, E. A., Long, S. P., 2005. What have we learned from 15 years of free-air CO2 enrichment (FACE)? A meta-analytic review of the responses of photosynthesis, canopy properties and plant production to rising CO2. New Phytologist 165 (2), 351-372.

Alonso, L., Gomez-Chova, L., Vila-Frances, J., Amoros-Lopez, J., Guanter, L., Calpe, J., Moreno, J., 2008. Improved Fraunhofer Line Discrimination method for vegetation fluorescence quantification. IEEE Geoscience and Remote Sensing Letters 5 (4), 620-624.

Amorós-López, J., Gomez-Chova, L., Vila-Frances, J., Alonso, L., Calpe, J., Moreno, J., del Valle-Tascon, S., 2008. Evaluation of remote sensing of vegetation fluorescence by the analysis of diurnal cycles. International Journal of Remote Sensing 29 (17-18), 5423-5436.

Asrar, G., Kanemasu, E. T., Miller, G. P., Weiser, R., 1986. Light interception and leaf area estimates from measurements of grass canopy reflectance. IEEE Transactions on Geoscience and Remote Sensing (1), 76-82.

Badgley, G., Field, C. B., Berry, J. A., 2017. Canopy near-infrared reflectance and terrestrial photosynthesis. Science Advances 3 (3), e1602244. 
Baker, N. R., 2008. Chlorophyll fluorescence: a probe of photosynthesis in vivo. Annu. Rev. Plant Biol. 59, 89-113.

Baldocchi, D., Falge, E., Gu, L., Olson, R., Hollinger, D., Running, S., Anthoni, P., Bernhofer, C., Davis, K., Evans, R., et al., 2001. FLUXNET: A new tool to study the temporal and spatial variability of ecosystem-scale carbon dioxide, water vapor, and energy flux densities. Bulletin of the American Meteorological Society 82 (11), 2415-2434.

Baldocchi, D. D., 2003. Assessing the eddy covariance technique for evaluating carbon dioxide exchange rates of ecosystems: past, present and future. Global change biology 9 (4), 479-492.

Baret, F., Guyot, G., 1991. Potentials and limits of vegetation indices for LAI and APAR assessment. Remote sensing of environment 35 (2-3), 161-173.

Barriopedro, D., Fischer, E. M., Luterbacher, J., Trigo, R. M., García-Herrera, R., 2011. The hot summer of 2010: Redrawing the temperature record map of Europe. Science 332 (6026), 220-224.

Bartlett, D. S., Whiting, G. J., Hartman, J. M., 1989. Use of vegetation indices to estimate indices to estimate intercepted solar radiation and net carbon dioxide exchange of a grass canopy. Remote Sensing of Environment $30(2), 115-128$.

Barton, C. V. M., North, P., 2001. Remote sensing of canopy light use efficiency using the photochemical reflectance index: Model and sensitivity analysis. Remote Sensing of Environment 78 (3), 264-273.

Bazzaz, F. A., 1990. The response of natural ecosystems to the rising global CO2 levels. Annual review of ecology and systematics 21 (1), 167-196.

Beniston, M., 2004. The 2003 heat wave in Europe: A shape of things to come? An analysis based on Swiss climatological data and model simulations. Geophysical Research Letters 31 (2).

Berk, A., Anderson, G. P., Acharya, P. K., Bernstein, L. S., Muratov, L., Lee, J., Fox, M., Adler Golden, S. M., Chetwynd, J. H., Hoke, M. L., 
et al., 2005. MODTRAN 5: a reformulated atmospheric band model with auxiliary species and practical multiple scattering options: update. En: Defense and Security. International Society for Optics and Photonics, pp. $662-667$.

Berry, J., Bjorkman, O., 1980. Photosynthetic response and adaptation to temperature in higher plants. Annual Review of plant physiology 31 (1), $491-543$.

Berry, J. A., 2012. There ought to be an equation for that. Annual review of plant biology $63,1-17$.

Biskup, B., Scharr, H., Schurr, U., Rascher, U., 2007. A stereo imaging system for measuring structural parameters of plant canopies. Plant, cell \& environment 30 (10), 1299-1308.

Björkman, O., Powles, S. B., 1984. Inhibition of photosynthetic reactions under water stress: interaction with light level. Planta 161 (6), 490-504.

Bloom, A. J., Chapin III, F. S., Mooney, H. A., 1985. Resource limitation in plants-an economic analogy. Annual review of Ecology and Systematics $16(1), 363-392$.

Carter, G. A., Miller, R. L., 1994. Early detection of plant stress by digital imaging within narrow stress-sensitive wavebands. Remote Sensing of Environment 50 (3), 295-302.

Cendrero-Mateo, M. P., Moran, M. S., Papuga, S. A., Thorp, K., Alonso, L., Moreno, J., Ponce-Campos, G., Rascher, U., Wang, G., 2015. Plant chlorophyll fluorescence: active and passive measurements at canopy and leaf scales with different nitrogen treatments. Journal of Experimental Botany 67 (1), 275-286.

Chaves, M. M., Pereira, J. S., Maroco, J., Rodrigues, M. L., Ricardo, C. P. P., Osório, M. L., Carvalho, I., Faria, T., Pinheiro, C., 2002. How plants cope with water stress in the field? photosynthesis and growth. Annals of botany 89 (7), 907-916. 
Chen, J. M., Cihlar, J., 1995. Plant canopy gap-size analysis theory for improving optical measurements of leaf-area index. Applied optics 34 (27), $6211-6222$

Chongya Jiang, H. F., 2012. Modeling soil reflectance using a global spectral library. 3-7, December, AGU Fall Meeting, San Francisco.

Christidis, N., Jones, G. S., Stott, P. A., 2015. Dramatically increasing chance of extremely hot summers since the 2003 European heatwave. Nature Climate Change 5 (1), 46-50.

Ciais, P., Reichstein, M., Viovy, N., Granier, A., Ogée, J., Allard, V., Aubinet, M., Buchmann, N., Bernhofer, C., Carrara, A., et al., 2005. Europe-wide reduction in primary productivity caused by the heat and drought in 2003 . Nature 437 (7058), 529-533.

Ciganda, V., Gitelson, A., Schepers, J., 2008. Vertical profile and temporal variation of chlorophyll in maize canopy: Quantitative crop vigor indicator by means of reflectance-based techniques. Agronomy Journal 100 (5), 1409-1417.

Collatz, G. J., Ribas Carbo, M., Berry, J., 1992. Coupled photosynthesisstomatal conductance model for leaves of C4 plants. Functional Plant Biology 19 (5), 519-538.

Cooper, K., Smith, J., Pitts, D., 1982. Reflectance of a vegetation canopy using the adding method. Applied Optics 21 (22), 4112-4118.

Damm, A., Guanter, L., Laurent, V., Schaepman, M., Schickling, A., Rascher, U., 2014. FLD-based retrieval of sun-induced chlorophyll fluorescence from medium spectral resolution airborne spectroscopy data. Remote Sensing of Environment 147, 256-266.

Damm, A., Guanter, L., Paul-Limoges, E., Van der Tol, C., Hueni, A., Buchmann, N., Eugster, W., Ammann, C., Schaepman, M., 2015a. Far-red sun-induced chlorophyll fluorescence shows ecosystem-specific relationships 
to gross primary production: An assessment based on observational and modeling approaches. Remote Sensing of Environment 166, 91-105.

Damm, A., Guanter, L., Verhoef, W., Schläpfer, D., Garbari, S., Schaepman, M., 2015b. Impact of varying irradiance on vegetation indices and chlorophyll fluorescence derived from spectroscopy data. Remote sensing of environment 156, 202-215.

Darvishzadeh, R., Skidmore, A., Schlerf, M., Atzberger, C., 2008. Inversion of a radiative transfer model for estimating vegetation lai and chlorophyll in a heterogeneous grassland. Remote Sensing of Environment 112 (5), 2592-2604.

Daumard, F., Goulas, Y., Champagne, S., Fournier, A., Ounis, A., Olioso, A., Moya, I., 2012. Continuous monitoring of canopy level sun-induced chlorophyll fluorescence during the growth of a sorghum field. IEEE Transactions on Geoscience and Remote Sensing 50 (11), 4292-4300.

Davidson, M., Berger, M., Moya, I., Moreno, J., Laurila, T., Stoll, M.-P., Miller, J., 11 2003. Mapping photosynthesis from space - a new vegetationfluorescence technique 116, 34-37.

De Graaff, M.-A., Van Groenigeng, K.-J., Six, J., Hungate, B., van Kessel, C., 2006. Interactions between plant growth and soil nutrient cycling under elevated co2: A meta-analysis. Global Change Biology 12 (11), 2077-2091.

De Wit, C., 1962. Space relationships within populations of one or more species.

De Wit, C. T., 1965. Photosynthesis of leaf canopies. Agricultural Research Report, Pudoc.

Dobrowski, S., Pushnik, J., Zarco-Tejada, P., Ustin, S., 2005. Simple reflectance indices track heat and water stress-induced changes in steady-state chlorophyll fluorescence at the canopy scale. Remote Sensing of Environment 97 (3), 403-414. 
Dong, B., Sutton, R., Shaffrey, L., Wilcox, L., 2016. The 2015 European heat wave. Bulletin of the American Meteorological Society 97 (12), S57-S62.

Drake, B. G., Gonzàlez-Meler, M. A., Long, S. P., 1997. More efficient plants: a consequence of rising atmospheric CO2? Annual review of plant biology $48(1), 609-639$.

Dreccer, M., Van Oijen, M., Schapendonk, A., Pot, C., Rabbinge, R., 2000. Dynamics of vertical leaf nitrogen distribution in a vegetative wheat canopy. Impact on canopy photosynthesis. Annals of Botany 86 (4), 821-831.

Drusch, M., Moreno, J., del Bello, U., Franco, R., Goulas, Y., Huth, A., Kraft, S., Middleton, E., Miglietta, F., Mohammad, G., et al., 2016. The Fluorescence EXplorer (FLEX) Mission Concept-ESA's Earth Explorer 8 (EE8). IEEE Trans. Geosci. Rem. Sens.(TGRS).

Drusch, M., Moreno, J., Del Bello, U., Franco, R., Goulas, Y., Huth, A., Kraft, S., Middleton, E. M., Miglietta, F., Mohammed, G., et al., 2017. The FLuorescence EXplorer Mission Concept-ESA's Earth Explorer 8. IEEE Transactions on Geoscience and Remote Sensing 55 (3), 1273-1284.

Ehleringer, J., Björkman, O., 1977. Quantum yields for CO2 uptake in C3 and $\mathrm{C} 4$ plants: dependence on temperature, $\mathrm{CO} 2$, and $\mathrm{O} 2$ concentration. Plant Physiology 59 (1), 86-90.

Fan, W., Liu, Y., Xu, X., Chen, G., Zhang, B., 2014. A new FAPAR analytical model based on the law of energy conservation: A case study in china. IEEE Journal of Selected Topics in Applied Earth Observations and Remote Sensing 7 (9), 3945-3955.

Farquhar, G. D., Lloyd, J., Taylor, J. A., Flanagan, L. B., Syvertsen, J. P., Hubick, K. T., Wong, S. C., Ehleringer, J. R., 1993. Vegetation effects on the isotope composition of oxygen in atmospheric CO2. Nature 363 (6428), 439. 
Farquhar, G. v., Caemmerer, S. v., Berry, J., 1980. A biochemical model of photosynthetic CO2 assimilation in leaves of C3 species. Planta 149 (1), 78-90.

Field, C. B., Randerson, J. T., Malmström, C. M., 1995. Global net primary production: combining ecology and remote sensing. Remote Sensing of Environment 51 (1), 74-88.

Fournier, A., Daumard, F., Champagne, S., Ounis, A., Goulas, Y., Moya, I., 2012. Effect of canopy structure on sun-induced chlorophyll fluorescence. ISPRS journal of photogrammetry and remote sensing 68, 112-120.

Foyer, C. H., Ruban, A. V., Nixon, P. J., 2017. Photosynthesis solutions to enhance productivity.

Frankenberg, C., Fisher, J. B., Worden, J., Badgley, G., Saatchi, S. S., Lee, J.-E., Toon, G. C., Butz, A., Jung, M., Kuze, A., et al., 2011. New global observations of the terrestrial carbon cycle from GOSAT: Patterns of plant fluorescence with gross primary productivity. Geophysical Research Letters $38(17)$.

Frankenberg, C., O’Dell, C., Berry, J., Guanter, L., Joiner, J., Köhler, P., Pollock, R., Taylor, T. E., 2014. Prospects for chlorophyll fluorescence remote sensing from the orbiting Carbon Observatory-2. Remote Sensing of Environment 147, 1-12.

Frouin, R., Lingner, D. W., Gautier, C., Baker, K. S., Smith, R. C., 1989. A simple analytical formula to compute clear sky total and photosynthetically available solar irradiance at the ocean surface. Journal of Geophysical Research: Oceans 94 (C7), 9731-9742.

Gamon, J., Penuelas, J., Field, C., 1992. A narrow-waveband spectral index that tracks diurnal changes in photosynthetic efficiency. Remote Sensing of environment 41 (1), 35-44.

Garbulsky, M. F., Peñuelas, J., Gamon, J., Inoue, Y., Filella, I., 2011. The photochemical reflectance index $(\mathrm{PRI})$ and the remote sensing of leaf, 
canopy and ecosystem radiation use efficiencies: A review and meta-analysis. Remote Sensing of Environment 115 (2), 281-297.

Gastellu-Etchegorry, J.-P., Demarez, V., Pinel, V., Zagolski, F., 1996. Modeling radiative transfer in heterogeneous $3-\mathrm{D}$ vegetation canopies. Remote sensing of environment 58 (2), 131-156.

Gastellu-Etchegorry, J.-P., Lauret, N., Yin, T., Landier, L., Kallel, A. Malenovskỳ, Z., Al Bitar, A., Aval, J., Benhmida, S., Qi, J., et al., 2017. DART: Recent advances in remote sensing data modeling with atmosphere, polarization, and chlorophyll fluorescence. IEEE Journal of Selected Topics in Applied Earth Observations and Remote Sensing.

Genty, B., Briantais, J.-M., Baker, N. R., 1989. The relationship between the quantum yield of photosynthetic electron transport and quenching of chlorophyll fluorescence. Biochimica et Biophysica Acta (BBA)-General Subjects $990(1), 87-92$.

Gitelson, A. A., Gritz, Y., Merzlyak, M. N., 2003. Relationships between leaf chlorophyll content and spectral reflectance and algorithms for nondestructive chlorophyll assessment in higher plant leaves. Journal of Plant Physiology 160 (3), 271-282.

Gitelson, A. A., Keydan, G. P., Merzlyak, M. N., 2006. Three-band model for noninvasive estimation of chlorophyll, carotenoids, and anthocyanin contents in higher plant leaves. Geophysical Research Letters 33 (11).

Godfray, H. C. J., Beddington, J. R., Crute, I. R., Haddad, L., Lawrence, D., Muir, J. F., Pretty, J., Robinson, S., Thomas, S. M., Toulmin, C., 2010. Food security: the challenge of feeding 9 billion people. science 327 (5967), $812-818$

Goetz, S. J., Prince, S. D., Goward, S. N., Thawley, M. M., Small, J., 1999. Satellite remote sensing of primary production: an improved production efficiency modeling approach. Ecological Modelling 122 (3), 239-255. 
Goulden, M. L., Munger, J. W., FAN, S.-M., Daube, B. C., Wofsy, S. C., 1996. Measurements of carbon sequestration by long-term eddy covariance: Methods and a critical evaluation of accuracy. Global change biology 2 (3), 169-182.

Govaerts, Y. M., Verstraete, M. M., 1998. Raytran: A Monte Carlo ray-tracing model to compute light scattering in three-dimensional heterogeneous media. IEEE Transactions on geoscience and remote sensing 36 (2), 493505.

Grace, J., Nichol, C., Disney, M., Lewis, P., Quaife, T., Bowyer, P., 2007. Can we measure terrestrial photosynthesis from space directly, using spectral reflectance and fluorescence? Global Change Biology 13 (7), 1484-1497.

Guan, K., Berry, J. A., Zhang, Y., Joiner, J., Guanter, L., Badgley, G., Lobell, D. B., 2016. Improving the monitoring of crop productivity using spaceborne solar-induced fluorescence. Global change biology 22 (2), 716726.

Guanter, L., Aben, I., Tol, P., Krijger, J., Hollstein, A., Köhler, P., Damm, A., Joiner, J., Frankenberg, C., Landgraf, J., 2015. Potential of the TROPOspheric Monitoring Instrument (TROPOMI) onboard the Sentinel-5 Precursor for the monitoring of terrestrial chlorophyll fluorescence. Atmospheric Measurement Techniques 8 (3), 1337-1352.

Guanter, L., Alonso, L., Gómez-Chova, L., Meroni, M., Preusker, R., Fischer, J., Moreno, J., 2010. Developments for vegetation fluorescence retrieval from spaceborne high-resolution spectrometry in the o2-a and o2-b absorption bands. Journal of Geophysical Research: Atmospheres 115 (D19).

Guanter, L., Rossini, M., Colombo, R., Meroni, M., Frankenberg, C., Lee, J.-E., Joiner, J., 2013. Using field spectroscopy to assess the potential of statistical approaches for the retrieval of sun-induced chlorophyll fluorescence from ground and space. Remote Sensing of Environment 133, $52-61$. 
Guanter, L., Zhang, Y., Jung, M., Joiner, J., Voigt, M., Berry, J. A., Frankenberg, C., Huete, A. R., Zarco Tejada, P., Lee, J.-E., et al., 2014. Global and time-resolved monitoring of crop photosynthesis with chlorophyll fluorescence. Proceedings of the National Academy of Sciences 111 (14) E1327-E1333.

Guanter, L., Zhang, Y., Kohler, P., Walther, S., Frankenberg, C., Joiner, J., 2016. Overview of global monitoring of terrestrial chlorophyll fluorescence from space.

Hawkesford, M. J., Araus, J.-L., Park, R., Calderini, D., Miralles, D., Shen, T., Zhang, J., Parry, M. A., 2013. Prospects of doubling global wheat yields. Food and Energy Security 2 (1), 34-48.

He, L., Chen, J. M., Liu, J., Mo, G., Joiner, J., 2017. Angular normalization of GOME-2 sun-induced chlorophyll fluorescence observation as a better proxy of vegetation productivity. Geophysical Research Letters.

Heinsch, F. A., Zhao, M., Running, S. W., Kimball, J. S., Nemani, R. R., Davis, K. J., Bolstad, P. V., Cook, B. D., Desai, A. R., Ricciuto, D. M., et al., 2006. Evaluation of remote sensing based terrestrial productivity from MODIS using regional tower eddy flux network observations. IEEE Transactions on Geoscience and Remote Sensing 44 (7), 1908-1925.

Hernández-Clemente, R., North, P., Hornero, A., Zarco-Tejada, P., 2017. Assessing the effects of forest health on sun-induced chlorophyll fluorescence using the FluorFLIGHT 3-D radiative transfer model to account for forest structure. Remote Sensing of Environment 193, 165-179.

Houborg, R., Soegaard, H., Boegh, E., 2007. Combining vegetation index and model inversion methods for the extraction of key vegetation biophysical parameters using Terra and Aqua MODIS reflectance data. Remote Sensing of Environment 106 (1), 39-58.

Huang, D., Knyazikhin, Y., Dickinson, R. E., Rautiainen, M., Stenberg, P., Disney, M., Lewis, P., Cescatti, A., Tian, Y., Verhoef, W., et al., 2007. 
Canopy spectral invariants for remote sensing and model applications. Remote Sensing of Environment 106 (1), 106-122.

Idso, S., Kimball, B., Anderson, M., Mauney, J., 1987. Effects of atmospheric $\mathrm{CO} 2$ enrichment on plant growth: the interactive role of air temperature. Agriculture, ecosystems \& environment 20 (1), 1-10.

Jackson, R. D., Idso, S., Reginato, R., Pinter, P., 1981. Canopy temperature as a crop water stress indicator. Water Resources Research 17 (4), 11331138 .

Jacquemoud, S., 1993. Inversion of the PROSPECT + SAIL canopy reflectance model from AVIRIS equivalent spectra: theoretical study. Remote Sensing of Environment 44 (2-3), 281-292.

Jacquemoud, S., Baret, F., 1990. PROSPECT: A model of leaf optical properties spectra. Remote sensing of environment 34 (2), 75-91.

Jacquemoud, S., Baret, F., Andrieu, B., Danson, F., Jaggard, K., 1995. Extraction of vegetation biophysical parameters by inversion of the PROSPECT + SAIL models on sugar beet canopy reflectance data. Application to TM and AVIRIS sensors. Remote Sensing of Environment 52 (3), 163172 .

Jacquemoud, S., Verhoef, W., Baret, F., Bacour, C., Zarco Tejada, P. J., Asner, G. P., François, C., Ustin, S. L., 2009. PROSPECT+ SAIL models: A review of use for vegetation characterization. Remote Sensing of Environment 113, S56-S66.

Joiner, J., Guanter, L., Lindstrot, R., Voigt, M., Vasilkov, A., Middleton, E., Huemmrich, K., Yoshida, Y., Frankenberg, C., 2013. Global monitoring of terrestrial chlorophyll fluorescence from moderate spectral resolution nearinfrared satellite measurements: Methodology, simulations, and application to gome-2. Atmospheric Measurement Techniques 6 (2), 2803-2823. 
Joiner, J., Yoshida, Y., Vasilkov, A., Middleton, E., et al., 2011. First observations of global and seasonal terrestrial chlorophyll fluorescence from space. Biogeosciences 8 (3), 637-651.

Joiner, J., Yoshida, Y., Vasilkov, A., Schaefer, K., Jung, M., Guanter, L., Zhang, Y., Garrity, S., Middleton, E., Huemmrich, K., et al., 2014. The seasonal cycle of satellite chlorophyll fluorescence observations and its relationship to vegetation phenology and ecosystem atmosphere carbon exchange. Remote Sensing of Environment 152, 375-391.

Jolly, W. M., Dobbertin, M., Zimmermann, N. E., Reichstein, M., 2005. Divergent vegetation growth responses to the 2003 heat wave in the Swiss Alps. Geophysical Research Letters 32 (18).

Jung, M., Reichstein, M., Bondeau, A., 2009. Towards global empirical upscaling of fluxnet eddy covariance observations: validation of a model tree ensemble approach using a biosphere model. Biogeosciences 6 (10), 2001-2013.

Justice, C., Townshend, J., Vermote, E., Masuoka, E., Wolfe, R., Saleous, N., Roy, D., Morisette, J., 2002. An overview of MODIS Land data processing and product status. Remote sensing of Environment 83 (1-2), 3-15.

Justice, C. O., Vermote, E., Townshend, J. R., Defries, R., Roy, D. P., Hall, D. K., Salomonson, V. V., Privette, J. L., Riggs, G., Strahler, A., et al., 1998. The Moderate Resolution Imaging Spectroradiometer (MODIS): Land remote sensing for global change research. IEEE Transactions on Geoscience and Remote Sensing 36 (4), 1228-1249.

Kasahara, M., Kagawa, T., Oikawa, K., Suetsugu, N., Miyao, M., Wada, M., 2002. Chloroplast avoidance movement reduces photodamage in plants. Nature 420 (6917), 829.

Khosravi, N., Vountas, M., Rozanov, V. V., Bracher, A., Wolanin, A., Burrows, J. P., 2015. Retrieval of terrestrial plant fluorescence based on the in-filling of far-red fraunhofer lines using SCIAMACHY observations. Frontiers in Environmental Science 3, 78. 
Knipling, E. B., 1970. Physical and physiological basis for the reflectance of visible and near-infrared radiation from vegetation. Remote sensing of environment 1 (3), 155-159.

Knyazikhin, Y., Schull, M. A., Stenberg, P., Mõttus, M., Rautiainen, M., Yang, Y., Marshak, A., Carmona, P. L., Kaufmann, R. K., Lewis, P., et al., 2013. Hyperspectral remote sensing of foliar nitrogen content. Proceedings of the National Academy of Sciences 110 (3), E185-E192.

Koffi, E., Rayner, P., Norton, A., Frankenberg, C., Scholze, M., 2015. Investigating the usefulness of satellite-derived fluorescence data in inferring gross primary productivity within the carbon cycle data assimilation system. Biogeosciences 12 (13), 4067-4084.

Köhler, P., Guanter, L., Joiner, J., 2015. A linear method for the retrieval of sun-induced chlorophyll fluorescence from GOME-2 and SCIAMACHY data. Atmospheric Measurement Techniques 8 (6), 2589.

Köhler, P., Guanter, L., Kobayashi, H., Walther, S., Yang, W., 2018. Assessing the potential of sun-induced fluorescence and the canopy scattering coefficient to track large-scale vegetation dynamics in Amazon forests. Remote Sensing of Environment 204, 769 - 785.

Krause, G., Weis, E., 1991. Chlorophyll fluorescence and photosynthesis: the basics. Annual review of plant biology 42 (1), 313-349.

Kuusk, A., 2001. A two-layer canopy reflectance model. Journal of Quantitative Spectroscopy and Radiative Transfer 71 (1), 1-9.

Levitt, J., et al., 1980. Responses of Plants to Environmental Stress, Volume 1: Chilling, Freezing, and High Temperature Stresses. Academic Press.

Lieth, H., 1975. Modeling the primary productivity of the world. En: Primary productivity of the biosphere. Springer, pp. 237-263.

Liu, J., Skidmore, A. K., Jones, S., Wang, T., Heurich, M., Zhu, X., Shi, Y., 2018. Large off-nadir scan angle of airborne LiDAR can severely affect the 
estimates of forest structure metrics. ISPRS journal of photogrammetry and remote sensing 136, 13-25.

Liu, L., Liu, X., Wang, Z., Zhang, B., 2016. Measurement and analysis of bidirectional SIF emissions in wheat canopies. IEEE Transactions on Geoscience and Remote Sensing 54 (5), 2640-2651.

Liu, S., Peng, Y., Du, W., Le, Y., Li, L., 2015. Remote estimation of leaf and canopy water content in winter wheat with different vertical distribution of water-related properties. Remote Sensing 7 (4), 4626-4650.

Long, S., Farage, P., Garcia, R., 1996. Measurement of leaf and canopy photosynthetic CO2 exchange in the field. Journal of Experimental Botany 47 (11), 1629-1642.

Long, S., Humphries, S., Falkowski, P. G., 1994. Photoinhibition of photosynthesis in nature. Annual review of plant biology 45 (1), 633-662.

Los, S., Pollack, N., Parris, M., Collatz, G., Tucker, C., Sellers, P., Malmström, C., DeFries, R., Bounoua, L., Dazlich, D., 2000. A global 9-yr biophysical land surface dataset from noaa avhrr data. Journal of Hydrometeorology 1 (2), 183-199.

Maier, S. W., Günther, K. P., Stellmes, M., 2003. Sun-induced fluorescence: A new tool for precision farming. Digital imaging and spectral techniques: Applications to precision agriculture and crop physiology (digitalimaginga), 209-222.

Martens, S. N., Ustin, S. L., Rousseau, R. A., 1993. Estimation of tree canopy leaf area index by gap fraction analysis. Forest Ecology and Management 61 (1), 91-108.

Maxwell, K., Johnson, G. N., 2000. Chlorophyll fluorescencea practical guide. Journal of experimental botany 51 (345), 659-668.

McDonald, G., Paulsen, G., 1997. High temperature effects on photosynthesis and water relations of grain legumes. Plant and Soil 196 (1), 47-58. 
Meroni, M., Busetto, L., Colombo, R., Guanter, L., Moreno, J., Verhoef, W., 2010. Performance of spectral fitting methods for vegetation fluorescence quantification. Remote Sensing of Environment 114 (2), 363-374.

Meroni, M., Rossini, M., Guanter, L., Alonso, L., Rascher, U., Colombo, R., Moreno, J., 2009. Remote sensing of solar-induced chlorophyll fluorescence: Review of methods and applications. Remote Sensing of Environment 113 (10), 2037-2051.

Miao, G., Guan, K., Yang, X., Bernacchi, C. J., Berry, J. A., DeLucia, E. H., Wu, J., Moore, C. E., Meacham, K., Cai, Y., Peng, B., Kimm, H., Masters, M. D., 2018. Sun-induced chlorophyll fluorescence, photosynthesis, and light use efficiency of a soybean field. Journal of Geophysical Research: Biogeosciences.

Migliavacca, M., Perez-Priego, O., Rossini, M., El-Madany, T. S., Moreno, G., van der Tol, C., Rascher, U., Berninger, A., Bessenbacher, V., Burkart, A., et al., 2017. Plant functional traits and canopy structure control the relationship between photosynthetic CO2 uptake and far-red suninduced fluorescence in a Mediterranean grassland under different nutrient availability. New Phytologist.

Miller, J., Berger, M., Goulas, Y., Jacquemond, S., Mohammed, G., Moise, N., Moreno, J., Moya, I., Pedrós, R., 2005. Development of a vegetation fluorescence canopy model. ESA Scientific and Technical Publications Branch, ESTEC.

Monteith, J., 1972. Solar radiation and productivity in tropical ecosystems. Journal of applied ecology 9 (3), 747-766.

Mõttus, M., Stenberg, P., 2008. A simple parameterization of canopy reflectance using photon recollision probability. Remote Sensing of Environment 112 (4), 1545-1551.

Moya, I., Camenen, L., Evain, S., Goulas, Y., Cerovic, Z., Latouche, G., Flexas, J., Ounis, A., 2004. A new instrument for passive remote sensing: 1. 
measurements of sunlight-induced chlorophyll fluorescence. Remote Sensing of Environment 91 (2), 186-197.

Munro, R., Lang, R., Klaes, D., Poli, G., Retscher, C., Lindstrot, R., Huckle, R., Lacan, A., Grzegorski, M., Holdak, A., et al., 2016. The GOME-2 instrument on the Metop series of satellites: instrument design, calibration, and level 1 data processing-an overview. Atmospheric Measurement Techniques 9 (3), 1279-1301.

Myneni, R., Hoffman, S., Knyazikhin, Y., Privette, J., Glassy, J., Tian, Y., Wang, Y., Song, X., Zhang, Y., Smith, G., et al., 2002. Global products of vegetation leaf area and fraction absorbed PAR from year one of MODIS data. Remote sensing of environment 83 (1), 214-231.

Myneni, R. B., 1991. Modeling radiative transfer and photosynthesis in threedimensional vegetation canopies. Agricultural and Forest Meteorology 55 (3-4), 323-344.

Nakaji, T., Oguma, H., Fujinuma, Y., 2006. Seasonal changes in the relationship between photochemical reflectance index and photosynthetic light use efficiency of japanese larch needles. International Journal of Remote Sensing 27 (3), 493-509.

Nascimento, A. R. T., Fagg, J. M. F., Fagg, C. W., 2007. Canopy openness and LAI estimates in two seasonally deciduous forests on limestone outcrops in central brazil using hemispherical photographs. Revista Árvore 31 (1), 167-176.

Nichol, C. J., Huemmrich, K. F., Black, T. A., Jarvis, P. G., Walthall, C. L., Grace, J., Hall, F. G., 2000. Remote sensing of photosynthetic-light-use efficiency of boreal forest. Agricultural and Forest Meteorology 101 (2-3), $131-142$.

Nocedal, J., Wright, S., 2006. Numerical optimization. Springer Science \& Business Media. 
Paruelo, J. M., Epstein, H. E., Lauenroth, W. K., Burke, I. C., 1997. ANPP estimates from NDVI for the central grassland region of the United States. Ecology 78 (3), 953-958.

Pastenes, C., Horton, P., 1996. Effect of high temperature on photosynthesis in beans (I. Oxygen evolution and chlorophyll fluorescence). Plant Physiology $112(3), 1245-1251$.

Pearson, R. L., Miller, L. D., 1972. Remote spectral measurements as a method for determining plant cover. Technical report (US International Biological Program Grassland Biome); no 167.

Pedrós, R., Goulas, Y., Jacquemoud, S., Louis, J., Moya, I., 2010. Fluormodleaf: A new leaf fluorescence emission model based on the prospect model. Remote Sensing of Environment 114 (1), 155-167.

Pedrós, R., Moya, I., Goulas, Y., Jacquemoud, S., 2008. Chlorophyll fluorescence emission spectrum inside a leaf. Photochemical \& Photobiological Sciences 7 (4), 498-502.

Peñuelas, J., Filella, I., 1998. Visible and near-infrared reflectance techniques for diagnosing plant physiological status. Trends in plant science 3 (4), $151-156$.

Plascyk, J. A., 1975. The MK II Fraunhofer line discriminator (FLD-II) for airborne and orbital remote sensing of solar-stimulated luminescence. Optical Engineering 14 (4), 144339.

Porcar Castell, A., Tyystjärvi, E., Atherton, J., van der Tol, C., Flexas, J., Pfündel, E. E., Moreno, J., Frankenberg, C., Berry, J. A., 2014. Linking chlorophyll a fluorescence to photosynthesis for remote sensing applications: mechanisms and challenges. Journal of Experimental Botany, eru191.

Rahmstorf, S., Coumou, D., 2011. Increase of extreme events in a warming world. Proceedings of the National Academy of Sciences 108 (44), 1790517909. 
Rascher, U., Alonso, L., Burkart, A., Cilia, C., Cogliati, S., Colombo, R., Damm, A., Drusch, M., Guanter, L., Hanus, J., et al., 2015. Sun-induced fluorescence-a new probe of photosynthesis: First maps from the imaging spectrometer hyplant. Global change biology 21 (12), 4673-4684.

Rautiainen, M., Stenberg, P., 2005. Application of photon recollision probability in coniferous canopy reflectance simulations. Remote Sensing of Environment 96 (1), 98-107.

Reichstein, M., Ciais, P., Papale, D., Valentini, R., Running, S., Viovy, N., Cramer, W., Granier, A., Ogee, J., Allard, V., et al., 2007. Reduction of ecosystem productivity and respiration during the European summer 2003 climate anomaly: a joint flux tower, remote sensing and modelling analysis. Global Change Biology 13 (3), 634-651.

Reyer, C. P., Leuzinger, S., Rammig, A., Wolf, A., Bartholomeus, R. P., Bonfante, A., de Lorenzi, F., Dury, M., Gloning, P., Abou Jaoudé, R., et al., 2013. A plant's perspective of extremes: terrestrial plant responses to changing climatic variability. Global Change Biology 19 (1), 75-89.

Richter, R., Schlapfer, D., 2012. Atmospheric/topographic correction for airborne imagery: Atcor-4 user guide. DLR IB, 565-02.

Rosema, A., Snel, J., Zahn, H., Buurmeijer, W., Van Hove, L., 1998. The relation between laser-induced chlorophyll fluorescence and photosynthesis. Remote sensing of environment 65 (2), 143-154.

Rossini, M., Nedbal, L., Guanter, L., Ač, A., Alonso, L., Burkart, A., Cogliati, S., Colombo, R., Damm, A., Drusch, M., et al., 2015. Red and far red sun-induced chlorophyll fluorescence as a measure of plant photosynthesis. Geophysical Research Letters 42 (6), 1632-1639.

Rouse Jr, J., Haas, R., Schell, J., Deering, D., 1974. Monitoring vegetation systems in the Great Plains with ERTS. NASA special publication 351, 309. 
Running, S. W., Nemani, R. R., Heinsch, F. A., Zhao, M., Reeves, M., Hashimoto, H., 2004. A continuous satellite-derived measure of global terrestrial primary production. AIBS Bulletin 54 (6), 547-560.

Running, S. W., Thornton, P. E., Nemani, R., Glassy, J. M., 2000. Global terrestrial gross and net primary productivity from the Earth observing system. En: Methods in ecosystem science. Springer, pp. 44-57.

Running, S. W., Zhao, M., 2015. Daily GPP and annual NPP (MOD17a2/a3) products NASA Earth observing system MODIS land algorithm. MOD17 Users Guide.

Sage, R. F., Kubien, D. S., 2007. The temperature response of C3 and C4 photosynthesis. Plant, cell \& environment 30 (9), 1086-1106.

Schreiber, U., Schliwa, U., Bilger, W., 1986. Continuous recording of photochemical and non-photochemical chlorophyll fluorescence quenching with a new type of modulation fluorometer. Photosynthesis research 10 (1-2), $51-62$.

Schull, M., Ganguly, S., Samanta, A., Huang, D., Shabanov, N., Jenkins, J., Chiu, J. C., Marshak, A., Blair, J., Myneni, R., et al., 2007. Physical interpretation of the correlation between multi-angle spectral data and canopy height. Geophysical Research Letters 34 (18).

Smolander, S., Stenberg, P., 2005. Simple parameterizations of the radiation budget of uniform broadleaved and coniferous canopies. Remote Sensing of Environment 94 (3), 355-363.

Stenberg, P., Manninen, T., 2015. The effect of clumping on canopy scattering and its directional properties: a model simulation using spectral invariants. International Journal of Remote Sensing 36 (19-20), 5178-5191.

Stenberg, P., Mõttus, M., Rautiainen, M., 2016. Photon recollision probability in modelling the radiation regime of canopiesa review. Remote Sensing of Environment 183, 98-108. 
Sun, Y., Frankenberg, C., Wood, J. D., Schimel, D., Jung, M., Guanter, L., Drewry, D., Verma, M., Porcar-Castell, A., Griffis, T. J., et al., 2017. OCO-2 advances photosynthesis observation from space via solar-induced chlorophyll fluorescence. Science 358 (6360), eaam5747.

Sun, Y., Fu, R., Dickinson, R., Joiner, J., Frankenberg, C., Gu, L., Xia, Y., Fernando, N., 2015. Drought onset mechanisms revealed by satellite solarinduced chlorophyll fluorescence: Insights from two contrasting extreme events. Journal of Geophysical Research: Biogeosciences 120 (11), 24272440.

Turner, D. P., Ritts, W. D., Cohen, W. B., Gower, S. T., Zhao, M., Running, S. W., Wofsy, S. C., Urbanski, S., Dunn, A. L., Munger, J., 2003. Scaling gross primary production (GPP) over boreal and deciduous forest landscapes in support of MODIS GPP product validation. Remote Sensing of Environment 88 (3), 256-270.

Ustin, S. L., Gitelson, A. A., Jacquemoud, S., Schaepman, M., Asner, G. P., Gamon, J. A., Zarco-Tejada, P., 2009. Retrieval of foliar information about plant pigment systems from high resolution spectroscopy. Remote Sensing of Environment 113, S67-S77.

Valentinuz, O. R., Tollenaar, M., 2004. Vertical profile of leaf senescence during the grain-filling period in older and newer maize hybrids. Crop Science 44 (3), 827-834.

Van der Tol, C., Berry, J., Campbell, P., Rascher, U., 2014. Models of fluorescence and photosynthesis for interpreting measurements of solar-induced chlorophyll fluorescence. Journal of Geophysical Research: Biogeosciences 119 (12), 2312-2327.

Van der Tol, C., Rossini, M., Cogliati, S., Verhoef, W., Colombo, R., Rascher, U., Mohammed, G., 2016. A model and measurement comparison of diurnal cycles of sun-induced chlorophyll fluorescence of crops. Remote Sensing of Environment 186, 663-677. 
Van der Tol, C., Verhoef, W., Timmermans, J., Verhoef, A., Su, Z., 2009. An integrated model of soil-canopy spectral radiances, photosynthesis, fluorescence, temperature and energy balance. Biogeosciences 6 (12), 31093129 .

Van Wittenberghe, S., Alonso, L., Verrelst, J., Moreno, J., Samson, R., 2015. Bidirectional sun-induced chlorophyll fluorescence emission is influenced by leaf structure and light scattering propertiesA bottom-up approach. Remote Sensing of Environment 158, 169-179.

Van Wittenberghe, S., Alonso, L., Verrelst, J., Veroustraete, F., Valcke, R., Moreno, J., Samson, R., 2012. Estimating fluorescence emission of city trees in valencia: from leaf to canopy level. European Geosciences Union General Assembly.

Vasseur, D. A., DeLong, J. P., Gilbert, B., Greig, H. S., Harley, C. D., McCann, K. S., Savage, V., Tunney, T. D., O’Connor, M. I., 2014. Increased temperature variation poses a greater risk to species than climate warming. Proceedings of the Royal Society of London B: Biological Sciences 281 (1779), 20132612.

Verhoef, W., 1984. Light scattering by leaf layers with application to canopy reflectance modeling: the SAIL model. Remote sensing of environment $16(2), 125-141$.

Verhoef, W., 1985. Earth observation modeling based on layer scattering matrices. Remote sensing of environment 17 (2), 165-178.

Verhoef, W., 1998. Theory of radiative transfer models applied in optical remote sensing of vegetation canopies. sn].

Verhoef, W., Bach, H., 2007. Coupled soil-leaf-canopy and atmosphere radiative transfer modeling to simulate hyperspectral multi-angular surface reflectance and TOA radiance data. Remote Sensing of Environment 109 (2), 166-182. 
Verhoef, W., Bunnik, N., 1975. A Model Study on the Relations Between Crop Characteristics and Canopy Spectral Reflectance: 1. Deduction of Crop Parameters from Canopy Spectroreflectance Data: 2. Detectability of Variations in Crop Parameters by Multispectral Scanning. NIWARS.

Verhoef, W., Jia, L., Xiao, Q., Su, Z., 2007. Unified optical-thermal fourstream radiative transfer theory for homogeneous vegetation canopies. IEEE Transactions on Geoscience and Remote Sensing 45 (6), 1808-1822.

Verhoef, W., van der Tol, C., Middleton, E. M., 2018. Hyperspectral radiative transfer modeling to explore the combined retrieval of biophysical parameters and canopy fluorescence from FLEX-Sentinel-3 tandem mission multi-sensor data. Remote Sensing of Environment 204, 942-963.

Verrelst, J., Rivera, J. P., Leonenko, G., Alonso, L., Moreno, J., 2014. Optimizing LUT-based RTM inversion for semiautomatic mapping of crop biophysical parameters from Sentinel-2 and-3 data: Role of cost functions. IEEE Transactions on Geoscience and Remote Sensing 52 (1), 257-269.

Verrelst, J., Rivera, J. P., Van der Tol, C., Magnani, F., Mohammed, G., Moreno, J., 2015. Global sensitivity analysis of the SCOPE model: What drives simulated canopy-leaving sun-induced fluorescence? Remote Sensing of Environment 166, 8-21.

Verrelst, J., Van der Tol, C., Magnani, F., Sabater, N., Rivera, J. P., Mohammed, G., Moreno, J., 2016. Evaluating the predictive power of sun-induced chlorophyll fluorescence to estimate net photosynthesis of vegetation canopies: A SCOPE modeling study. Remote Sensing of Environment 176, 139-151.

Vilfan, N., Van der Tol, C., Muller, O., Rascher, U., Verhoef, W., 2016. Fluspect-B: A model for leaf fluorescence, reflectance and transmittance spectra. Remote Sensing of Environment 186, 596-615.

Vilfan, N., Van der Tol, C., Yang, P., Wyber, R., Malenovskỳ, Z., Robinson, S. A., Verhoef, W., 2018. Extending Fluspect to simulate xanthophyll 
driven leaf reflectance dynamics. Remote Sensing of Environment 211, $345-356$.

Viña, A., Gitelson, A. A., 2005. New developments in the remote estimation of the fraction of absorbed photosynthetically active radiation in crops. Geophysical Research Letters 32 (17).

Vogelmann, T., Han, T., 2000. Measurement of gradients of absorbed light in spinach leaves from chlorophyll fluorescence profiles. Plant, Cell \& Environment 23 (12), 1303-1311.

Wang, Q., Li, P., 2013. Canopy vertical heterogeneity plays a critical role in reflectance simulation. Agricultural and Forest Meteorology 169, 111-121.

Weiss, M., Baret, F., Smith, G., Jonckheere, I., Coppin, P., 2004. Review of methods for in situ leaf area index (LAI) determination: Part II. Estimation of LAI, errors and sampling. Agricultural and forest meteorology 121 (1-2), $37-53$.

White, M. A., Thornton, P. E., Running, S. W., Nemani, R. R., 2000. Parameterization and sensitivity analysis of the BIOME-BGC terrestrial ecosystem model: net primary production controls. Earth interactions $4(3), 1-85$.

Widlowski, J.-L., Taberner, M., Pinty, B., Bruniquel-Pinel, V., Disney, M., Fernandes, R., Gastellu-Etchegorry, J.-P., Gobron, N., Kuusk, A., Lavergne, T., et al., 2007. Third Radiation Transfer Model Intercomparison (RAMI) exercise: Documenting progress in canopy reflectance models. Journal of Geophysical Research: Atmospheres 112 (D9).

Wieneke, S., Ahrends, H., Damm, A., Pinto, F., Stadler, A., Rossini, M., Rascher, U., 2016. Airborne based spectroscopy of red and far-red suninduced chlorophyll fluorescence: Implications for improved estimates of gross primary productivity. Remote Sensing of Environment 184, 654-667. 
Wullschleger, S. D., 1993. Biochemical limitations to carbon assimilation in c3 plantsa retrospective analysis of the $\mathrm{A} / \mathrm{Ci}$ curves from 109 species. Journal of Experimental Botany 44 (5), 907-920.

Xiao, J., Chen, J., Davis, K. J., Reichstein, M., 2012. Advances in upscaling of eddy covariance measurements of carbon and water fluxes. Journal of Geophysical Research: Biogeosciences 117 (G1).

Xiao, J., Zhuang, Q., Baldocchi, D. D., Law, B. E., Richardson, A. D., Chen, J., Oren, R., Starr, G., Noormets, A., Ma, S., et al., 2008. Estimation of net ecosystem carbon exchange for the conterminous united states by combining modis and ameriflux data. Agricultural and Forest Meteorology 148 (11), 1827-1847.

Xu, X., Wang, J., Wang, Y., Kokhanovsky, A., 2018. Passive remote sensing of aerosol height. En: Remote Sensing of Aerosols, Clouds, and Precipitation. Elsevier, pp. 1-22.

Yamori, W., Hikosaka, K., Way, D. A., 2014. Temperature response of photosynthesis in $\mathrm{C} 3, \mathrm{C} 4$, and CAM plants: temperature acclimation and temperature adaptation. Photosynthesis Research 119 (1-2), 101-117.

Yang, P., van der Tol, C., 2018. Linking canopy scattering of far-red suninduced chlorophyll fluorescence with reflectance. Remote Sensing of Environment 209, 456-467.

Yang, P., Verhoef, W., van der Tol, C., 2017. The mSCOPE model: A simple adaptation to the SCOPE model to describe reflectance, fluorescence and photosynthesis of vertically heterogeneous canopies. Remote Sensing of Environment 201, 1-11.

Yang, X., Tang, J., Mustard, J. F., Lee, J.-E., Rossini, M., Joiner, J., Munger, J. W., Kornfeld, A., Richardson, A. D., 2015. Solar-induced chlorophyll fluorescence that correlates with canopy photosynthesis on diurnal and seasonal scales in a temperate deciduous forest. Geophysical Research Letters 42 (8), 2977-2987. 
Zarco-Tejada, P., Pushnik, J., Dobrowski, S., Ustin, S., 2003. Steady-state chlorophyll a fluorescence detection from canopy derivative reflectance and double-peak red-edge effects. Remote Sensing of Environment 84 (2), 283-294.

Zarco-Tejada, P. J., Berni, J. A., Suárez, L., Sepulcre-Cantó, G., Morales, F., Miller, J., 2009. Imaging chlorophyll fluorescence with an airborne narrow-band multispectral camera for vegetation stress detection. Remote Sensing of Environment 113 (6), 1262-1275.

Zarco-Tejada, P. J., González-Dugo, V., Berni, J. A., 2012. Fluorescence, temperature and narrow-band indices acquired from a UAV platform for water stress detection using a micro-hyperspectral imager and a thermal camera. Remote Sensing of Environment 117, 322-337.

Zarco-Tejada, P. J., Miller, J. R., Pedrós, R., Verhoef, W., Berger, M., 2006. Fluormodgui v3. 0: A graphic user interface for the spectral simulation of leaf and canopy chlorophyll fluorescence. Computers \& Geosciences 32 (5), $577-591$.

Zhang, Y., Guanter, L., Berry, J. A., Joiner, J., Tol, C., Huete, A., Gitelson, A., Voigt, M., Köhler, P., 2014. Estimation of vegetation photosynthetic capacity from space-based measurements of chlorophyll fluorescence for terrestrial biosphere models. Global Change Biology 20 (12), 3727-3742.

Zhao, C., Li, H., Li, P., Yang, G., Gu, X., Lan, Y., 2017. Effect of vertical distribution of crop structure and biochemical parameters of winter wheat on canopy reflectance characteristics and spectral indices. IEEE Transactions on Geoscience and Remote Sensing 55 (1), 236-247.

Zhao, F., Dai, X., Verhoef, W., Guo, Y., van der Tol, C., Li, Y., Huang, Y., 2016. FluorWPS: A Monte Carlo ray-tracing model to compute sun-induced chlorophyll fluorescence of three-dimensional canopy. Remote Sensing of Environment 187, 385-399. 


\section{Bibliography}

Zygielbaum, A. I., Gitelson, A. A., Arkebauer, T. J., Rundquist, D. C., 2009. Non-destructive detection of water stress and estimation of relative water content in maize. Geophysical Research Letters 36 (12). 


\section{Summary}

Plants are a dynamic part of our planet. They have made the world habitable and formed the climate, through a fundamental process, photosynthesis. Photosynthesis absorbs energy from the sun, removes $\mathrm{CO}_{2}$ from the atmosphere and records it, and creates $\mathrm{O}_{2}$ for life on Earth. Tracking the speed of photosynthesis via Earth observation is needed to better understand the interactions of different processes in the environment, and it is essential from the point of view of food safety. It is not feasible to obtain a global and continuous time series of photosynthesis by measuring the exchange of gases alone. Earth observation with satellites offers a possible outcome, but measuring reflection with satellites offers no more than a rough estimate of photosynthesis. The sunlight-induced fluorescence of chlorophyll in plants (SIF) is a promising candidate to fill this gap. Measurements of SIF contain information about both the collection of sunlight, the energy source of photosynthesis, and the efficiency with which the captured light is used for capturing carbon. During the past decades SIF has been used as a direct measure for primary production (GPP), but also as an imposed restriction on models for GPP. The main challenges in the use of SIF for photosynthesis are: 1) obtaining quantitative information on parameters related to photosynthesis from measurements of SIF above the crop via model inversion, and 2) quantifying the mechanisms by which SIF and photosynthesis are linked to each other at the sub-cellular level of photo systems. This dissertation examines the first problem, and aims to deepen the understanding of SIF as measured above the vegetation. The three processes that are responsible 
for SIF are quantified in this study, namely the absorption of sunlight, the emission of fluorescence, and the (re) absorption of fluorescence. By separately quantifying these three processes, SIF can be corrected for the (re) absorption in the vegetation. The resulting signal is a better measure for photosynthesis than the uncorrected SIF. Further normalization through the light absorption provides information about the efficiency of the emission: a measure for the distribution of the energy in the photo systems over various processes, including photochemistry. The study uses models for radiation transport (RTMs) for the interpretation of simultaneous measurements of SIF and reflection above the vegetation. The approach proved to be well suited for evaluating the effects of the absorption of sunlight and fluorescence. It turned out to be possible to trace the functional response of various agricultural crops to a heat wave from measurements with the HyPlant sensor on an airplane. After analysis of the radiation transport, it was possible to estimate the scattering and reabsorption of the fluorescence from the reflection by means of a simple comparison. This simple comparison makes it possible to calculate the total produced fluorescence in the vegetation from the measured SIF and reflection. A new model for fPAR has emerged from a further analysis of the absorption of sunlight (fPAR). This model for fPAR, combined with the equation for total fluorescence, resulted in a very simple reflection index, FCVI, for both the absorption of sunlight and the scattering of fluorescence. FCVI is the difference between the near infrared and the panchromatic visible reflection. With this index, measurements of SIF with the satellite GOME-2 were subsequently corrected, resulting in global time series of the efficiency of the emission of fluorescence. The final part of this thesis describes a general radiation model for vegetation with a complex composition, modeled in the mSCOPE model. This model simulates the interaction of light and the energy balance in vegetation consisting of several layers. This model will also contribute to the interpretation of SIF measurements. This thesis contains a number of suitable approaches to interpret SIF measurements and to use them well. The measured SIF can be converted into an estimate of the total production of fluorescence in the 
vegetation and the efficiency of that production. This makes it possible to go beyond the empirical correlation between SIF and photosynthesis that until recently was common. Future studies should show how fluorescence depends on photosynthesis at the level of photo systems. 



\section{Samenvatting}

Planten vormen een dynamisch onderdeel van onze planeet. Zij hebben de wereld bewoonbaar gemaakt en het klimaat gevormd, door middel van een fundamenteel proces, fotosynthese. Door fotosynthese wordt energie van de zon opgevangen, $\mathrm{CO}_{2}$ uit de atmosfeer gehaald en vastgelegd, en $\mathrm{O}_{2}$ gevormd voor het leven op Aarde. Het bijhouden van de snelheid van fotosynthese via aardobservatie is nodig voor beter begrip van de wisselwerkingen van verschillende processen in het milieu, en het is essentieel uit het oogpunt van voedselveiligheid. Het is ondoenlijk om een mondiale en ononderbroken tijdserie van fotosynthese te verkrijgen door alleen het meten van de uitwisseling van gassen. Aardobservatie met satellieten biedt een mogelijke uitkomst, maar het meten van reflectie met satellieten biedt niet meer dan een ruwe schatting van de fotosynthese. De door zonlicht opgewekte fluorescentie van chlorofyl in planten (SIF) is een veelbelovende kandidaat om dit hiaat op te vullen. Metingen van SIF bevatten informatie over zowel het opvangen van zonlicht, de energiebron van fotosynthese, als de efficintie waarmee het ingevangen licht gebruikt wordt voor het vastleggen van koolstof. Gedurende de afgelopen decennia is SIF gebruikt als een directe maat voor de primaire productie (GPP), maar ook als een opgelegde beperking aan modellen voor GPP. De grootste uitdagingen bij het gebruik van SIF voor fotosynthese zijn: 1) het verkrijgen van kwantitatieve informatie over parameters gerelateerd aan de fotosynthese uit metingen van SIF boven het gewas via modelinversie, en 2) het kwantificeren de mechanismes waarmee SIF en fotosynthese aan elkaar gekoppeld zijn op het sub-cellulaire niveau 
van fotosystemen. Dit proefschrift gaat in op het eerste probleem, en beoogt het begrip van SIF als gemeten boven de vegetatie te verdiepen. De drie processen die verantwoordelijk zijn voor SIF zijn in deze studie gekwantificeerd, namelijk de absorptie van zonlicht, de emissie van fluorescentie, en de (her)absorptie van fluorescentie. Door het afzonderlijk kwantificeren van die drie processen, kan SIF worden gecorrigeerd voor de (her)absorptie in de vegetatie. Het resulterende signaal is een betere maat voor de fotosynthese dan de ongecorrigeerde SIF. Verdere normalisatie door de lichtabsorptie levert informatie over efficintie van de emissie: een maat voor de verdeling van het de energie in de fotosystemen over verschillende processen waaronder de photochemie. De studie maakt gebruik van modellen voor stralingstransport (RTMs) voor de interpretatie van gelijktijdige metingen van SIF en reflectie boven de vegetatie. De benadering bleek goed geschikt voor het evalueren van de effecten van de absorptie van zonlicht en fluorescentie. Het bleek mogelijk om de functionele reactie van verschillende landbouwgewassen op een hittegolf te achterhalen uit metingen met de HyPlant sensor op een vliegtuig. $\mathrm{Na}$ analyse van het stralingstransport bleek het goed mogelijk om de verstrooiing en herabsorptie van de fluorescentie te schatten uit de reflectie door middel van een eenvoudige vergelijking. Deze eenvoudige vergelijking maakt het mogelijk om de totale geproduceerde fluorescentie in de vegetatie te berekenen uit de gemeten SIF en reflectie. Uit een verdere analyse van de absorptie van zonlicht (fPAR) is een nieuw model voor fPAR voortgekomen. Dit model voor fPAR, gecombineerd met de vergelijking voor de totale fluorescentie, resulteerde in een zeer eenvoudige reflectieindex, FCVI, voor zowel de absorptie van zonlicht en de verstrooiing van fluorescentie. FCVI is het verschil tussen de nabij infrarode en de panchromatische zichtbare reflectie. Met deze index zijn vervolgens metingen van SIF met de satelliet GOME-2 gecorrigeerd, en dit resulteerde in mondiale tijdseries van de efficintie van de emissie van fluorescentie. Het laatste deel van dit proefschrift beschrijft een algemeen stralingsmodel voor vegetatie met een complexe samenstelling, vormgegeven in het model mSCOPE. Dit model simuleert de interactie van het licht en de energiebalans in vegetatie die uit meerdere lagen bestaat. 
Ook dit model zal bijdragen aan de interpretatie van metingen van SIF. Dit proefschrift bevat een aantal geschikte benaderingen om SIF metingen te interpreteren en goed te gebruiken. De gemeten SIF kan worden omgezet in een schatting van de totale productie van fluorescentie in het de vegetatie en de efficintie van die productie. Dit maakt het mogelijk om verder te gaan dan de empirische correlatie tussen SIF en fotosynthese die tot voor kort gebruikelijk was. Toekomstige studies moeten uitwijzen hoe fluorescentie afhangt van fotosynthese op het niveau van fotosystemen. 



\section{Author's biography and publications}

Peiqi Yang was born in December 1989 in Jiangxi Province, China. He received the B.Eng. degree on Geodesy and Geomatics Engineering from Wuhan University in 2011 and then he got the M.Sc. on Cartology and Geographic Information System in Beijing Normal University in 2011. In September 2011, he was awarded a four-year scholarship from China Scholarship Council (CSC) for perusing his Ph.D. degree at the Faculty of Geo-Information Science and Earth Observation (ITC), University of Twente. His research interests cover radiative transfer modeling, hyperspectral remote sensing of vegetation, chlorophyll fluorescence, and climate-vegetation interactions. 
Yang, P., van der Tol, C., 2018. Linking canopy scattering of far-red sun-induced chlorophyll fluorescence with reflectance. Remote Sensing of Environment 209, 456 - 467.

Yang, P., Verhoef, W., van der Tol, C., 2017. The mSCOPE model: A simple adaptation to the SCOPE model to describe reflectance, fluorescence and photosynthesis of vertically heterogeneous canopies. Remote Sensing of Environment 201, 1 - 11.

Yang, P., van der Tol, C., Verhoef, W., Damm, A., Schickling, A.,Kraska, T.,Muller, O.,Rascher, U., 2018. Response of Crops to a Heat Wave: Insights from Airborne based Reflectance and Chlorophyll Fluorescence Measurements. Remote Sensing of Environment, under review.

Yang, P., van der Tol, C., Verhoef, W., 2018. FCVI: A vegetation reflectance index for interpreting sun-induced chlorophyll fluorescence. Remote Sensing of Environment, under review.

Yang, P., van der Tol, C., A spectral invariant approach to modelling radiative transfer of sun-induced chlorophyll fluorescence. IEEE Geoscience and Remote Sensing Symposium (IGARSS), 2018.

Vilfan, N., van der Tol, C., Yang, P., et al., 2018. Extending Fluspect to simulate xanthophyll driven leaf reflectance dynamics. Remote Sensing of Environment 211, 345 - 356.

Celesti, M., van der Tol, C., Cogliati, S., Panigada, C., Yang, P., Pinto, F., Rascher, U., Miglietta, F., Colombo, R., Rossini, M., 2018. Exploring the physiological information of Sun-induced chlorophyll fluorescence through radiative transfer model inversion. Remote Sensing of Environment, 215, 97-108.

Cendrero-Mateo, M., van der Tol, C., Sakowska, K., Celesti, M., Schickling, A., Alberti, G., Alonso, L., Burkart, A., Cogliati, S., Colombo, R., Vedove, G., Julitta, T., Juszczak, R., Moreno, J., Muller, O., Panigada, C., Peressotti, A., Rademske, P., Rossini, M., Tagliabue, G., Vilfan, N., Yang, P., Miglietta, F., Rascher, U., Correcting for Sun Induced Fluorescence reabsorption - from leaf to canopy - in chlorophyll-deficient and green soybean plants. Remote Sensing of Environment, under review. 
Yang, P., Liu, Z. Remote sensing of solar-induced chlorophyll fluorescence from an unmanned airship platform. IEEE Geoscience and Remote Sensing Symposium (IGARSS), 2013.

Yang, P., Liu, Z., Ni, Z., Wang, R., and Wang, Q. (2013). Remote sensing of chlorophyll fluorescence at airborne level based on unmanned airship platform and hyperspectral sensor. Spectroscopy and Spectral Analysis, 33(11), 3101-3105.

Wang, R, Liu, Z., Feng, H., Yang, P., Wang, Q., Ni, Z., (2013). Extraction and analysis of solar-induced chlorophyll fluorescence of wheat with ground-based hyperspectral imaging system. Spectroscopy and Spectral Analysis, 33(9), 2451-2454.

Wang, R., Liu., Z, Yang, P., (2012) Principle and Progress in Remote Sensing of Vegetation Solar-induced Chlorophyll Fluorescence. Advances in Earth Sciences, 27(11). 



\section{ITC dissertations}

A complete list of ITC dissertations is online on the ITC website: www.itc.nl/research/phd/phd_graduates.aspx. 